\title{
PROCEDIMENTOS NUMÉRICOS E ESTIMAÇÃO DE COMPONENTES DE COVARIÂNCIA EM ANALISE MULTIVARIADA PELO MÉTODO DA MAXIMA VEROSSIMILHANÇA RESTRITA - MODELOS MISTOS APLICADOS AO MELHORAMENTO ANIMAL
}

RUI DA SILVA VERNEQUE

Zootecnista

Orientador: Prof. Dr. CASSIO ROBERTO DE MELO GODOI

\begin{abstract}
Tese apresentada à Escola Superior de Agricultura "Luiz de Queiroz", da Universidade de São Paulo, para obtenção do titulo de Doutor em Agronomia, Área de Concentração: Estatística e Experimentação Agronômica.
\end{abstract}

P I R A C I C A B A

Estado de São Paulo - Brasil

Outubro - 1994 
Ficha catalográfica preparada pela seção de Livros da Divisão de Biblioteca e Documentação - PCLQ/USP

Verneque, Rui da Silva

V531p Procedimentos numéricos e estimação de componentes de covariância em análise multivariada pelo método da máxima verossimilhança restrita - modelos mistos aplicados ao melhoramento animal. Piracicaba, 1994.

$157 \mathrm{p}$.

Tese - ESALQ

Bibliografia.

1. Análise de covariancia 2. Análise multivariada 3. Bovino-Melhoramento - Modelo matemático 4. Estatística aplicada 5. Método da máxima verossimilhança restrita 6. Modelo matemático I. Escola Superior de Agricultura Luiz de Queiroz, Piracicaba

CDD $\quad 636.2082$

519.535 
PROCEDIMENTOS NUMÉRICOS E ESTIMAÇÃO DE COMPONENTES DE COVARIÂNCIA EM ANÁLISE MULTIVARIADA PELO MÉTODO DA MÁXIMA VEROSSIMILHANÇA RESTRI'TA - MODELOS MIS'TOS APLICADOS AO MELHORAMENTO ANIMAL

Aprovada em: 12/12/94

Comissão julgadora:

Prof. Dr. Cássio Roberto de Melo Godoi ESALQ/USP

Prof. Dr. Décio Barbin ESALQ/USP

Prof. Dr. Irineu Umberto Packer ESALQ/USP

Prof. Dr. José Bento Sterman Ferraz

FZEA/USP

Dr. José Valente

rof. Dr. Cássio Roberto/de Melo Godoi Orientador 


\section{DEDICAÇÃO}

A meus pais, com gratidão,

A Dona Joana (in memorian), com saudades,

Aos meus irmãos, com lembranças,

A Iemar, Filipe e Débora, sentido do que faço,

dedico com carinho e amor. 


\section{AGRADECIMENTOS}

Ao Professor Dr. Cássio Roberto de Melo Godoi, pela orientação, confiança e estímulo.

Aos Professores Doutores Décio Barbin, Irineu Umberto Packer, José Bento Sterman Ferraz e Elias Nunes Martins e aos Pesquisadores Dr. José Valente e Mário Luiz Martinez, pelas sugestões e amizade.

A Professora Dr ${ }^{a}$ Clarice Garcia Borges Demétrio, "nossa adotada tia", pela consideração, ensinamentos e saudável convivência.

Aos Professores do Departamento de Matemática e Estatrstica da ESALQ / USP, pelos ensinamentos e convívio.

Aos Pesquisadores Dr. Ignacy Misztal, Dr ${ }^{\mathbf{a}}$, Karin Mayer e Dr. Keith G. Boldman e colaboradores, pela concessão dos programas para uso.

Ao Professor Dr. L. D. Van Vleck, pela disponibilidade, espirito de cientista e brilhantes sugestões.

Ao Dr. Normal R. Brockington, pelas sugestões e auxilio na elaboração do summary.

Aos colegas de curso Cesar, Gener, Joel, Rosana e Tadeu, pelo companheirismo e amizade.

A Luciane, uma pessoa especial, pela satisfação em servir bem.

Às secretárias do DME/ESALQ/USP nas pessoas da Ana, Rosa e Solange, por terem nos suportado.

Ao colega de trabalho e amigo, Newton Luis de Almeida, pela revisão lingürstica deste trabalho.

Ao Jorge A. Wiendl, pela ajuda no uso do processador de texto LAT $_{\mathrm{E}} \mathrm{X}$

Aos grandes amigos, Ildeu, Paulo Cecon e familiares, Nelson Donizete, Maria Cezira e famflia, pelo apoio, sinceridade e confiança.

A Empresa Brasileira de Pesquisa Agropecuária (EMBRAPA), em particular ao Centro Nacional de Pesquisa de Gado de Leite (CNPGL), pela oportunidade em realizar o curso.

Ao Conselho Nacional de Desenvolvimento Cientifico e Tecnológico (CNPq), pela bolsa concedida.

A todos que, direta ou indiretamente, colaboraram com esta importante etapa de minha vida. 
1 INTRODUÇÃO

2 REVISÃO DE LITERATURA

2.1 Procedimentos Numéricos para Resolução de Sistemas de Equações Lineares 6

2.1.1 Métodos Baseados na Triangulação . . . . . . . . . . . . . . 6

2.1.1.1 Sistema Triangular ................ 8

2.1.1.2 Eliminação de Gauss ou Condensação Pivotal . . . . . . . . 8

2.1.1.3 Métodos compactos ................... 10

2.1.1.3.1 Método Crout (ou Cholesky Geral) . . . . . . 10

2.1.1.3.2 Decomposição QR . . . . . . . . . . 12

2.1.1.3.3 Decomposição de Cholesky . . . . . . . . . . 12

2.1.2 Métodos baseados na diagonalização . . . . . . . . . . . . . 14

2.1.2.1 Eliminação de Gauss - Jordan . . . . . . . . . . . . . 14

2.1.2.2 Decomposição por Valores Singulares (DVS) . . . . . . 15

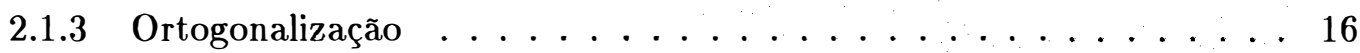

2.1.3.1 Método da Ortogonalização Matricial - Método de Gram-

Schmidt ..................... 16

2.1 .4 Métodos da Partição . . . . . . . . . . . . . . . 16

2.1.4.1 Procedimento Padrão de Partição . . . . . . . . . . 16

2.1 .5 Métodos Iterativos . . . . . . . . . . . . . . 18

2.1.5.1 Iteração Não Linear . . . . . . . . . . . . . . . . . . . . . . . . . 19

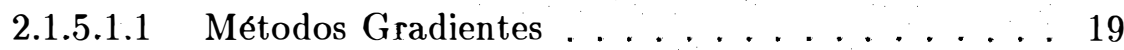

2.1.5.1.2 Método Simplex .............. 19

2.1.5.1.3 Método das Direções Conjugadas e Método de

Powell . . . . . . . . . . . . 22

2.1.5.2 Iteração Linear . . . . . . . . . . . . . 23

2.1.5.2.1 Método de Jacobi: iterações por deslocamentos simultaneos . . . . . . . . . . . 23

2.1.5.2.2 Método de Gauss-Seidel por deslocamentos sucessivos ..................... 24

2.1.5.2.3 Método de Gauss-Seidel para "trás" e para "frente" 25

2.1.5.2.4 Método SOR "Successive overrelaxation" . . . . 25

2.2 Procedimento Para Aceleração da Convergência: Método $\Delta^{2}$ de Aitken . . . 26

2.3 Métodos de Estimação de Componentes de Covariancia . . . . . . . . . 27

2.3.1 Métodos de Henderson . . . . . . . . . . . . . . 28

2.3.1.1 Método I de Henderson ............... 28

2.3.1.2 Método II de Henderson . . . . . . . . . . . . . 30

2.3.1.3 Método III de Henderson . . . . . . . . . . . . 33

2.3.1.4 Método IV de Henderson ................. 35

2.3 .2 Os Métodos MINQUE e MIVQUE .............. 36 
2.3.3 Método da Máxima Verossimilhança - ML . . . . . . . . . . . 40 2.3.3.1 Derivação dos componentes de variancia pelo método ML . 41

2.3.4 Método da Máxima Verossimilhança Restrita - REML . . . . . . . 43 2.3.4.1 Derivação dos componentes de variancia pelo método REML 44 2.3.4.1.1 REML a partir das equações MIVQUE . . . . . 44 2.3.4.1.2 REML a partir das equações de verossimilhança . 45 2.3.4.1.3 REML a partir da função de verossimilhança . . . 46

2.3.5 Método da Falsa Esperança . . . . . . . . . . . . . . . 52

2.3.6 Método da Verossimilhança Integrada - VEIL . . . . . . . . . 53

2.4 Algorítmo para Simulação de Modelos Mistos . . . . . . . . . . . . 58

2.4.1 Simulação de valores genéticos para características múltiplas sem parentesco entre animais . . . . . . . . . . . . 60

2.4.2 Simulação de valores genéticos para características múltiplas com parentesco entre animais . . . . . . . . . . . . 6 60

2.4.3 Simulação de valores genéticos e efeitos correlacionados para características múltiplas com parentesco entre animais . . . . . . . 61

3 MATERIAL E MÉTODOS

3.1 Descrição dos Sistemas DFREML, MTC e MTDFREML . . . . . . . . . 62

3.1.1 SISTEMA DFREML (Derivative Free Restricted Maximum Likeli-

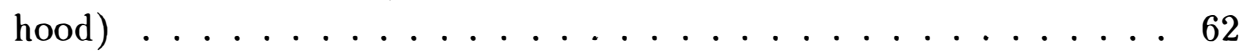

3.1.2 SISTEMA MTC ("Multitrait REML Estimation") . . . . . . . . . 65

3.1.3 SISTEMA MTDFREML (Multiple Trait Derivative Free Restricted

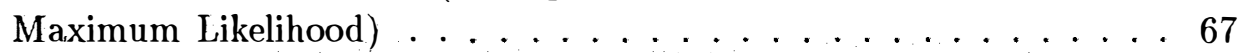

3.1 .4 Critérios de Convergência . . . . . . . . . . . 68

3.2 Dados Utilizados no Estudo . . . . . . . . . . . . . . . . . 69

3.2 .1 Dados Simulados . . . . . . . . . . . . . . . . . . . . . . . . . .

3.2 .2 Dados Reais . . . . . . . . . . . . . . . 76

3.3 Modelo Linear Misto Multivariado . . . . . . . . . . . . . . 77

3.4 Derivação das equações do modelo misto (MME) . . . . . . . 80

3.5 Estimadores dos Componentes de Covariancia . . . . . . . . . . . 83

3.5.1 Algorıtmo EM (Maximização de Esperanças) . . . . . . . . . . . 83

3.5 .2 Método Livre de Derivadas . . . . . . . . . . . . 87

3.6 Estimação dos Parametros Genéticos, Fenotf́picos e de meio ambiente . . . 93

4 RESULTADOS E DISCUSSÃO 95

4.1 Análise Univariada . . . . . . . . . . . . . . . . . 95

4.2 Análise Multivariada . . . . . . . . . . . . . . 102

5 CONCLUSÕES $\quad 147$

REFERÊNCIAS BIBLIOGRÁFICAS $\quad 149$

$\begin{array}{lr}\text { APENDICE } & 156\end{array}$ 


\section{LISTA DE TABELAS}

Tabela 1. Programas do sistema DFREML versão $2.1 .09 \ldots \ldots \ldots \ldots \ldots \ldots \ldots \ldots 6 . \ldots 6$

Tabela 2. Resumo das opções dos programas DFREML V. 2.1.09 ...........64

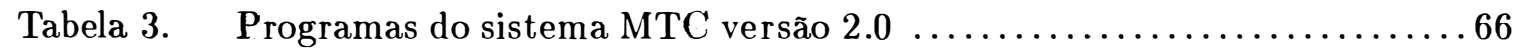

Tabela 4. Programas do sistema MTDREML . . . . . . . . . . . . . . . . . 67

Tabela 5. Características dos sistemas DFREML, MTC e MTDFREML ......668

Tabela 6. Matrizes de covariancias utilizadas para simulação dos dados para modelo animal sem efeito permanente de meio .............. 74

Tabela 6a. Matrizes de covariâncias utilizadas para simulação de modelos animal com efeito permanente de meio . . . . . . . . . . . . . 75

Tabela 6b. Herdabilidade $\left(h^{2}\right)$, repetibilidade $(r)$ e correlações genéticas $\left(r_{g}\right)$ e fenotípicas $\left(r_{p}\right)$ para os dados das tabelas 6 e 6 a $\ldots \ldots \ldots \ldots \ldots \ldots . \ldots 76$

Tabela 6c. Descrição dos arquivos de dados utilizados nas análises $\ldots \ldots \ldots \ldots \ldots 77$

Tabela 7. Modelos de análises para o sistema DFREML . . . . . . . . . . . . . 79

Tabela 7a. Modelos de análise para o sistema MTC $\ldots \ldots \ldots \ldots \ldots \ldots \ldots \ldots \ldots$

Tabela $7 \mathrm{~b}$. Modelos de análise para o sistema MTDFREML $\ldots \ldots \ldots \ldots \ldots \ldots \ldots$

Tabela 8. Estimativas dos componentes de variancia para os dados do arquivo UNIVA282.ARQ, Análise univariada - modelo animal ............ 98

Tabela 9. Estimativas dos componentes de variância e do coeficiente de herdabilidade para os dados do arquivo SIMUL050.MIS. Análise univaria-

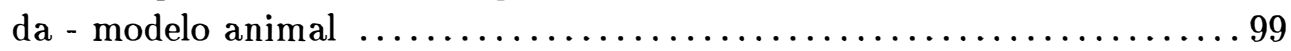

Tabela 10. Estimativas dos componentes de variancia, herdabilidade e repetibilidade, para os dados do arquivo CNPGL001.ARQ. Análise univa-

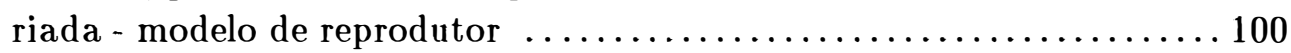

Tabela 10a. Estimativas dos componentes de variancia, herdabilidade e repetibilidade, para os dados do arquivo CNPGL001.ARQ. Análise univariada da Produção de leite no $1^{\circ}\left(P_{1}\right), 2^{\circ}\left(P_{2}\right)$ e $3^{\circ}\left(P_{3}\right)$ partos modelo animal .............................................. 101

Tabela 10b. Informações adicionais para os dados da Tabela $10 \mathrm{a} \ldots \ldots \ldots \ldots \ldots \ldots 1$

Tabela 10c. Estimativas dos componentes de variancia, herdabilidade, $\hat{c}^{2}$ e repetibilidade, para os dados do arquivo CNPGL001.ARQ. Análise

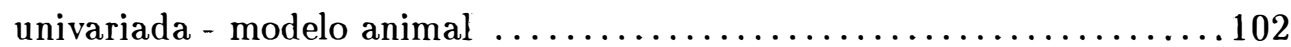

Tabela 11. Estimativas dos componentes de variancia, herdabilidade e correl acões genéticas, fenotípicas e de meio ambiente para os dados do ar-

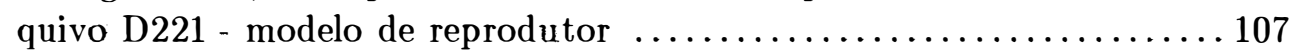

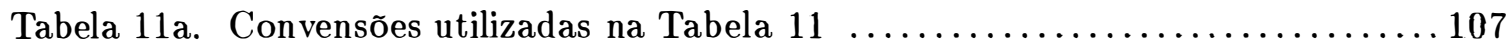

Tabela 12. Estimativas dos componentes de covariancia para dados do arquivo

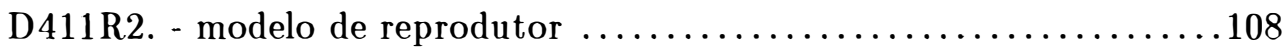

Tabela 13. Informações gerais referentes às análises dos dados do arquivo

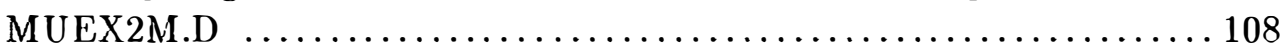

Tabela 13a. Valores iniciais utilizados no processo de estimação dos componentes de covariancia do arquivo MUEX2M.D ...................... 109 
Tabela 13b. Estimativas dos componentes de covariancia, herdabilidade, e correlacões genéticas, fenotfpicas e de meio ambiente para os dados do arquivo MUEX2M.D. Valores iniciais próximos às estimativas

Tabela 13c. Estimativas dos componentes de covariancia, herdabilidade e correlacões genéticas, fenotf́picas e de meio ambiente para os dados do arquivo MUEX2M.D. Valores iniciais sugeridos por

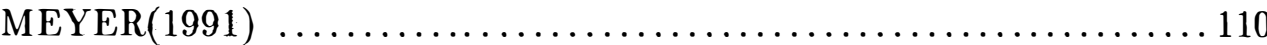

Tabela 13d. Estimativas dos componentes de covariância, herdabilidade e correlacões genéticas, fenotípicas e de meio ambiente para os dados do arquivo MUEX2M.D. Valores iniciais 10 vezes aos sugeridos por $\operatorname{MEYER}(1991)$

Tabela 13e. Estimativas dos componentes de covariancia, herdabilidade e correlacões genéticas, fenotípicas e de meio ambiente para os dados do arquivo MUEX2M.D. Análise realizada de acordo com a estratégia B . . . . 112

Tabela 13f. Informações adicionais referentes ao desempenho dos programas na análise dos dados do arquivo MUEX2M.D ................. 113

Tabela 14. Estimativas dos componentes de covariancia para os dados do arquivo SIMUL050.BEM. Análise realizada de acordo com a estratégia B . . . 115

Tabela 14a. Estimativas dos componentes de covariancia para os dados do arquivo SIMUL050.MIS. Análise realizada de acordo com a estratégia B

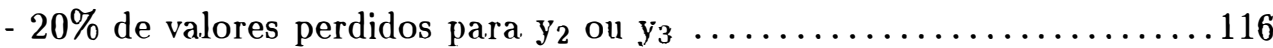

Tabela 14b. Estimativas de herdabilidade $\left(\hat{h}^{2}\right)$, repetibilidade $(\hat{r})$ e correlações genéticas $\left(\hat{r}_{g}\right)$, fenotípicas $\left(\hat{r}_{p}\right)$ e de meio ambiente $\left(\hat{r}_{e}\right)$ para os dados das Tabelas 14 e $14 \mathrm{a}$

Tabela 14c. Estimativas dos componentes de covariância para os dados do arquivo SIMUL050.PER. Análise realizada de acordo com a estratégia B - $20 \%$ de valores perdidos para $y_{2}$ ou $y_{3}$ perda total do registro $\ldots \ldots \ldots 118$

Tabela 14d. Estimativas dos componentes de covariancia para os dados do arquivo SIMUL050.SEL. Análise realizada de acordo com a estratégia $\mathbf{B}$ no $1^{\circ}$ parto $\mathrm{y}_{1}<3700$ unidades

Tabela 14e. Estimativas de herdabilidade $\left(\hat{h}^{2}\right)$, repetibilidade $(\hat{r})$ e correlações genéticas $\left(\hat{r}_{g}\right)$, fenotípicas $\left(\hat{r}_{p}\right)$ e de meio ambiente $\left(\hat{r}_{e}\right)$ para os dados

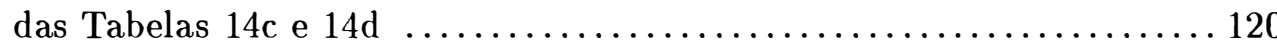

Tabela 15. Estimativas dos componentes de covariancia para os dados do arquivo SIMUL050.MAL. Análise realizada de acordo com a estratégia B . . 122

Tabela 15a. Estimativas dos componentes de covariância para os dados do arquivo SIMUL050.MAL. Valores iniciais iguais aos obtidos no programa

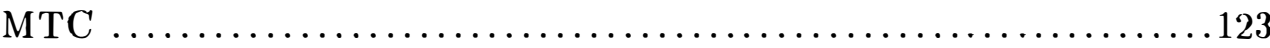

Tabela 15b. Estimativas dos componentes de covariância para os dados do arquivo SIMUL050.MAL. Valores iniciais iguais aos obtidos no programa

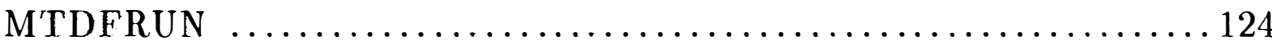

Tabela 15c. Estimativas dos componentes de covariância para os dados do arquivo SIMUL050.MAL Valores iniciais iguais aos obtidos no programa

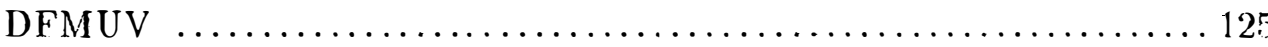


Tabela 16. Estimativas dos componentes de covariancia para os dados do arquivo SIMUL100.SEP. Valores iniciais iguais aos utilizados para simulacão - sem perda de dados

Tabela 16a. Estimativas dos componentes de covariancia para os dados do arquivo SIMUL100.PER. Valores iniciais iguais aos utilizados para simula-

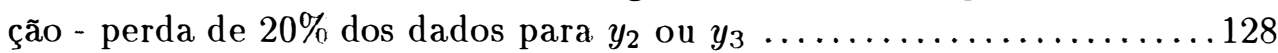

Tabela 16b. Estimativas de herdabilidade $\left(\hat{h}^{2}\right)$, repetibilidade $(\hat{r})$ e correlações genéticas $\left(\hat{r}_{g}\right)$, fenotípicas $\left(\hat{r}_{t}\right)$ e de meio ambiente $\left(\hat{r}_{e}\right)$ para os dados das Tabelas 16 e $16 \mathrm{a}$...

Tabela 16c. Estimativas dos componentes de covariancia, herdabilidade e correlações genéticas, fenotipicas e de meio ambiente para os dados do arquivo MULTI282.SEP. Análise realizada de acordo com a estratégia B . . . 130

Tabela 16d. Estimativas dos componentes de covariancia, herdabilidade e correlacões genéticas para os dados do arquivo MULTI615.SEP. Valores iniciais iguais aos valores utilizados para simulação

Tabela 17. Estimativas dos componentes de covariancia para os dados do arquivo SIMUL100.ARQ. Valores iniciais iguais aos valores utilizados para

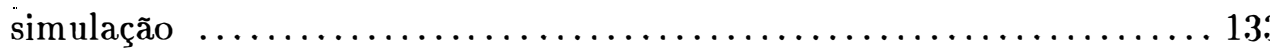

Tabela 17a. Estimativas dos componentes de covariancia para os dados do arquivo SIMUL100.ARQ. Valores iniciais 0.1 vezes os valores simulados . ...134

Tabela 17b. Estimativas dos componentes de covariancia para os dados do arquivo SIMUL100.PER. Análise realizada de acordo com a estratégia B

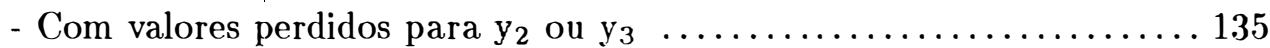

Tabela 17c. Estimativas de herdabilidade $\left(\hat{h}^{2}\right)$, repetibilidade $(\hat{r})$ e correlações genéticas $\left(\hat{r}_{g}\right)$, fenotípicas $\left(\hat{r}_{t}\right)$ e de meio ambiente $\left(\hat{r}_{e}\right)$ para os dados

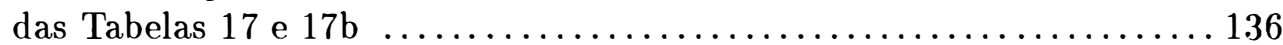

Tabela 18. Estimativas dos componentes de covariância para os dados do arquivo MULTI615.ARQ. Valores iniciais iguais aos simulados.

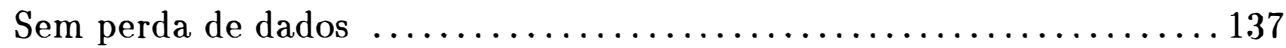

Tabela 18a. Estimativas de herdabilidade $\left(\hat{h}^{2}\right)$, repetibilidade $(\hat{r})$ e correlaçõesgenéticas $\left(\hat{r}_{g}\right)$, fenotípicas $\left(\hat{r}_{p}\right)$ e de meio ambiente $\left(\hat{r}_{e}\right)$ para os da-

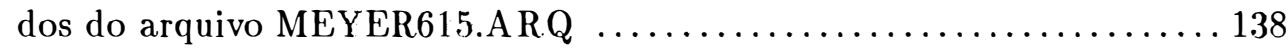

Tabela 18b. Estimativas dos componentes de covariancia para os dados do arquivo MULTI615.MIS. Valores iniciais iguais aos valores utilizados para simulação - $20 \%$ de valores perdidos para $y_{2}$ ou $y_{3} \ldots \ldots \ldots \ldots 139$

Tabela 18c. Estimativas de herdabilidade $\left(\hat{h}^{2}\right)$, repetibilidade $(\hat{r})$ e correlações genéticas $\left(\hat{r}_{g}\right)$, fenotípicas $\left(\hat{r}_{p}\right)$ e de meio ambiente $\left(\hat{r}_{e}\right)$ para os dados da Tabela 18b .......................................... 140

Tabela 19. Estimativas dos componentes de Covariancia para os dados do arquivo SIMUL100.ARQ. Valores iniciais baseados nos resultados das análises uni e bivariada - sem perda de dados .................... 141

Tabela 19a. Estimativas dos componentes de Covariancia para os dados do arquivo SIMUL100.ARQ. Valores iniciais baseados nos resultados das análises uni e bivariada perda de $20 \%$ dos dados para $y_{1}$ ou $y_{2} \ldots \ldots \ldots 142$ 
Tabela 19b. Estimativas de herdabilidade $\left(\hat{h}^{2}\right)$, repetibilidade $(\hat{r})$ e correlaçõesgenéticas $\left(\hat{r}_{g}\right)$, fenotfpicas $\left(\hat{r}_{p}\right)$ e de meio ambiente $\left(\hat{r}_{e}\right)$ para os dados

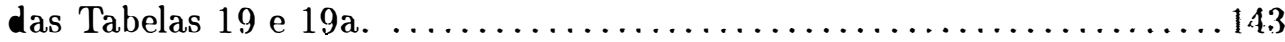

Tabela 20. Estimativas dos componentes de covariancia para os dados do arquivo CNPGL001.ARQ. Análise realizada de acordo com a estratégia B . . 145

Tabela 20a. Estimativas de herdabilidade $\left(\hat{h}^{2}\right)$ e correlações genéticas $\left(\hat{r}_{g}\right)$, fenotf́picas $\left(\hat{r}_{p}\right)$ e de meio ambiente $\left(\hat{r}_{e}\right)$ para os dados da Tabela $20 . \quad \ldots 146$ 
PROCEIIMENTOS NUMÉRICOS E ESTIMAÇÃO IDE C'OMPONEN'TES IDE COVARIÂNCIA EM ANALISE MULTIVARIAIA PELO MÉTOIOO I)A MAXIMA VEROSSIMILHANÇA RESTRITA - MOIELOS MISTOS APLICAIOOS AO MELHORAMENT'O ANIMAL,

\author{
Autor : Rui da Silva Verneque \\ Orientador : Prof. Cássio Roberto de Melo Godoi
}

\title{
RESUMO
}

Este trabalho tem por objetivos: a) descrever alguns procedimentos numéricos para obtenção de solução de sistemas de equações lineares, e para estimação de componentes de covariancia, em análise multivariada, aplicados à área de melhoramento animal; b) realizar uma avaliação comparativa entre os três sistemas de processamento de dados: DFREML (Derivative Free Restricted Maximum Likelihood (MEYER, 1993), MTC (Multitrait REML Estimation) (MISZTAL, 1992) e MTDFREML (Multiple Traits Derivative Free Restricted Maximum Likelihood) (BOLDMAN et al., 1993); e c) sugerir estratégias de análises, utilizando os programas em avaliação. Esses sistemas visam estimar componentes de covariância, adotando-se o método da máxima verossimilhança restrita (REML).

Realiza-se uma breve descrição dos métodos numéricos comumente empregados na solução de sistemas de equações lineares. São mostrados os métodos de maior aplicação na área de melhoramento animal e áreas afins.

Descrevem-se os principais métodos de estimação de componentes de covariância, deduzindo-se, na maioria dos casos, a forma de obtenção dos estimadores para cada um dos métodos.

Apresentam-se formas apropriadas para simulação de dados baseada em modelo animal, considerando-se ou não o parentesco entre indivíduos.

Estimam-se os componentes de variancia e covariancia a partir de dados cedidos por autores de diversas pesquisas; dados simulados e dados de campo, provenientes de registros de produção de leite de rebanhos da raça Gir leiteiro, extraidos do arquivo zootécnico nacional, mantido no CNPGL/EMBRAPA.

Foram simuladas até três características para cada experimento, admitindo-se diferentes modelos (com um ou dois fatores aleatórios além do erro) e várias matrizes de componentes de covariancia.

Os sistemas são rápidos e eficientes para estimação de variâncias em análise univariada. Em análise multivariada, os métodos livres de derivadas são eficientes para estimação de componentes de covariância, para o caso em que as características apresentem correlações não muito elevadas e quando os valores preliminares ou valores de partida representem uma boa aproximação das estimativas. Estes métodos, no entanto, não se mostram apropriados quando os valores iniciais utilizados são distantes das estimativas. Da 
mesma forma, para análise em que se inclui um número elevado de características (maior do que 3) e para situações em que a matriz de coeficientes do modelo linear misto não é esparsa, os métodos livres de derivadas, embora estimem adequadamente os parâmetros, tornam-se extremamente lentos. Por outro lado, estes sistemas são flexı́veis, permitindo adotar diferentes estratégias de estimação, para aumentar a velocidade e eficiência do processo. É possivel, por exemplo, executar uma análise univariada, seguida de análise multivariada, fixando-se alguns dos componentes. Posteriormente, adotando-se os parâmetros pré-estimados, pode-se realizar a análise multivariada repetidas vezes, sem se fixar qualquer componente. Este procedimento é denominado neste trabalho de estratégia. $\mathrm{B}$. Tal estratégia conduz a bons resultados, ou seja, é um procedimento que permite estimar componentes de covariância em menor tempo, menor número de iterações e de avaliações da verossimilhança.

O sistema MTC que adota o método REML e o algorftmo EM (maximização de esperanças), com transformação canônica do vetor de observações multivariadas, mostra-se muito eficiente para análise de modelos mistos com um único fator aleatório além do erro (modelo animal ou modelo de touro). No entanto, para modelos em que se incluem efeitos aleatórios adicionais (efeito permanente de meio, por exemplo), principalmente quando não há a diagonalização perfeita da matriz de componentes de covariancia $G$, este sistema fornece estimativas apenas aproximadas. Este também não permite análise de modelos com presença de efeito aleatório adicional correlacionado com animal (efeito materno, por exemplo) Além disso, em análise multivariada, o sistema exige igualdade de matriz de delineamento entre variáveis. Neste caso, a perda de um dado implica na perda total do registro. Trata-se de um método rápido para estimação de componentes de covariâncias e produz estimativas sempre no espaço de parâmetros.

Quando a convergência ocorre próximo ao limite do espaço de parâmetros ou quando se trata de superfícies muito planas, o método de Powell, adotado no sistema DFREML, apresenta frequientemente convergência irregular ou prematura, não sendo de uso indicado nestes casos. Nos demais casos, ele apresenta-se como um método eficiente.

O sistema MTDFREML mostra-se muito eficiente na procura de máximos da função de verossimilhança. No entanto, é o sistema que apresenta convergência mais lenta em análise multivariada, praticamente em todas situações avaliadas. Tal lentidão pode, em algumas situações, comprometer o uso deste, dependendo do tipo de modelo a ser analisado, número de equações e de características a serem avaliadas.

No geral, os sistemas em avaliação constituem-se em otimas ferramentas para estimação de componentes de covariância para modelos bem gerais, com grande número de classes de efeitos fixos e aleatórios. 


\title{
NUMERICAL PROCEDURES AND ESTIMATION OF COVARIANCE IN MULTIVARIATE ANALYSIS BY THE METHOD OF RESTRICTED MAXIMUM LIKELIHOOD - MIXED MODELS APPLIED TO ANIMAL BREEDING
}

\author{
Author: Rui da Silva Verneque \\ Adviser : Prof. Cássio R. de M. Godoi
}

\section{SUMMARY}

This work has as it's objectives: a) to describe some numerical procedures for obtaining solutions to linear equations and for the estimation of the components of covariance in multivariate analysis applied to animal breeding; b) to make a comparative evaluation of three systems of data processing: DFREML (Derivative Free Restricted Maximum Likelihood (MEYER, 1993), MTC (Multitrait REML Estimation) (MISZTAL, 1992) e MTDFREML (Multiple Traits Derivative Free Restricted Maximum Likelihood) (BOLDMAN et al., 1993); and c) to suggest strategies for analysis using the programs under evaluation. These systems purpose is to estimate covariance components using the restricted maximum likelihood method (REML).

A brief description is given of the numerical methods commonly used in the solution of linear equations. The methods of particular application to animal breeding and related areas are highlighted.

The principal methods for estimating the components of covariance are described, deducing, in the majority of cases, the form of obtaining the estimators for each of the methods.

Appropriated form of data simulation are presented, based on animal models, with and without consideration of the relationship between infividuals.

The components of variance and covariance are estimated from data ceded by the authors of various research projects, simulated data and field data, derived from production records of the milking Gir breed, extracted from the national animal production register, maintained at the National Dairy Cattle Research Center (CNPGL) of the Brazilian National Agricultural Research Organization (EMBRAPA).

Three traits were simulated in each experiment allowing for different models (with one or two random elements, apart from the error) and various matrices of the components of covariance.

The systems are rapid and efficient for the estimation of variances in univariate analysis. In multivariate analysis the derivative free methods are efficient for the estimation of the components of covariance in those cases in which the traits show correlations which are not very high and when the prior or starting values represent a good approximation of the estimates. These methods, however, are not appropriate when the prior values are far from the estimates. In the same way, for analyses including greater 
number of traits (more than three) and for situations in which a matrix of coefficients of the mixed linear model is not sparse, the derivative free methods, although they estimate the parameters adequately, become extremely slow. On the other hand these systems are flexible, allowing for the adoption of different strategies of estimation, so as to increase the velocity and efficiency of the process. It is possible, for example, to execute a univariate analysis, followed by a multivariate analysis, fixing some of the components. Subsequently, adopting the previously estimated parameters, one may run execute a multivariate analysis several times, without fixing any component. This procedure is called "Strategy B" in this work. Such a strategy leads to good results, that is to say it is a procedure which allows the estimation of the components of covariance in less time and with a smaller number of iterations and of evaluations of likelihood.

The MTC system, which uses the REML method and EM (expectation and maximization) algorithm, with canonical transformation of the vector of multivariate observation, shows itself very efficient with one random factor apart from the error (on animal model or sire model). However, for models in which additional random factors are included (the permanent effect of the environment, for example), mainly when there is not a perfect diagonalization of the matrix of components of $G$, this system provides estimates which are only approximated. Apart from that, in multivariate analysis, this system requires equality of the design matrix among variables. In this case, the loss of one trait implies the total loss of the record. It is, nevertheless, a rapid method for estimation of the components of covariance and produces estimates which are always within the parameter space.

When the convergence occurs close to the limit of the parameter space or when one is concerned with very flat surfaces, Powell's method, adopted in the DFREML system, frequently results in an irregular or premature convergence, not being indicated for use in such cases. In other cases it shows itself to be an efficient method.

The system MTDFREML shows itself very efficient in the search for the maximum of the likelihhod funcion. However, it is the system which presents the slowest convergence in multivariate analysis, in practically all the situations evaluated. Such slowness can, in some situations, inhibit the use of this system, depending on the type of model to be analysed, the number of eqations and traits of the system to be avaluated.

In general, the systems evaluated constitute excellent tools for the estimation of the components of covariance in many types of model, with a large number of classes of fixed and random effects. 


\section{INTRODUÇÃO}

O melhoramento genético de animais domésticos baseia-se no estudo de dados coletados em vários rebanhos, de uma ou mais raças, em diversos locais, geralmente fazendas ou granjas, por vários anos.

Nas avaliações dos valores genéticos dos indivíduos (matrizes, reprodutores, etc), são utilizados parâmetros genéticos (tais como herdabilidade, repetibilidade, correlações genéticas), os quais necessitam ser estimados com precisão.

A precisão das estimativas do valor genético pode ser melhorada pelo uso de grandes conjuntos de dados, pela redução ou remoção das influências não genéticas nos dados analisados, pela incorporação de dados de parentes, através da adoção de modelos mistos apropriados e, especialmente, pelo uso de um método numérico adequado para estimação dos componentes de covariância e para a previsão do valor genético do animal.

As análises estatísticas, realizadas com vistas à remoção das influências não genéticas, podem ser feitas adotando-se modelos adequados, que são, em geral, lineares uni ou multivariados, com efeitos fixos, aleatórios ou fixos e aleatórios (modelos mistos). Atualmente, tem-se adotado, na maioria dos trabalhos na área de melhoramento, o modelo animal, que, além de permitir realizar os ajustes para os efeitos fixos, considera os dados do próprio animal e de seus parentes (pela incorporação da matriz de parentesco), possibilitando, em uma única análise, realizar a avaliação do próprio indivíduo e dos invidíduos a ele relacionados.

Um problema comum associado ao uso de modelos mistos e aleatórios é a estimação de componentes de (co)variancia. A maioria das publicações de estimação de (co)variância é restrita às variâncias apenas. Com a ênfase que se tem dado à avaliação de características múltiplas, e em particular quando a seleção seqüiencial está envolvida e, consequientemente, nem todas as características são observadas em todos animais, existe a necessidade de se adotar métodos gerais para estimação de covariancias (HARVILLE, 1977).

Os componentes de covariâncias são importantes na obtenção da estatística $\mathrm{F}$, construção de índices de seleção, análise de modelos mistos com vistas à predição linear do tipo BLUP ("Best Linear Unbiased Predictor"), estimação de parâmetros genéticos, fenotípicos e de meio ambiente, planejamento de programas de melhoramento e interpretação do mecanismo genético de características quantitativas (HENDERSON, 1986).

São vários os métodos de estimação de componentes de variância e covariância, para características múltiplas, para dados não balanceados. Entre eles citam-se os métodos I, II e III de Henderson (HENDERSON, 1953); método dos estimadores não 
viciados de norma minima - MINQUE ("Minimum Norm Quadratic Unbiased Estimators") (RAO, 1971a); método da estimação não viesada de minima variância quadrática - MIVQUE ("Minimum Variance Quadratic Unbiased Estimation") (RAO, 1971b); método da máxima verossimilhança - ML ("Maximum Likelihood") (HARTLEY \& RAO, 1967); método da máxima verossimilhança restrita - REML ("Restricted Maximum Likelihood") (PATTERSON \& THOMPSON, 1971); método da falsa esperança (SCHAEFFER, 1986) e o método Bayesiano "VEIL" (Variance Estimation from Integrated Likelihoods) (GIANOLA \& FOULLEY, 1990).

Durante a última década, o método REML tem-se tornado o método preferido para estimação de componentes de covariancia em melhoramento animal ao fazer a partição das covariancias fenotípicas em covariâncias genéticas e outros componentes (MEYER, 1989 e 1991). Esta escolha deve-se não apenas às propriedades estatísticas desejáveis que o método apresenta, mas também à grande evolução dos recursos computacionais disponfveis, ao desenvolvimento e adaptação de algoritmos especializados, explorando características especificas da estrutura dos dados ou do modelo de análise, e à utilização de uma variedade de técnicas numéricas.

No passado, o método REML mostrou-se muito útil na análise de dados de gado de leite sob modelos de reprodutor ("sire model"). Por este modelo, os registros da progênie são utilizados somente na obtenção de informação da metade do valor genético de seu pai, enquanto que as informações da mãe e relacionamento entre fêmeas são ignorados. Recentemente, o interesse tem crescido pelo uso de um modelo mais detalhado, em particular pela forma simples de expressão dos valores genéticos, denominado "Modelo Animal" (AM), onde cada registro é usado para fornecer informação do mérito genético aditivo do animal (MEYER, 1991). Este tipo de modelo considera até mesmo os animais sem registros, em decorrência do relacionamento ou parentesco destes com os animais cujos registros estão sendo considerados.

A maioria das aplicações que utilizam o método REML são restritas a modelos com um único fator aleatório (exemplo reprodutor) além do erro, estimando somente dois componentes de variancia em análise univariada ou $p(p+1)$ componentes de covariancias, para análise multivariada de $\mathrm{p}$ características. Mais recentemente, no entanto, pela adoção de técnicas mais apuradas, o uso do método tem-se expandido para modelos multivariados, com vários fatores aleatórios.

Estimação de componentes de variancia pelo método REML, para dados não balanceados, geralmente requer esquemas iterativos. Livros-textos sobre análise númerica classificam os procedimentos para encontrar o ponto ótimo (mínimo ou máximo) de uma função conforme a quantidade de informação requerida da derivada da função (MEYER, 1989). Há, basicamente, três grupos: os denominados métodos de Newton que utilizam primeira e segunda derivadas, com convergência mais rápida; métodos que utilizam apenas primeira derivada, incluindo os métodos da máxima inclinação descendente ("Steepest descent"), gradiente conjugado e procedimentos Quasi-Newton, aproximando derivadas parciais de segunda ordem e, finalmente, os métodos livres de derivadas, envolvendo estratégias de procura direta ou aproximação numérica de derivadas.

Até recentemente, os principais algoritmos empregados nos trabalhos na

área de melhoramento animal, recaiam nas duas primeiras categorias. São eles o método 
Escore de Fisher (MSC) e o da maximização de esperanças (EM). O MSC é um caso especial dos procedimentos de Newton, requerendo valores esperados da segunda derivada do logarítmo da função de verossimilhança. Na segunda categoria, destaca-se o algorftimo EM (maximização de esperanças), descrito por DEMPSTER et al. (1977), que utiliza as informações da primeira derivada. Pela facilidade de se impor restrições às soluções quando se utiliza o algoritmo EM, o uso deste é defendido por vários autores, entre eles HENDERSON (1984a, 1984b); LAIRD et al. (1987) e MISZTAL (1990).

Por outro lado, a grande crítica que se faz ao uso do EM é que ele pode apresentar convergência extremamente lenta, mesmo nas situações em que outros métodos convergem rapidamente, como os de Newton-Raphson e Escore de Fisher . A razão para isto é que o algorítmo EM é um método de substituições sucessivas de primeira ordem e exibe convergência linear a.o término das iterações (LAIRD et al., 1987). Para contornar esta deficiência, LAIRD et al. (1987) sugerem o uso do método de aceleração da convergência de Aitken (veja, por exemplo, WESTLAKE, 1968 e CONTE, 1977), conjugado ao algoritmo EM. Tal procedimento faz com que a convergência do algorítmo EM passe de linear para quadrática, tornando-se muito mais rápida. Outro procedimento eficiente, conjugado ao algorftmo EM, é o uso de rotinas para operação com matrizes esparsas.

MEYER (1986) desenvolve o método denominado "Short-Cut" (SHC) combinando características do algoritmo EM para estimar os componentes dentro de classes do fator aleatório, com o método Escore de Fisher, para estimar os componentes entre as classes do fator aleatório. VALENTE (1988) desenvolve um conjunto de programas utilizando esse algorftmo. O algoritmo SHC tem a grande vantagem de apresentar convergência rápida, mas foi derivado apenas para situações particulares, como, por exemplo, modelos incluindo um único fator aleatório além do erro. Além disso, este método não garante estimativas no espaço de parâmetros.

Finalmente, um algoritmo REML livre de derivadas foi sugerido por GRASER et al. (1987) em análise univariada, para estimar os componentes de variancia dos efeitos genéticos aditivos e do erro, em um modelo animal. MEYER (1988, 1989, 1991 e 1993) e BOLDMAN \& VAN VLECK (1991) e BOLDMAN et al. (1993) estenderam o método para o campo multivariado, para modelos com dois ou mais fatores aleatórios.

$\mathrm{O}$ uso do método livre de derivadas é deduzido a partir da simplificação do logarıtmo da função de verossimilhança restrita da distribuição normal, apresentado por SEARLE (1971 e 1979) e HARVILLE (1977).

Para estimação dos componentes de variancia, GRASER et al. (1987) executam a eliminação de Gauss, em uma matriz formada pela junção da matriz de coeficientes. com o lado direito das equações normais, do modelo linear misto e adotam o procedimento de procura direta, por aproximação quadrática da verossimilhança. MEYER $(1989,1991$ e 1993$)$ adota o mesmo procedimento no caso de procura unidimensional do máximo da verossimilhança.

Para análise multivariada, MEYER (1989, 1991 e 1993) adota a eliminação de Gauss e os métodos simplex ou de Powell, respectivamente, na procura sequiencial ou linear do máximo da função de verossimilhança restrita. Por outro lado, BOLDMAN \& VAN VLECK (1991) e BOLDMAN et al. (1993) utilizam a decomposição de Cholesky da matriz de coeficientes, o método simplex, para procura seqüencial do máximo da função 
de verossimilhança e operação com matrizes esparsas. Este mesmo procedimento também é adotado em MEYER (1993).

De posse de todo um conjunto relativamente amplo de metodologias para estimação de componentes de covariancias, mas dadas as dificuldades computacionais existentes na implementação destes métodos, MEYER (1988, 1989, 1991 e 1993) desenvolve um sistema denominado DFREML ("Derivative Free Restricted Maximum Likelihood"). O sistema é implantado para estimação de componentes de covariância, valores genéticos e outros processos relacionados, para análise uni ou multivariada, com dois ou mais fatores aleatórios, com ou sem valores perdidos para alguma característica.

Ao mesmo tempo, BOLDMAN \& VAN VLECK (1991) apresentam um artigo mostrando a eficiência da decomposição de Cholesky, da matriz de coeficientes, na estimação dos componentes de covariância. A ssim, BOLDMAN et al. (1993), aproveitando parte do trabalho de Meyer, desenvolvem rotinas especificas para a imposição de restrições paramétricas ao sistema e utilizam a decomposição de Cholesky de matrizes positivas definidas e acoplam o conjunto de rotinas da SPARSPAK (GEORGE et al., 1980), e criam o sistema MTDFREML ("Multiple Trait Derivative Free Restricted Maximum Likelihood").

Também MISZTAL (1993) escreve e implementa outro conjunto de programas denominado MTC ("Multitrait REML Estimation"), adotando, para estimação de componentes de covariância, o método REML, e utiliza o algorftmo EM, combinado com o método de aceleração da convergência de Aitken, acoplado ao conjunto de rotinas FSPAK (PEREZ-ENCISO \& MISZTAL, 1992), para inversão de matrizes esparsas.

O desenvolvimento dos sistemas DFREML, MTDFREML e MTC, bem como as metodologias a eles acopladas, como os algoritmos de aceleração de convergência (método de aceleração da convergência de Aitken), inversão de matrizes esparsas, eliminação de Gauss, método simplex e de Powell, entre outros, possibilita decréscimo da ordem de até 900 vezes no tempo gasto para a estimação de componentes de covariância, em comparação aos procedimentos anteriormente utilizados. Além disso, possibilitam a realização de análises de grandes conjuntos de dados, com número elevado de classes de efeitos fixos e aleatórios. Enfim, os sistemas em discussão permitem um aumento considerável no tamanho e complexidade de problemas analisáveis, utilizando o método REML.

Embora a literatura consultada seja farta em informações pertinentes aos métodos de estimação de componentes de variância, não existe conhecimento acumulado e suficiente quanto à comparação das técnicas de estimação de componentes de covariância em análise multivariada adotando-se o modelo animal e o método REML. Isto se observa especialmente porque, até por volta de 1988, os poucos programas de computador existentes utilizando o método REML adotam o modelo de um fator aleatório além do erro (na área de gado de leite, comumente chamado modelo de reprodutor). Somente a partir de 1988 são desenvolvidos os programas DFREML, MTDFREML e MTC, ainda insuficientemente testados.

Por outro lado, considerando-se que a precisão das estimativas dos componentes de covariância é fundamental para o processo de desenvolvimento cientifico e tecnológico, especialmente aqueles relacionados à estimação de parâmetros genéticos e fenotfpicos, essenciais para a avaliação genética de animais, há a necessidade de se obter informações pormenorizadas e se possivel conclusivas a respeito das técnicas de estimação 
de componentes de covariancia, em análise multivariada, pelo método REML

\section{Resumindo, os objetivos deste trabalho são:}

1. Descrever as técnicas numéricas e os principais métodos de estimação de componentes de covariância na análise uni e multivariada, pelo método REML.

2. Realizar uma análise comparativa entre os sistemas DFREML - "Derivative Free Restricted Maximum Likelihood" (MEYER, 1993), MTDFREML - "Multiple Trait Derivative Free Restricted Maximum Likelihood" (BOLDMAN et al., 1993) e MTC - "Multitrait REML Estimation" (MISZTAL, 1993), com dados simulados, verificando-se velocidade de convergência e comportamento sob diferentes valores iniciais.

3. Aplicação dos programas em dados de campo, referentes à produção de leite de rebanhos da raça Gir Leiteiro.

4. Sugerir estratégias de análise, utilizando os programas em consideração. 


\section{REVISÃO DE LITERATURA}

\subsection{Procedimentos Numéricos para Resolução de Sistemas de Equações Lineares}

Os procedimentos numéricos para resolução de sistemas de equações lineares podem ser divididos em duas classes: os métodos diretos e os métodos indiretos ou iterativos. Os métodos diretos produzem soluções exatas em um número finito de operações, se não houver erros de arredondamento. São indicados quando o número de equações envolvidas não é muito grande. Por outro lado, os processos iterativos iniciam com uma solução aproximada e obtém-se uma solução melhorada a cada passo do processo iterativo, mas requerem um número infinito de passos para obter uma solução exata, na ausência de erros de arredondamento. A precisão da solução depende do número de iterações realizadas e da precisão do programa ou da máquina.

Serão apresentados, de uma forma resumida, a descrição de alguns procedimentos numéricos adotados regularmente na obtenção de soluções de sistemas de equações lineares do tipo $A X=B$, ou $A x=b$, para $A$ uma matriz n x n, com vistas à estimação de componentes de covariancia, deduzidas a partir de um modelo linear misto uni ou multivariado. Alguns destes procedimentos são utilizados nos trabalhos de MISZTAL \& GIANOLA (1987); MEYER (1988, 1989, 1991 e 1993); BOLDMAN \& VAN VLECK (1991), VAN VLECK \& GREGORY (1992) e BOLDMAN et al. (1993), entre outros.

\subsubsection{Métodos Baseados na Triangulação}

Segundo WESTLAKE (1968), os procedimentos baseados na triangulação, bem como aqueles baseados na diagonalização são provavelmente os mais úteis, pois eles aplicam-se a qualquer tipo de matriz, são fáceis de programar e apresentam ótima performance quanto à precisão e velocidade. Qualquer biblioteca de programas deve conter ao menos um desses métodos.

Baseiam-se no fato de que qualquer matriz quadrada $A$ é linha-equivalente a uma matriz triangular e esta forma triangular é obtida por um número finito de operações elementares nas linhas e/ou colunas de $A$. Se $A$ é real, então a matriz triangular resultante é também real.

Todos procedimentos de triangulação são baseados na decomposição $L D U$ ou $L U$, em que $A$ pode ser expressa (ou rearranjada) como $A=L D U$, para $L$ uma matriz triangular inferior, $D$ diagonal e $U$ triangular superior, para $A$ não singular. A única diferença entre os métodos centra-se na maneira pela qual $D$ é particionada. As vezes, $D$ 
pode ser incorporada total ou parcialmente em $L$ ou $U . D$ pode também ser particionada de forma que os termos diagonais de $L$ e $U$ sejam iguais em valor absoluto.

Suponha que $A=L D U$ é definida sem especificar se $D$ é incorporada à $L$ ou $U$, ou ambas. Então

$$
\left(\begin{array}{cccc}
l_{11} & 0 & \cdots & 0 \\
l_{21} & l_{22} & \cdots & 0 \\
\vdots & \vdots & \vdots & \vdots \\
l_{n 1} & l_{n 2} & \cdots & l_{n n}
\end{array}\right)\left(\begin{array}{cccc}
u_{11} & u_{12} & \cdots & u_{1 n} \\
0 & u_{22} & \cdots & u_{2 n} \\
\vdots & \vdots & \vdots & \vdots \\
0 & 0 & \cdots & u_{n n}
\end{array}\right)=\left(\begin{array}{cccc}
a_{11} & a_{12} & \cdots & a_{1 n} \\
a_{21} & a_{22} & \cdots & a_{2 n} \\
\vdots & \vdots & \vdots & \vdots \\
a_{n 1} & a_{n 2} & \cdots & a_{n n}
\end{array}\right)
$$

Desejamos resolver o sistema de equações para $l_{i j}$ e $u_{i j}, i \leq j$, com $a_{i j}$ conhecido. Após a multiplicação das duas matrizes $L$ e $U$ e igualando os resultados aos correspondentes elementos de $A$, obtêm-se as equações:

$$
\begin{aligned}
\sum_{k=1}^{\min (i, j)} l_{i k} u_{k j} & =a_{i j} \\
l_{11} u_{11} & =a_{11} \\
l_{11} u_{12} & =a_{12} \\
& \vdots \\
l_{11} u_{1 n}= & a_{1 n} \\
l_{21} u_{11}=a_{21} & l_{r 1} u_{11}=a_{r 1} \\
l_{21} u_{12}+l_{22} u_{22}=a_{22} \quad & l_{r 1} u_{11}=a_{r 1} \\
\vdots & \vdots \\
\vdots & l_{r 1} u_{1 r}+l_{r 2} u_{2 r}+\ldots+l_{r r} u_{r r}=a_{r r} \\
\vdots & \vdots \\
l_{21} u_{1 n}+l_{22} u_{2 n}=a_{2 n} & l_{r 1} u_{1 n}+l_{r 2} u_{2 n} \ldots l_{r r} u_{r n}=a_{r n}
\end{aligned}
$$

Desde que existem $n^{2}+n$ elementos desconhecidos e somente $n^{2}$ equações, $\mathrm{n}$ dos elementos desconhecidos podem ser livremente especificados. Existem métodos nos quais os elementos diagonais de $L$ ou de $U$ são supostos iguais a 1 , outros métodos supõem-se que os elementos diagonais de $L$ ou $U$ são iguais a $\mathrm{k}(k \neq 0)$. Alguns dos métodos dependem explicitamente da resolução triangular, no sentido de que todos os elementos de $L$ e de $U$ são computados, e, em outros métodos, uma das matrizes $L$ ou $U$ não é explicitamente computada.

O principal erro que ocorre da solução do sistema $A X=B$ por triangulação pode estar mais no próprio processo de decomposição de $A$ por $L D U$ do que da solução dos dois sistemas triangulares. 


\subsubsection{Sistema Triangular}

A solução de um sistema triangular superior ou inferior da forma $U X=$ $B$, em que $U$ é triangular superior, é obtida como:

$$
x_{i j}=\frac{1}{u_{i i}}\left(b_{i, j}-\sum_{k=i+1}^{n} u_{i k} x_{k j}\right) \text { para } \mathrm{j}=1,2, \ldots, \mathrm{r} \text { e } \mathrm{i}=\mathrm{n}, \mathrm{n}-1, \ldots, 1 .
$$

Quando $B=I, U^{-1}=\left(x_{i j}\right)$ é obtido, para $x_{i i}=\frac{1}{u_{i i}}, \mathrm{e}$

$$
x_{i j}=\frac{1}{u_{i i}}\left(-\sum_{k=i+1}^{j} x_{k j}\right)
$$

também para $\mathrm{j}=1,2, \ldots, \mathrm{r}$ e $\mathrm{i}=\mathrm{n}, \mathrm{n}-1, \ldots, 1$.

O sistema $L X=B$, em que $L$ é triangular inferior, é resolvido como:

$$
\begin{gathered}
x_{i j}=\frac{1}{l_{i i}}\left(b_{i j}-\sum_{k=1}^{i-1} l_{i k} x_{k j}\right) \text { para } \mathrm{j}=1,2, \ldots, \mathrm{r} \text { e } \mathrm{i}=1 \\
\text { Quando } B=I, L^{-1}=\left(x_{i j}\right) \text { é obtido, para } x_{i i}=\frac{1}{l_{i i}}, \mathrm{e} \\
x_{i j}=\frac{1}{l_{i i}}\left(-\sum_{k=j}^{i-1} x_{k j}\right)
\end{gathered}
$$

também para $\mathrm{j}=1, \ldots, \mathrm{r} \quad \mathrm{e} \quad \mathrm{i}=1, \ldots, \mathrm{n}$

\subsubsection{Eliminação de Gauss ou Condensação Pivotal}

Por este método são realizadas operações elementares em $A$ (e em $B$ ) para zerar elementos sucessivos de $A$, de tal maneira a reduzir $A$ a uma matriz $U$ triangular superior, permitindo a obtenção direta do último elemento desconhecido de $X$. O produto das matrizes resultante das operações elementares nas linhas de $A$ é uma matriz triangular inferior $L$ com elementos da diagonal iguais a 1. Então $L A=U$ ou $A=L^{-1} U$. Desde que $L^{-1}$ é novamente triangular inferior, $A$ é expressa na forma $L D U$ com $D$ inclurda em $U$. $U$ é obtida explicitamente, mas $L$ e $L^{-1}$ não o são.

$\mathrm{O}$ processo desenvolve-se da seguinte forma: No $k^{\text {esimo }}$ estágio, um elemento pivô $a_{i j} \neq 0$ de $A^{(k-1)}$ é escolhido conforme algum critério. Então a $i^{\text {esima }}$ e a $k^{\text {esima }}$ linhas e a $j^{\text {esima }}$ e a $k^{\text {esima }}$ colunas de $A^{(k-1)}$ são trocadas, de forma que $a_{i j}^{(k-1)}$ torna-se $a_{k k}^{(k)} . A$ $i^{\text {ésima }}$ e a $k^{\text {ésima }}$ linhas de $B^{(k-1)}$ são também trocadas. O pivô $a_{k k}^{(k)}$ é então utilizado para obter zeros em todas posições nas colunas abaixo da diagonal. Os elementos transformados são dados por:

$$
a_{i j}^{(k)}=a_{i j}^{(k-1)} \quad \text { para } \mathrm{i}=1, \ldots, \mathrm{k} \text { e } \mathrm{j}=\mathrm{i}, \ldots, \mathrm{n},
$$




$$
\begin{aligned}
& b_{i j}^{(k)}=b_{i j}^{(k-1)} \quad \text { para } \mathrm{i}=1, \ldots, \mathrm{k} \text { e } \mathrm{j}=\mathrm{i}, \ldots, \mathrm{r}, \\
& a_{i j}^{(k)}=a_{i j}^{(k-1)}-\left(\frac{a_{i k}^{(k-1)}}{a_{k k}^{(k-1)}}\right) a_{k j}^{(k)} \quad \text { para } \mathrm{i}=\mathrm{k}+1, \ldots, \mathrm{n} \text { e } \mathrm{j}=\mathrm{k}, \ldots, \mathrm{n}, \\
& b_{i j}^{(k)}=b_{i j}^{(k-1)}-\left(\frac{a_{i k}^{(k-1)}}{a_{k k}^{(k-1)}}\right) b_{k j}^{(k)} \quad \text { para } \mathrm{i}=\mathrm{k}+1, \ldots, \mathrm{n} \text { e } \mathrm{j}=1, \ldots, \mathrm{r}, \mathrm{e} \\
& a_{i j}^{(k)}=0 \quad \text { para } \mathrm{j}=1, \ldots, \mathrm{k}-1 \quad \text { e } \mathrm{i}=\mathrm{j}+1, \ldots, \mathrm{n}
\end{aligned}
$$

Supondo que no estágio $\mathrm{k}=0$ têm-se as matrizes $A$ e $B$ originais, a forma triangular desejada é obtida na conclusão do estágio $\mathrm{k}=\mathrm{n}-1$. O sistema triangular superior resultante é resolvido como em ((1), pág. 7). A substituição reversa fornece:

$$
x_{i j}=\frac{1}{a_{i i}^{n-1)}}\left(b_{i j}^{(n-1)}-\sum_{k=i+1}^{n} a_{i k}^{(n-1)} x_{k j}\right) \text { para } j=1, \ldots, r \text { e } i=n, n-1, \ldots, 1 .
$$

A troca entre as colunas $\mathrm{j}$ e $\mathrm{k}$ de $A^{(k-1)}$, realizada no estágio $\mathrm{k}$, para colocar o pivô na posição diagonal $(\mathrm{k}, \mathrm{k})$, tem o efeito de trocar as variáveis $x_{j}$ e $x_{k}$ em cada coluna de soluções correspondentes a colunas de $B$. Por isto, ao final do processo, as linhas $\mathrm{j}$ e $\mathrm{k}$ de $X$ devem ser permutadas e de forma similar para quaisquer outras colunas trocadas em $A$. Linhas trocadas em $A$ o são também em $B$, de modo que correções adicionais não serão necessárias.

\section{- Seleção de Pivôs}

Usualmente são três os métodos de seleção de pivôs:

a) Sem posicionamento para tamanho

Escolhe-se $a_{k k}^{(k-1)}$, o $k^{\text {esimo }}$ elemento da diagonal da matriz $A$, como o elemento pivô do $k^{e s i m o}$ estágio do processo, após (k-1) transformações terem sido realizadas. Em consequiência de não haver neste caso trocas de linhas e nem trocas de colunas e não se fazer teste para tamanho, este procedimento torna-se o mais simples e rápido. Em conseqüência, não se dando atenção para tamanhos relativos dos elementos, erros de arredondamento não são minimizados. Em casos de $a_{k k}^{(k-1)}$ nulo, alguns elementos não nulos na mesma coluna podem ser usados como pivô. Neste caso há necessidade da troca da linha correspondente.

b) Posicionamento parcial para tamanho

Escolhe-se o pivô no $k^{\text {esimo }}$ estágio como sendo o elemento de maior valor absoluto na coluna $\mathrm{k}$ e linha $\mathrm{k}$ até $\mathrm{n}$. Neste caso, as linhas permutadas são necessárias para 
colocar o pivô na $k^{\text {esima }}$ linha. O teste para tamanho e a troca de linhas requerem maior tempo de computação, mas alguma melhoria em precisão deve ser observada.

c) Completo posicionamento para tamanho

No $k^{\text {esimio }}$ estágio, escolhe-se o pivô como o elemento de maior valor absoluto na submatriz composta das colunas $\mathrm{k}$ até $\mathrm{n}$ e linhas $\mathrm{k}$ até $\mathrm{n}$. Desde que não é possivel encontrar dois elementos pivô na mesma linha ou coluna, o número de elementos eleitos como pivô é reduzido após cada estágio. Ambas linha e coluna permutadas podem ser necessárias para levar o pivô para a $k^{\hat{e s i m a}}$ diagonal. Estas trocas juntas com o teste para tamanho dos elementos consomem tempo, mas erros de arredondamento são reduzidos consideravelmente.

Este método tem tido uso frequiente na área de melhoramento animal, no cálculo de componentes de covariância, pelo método livre de derivadas. Para isto, é montada uma matriz $M$, que é a matriz de coeficientes das equações do modelo linear misto (MME), aumentada pelo lado direito das mesmas equações e o vetor de formas quadráticas das observações, comumente referida como matriz do modelo misto (MMM). Aplicando-se a eliminação de Gauss com pivoteamento diagonal em $M$, ao final do processo, resulta na obtenção dos termos mais diff́ceis de ser obtidos por processo normal.

O algorítmo é apresentado por GRASER et al. (1987), para estimação de componentes de variância, e é espandido por MEYER $(1988,1989,1991$ e 1993), para o caso multivariado.

\subsubsection{Métodos compactos}

\subsection{Método Crout (ou Cholesky Geral)}

O método depende explicitamente da resolução triangular $A=L U$, isto é, os elementos de $L$ e $U$ são todos computados e utilizados. O termo compacto ou esquema compacto deve-se ao fato de que os elementos da forma triangular final são obtidos por acumulação, dispensando o cálculo e armazenamento de coeficientes $a_{i j}^{(k)}$ intermediários, reduzindo erros de arredondamento.

O método utiliza uma ordem fixa de eliminação e deve ser aplicado apenas quando $A$ for não singular e rearranjada para permutar linhas e colunas, de modo que todos os menores principais sejam não singulares.

Seja $A=L U$ as equações para os elementos de $L$ e $U$ da seção 2.1.1.1 (Pág.

8). Então

$$
\sum_{k=1}^{\min (i, j)} l_{i k} u_{k j}=a_{i, j} .
$$

Fazendo $u_{k k}=1$ para $\mathrm{k}=1,2, \ldots, \mathrm{n}$, tem-se $n^{3}$ equações e $n^{2}$ parâmetros desconhecidos, que produzem a seguinte decomposição, para $\mathrm{k}=1,2, \ldots, \mathrm{n}$ :

$$
u_{k k}=1
$$




$$
\begin{aligned}
l_{i k} & =a_{i k}-\left(\sum_{m=1}^{k-1} l_{i m} u_{m k}\right) \text { para } \mathrm{i}=\mathrm{k}, \ldots, \mathrm{n} \\
u_{k j} & =\frac{1}{l_{k k}}\left(a_{k j}+\sum_{m=1}^{k-1} l_{k m} u_{m j}\right) \text { para } \mathrm{j}=\mathrm{k}+1, \ldots, \mathrm{n} \\
l_{i k} & =0 \text { para } i<k \\
u_{k j} & =0 \text { para } j<k .
\end{aligned}
$$

Então, a ordem de cálculo é a primeira coluna de $L$ e primeira linha de $U$, segunda coluna de $L$, segunda linha de $U$, e assim por diante. Note que $l_{k k}=0$ se $|A|=$ 0 .

Como os elementos $l_{i k}$ e $u_{k j}$ são calculados, eles podem ser "postos" "sobre" a matriz $A$. Após obtenção dos elementos de $L$ e $U$, resolve-se $A X=B$ escrevendo $L U X=$ $B$, que é equivalente a resolver o sistema triangular $L \varepsilon=B$ para $\varepsilon$ e então $U X=\varepsilon$ para $X$. Assim,

$$
\begin{aligned}
& \varepsilon_{i j}=\frac{1}{l_{i i}}\left(b_{i j}+\sum_{k=1}^{n-1} l_{i k} \varepsilon_{k j}\right), \text { para } \mathrm{j}=1,2, \ldots, \mathrm{r} \text { e } \mathrm{i}=1, \ldots, \mathrm{n} \\
& x_{i j}=\left(\varepsilon_{i j}-\sum_{k=i+1}^{n} u_{i k} x_{k j}\right) \text { para } \mathrm{j}=1,2, \ldots, \mathrm{r} \text { e } \mathrm{i}=\mathrm{n}-1, \ldots, 1 .
\end{aligned}
$$

Para encontrar $A^{-1}$, obtém-se $A=L U$, tal que $A^{-1}=U^{-1} L^{-1} \cdot U^{-1}$ e $L^{-1}$ podem ser facilmente obtidos da seção 2.1.1.1.

A precisão do método pode ser melhorada se for introduzido o pivoteamente parcial. Após a coluna $l_{i k}, \mathrm{i}=\mathrm{k}, \ldots, \mathrm{n}$ ser computada, o maior $l_{i k}$ em valor absoluto, digamos $l_{j k}$, pode ser selecionado como $l_{k k}$ e esta linha $\left(a j^{\text {esima}}\right)$ permutada com a $k^{\text {esima }}$ linha em $L$ e $A$. Isto não deve causar problemas mesmo quando $L$ e $U$ sobrepuserem $A$. Então, a próxima linha de $U, u_{k j}$ para $\mathrm{j}=\mathrm{k}+1, \ldots, \mathrm{n}$ é computada. Sempre que uma nova coluna de $L$ for calculada, o maior de seus elementos em valor absoluto é assumido como o elemento diagonal.

As matrizes $L$ e $U$, assim obtidas, não representam a decomposição triangular de $A$, mas sim a decomposiçã॰ de $\tilde{\mathrm{A}}=\left(I_{i_{n}, n}, \ldots I_{i_{2}, 2} I_{i_{1}, 1}\right) A$, obtida de $A$, pela sequência de linhas permutadas, $I_{k}, k$ da linha $I_{k}$ com a $k^{\text {esima }}$ linha, para $\mathrm{k}=1, \ldots, \mathrm{n}$. Então, $\tilde{A}=L U$. Se for de interesse obter $A^{-1}$, calcula-se $\tilde{A}^{-1}=U^{-1} L^{-1}$. e como $\tilde{A}^{-1}=$ $A^{-1}\left(I_{i_{n}, n}, \ldots, I_{i_{2}, 2} I_{i_{1}, 1}\right), \Rightarrow A^{-1}=\tilde{A}^{-1}\left(I_{i_{n}, n}, \ldots, I_{i_{2}, 2} I_{i_{1}, 1}\right)$

As matrizes $I_{i_{k}}, k$ agem como colunas permutadas em $\tilde{A}^{-1}$ desde que elas tornaram-se multiplicadores do lado' direito do sistema de equações.

Seja a solução do sistema $A X=B$ pelo método Crout, com pivoteamento parcial. Define-se $\tilde{A}$ como acima e $\tilde{B}=\left(I_{i_{n}, n}, \ldots, I_{i_{2}, 2} I_{i_{1}, 1}\right) B$. Por esta razão, qualquer linha trocada em $L$ ou $A$ deve também ser permutada em $B$. Então, tem-se:

$$
\tilde{A} \tilde{X}=\tilde{B} \Rightarrow \tilde{X}=\tilde{A}^{-1} \tilde{B}=A^{-1}\left(I_{i_{n}, n}^{-1}, \ldots, I_{i_{2}, 2}^{-1} I_{i_{1}, 1}^{-1}\right)\left(I_{i_{n}, n}, \ldots, I_{i_{2}, 2} I_{i_{1}, 1}\right) B \mathrm{e}
$$

$\tilde{X}=A^{-1} B=X$. Assim, o sistema $\tilde{A} \tilde{X}=\tilde{B}$ pode ser resolvido por $\tilde{X}$ e esta é a solução, $X$, de $A X=B$. Não há necessidade de troca de colunas. Maiores detalhes podem ser vistos, por exemplo, em WESTLAKE(1968); PRESS et al. (1992) e MACHADO (1993). 


\subsection{Decomposição QR}

Dada uma matriz quadrada $A$ (o processo pode ser utilizado para matrizes triangulares gerais), a decomposição $\mathrm{QR}$ consiste em obter $Q$ e $R$, em que $R$ é uma matriz triangular superior e $Q$ ortogonal, isto é, $Q^{\prime} Q=I$, tal que $A$ possa ser decomposta como:

$$
A=Q \cdot R \text {. }
$$

Para a solução de sistemas de equações lineares da forma $A x=b$, primeiramente forma-se $Q^{\prime} b$ e então resolve-se

$$
R x=Q^{\prime} b, \quad \text { por retrosubstituição. }
$$

Desde que a decomposição $Q R$ envolve o dobro de operações em relação a decomposição $L U$, ela não é regularmente utilizada para solução de sistemas de equações lineares típicos. Entretanto, ela torna-se de aplicação recomendada em casos especiais como na solução de uma sucessão de sistemas de equações lineares em que cada um dos sistemas difere muito pouco do seu predecessor. Neste caso, a aplicação da decomposição $L U$ é complicada por exigir pivoteamento.

O algorftmo padrão para a decomposição $Q R$, envolve sucessivas transformações de Householder (PRESS et al., 1992). A matriz de Householder é dada por 1 $u \otimes u / 0.5 u^{\prime} u$. Uma matriz de Householder apropriada, aplicada à matriz dada, pode zerar todos elementos em uma coluna da matriz, situada abaixo do elemento escolhido. Desta forma, arraja-se a primeira matriz de Householder $Q_{1}$, para zerar os elementos da primeira coluna de $A$, abaixo do primeiro elemento. Similarmente $Q_{2}$ zera todos elementos da segunda coluna, abaixo do segundo elemento, e assim por diante, até $Q_{n-1}$. Desta forma,

$$
R=Q_{n-1} Q_{n-2} \ldots Q_{1} \cdot A .
$$

Desde que as matrizes de Householder são ortogonais, então

$$
Q=\left(Q_{n-1} \ldots Q_{1}\right)^{-1}=Q_{1} \ldots Q_{n-1}
$$

\subsection{Decomposição de Cholesky}

O método da raiz quadrada ou decomposição de Cholesky, aplica-se para matrizes simétricas. Seja $A$ uma matriz simétrica, isto é, $A=A^{\prime}$. Então $A$ pode ser decomposta (veja, por exemplo, WESTLAKE, 1968; SEARLE, 1982 e MACHADO, 1993) como

$$
A=M D M^{\prime}=\left(M D^{1 / 2}\right)\left(D^{1 / 2} M\right)^{\prime}=L L^{\prime}=U U^{\prime}
$$

para $M$ uma matriz triangular inferior, $D$ uma matriz diagonal e $L$ e $U$ matrizes triangulares inferior e superior, respectivamente.

Tomando-se $a_{i j}=a_{j i}$ e $l_{i j}=u_{j i}$ nas equações $\sum_{k=1}^{\min (i, j)} l_{i k} u_{k j}=a_{i j}$, na seção (2.1.1.3.1), tem-se que $\sum_{k=1}^{\min (i, j)} u_{k i} u_{k j}=a_{i j}$. Os elementos de $U$ são dados por:

$$
u_{11}=\sqrt{a}_{11}
$$




$$
\begin{aligned}
u_{1 j} & =\frac{a_{1 j}}{u_{11}} \quad \operatorname{para} \mathrm{j}=2, \ldots, \mathrm{n} \\
u_{i i} & =\left(a_{i i}-\sum_{k=1}^{i-1} u_{k i}^{2}\right)^{1 / 2} \text { para } \mathrm{i}=2, \ldots, \mathrm{n} \\
u_{i j} & =\frac{1}{u_{i i}}\left(a_{i j}-\sum_{k=1}^{i-1} u_{k i} u_{k j}\right) \text { para } \mathrm{j}=\mathrm{i}+1, \ldots, \mathrm{n} \text { e } \mathrm{i}=2, \ldots, \mathrm{n}
\end{aligned}
$$

Então $A X=U^{\prime} U X=B$ e $X=\left(U^{-1}\right)\left(U^{-1}\right)^{\prime} B$ e $U^{-1} \quad$ é facilmente obtida.

A decomposição é normalmente adotada para matrizes simétricas positivas definidas, mas aplica-se a matrizes simétricas, mesmo se $A$ não for positiva definida. No entanto, neste caso, os elementos diagonais de $U$ podem ser números complexos. Nenhum pivoteamento é necessário no caso de $A$ ser simétrica e positiva definida. Se $A$ é simétrica, mas não é positiva definida, o pivoteamento deve ser utilizado.

O método de Cholesky possui excelentes propriedades de economia de memória e estabilidade numérica (MACHADO, 1993). Trata-se de uma técnica matricial envolvida em muitos avanços em estratégias computacionais para a área de melhoramento animal, como regras para cálculo da inversa da matriz de coeficientes de parentesco, simulação de características multivariadas, transformação canônica para análise de caracteristicas múltiplas e transformação seqüencial para padronizar as variancias residuais para uma classe de análises de características múltiplas, com seleção cronológica (POLLAK \& QUAAS, 1982, WALTER \& MAO, 1985, BOLDMAN \& VAN VLECK, 1991 e MACHADO, 1993). Além disso, o método pode ser utilizado para solução de sistema de equações normais, com matriz de coeficientes simétrica e positiva definida, em casos de modelo de regressão ou em modelos de análise de variância após a imposição de restrições não estimáveis.

MACHADO (1993), citando Thisted (1988), comenta que todos os cálculos de uma análise de regressão podem ser realizados através da decomposição de Cholesky. Entretanto, por motivos mais conceituais do que computacionais, existem métodos de maior estabilidade numérica, como, por exemplo, decomposição de Householder e, dentre aqueles que se baseiam no sistema de equações normais, existe o operador SWEEP (veja GOODNIGHT \& SPEED, 1980), que fornece mais informações para a regressão do que o método de Cholesky.

BOLDMAN \& VAN VLECK (1991); BOLDMAN et al. (1993) e MEYER (1993) adotam a decomposição de Cholesky na obtenção de $\log |C|$ e $y^{\prime} P y$, para estimação de componentes de covariancia em análise multivariada, adotando-se o método REML. A matriz de coeficientes $C$ é decomposta no produto de matrizes triangulares inferiores $L=\left[l_{i j}\right]$ com $l_{i j}=0$ para $j>i$ e suas transpostas. Para que o método seja aplicado, os autores impõem restrições paramétricas na formação da matriz $C$, tornando-a positiva definida, consistindo na tomada de uma submatriz de $C$ de posto completo. Então, para

$$
C=\left(\begin{array}{ll}
X^{\prime} R^{-1} X & X^{\prime} R^{-1} Z \\
Z^{\prime} R^{-1} X & Z^{\prime} R^{-1} Z+G^{-1}
\end{array}\right),
$$

tem-se, após a imposição das restrições na matriz $X$, 


$$
C=L L^{\prime}=\left(\begin{array}{ll}
X^{\prime *} R^{-1} X^{*} & X^{\prime *} R^{-1} Z \\
Z^{\prime} R^{-1} X^{*} & Z^{\prime} R^{-1} Z+G^{-1}
\end{array}\right) .
$$

Como o determinante de uma matriz triangular é simplesmente o produto de seus elementos diagonais, resulta que $\log |C|=2 \sum_{i=1}^{r(C)} \log \left(l_{i i}\right)$, para $l_{i i} \neq 0$. Em seguida, através de substituições, obtêm-se as soluções para os efeitos fixos e aleatórios do modelo linear misto e $y^{\prime} P y$ é calculado como $y^{\prime} P y=y^{\prime} R^{-1} y-y^{\prime} R^{-1} X^{*} \hat{\beta}^{*}-y^{\prime} R^{-1} Z \hat{u}$.

\subsubsection{Métodos baseados na diagonalização}

\subsubsection{Eliminação de Gauss - Jordan}

O método de Gauss-Jordan é um dos mais antigos métodos de solução de sistemas de equações lineares. Além de ser um processo simples e fácil de entender, muitos métodos que possuem comportamento superior são adaptações desse método (MACHADO, 1993).

O processo desenvolve-se baseado no fato de que qualquer matriz quadrada $A$ é linha-equivalente a uma matriz diagonal, sendo obtida a partir de um número finito de operações elementares nas linhas e/ou colunas de $A$. Se $A$ é real, então a matriz resultante será também real. $\mathrm{O}$ método é similar à eliminação de Gauss, mas no método de GaussJordan os elementos abaixo e acima da diagonal da matriz resultante serão eliminados, de tal forma que a retrosubstituição não mais será necessária.

Os pivôs são escolhidos por um dos métodos sugeridos na eliminação de

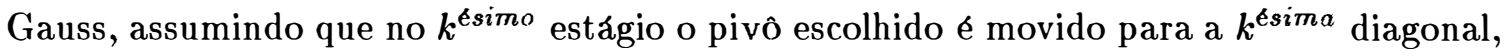
pela troca apropriada de linhas e colunas. O resultado da eliminação será:

$$
\begin{aligned}
a_{i j}^{(k)} & =a_{i j}^{(k-1)}-\left(\frac{a_{i k}^{(k-1)}}{a_{k k}^{(k-1)}}\right) a_{k j}^{(k-1)} \text { para } \mathrm{i}=1, \ldots, \mathrm{k}-1, \mathrm{k}+1, \ldots, \mathrm{n} \text { e } \mathrm{j}=\mathrm{k}, \ldots, \mathrm{n} \\
b_{i j}^{(k)} & =b_{i j}^{(k-1)}-\left(\frac{a_{i k}^{(k-1)}}{a_{k k}^{(k-1)}}\right) b_{k j}^{(k-1)} \text { para } \mathrm{i}=1, \ldots, \mathrm{k}-1, \mathrm{k}+1, \ldots, \mathrm{n} \text { e } \mathrm{j}=\mathrm{k}, \ldots, \mathrm{r} \\
a_{i j}^{(k)} & =a_{i j}^{(k-1)} \text { para } \mathrm{i}=1, \ldots, \mathrm{n} \quad \text { e } \mathrm{j}=1, \ldots, \mathrm{k}-1
\end{aligned}
$$

Se o estágio $\mathrm{k}=0$ representa as matrizes originais $A$ e $B$, a forma diagonal desejada é obtida na conclusão do estágio k $=\mathrm{n}-1$. As soluções de $A X=B$ são então dadas por:

$$
x_{i j}=\frac{b_{i j}^{(n-1)}}{a_{i i}^{(n-1)}} \text { para } \mathrm{i}=1, \ldots, \mathrm{n} \text { e } \mathrm{j}=1, \ldots, \mathrm{r}
$$

A representação $L D U$ é ignorada neste processo, uma vez que a ordem das operações reduz uma coluna completa por vez e nenhuma forma triangular superior intermediária é obtida. Como na eliminação de Gauss, todas operações realizadas nas linhas de $A$ também o são, ao mesmo tempo, realizadas nas linhas de $B$. Além disso, qualquer troca entre colunas da matriz $A$ requer a correspondente troca entre linhas de $A^{-1} B$, ou seja, o método de 
Gauss-Jordan tem por base o fato que operações elementares (troca de linhas, de colunas e substituição de uma linha por uma combinação linear dela própria com uma outra) realizadas sobre $A$ não alteram nem a solução $X$ nem a matriz $B$, desde que operações idênticas também sejam realizadas sobre essas últimas matrizes. Evidentemente, toda troca que for feita deverá ser desfeita ao final, para que as soluções correspondam à ordem inicial das incógnitas (MACHADO, 1993).

De acordo com PRESS et al. (1992) e MACHADO (1993), a eliminação de Gauss-Jordan possui dois problemas básicos: O primeiro é que todos os vetores do segundo membro do sistema de equações precisam estar disponfveis na memória e manipulados ao mesmo tempo e, segundo, a matriz de coeficientes sempre precisará ser invertida, mesmo que ela não seja necessária. Neste caso, a eliminação de Gauss-Jordan é cerca de três vezes mais lenta do que a decomposição $L U$.

\subsubsection{Decomposição por Valores Singulares (DVS)}

Dada uma matriz $A$ real e simétrica, existe uma matriz ortogonal $U$, isto é $U^{\prime} U=U U^{\prime}=I$, tal que $A=U D U^{\prime}$, e $D=U^{\prime} A U$, em que $D$ é uma matriz diagonal de autovalores de $A$. As colunas da matriz ortogonal $U$ são autovetores correspondentes aos autovalores da matriz A.

O processo de diagonalização de uma matriz real e simétrica é freqüentemente chamado de redução para a forma canônica ou decomposição por valores singulares de uma matriz simétrica. Os elementos diagonais de $D$ são denominados de valores singulares de $A$ e o número de valores singulares não nulos é igual ao posto da matriz $A$

Segundo SEARLE (1982) se $A$ é uma matriz p x q de posto r ela pode ser expressa como:

$$
A=L\left[\begin{array}{ll}
D & 0 \\
0 & 0
\end{array}\right] M^{\prime}
$$

em que $L$ e $M$ são matrizes ortogonais e $D$ é uma matriz diagonal de autovalores $\neq 0$ (positivos). De $A^{\prime} A, A A^{\prime}$ e de

$$
L^{\prime} A A^{\prime} L=\left[\begin{array}{ll}
D^{2} & 0 \\
0 & 0
\end{array}\right]_{\mathrm{p} \mathrm{x} \mathrm{p}} \quad e \quad M^{\prime} A^{\prime} A M=\left[\begin{array}{ll}
D^{2} & 0 \\
0 & 0
\end{array}\right]_{\mathrm{q} \times \mathrm{q}}, \text { tem }-s e:
$$

i) Ambos $A^{\prime} A$ e $A A^{\prime}$ são não negativas definidas e possuem os mesmos autovalores não nulos, todos positivos;

$M$ é $\mathrm{q} \times \mathrm{q}$.

ii) Nem todas matrizes de (2) têm a mesma ordem. $D^{2}$ é r x r, $L$ é p x p e

iii) As matrizes $L$ e $M$ de (2) sempre existem, mas não são únicas e nem todos pares produzem (3). No entanto, para uma escolha particular de $L$, é sempre capaz de construir $M$, tal que (2) e (3) sejam satisfeitos. (Maiores detalhes, em SEARLE, 1982; LIN, 1987; PRESS et al., 1992 e MACHADO, 1993). 


\subsubsection{Ortogonalização}

\subsubsection{Método da Ortogonalização Matricial - Método de Gram-Schmidt}

Seja $A X=B$ em que $A$ é real e não singular. Pelo método de GramSchmidt, existe uma matriz triangular não singular, digamos $L^{-1}$ que transforma $A$ em uma matriz ortogonal $R$.

Seja $L^{-1} A=R$ ou $A=L R$. Então, $L R X=B$ ou $X=R^{-1} L^{-1} B$. Desde que $R$ é ortogonal, $R R^{\prime}=D$ ou $R^{-1}=R^{\prime} D^{-1}$, e $D$ é diagonal. Por isto, $X=$ $\left(R^{\prime} D^{-1} L^{-1}\right) B$.

Note que se pode tomar $F=D^{-\frac{1}{2}} R \operatorname{com} F F^{\prime}=D^{-1 / 2} R R^{\prime} D^{1 / 2}=I$.

$\mathrm{O}$ algorftmo para encontrar $R$ é conhecido como método de Gram-Schmidt.

Seja $R=\left[\boldsymbol{r}_{i j}\right]$. Então, pelo método de Gram-Schmidt, tem-se:

$$
\begin{aligned}
r_{1 j} & =a_{1 j} \\
r_{2 j} & =a_{2 j}-l_{21} r_{1 j} \\
\vdots & =\vdots \\
r_{i j} & =a_{i j}-\left(\sum_{k=1}^{i-1} l_{i k} r_{k j}\right) \mathrm{e} \\
l_{i k} & =\sum_{j=1}^{n}\left(\frac{r_{k j} a_{i j}}{r_{k j}^{2}}\right) \quad \text { para } \mathrm{k}=1, \ldots, \mathrm{i}-1 \\
l_{i i} & =1, \quad \text { para } \mathrm{i}=1, \ldots, \mathrm{n}
\end{aligned}
$$

Existem outros métodos de ortogonalização que não serão abordados neste trabalho, mas podem ser vistos em WESTLAKE (1968).

\subsubsection{Métodos da Partição}

\subsubsection{Procedimento Padrão de Partição}

A inversa de uma matriz não singular $A$ pode ser obtida em termos de inversas de matrizes de ordens menores. Seja $A$ de ordem $\mathrm{n}$ e $\mathrm{p}+\mathrm{q}=\mathrm{n}$. Partindo A em quatro submatrizes $A_{1}, A_{2}, A_{3}$ e $A_{4}$ de dimensões p x p, p x p, q x q e q x q, respectivamente, tem-se:

$$
A=\left[\begin{array}{ll}
A_{1} & A_{2} \\
A_{3} & A_{4}
\end{array}\right]
$$

A inversa de $A$ pode ser partida similarmente como

$$
A^{-1}=\left[\begin{array}{ll}
\alpha_{1} & \alpha_{2} \\
\alpha_{3} & \alpha_{4}
\end{array}\right]
$$


Desejamos encontrar $\alpha_{i}$ em função de $A_{i}$. De $A A^{-1}=I$, tem-se:

$$
\begin{array}{ll}
A_{1} \alpha_{1}+A_{2} \alpha_{2}=I & A_{3} \alpha_{1}+A_{4} \alpha_{3}=0 \\
A_{1} \alpha_{2}+A_{2} \alpha_{4}=0 & A_{3} \alpha_{2}+A_{4} \alpha_{4}=I .
\end{array}
$$

Este sistema pode ser resolvido de diversas formas para $\alpha_{1}, \alpha_{2}, \alpha_{3}, \alpha_{4}$. A melhor formulação da solução é aquela que envolve um número mínimo de inversões matriciais. Um procedimento para resolver o sistema é :

a) Calcula-se $A_{1}^{-1}$;

b) Calcula-se $\Delta=A_{4}-\left(A_{3} A_{1}^{-1}\right) A_{2}$;

c) $\quad \alpha_{4}=\Delta^{-1}$

$$
\begin{aligned}
\alpha_{3} & =\Delta^{-1}\left(A_{3} A_{1}^{-1}\right) \\
\alpha_{2} & =-\left(A_{1}^{-1} A_{2}\right) \Delta^{-1} \\
\alpha_{1} & =\left(A_{1}^{-1}-\left(A_{1}^{-1} A_{2}\right) \alpha_{3} .\right.
\end{aligned}
$$

Sistemas de equações lineares da forma $A X=B$ podem ser resolvidos como:

$$
\begin{aligned}
& X=\left[\begin{array}{l}
X_{1} \\
X_{2}
\end{array}\right]=A^{-1} B=\left[\begin{array}{ll}
\alpha_{1} & \alpha_{2} \\
\alpha_{3} & \alpha_{4}
\end{array}\right]\left[\begin{array}{l}
B_{1} \\
B_{2}
\end{array}\right] \\
& X_{1}=\alpha_{1} B_{1}+\alpha_{2} B_{2} \quad X_{2}=\alpha_{3} B_{2}+\alpha_{4} B_{2} .
\end{aligned}
$$

Em que $B$ é de dimensão n x r, $B_{1}$ p x r e $B_{3}$ q x r. Então, $X_{1}$ tem dimensão p x r e $X_{3}$ q x r.

$$
\text { Assumindo-se } A=\left[\begin{array}{ll}
A_{11} & A_{12} \\
A_{21} & A_{22}
\end{array}\right]
$$

em que $A$ é uma matriz quadrada e simétrica e adotando-se o procedimento da partição, a inversa de $A$ pode ser obtida como:

$$
\begin{gathered}
A^{-1}=\left[\begin{array}{cc}
A_{11}+A_{11}^{-1} A_{12} A_{22.1}^{-1} A_{21} A_{11}^{-1} & -A_{11}^{-1} A_{12} A_{22.1}^{-1} \\
-A_{22.1}^{-1} A_{21} A_{11}^{-1} & A_{22.1}^{-1}
\end{array}\right] \\
\text { para } A_{22.1}=A_{22}-A_{21} A_{11}^{-1} A_{12} .
\end{gathered}
$$

Existem outros processos diretos, os quais podem ser utilizados na solução de sistemas de equações lineares. Por exemplo, existem os métodos baseados na tridiagonalização. Estes e outros não serão objeto desta discussão. 


\subsubsection{Métodos Iterativos}

Um método iterativo é uma seqüência de soluções melhoradas a partir de uma solução inicial. Os métodos iterativos são usualmente preferidos nas situações de grandes sistemas esparsos, porque eles levam em consideração os elementos nulos das matrizes, fazendo correções apropriadas para reduzir erros de arredondamento. Tais métodos são particularmente bons em sistemas quase ou totalmente diagonais, nos quais a convergência é rápida. Eles não são eficientes, no entanto, para inversão de matrizes, bem como para solução de alguns sistemas de equações lineares. A grande desvantagem dos métodos iterativos é a possibilidade de convergência lenta ou irregular. $\mathrm{O}$ último caso pode ocorrer quando a matriz $A$, do sistema $A x=b$, apresenta autovalores complexos. Convergência lenta pode ser amenizada pelo uso de procedimentos de aceleração da convergência.

Dado um sistema linear $A x=b$, segundo a notação de WESTLAKE (1968) e de PRESS et al. (1992), define-se uma itera.çáo de grau m corno uma função da forma

$$
x^{(k+1)}=F_{k}\left(A, b, x^{(k)}, x^{(k-1)}, \ldots, x^{(k-m+1)}\right),
$$

em que $x^{(k)}$ é o $n^{\text {ésimo }}$ componente do vetor obtido da $k^{\text {esima }}$ iteração. Deseja-se que $x^{(k)} \rightarrow h=A^{-1} b$ quando $k \rightarrow \infty$.

Usualmente, procura-se manter um valor de $\mathrm{k}$ menor possivel, tal que não haja grande perda de precisão e para que se reduza requerimentos de memória. O processo iterativo é chamado estacionário se $F_{k}=F \forall$ k, isto é, $F_{k}$ é independente de k. Caso contrário o processo é dito não estacionário. O processo é linear se $F_{k}$ é uma função linear de $x^{(k)}, \ldots, x^{(k-m+1)}$. Assumiremos que $\mathrm{m}=1$, para nossas discussões. Desta forma: $x^{(k+1)}=F_{k}\left(A, b, x^{(k)}\right)$. Uma iteração de primeiro grau é comumente escrita em uma das formas: $x^{(k+1)}=x^{(k)}+C^{(k)} p^{(k)}$, ou $x^{(k+1)}=q^{(k)}+T^{(k)} x^{(k)}$ onde, em geral, as matrizes $C^{(k)}$ e $T^{(k)}$ e os vetores $p^{(k)}$ e $q^{(k)}$ são funções de k, $A, b$ e $x^{(k)}$. Assume-se que $C^{(k)}$ e $T^{(k)}$ são matrizes não singulares e que $C^{(k)} \neq A^{-1}$.

Para que a solução seja confiável para resolver $A x=b$, há necessidade de que a representação acima leve a um vetor solução $h=A^{-1} b$, invariante, isto é, se $x^{(k)}=A^{-1} b$, então, deve-se obter $x^{(k+1)}=A^{-1} b \Rightarrow C^{(k)} p^{(k)}=0$ quando $x^{(k)}=A^{-1} b$ e que $\boldsymbol{q}^{(k)}=\left(I-T^{(k)} A^{-1} b\right)$.

Seja o $k^{\text {esimo }}$ vetor de residuo $r^{(k)}=b-A x^{(k)}$ e o $k^{\text {esimo }}$ vetor de erros $e^{(k)}=h-x^{(k)}$, em que $h$ é o vetor solução. Note que $r^{(k)}=A e^{(k)}$. O processo converge se e somente se $\left\|e^{(k)}\right\| \rightarrow 0$ quando $k \rightarrow \infty$. Por ser $h$ desconhecido, normalmente adota-se o comprimento do vetor de residuo $\left\|r^{(k)}\right\|$ ou o comprimento $\left\|x^{(k)}-x^{(k-1)}\right\|$ como teste de convergência.

O processo iterativo pode então ser definido como: $x^{(k+1)}=x^{(k)}+C^{(k)} r^{(k)}=$ $\left(I-C^{(k)} A\right) x^{(k)}+C^{(k)} b$ ou $x^{(k+1)}=T^{(k)} x^{(k)}+\left(I-T^{(k)}\right) A^{-1} b$, que são satisfeitos para $x^{(k)}=$ $x^{(k+1)}=A^{-1} b$ e tem-se que $A^{-1} b=\left(I-C^{(k)} A\right) A^{-1} b+C^{(k)} b$, tal que subtraindo a quantidade $x^{(k+1)}=\left(I-C^{(k)} A\right) x^{(k)}+C^{(k)} b$ resulta em $\left(A^{-1} b-x^{(k+1)}\right)=\left(I-C^{(k)} A\right)\left(A^{-1} b-x^{(k)}\right)$ ou $e^{(k+1)}=\left(I-C^{(k)} A\right) e^{(k)}$. Similarmente para a segunda aproximação, $e^{(k+1)}=T^{(k)} e^{(k)}$ de onde sucede que $e^{(k+1)}=\prod_{i=0}^{k} T^{(i)} e^{(0)}$ ou $e^{(k+1)}=\prod_{i=0}^{k}\left(I-C^{(i)} A\right) e^{(0)}$. As matrizes $T^{(k)}$ e $\left(I-C^{(k)} A\right)$ são chamadas matrizes de iteração e erros reduzidos, respectivamente. Para maiores detalhes, veja WESTLAKE (1968) e PRESS et al. (1992). 
São inúmeros os métodos iterativos, apropriados para se obter as soluçôes de sistemas de equações lineares ou não lineares. Alguns destes métodos são lineares e outros não. Apresenta-se a seguir uma descrição resumida dos métodos, apenas aqueles que são mais utilizados no processo de estimação de componentes de covariância em modelos lineares mistos, uni ou multivariados.

\subsubsection{Iteração Não Linear}

\subsection{Métodos Gradientes}

Os métodos gradientes são classificados conforme a quantidade de informação requerida da derivada da função ou do sistema. Os métodos de Newton utilizam primeira e segunda derivadas, isto significa geometricamente que estes métodos exploram a inclinação e curvatura da função a ser avaliada, apresentando convergência (quando houver) mais rápida. Outros métodos utilizam informação apenas da primeira derivada. Estes incluem, entre outros, os métodos da máxima inclinação descendente, gradiente conjugado, Cauchy, Householder e Quasi-Newton aproximando segundas derivadas. Para estimação de componentes de covariância pelo método REML, os algorítmos comumente empregados recaem nestas categorias. $\mathrm{O}$ método Escore de Fisher é um caso especial dos procedimentos de Newton, requerendo valores esperados da segunda derivada parcial da função de verossimilhança ou da matriz de informação em relação aos parâmetros a serem estimados. Como este é um procedimento em que a obtenção da segunda derivada parcial em relação aos parâmetros é geralmente diffcil, na área de melhoramento animal, na estimação de componentes de covariância, tem-se usado com maior freqüência o algoritmo EM (maximização de esperanças) (DEMPS'IER et al., 1977), o qual explora informação da primeira derivada. Este algoritmo faz uso de alguns métodos iterativos, como método de Jacobi ou Gauss Seidel ou método SOR (serão apresentados a seguir), para obtenção das soluções do sistema de equações lineares da forma $A x=b$.

\subsection{Método Simplex}

O método simplex ou método politopo, descrito por NELDER \& MEAD (1965) é um procedimento para localização do mínimo de uma função com respeito a várias variáveis. $\mathrm{O}$ método é utilizado no processo de estimação de componentes de covariancia adotando-se o método da máxima verossimilhança restrita (REML) para minimizar $-2 L_{2}$, em que $L_{2}$ é o logaritmo da função de verossimilhança restrita simplificada. Este método é as vezes chamado de método politopo para distingur-lo do método simplex, comumente utilizado em programação linear.

$\mathrm{O}$ algoritmo inicia com um conjunto de $\mathrm{p}+1$ vetores de parâmetros $t_{0}, t_{1}, \ldots$, $t_{p-1}, t_{p}$ e os correspondentes valores das funções para $-2 L_{2}$, designados como $F_{0}, F_{1}, \ldots$, $F_{p}$ e ordenados de tal forma que $F_{0} \leq F_{1} \leq \ldots \leq F_{p-1} \leq F_{p}$, em que p é o número de parâmetros a ser estimados. Os vetores de parâmetros formam os vértices do politopo ou figura geométrica de dimensão p.

Nos procedimentos de estimação de componentes de variância e covariância 
adotados neste trabalho, os $\mathrm{p}+1$ vetores de parâmetros iniciais são gerados pela multiplicação do vetor de valores iniciais ou valores preliminares por um fator igual a 1 acrescido de um valor percentual denominado "step-size". Cada vetor subseqüente é gerado pelo vetor precedente vezes o fator citado.

A cada passo do processo iterativo um novo politopo é formado pela geração de um novo ponto, substituindo o pior ponto $t_{p}$, isto é, o ponto com o maior valor de $-2 L_{2}$. Um novo ponto é gerado como uma combinação linear de pontos existentes por três operações: reflexão, expansão e contração.

$\mathrm{O}$ processo inicia com o cálculo de $t_{m}$ o centro dos melhores p vértices $t_{0}, t_{1}, \ldots, t_{p-1}$, em que

$$
t_{m}=\left(\frac{\sum_{i=0}^{p-1} t_{i}}{p}\right) \quad \text { para } \mathrm{i}=0,1,2, \ldots, \mathrm{p}-2, \mathrm{p}-1
$$

Este valor $t_{m}$ consiste do valor médio (excluindo o pior ponto) para cada parâmetro a ser estimado. Um novo ponto é então gerado por reflexão onde o pior ponto $t_{p}$, correspondente ao maior valor da função, é refletido na direção do ponto central, ou seja:

$$
t_{r}=t_{m}+\alpha\left(t_{m}-t_{p}\right)
$$

em que $\alpha(\alpha>0)$ é o coeficiente de reflexão. A função $-2 L_{2}$ é então avaliada para $t_{r}$, produzindo $F_{r}$. São possiveis três resultados:

a) $F_{0} \leq F_{r} \leq F_{p-1} \Rightarrow$ o ponto refletido é um ponto intermediário. Neste caso, $t_{p}$ é removido do simplex e $t_{r}$ é adicionado e os vetores são reordenados de tal forma que $F_{0} \leq F_{1} \leq \ldots \leq F_{p}$

b) $F_{r}<F_{0} \Rightarrow$ o ponto refletido tem o menor valor da função e é portanto o novo melhor ponto. Assume-se como boa a direção da reflexão e é feita uma tentativa para encontrar ainda um melhor ponto pela expansão do ponto refletido na mesma direção, para fornecer um ponto expandido $t_{e}$ :

$$
t_{e}=t_{m}+\gamma\left(t_{\tau}-t_{m}\right)
$$

em que $\gamma(\gamma>1)$ é o coeficiente de expansão. A função $F_{e}$ é então avaliada com dois possiveis resultados:

$\left.b_{1}\right) F_{e}<F_{r} \Rightarrow$ A expansão foi bem sucedida e $t_{e}$ substitui $t_{p}$ no final da iteração;

$\left.b_{2}\right) F_{e}>F_{p} \Rightarrow$ Falha de expansão tal que $t_{e}$ é descartado e o ponto refletido $t_{r}$ substitui $t_{p}$ para completar a iteração;

c) $F_{r}>F_{p-1} \Rightarrow$ neste caso o ponto refletido é pior do que qualquer dos p pontos iniciais. Isto indica que o politopo refletido é muito alto (grande) e deve ser contrardo. Neste caso utiliza-se uma das duas alternativas de contração. A escolha de uma delas será determinada pelos valores relativos de $F_{r}$ e $F_{p}^{\prime}$, isto é:

$\left.c_{1}\right) F_{r}<F_{p} \Rightarrow \mathrm{O}$ ponto refletido $t_{r}$ é melhor do que o ponto de máximo anterior $t_{p}$ e um ponto para contração é calculado como:

$$
t_{c}=t_{m}+\beta\left(t_{r}-t_{m}\right)
$$


em que $\beta(0<\beta<1)$ é o coeficiente de contração.

$\left.c_{2}\right) F_{r} \geq F_{p} \Rightarrow \mathrm{O}$ ponto refletido $t_{r}$ não é melhor do que o ponto de máximo $t_{p}$ anterior, e o ponto contraido $t_{c}$ é calculado como:

$$
t_{c}=t_{m}+\beta\left(t_{p}-t_{m}\right)
$$

$F_{c}$ é calculado utilizando $t_{c}$ de $c_{1}$ e $c_{2}$, sendo possfveis dois resultados:

$\left.c_{1 a}\right) F_{c}<\min \left(F_{r}^{\prime}, F_{p}\right) \Rightarrow$ A contração foi bem sucedida e $t_{c}$ substitui $t_{p}$ ao fim da iteração, ou

$\left.c_{1 b}\right) F_{c}>\min \left(F_{c}, F_{p}\right) \Rightarrow$ Houve uma falha de contração e o politopo é descartado, movendo cada um dos vetores de parâmetros $t_{1}, t_{2}, \ldots, t_{p-1}, t_{p}$ na direção da metade do melhor ponto $t_{0}$ :

$$
t_{i}=\left(t_{i}+t_{0}\right) / 2
$$

para $\mathrm{i}=1,2, \ldots$, p. A função é então avaliada para os pontos $t_{1}, \ldots, t_{p}$ e uma nova iteração é iniciada.

O politopo muda de forma a cada iteração e move em torno da função de verossimilhança até a convergência.

NELDER \& MEAD (1965) sugerem valores para $\alpha, \beta$ e $\gamma$ iguais a $1.0,0.5$ e 2.0 , respectivamente.

Desde que diferenças nos valores das funções não sejam utilizadas no estabelecimento de direção de procura, restrições no espaço de parâmetros podem ser impostas facilmente, admitindo-se um valor bem elevado para a função para um vetor de parâmetros fora do seu espaço.

O critério de convergência adotado é a variância ou desvio padrão dos valo-

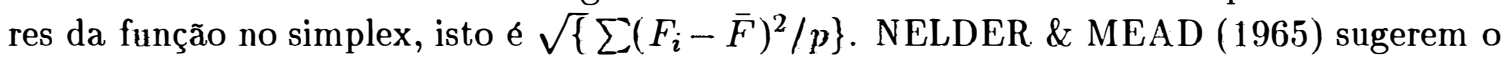
valor $<10^{-8}$. Segundo MEYER (1989), este critério é razoável, especialmente em problemas estatísticos tais como estimação por máxima verossimilhança, onde a curvatura da superf́cie próximo do minimo reflete a quantidade de informação disponfvel nos parâmetros. Todavia, se a superf́cie é plana os erros amostrais são grandes e pode ocorrer que este processo não consiga determinar o máximo da função de verossimilhança com a precisão desejável.

Para estimação de componentes de covariância, o método simplex tem provado ser robusto e fácil de se utilizar. Para pequeno número de parâmetros a ser estimados, a convergência é geralmente rápida. Para grande número de parâmetros, no entanto, especialmente em análise multivariada, o número de funções de verossimilhança avaliado pode ser grande.

Freqüentemente, as estimativas dos componentes de variância mudam pouco do caso multivariado em relação ao caso univariado. Esta observação pode ser utilizada para reduzir o número de avaliações da verossimilhança em análise multivariada. Isto pode ser feito primeiramente maximizando a verossimilhança com respeito às covariancias apenas, fixando os componentes de variância com valores obtidos no caso univariado. Posteriormente, consideram-se todos parâmetros em um segundo passo, no processo de estimação. Além disso, segundo MEYER (1993), experiência tem mostrado que o procedimento de procura normalmente requer um número excessivo de avaliações quando a 
convergência ocorre no limite do espaço de parâmetros, isto é, análise multivariada com estimativas de correlação tendendo à unidade. Nestes casos, parece vantajoso fixar tais correlações à unidade e maximizar com relação aos parâmetros remanescentes. Este método está implementado nos programas MTDFREML de BOLDMAN et al. (1993) e DFREML de MEYER (1993).

\subsection{Método das Direções Conjugadas e Método de Powell}

Métodos das direções conjugadas são baseados na geração de um conjunto de vetores ortogonais $(A)$ e então minimiza-se sucessivamente na direção de cada um deles. Um conjunto de vetores $\left(p^{k}\right), \mathrm{k}=0, \ldots, \mathrm{n}-1$ é escolhido de forma a ser conjugado (ortogonal) a $A$, isto é, $\left(A p^{(i)}\right)\left(p^{(k)}\right)=0$ para $\mathrm{i} \neq$ j. Se $A$ é positiva definida, então $\left.\left(A p^{(i)}\right)\left(p^{(j)}\right)\right)>0$. Neste caso os vetores $p^{(k)}$, linearmente independentes, são tais que qualquer solução $h$ do sistema linear $A x=b$ pode ser obtida como: $h=\sum_{i=0}^{(n-1)} \alpha_{i} p^{(i)}$. Desde que $p^{(k)}$ é ortogonal a $A$, então

$$
(A h)\left(p^{(k)}\right)=(b)\left(p^{(k)}\right)=\sum_{i=0}^{n-1} \alpha_{i}\left(A p^{(i)}\right)\left(p^{(k)}\right)=\alpha_{k}\left(A p^{(k)}\right)\left(p^{(k)}\right)
$$

$$
\text { de tal forma que } \alpha_{k}=\frac{(b)\left(p^{(k)}\right)}{\left(A p^{(k)}\right)\left(p^{(k)}\right)}
$$

Uma vez que os vetores $p^{(k)}$ foram determinados, a solução $h$ é obtida por $\mathrm{n}$ iterações da forma:

$$
\begin{aligned}
x^{(i+1)} & =x^{(i)}+\alpha_{i} p^{(i)}, \mathrm{i}=0,1, \ldots, \mathrm{n}-1 \\
\alpha_{i} & =\frac{(b)\left(p^{(i)}\right)}{\left(A p^{(i)}\right)\left(p^{(i)}\right)}, \mathrm{i}=0,1, \ldots, \mathrm{n}-1, \text { ou } \\
\alpha_{i} & =\frac{x^{(i+1)}-x^{(i)}}{p^{(i)}}
\end{aligned}
$$

iniciando com um vetor inicial $x^{(0)}=0$. Para determinar um conjunto de vetores ortogonais $y^{(k)}$ a partir de $\mathrm{n}$ vetores linearmente independentes $v^{(k)}$, usa-se, por exemplo, o procedimento de ortogonalização de Gram-Schmidt (veja (2.1.3.1)).

\section{Método de Powell}

Métodos lineares para minimizar uma função de $\mathrm{p}$ variáveis independentes geralmente utilizam uma série de minimizações unidimensionais em cada uma das $p$ direções conjugadas. Muitos métodos inicialmente procuram ao longo das direções coordenadas, mas diferem de procedimentos utilizados para gerar novas direções. No método de Powell é feita uma tentativa para gerar, após p iterações, um conjunto de $\mathrm{p}$ vetores de direções que são mutuamente conjugadas, tal que qualquer redução no valor de função 
obtida durante o processo de minimização em uma direção não é perdida quando se está fazendo a procura em uma outra direção (BOLDMAN et al., 1993). Um conjunto de direções mutuamente conjugadas minimizará uma função quadrática no processo de procura linear ao longo das direções e é muito eficiente, mesmo para funções não lineares (PRESS et al., 1992). O método de Powell também garante que os vetores de direções não se tornam linearmente dependentes porque direções dependentes encontrarão um minimo da função apenas sobre um subespaço de todo espaço p direcional. Assim, o método de Powell é tanto eficiente quanto preciso na localização de pontos de minimo em uma função.

Cada iteração do método de Powell inicia-se com um vetor de parâmetros $p_{0}^{k}$, que, como $t_{i}$ no método simplex, consiste de valores atuais para cada uma das $\mathrm{p}$ variáveis independentes e um conjunto de $\mathrm{p}$ vetores de direção $\xi_{1}^{k}, \xi_{2}^{k}, \ldots, \xi_{p}^{k}$, cada um de dimensão $\mathrm{p}$, em que $\mathrm{k}$ indica o número da iteração e o subscrito indica a direção da procura. $\mathrm{O}$ valor da função no ponto inicial $p_{0}^{k}$ é representado por $F_{0}^{k}$ e o objetivo é procurar em torno das $\mathrm{p}$ direções no sentido de se encontrar novos valores das variáveis independentes que levarão a um menor valor da função. Para cada direção $\xi_{r}^{k}$, para $\mathrm{r}=$ $1,2, \ldots, \mathrm{p}$ uma procura unidimensional é conduzida para determinar um escalar $\lambda_{r}^{k}$ tal que a função avaliada em $\left(p_{r-1}^{k}+\lambda_{r}^{k} \xi_{r}^{k}\right)$ seja um minimo ao longo da direção $\xi_{r}^{k}$ e então $p_{r}^{k}=\left(p_{r-1}^{k}+\lambda_{r}^{k} \xi_{r}^{k}\right)$ torna-se o ponto de partida para a próxima direção da iteração. Ao fim de uma iteração, após todas p direções terem sido procuradas, o ponto inicial $p_{0}^{k}$ foi movido para $p_{p}^{k}$, o qual tem o valor da função $F_{p}^{k} \operatorname{com} F_{p}^{k}<F_{0}^{k}$, isto é, próximo do minimo.

No método de Powell as direções iniciais de procura são paralelas aos eixos coordenados e a direção é modificada em cada iteração, tentando gerar um conjunto de p direções conjugadas. POWELL (1965) mostra que esta estratégia de procura é sempre eficiente e que uma função quadrática de $\mathrm{p}$ parâmetros é otimizada em $\mathrm{p}$ iterações. A convergência ocorre quando a mudança em cada variável é menor do que um critério de precisão especificado.

Algumas experiências têm mostrado que o método de Powell compara favoravelmente com o método simplex, especialmente em análise multivariada ou em análises onde se tenta separar componentes de variância com correlação amostral altamente ne-

gativa. Para vários exemplos, MEYER (1993) cita que foram necessários calcular de 30 a $50 \%$ menos valores para a função de verossimilhança quando se adotou o método de Powell. No entanto, em alguns casos o processo de procura terminou prematuramente ou não convergiu, particularmente quando o critério de precisão escolhido foi muito pequeno ou quando a convergência ocorreu no limite do espaço de parâmetros.

O método, de Powell está implementado como uma das opções dos programas do sistema DFREML de MEYER (1993), para estimação de componentes de covariância em análise uni ou multivariada.

\subsubsection{Iteração Linear}

\subsection{Método de Jacobi: iterações por deslocamentos simultaneos}

$\mathrm{O}$ método de Jacobi consiste em resolver a $i^{\text {ésima }}$ equação do sistema $A x=b$ 
para $x_{i}^{(k+1)}$, utilizando os valores $x_{j}^{(k)}, \mathrm{i} \neq \mathrm{j}$ para as variáveis remanescentes, isto é:

$$
\begin{aligned}
& x_{i}^{(k+1)}=x_{i}^{(k)}+\alpha\left(x_{i}^{(k)}-x_{i}^{(k-1)}\right)+\frac{1}{a_{i i}}\left(b_{i}-\sum_{\substack{j=1 \\
j \neq i}}^{n} a_{i j} x_{j}^{(k)}\right) \\
& \text { para } \mathrm{i}=1,2, \ldots, \mathrm{n} .
\end{aligned}
$$

$\mathrm{Na}$ forma matricial tem-se:

$$
x_{i}^{(k+1)}=x_{i}^{(k)}+\alpha\left(x_{i}^{(k)}-x_{i}^{(k-1)}\right)+D^{-1}\left(b-A x^{(k)}\right)
$$

em que $D$ é a matriz contendo os elementos diagonais de $A$, k é o número da iteração, n é a dimensão do vetor a ser resolvido e $\alpha$ é um fator de "relaxation". Obtido desta forma, o método chama-se método de Jacobi de segunda ordem. Quando $\alpha=0$ o método torna-se:

$$
x_{i}^{(k+1)}=x_{i}^{(k)}+\frac{1}{a_{i i}}\left(b_{i}-\sum_{\substack{j=1 \\ j \neq i}}^{n} a_{i j} x_{j}^{(k)}\right) .
$$

E na forma matricial

$$
x_{i}^{(k+1)}=x_{i}^{(k)}+D^{-1}\left(b-A x^{(k)}\right)
$$

que é o método de Jacobi regular.

$\mathrm{O}$ processo inicia-se fornecendo-se valores iniciais do vetor de estimativas $x^{(0)}$. Na ausência de um bom vetor de valores preliminares para $x^{(0)}$, pode-se aproximá-lo por $b_{i} / a_{i i}$.

\subsection{Método de Gauss-Seidel por deslocamentos sucessivos}

Este método é bem utilizado na prática e apresenta como uma das principais propriedades a velocidade de convergência. Esta é, em certas circunstancias, cerca de duas vezes mais rápida do que no método de Jacobi (MISZTAL \& GIANOLA, 1987). O processo iterativo pelo método de Gauss Seidel para solução do sistema $A x=b$ pode ser especificado como: abaixo:

$$
x_{i}^{(k+1)}=\frac{1}{a_{i j}}\left(b_{i}-\sum_{j=i+1}^{n} a_{i j} x_{j}^{(k)}-\sum_{j=1}^{i-1} a_{i j} x_{j}^{(k+1)}\right)
$$

para $\mathrm{i}=1,2, \ldots, \mathrm{n}$. Ou, na forma matricial,

Tomando-se $A=D+L+U$, em que $D$ é uma matriz diagonal composta de elementos diagonais de $A, L$ é uma porção diagonal inferior da matriz $A$, excluindo os elementos diagonais e $U$ é uma porção triangular superior de $A$ menos a diagonal. Então o processo iterativo pelo método de Gauss Seidel para resolver $A x=b$ é descrito por:

$$
\begin{aligned}
b & =(D+L) x^{(k+1)}+U x^{(k)} \\
x^{(k+1)} & =-(D+L)^{-1} U x^{(k)}+(D+L)^{-1} b .
\end{aligned}
$$


$\mathrm{O}$ método que é estacionário e linear, assume valores para $x_{2}^{(0)}, x_{3}^{(0)}, \ldots, x_{n}^{(0)}$ e calcula $x_{1}^{(1)}$ da primeira equação, usa $x_{1}^{(1)}, x_{2}^{(0)}, x_{3}^{(0)}, \ldots, x_{n}^{(0)}$ na segunda equação, para calcular $x_{2}^{(1)}$, e assim por diante. Em geral $x_{i}^{(k+1)}$ é calculado da $i^{\text {esima }}$ equação utilizando $x_{1}^{(k+1)}, \ldots, x_{i-1}^{(k+1)}, x_{i+1}^{(k)}, \ldots, x_{n}^{(k)}$. Não é necessário que as equações sejam utilizadas na ordem $1,2, \ldots, n$. Entretanto, a ordem tem de ser mantida durante o processo iterativo. As equações têm de ser ordenadas de tal forma que $a_{i i} \neq 0 \forall i$. Se $A$ é real e simétrica e $a_{i i}>0 \forall i$, então, o método de Gauss Seidel sempre converge quando $A$ é positiva definida (WESTLAKE, 1968). Como no método de Jacobi, na ausência de valores iniciais adequados, a aproximação $x_{i}^{(0)}=b_{i} / a_{i i}$ pode ser utilizada como valor inicial. Uma desvantagem da aplicação do método é que a convergência é sempre linear, e, quando os autovalores da matriz $A$ forem complexos a convergência é irregular. Quando os autovalores da matriz $A$ são reais e todos diferentes em valor absoluto, então, pode-se aplicar os procedimentos de aceleração da convergência, produzindo bons resultados.

MISZTAL (1993) utiliza este método na estimação de componentes de covariância, adotando-se o algorf́tmo EM-REML, para cálculo de autovalores em cada passo do processo de estimação.

\subsection{Método de Gauss-Seidel para "trás" e para "frente"}

Este processo foi desenvolvido para contornar uma deficiência do método de Gauss-Seidel quando a matriz $A$ apresenta autovalores complexos.

Por esta técnica, assumindo-se que $A$ é simétrica e definida, inicia-se o processo com um vetor aproximação $x^{(0)}$ e encontra-se $x^{(1)}$ pelo método de Gauss-Seidel regular. Obtém-se, posteriormente, $x^{(2)}$ pela aplicação do método de Gauss-Seidel para as equações em ordem reversa. Obtém-se $x^{(3)}$ por Gauss-Seidel regular, e assim sucessivamente. Para aplicação deste método, toma-se $\mathbf{j}=\mathrm{n}, \mathrm{n}-1, \ldots, 1$ e resolve-se a $j^{\text {ésima }}$ equação para $x_{j}^{(k+1)}$, utilizando $x_{p}^{(k+1)}, p>j$ obtida das $(\mathrm{n}-\mathrm{j})$ equações anteriores e $x_{p}^{(k)}, p<j$ para as variáveis remanescentes.

Por este procedimento, para $A=D+L+U$, em que $D, L$ e $U$ já foram especificados anteriormente, tem-se:

$$
x^{(k+1)}=(D+U)^{-1}\left\{L(D+L)^{-1} U x^{(k)}+\left(I-L(D+L)^{-1}\right) b\right\}
$$

\subsection{Método SOR "Successive overrelaxation"}

Este método mostra-se particularmente útil na solução de sistemas de equações lineares. Assumindo que $A$ seja simétrica e positiva definida, as soluções para $A x=b$ pelo método SOR são descritas como:

$$
\begin{gathered}
x_{i}^{(k+1)=}-\frac{\alpha}{a_{i i}}\left(\sum_{j=1}^{i-1} a_{i j} x_{j}^{(k+1)}+\sum_{j=i+1}^{n} a_{i j} x_{j}^{(k)}-b_{i}\right)-(\alpha-1) x_{i}^{(k)} \\
\text { para } \mathrm{i}=1,2, \ldots, \mathrm{n} . \quad \text { Em notação matricial, }
\end{gathered}
$$




$$
\begin{aligned}
& x_{i}^{(k+1)}=-\alpha D^{-1}\left(L x^{(k+1)}+U x^{(k)}-b\right)-(\alpha-1) I x^{(k)} o u \\
& x_{i}^{(k+1)}=\left(I-\left(\alpha^{-1}(D+L)^{-1} A\right) x^{(k)}+\left(\alpha^{-1} D+L\right)^{-1}\right) b .
\end{aligned}
$$

No caso presente, $A=D+L+U$ com $D=\left[a_{i i}\right], L=\left[a_{i j}\right], \mathrm{i}>\mathrm{j}$ e $U=\left(a_{i j}\right), \mathrm{i}<\mathrm{j}$, para $\mathrm{i}, \mathrm{j}=1,2, \ldots$, n. $x_{i}$ e $b_{i}$ são elementos de $x$ e $b$, respectivamente, $a_{i i}$ e $a_{i j}$ são elementos de $A$, k é o número da iteração e $\alpha$ é o fator de "relaxation".

Para $\alpha=1$ o método torna-se o método de Gauss-Seidel regular.

Para $0<\alpha<1$ o método é denominado "underrelaxation" $\mathrm{e}$

Para $1<\alpha<2$ o método é denominado "overrelaxation".

MISZTAL \& GIANOLA (1987) apresentam uma forma simplificada do método SOR dada por:

$$
x_{i}^{(k+1)}=x_{i}^{(k)}-\frac{\alpha}{a_{i i}}\left(\sum_{j=1}^{i-1} a_{i j} x_{j}^{(k+1)}+\sum_{j=1}^{n} a_{i j} x_{j}^{(k)}-b_{i}\right)
$$

\subsection{Procedimento Para Aceleração da Convergência: Método $\Delta^{2}$ de Ait- ken}

Algumas vezes ocorre que um processo iterativo, embora convergente, converge tão vagarosamente, a ponto de ter uma aplicação limitada. Em tais casos, algumas vezes, pode ser utilizada uma técnica de aceleração para incrementar a convergência.

Dada uma seqüência de números reais ou complexos $x_{n}$, que converge linearmente para o limite $\alpha$, o método de Aitken produz uma nova seqüência $y_{n}$, que converge mais rápido para $\alpha$.

Seja $e_{i}=\alpha-x_{i}$. A seqüência $x_{n}$ apresenta convergência geométrica se $e_{i+1}$ $=\mathrm{K} e_{i}$, para $\mathrm{K}$ uma constante, $|K|<1$. A sequiência $x_{n}$ é dita ter convergência linear se $e_{i+1}=\left(\mathrm{K}+\sigma_{i}\right) e_{i},|K|<1$ e $\sigma_{i} \rightarrow 0$ quando $i \rightarrow \infty$. A convergência geométrica é então um caso especial da convergência linear quando $\sigma_{i} \equiv 0$.

Para o caso de convergência geométrica, $\mathrm{K}$ pode ser eliminado entre relações sucessivas, obtendo-se:

$$
\alpha=\frac{x_{i+1} x_{i}-x_{i+1}^{2}}{x_{i+2}-2 x_{i+1}+x_{i}},
$$

tal que, dado três termos sucessivos da seqüência, o limite $\alpha$ pode ser calculado. Isto sugere que no caso de convergência linear, pode-se obter um aperfeiçoamento na convergência, definindo uma seqüência linear como segue. Para uma seqüência linearmente convergente $x_{i}$ com limite $\alpha$, define-se

$$
y_{i}=\frac{x_{i+1} x_{i}-x_{i+1}^{2}}{x_{i+2}-2 x_{i+1}+x_{i}},
$$

que pode ser escrita na forma

$$
y_{i}=x_{i}-\frac{\left(x_{i+1}-x_{i}\right)^{2}}{\left(x_{i+2}-2 x_{i+1}+x_{i}\right)} .
$$


ou

$$
y_{i}=x_{i}-\left(\frac{\Delta x_{i}^{2}}{\Delta^{2} x_{i}}\right) \text {. }
$$

com $\Delta x_{i}=x_{i+1}-x_{i}$ e $\Delta^{2} x_{i}=x_{i+2}-2 x_{i+1}+x_{i}$

Esta transformação é denominada método ou processo $\Delta^{2}$ de Aitken.

Se $t_{i}=\alpha-y_{i}$ e $e_{i}=\alpha-x_{i}$, então, segundo WESTLAKE (1968) e CONTE (1977), para i suficientemente grande $t_{i} / e_{i} \rightarrow 0$, tal que a sequiência $y$ converge mais rápido do que a seqüência $x$.

Existem outros procedimentos que visam tentar acelerar a convergência. Estes incluem os métodos de Chebyshev de primeiro e segundo graus, processos da soma de Euler, extrapolação $h^{2}$ de Richardson, entre outros. Para maiores detalhes, veja, por exemplo, WESTLAKE (1968), CONTE (1977) e PRESS et al. (1992) ou outros livros de análise numérica.

\subsection{Métodos de Estimação de Componentes de Covariância}

Vários são os métodos de estimação de componentes de variância e covariância. A maioria deles são invariantes à translação, isto é, independem do valor de $X \beta$, o vetor de efeitos fixos (ou seja, não são afetados por mudanças nos efeitos fixos). Alguns dos métodos são imparciais e outros são viciados. Alguns métodos requerem o conhecimento prévio dos parâmetros que se está tentando estimar e outros não o requer. Alguns métodos são iterativos e exigem maior tempo de processamento do que os métodos não iterativos. Os métodos variam em complexidade para sua implementação computacional. Os métodos diferem em magnitude da variancia amostral das estimativas (SCHAEFFER, 1993). Em geral, os métodos têm evolứdo muito, graças ao grande esforço de inúmeros pesquisadores, especialmente aqueles envolvidos com a área de estatística aplicada.

Para as deduções que serão apresentadas nesta seção, será admitido o seguinte modelo linear misto:

$$
y=X \beta+Z u+\varepsilon \stackrel{i i d}{\sim} N(X \beta, V) .
$$

Com as seguintes pressuposições:

$$
\begin{aligned}
E[u] & =E[\varepsilon]=\emptyset ; \quad E[y]=E[X \beta+Z u+\varepsilon]=X \beta ; \\
V[u] & =G ; \quad V[\varepsilon]=R ; \quad V[X \beta]=\emptyset ; \\
\Rightarrow V(y) & =V=Z G Z^{\prime}+R .
\end{aligned}
$$

Paraocasounivariado

$$
V(y)=\sum_{i=0}^{r}\left(V_{i} \sigma_{i}^{2}\right)
$$

em que r é o número de fatores aleatórios no modelo. 


\subsubsection{Métodos de Henderson}

Henderson propôs quatro métodos para estimação de componentes de variância. Os três primeiros métodos são citados na literatura como Métodos I, II e III de Henderson (HENDERSON, 1953), os quais são do tipo ANOVA, porque consistem em igualar a esperança da forma quadrática de interesse ao respectivo quadrado médio, obtido na ANOVA, e resolver a(s) esquação(ões) resultante(s). O quarto método de Henderson não apresenta uma citação padrão na literatura. Os termos Método IV de Henderson (SCHAEFFER, 1993); método simples de Henderson (KEELE et al., 1991) ou MIVQUE diagonal (HENDERSON, 1984a) são comuns na literatura.

\subsubsection{Método I de Henderson}

Este método foi derivado em 1949 na tese de $\mathrm{Ph} . \mathrm{D}$. do autor. Este método aplica-se a procedimentos de análise de variância para dados balanceados ou desbalanceados em modelos completamente aleatórios, isto e, $\mathrm{X} \beta=\mu$. Este método produz estimadores não viciados e invariantes à translação. Provavelmente, trata-se de um dos métodos mais simples de se calcular os componentes de variancia. Para se obter as estimativas dos componentes de variância por este método, basta igualar as esperanças das formas quadráticas aos respectivos quadrados médios obtidos na ANOVA.

\section{Formas quadráticas: ${ }^{1}$ :}

- $Q_{1}=I \Rightarrow y^{\prime} Q_{1} y=y^{\prime} y$ é a soma de quadrados totais

- $Q_{2}=\frac{1}{N} 11^{\prime} \Rightarrow y^{\prime} Q_{2} y=\frac{1}{N} y^{\prime} 11^{\prime} y$ é a soma de quadrados para a média

- $Q_{i+2}=Z_{i}\left(Z_{i}^{\prime} Z_{i}\right)^{-1} Z_{i}^{\prime}$, para $\mathrm{i}=1$ a s, em que s é o número de fatores aleatórios no modelo. As formas quadráticas são $y^{\prime} Z_{i}\left(Z_{i}^{\prime} Z_{i}\right)^{-1} Z_{i}^{\prime} y \Rightarrow y^{\prime} Q_{i+2} y=y^{\prime} Z_{i}\left(Z_{i}^{\prime} Z_{i}\right) Z_{i}^{\prime} y$.

Note que $Z_{i}^{\prime} Z_{i}$ é uma matriz diagonal cujos elementos são o número de observações em cada nível do $i^{\text {esimo }}$ fator aleatório e que os elementos de $Z_{i}^{\prime} y$ são as somas das observações para cada nivel do $i^{\text {esimo }}$ fator aleatório. Se $n_{i k}$ representa o $k^{\text {esimo }}$ elemento da diagonal de $Z_{i}^{\prime} Z_{i}$, então

$$
y^{\prime} Q_{i+2} y=\sum_{k=1}^{q_{i}} \frac{y_{i k}^{2}}{n_{i k}}
$$

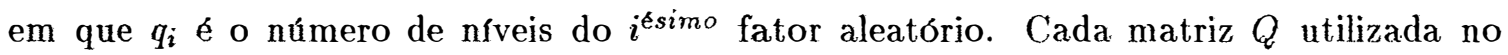
método I de Henderson tem as seguintes propriedades:

a) $Q_{m} 1=1$ para $\mathrm{m}=1 \mathrm{a}(\mathrm{s}+2)$. Cada matriz $Q$ tem elementos que adiciona 1 em cada linha (isto significa que as estimativas dos componentes de variancia serão invariantes à translação), e

\footnotetext{
${ }^{1}$ As derivações serão para o caso univariado
} 
b) $Q_{m} Q_{m}=Q_{m}$. $\Rightarrow Q_{m}$ é uma matriz idempotente (o que computacionalmente simplifica o processo de obtenção das esperanças das formas quadráticas).

\section{Esperanças das formas quadráticas:}

As esperanças das formas quadráticas são fáceis de ser obtidas. Seja o modelo apresentado em (4), (página 27). Então:

$$
\begin{aligned}
E\left[y^{\prime} Q y\right] & =\operatorname{Tr}(Q V)+\beta^{\prime} X^{\prime} Q X \beta . \text { Por este método } \\
X \beta & =1 \mu, \text { tal que } \\
\beta^{\prime} X^{\prime} Q_{1} X \beta & =\mu^{2} 1^{\prime} Q_{1} 1 . \mathrm{E} \text { como } Q_{1}=\mathrm{I}, \text { então } \\
\mu^{2} 1^{\prime} Q_{1} 1 & =\mu^{2} 1^{\prime} 1=N \mu^{2}
\end{aligned}
$$

Assim, todas formas quadráticas no método I tem $N \mu^{2}$ como parte de suas esperanças. Conseqüentemente, quando são tomadas diferenças entre estas formas quadráticas, $N \mu^{2}$ é eliminada, e, portanto, as estimativas dos componentes de variancia são invariantes à translação.

Considerando $\operatorname{Tr}(Q V)$ para cada um dos três tipos de formas quadráticas obtidas no método I, e admitindo-se que $\sigma_{0}^{2}$ representa a variancia residual, tem-se:

- $Q_{1}=I \Rightarrow y^{\prime} Q_{1} y=y^{\prime} y \Rightarrow E\left[y^{\prime} y\right]=\operatorname{Tr}(V)+N \mu^{2}$. Mas,

$$
\begin{aligned}
\operatorname{Tr}(V) & =\operatorname{Tr}\left(\sum_{i=1}^{s} Z_{i} Z_{i}^{\prime} \sigma_{i}^{2}+I \sigma_{0}^{2}\right)=\sum_{i=1}^{s}\left(\operatorname{Tr}\left(Z_{i} Z_{i}^{\prime} \sigma_{i}^{2}\right)\right)+\operatorname{Tr}\left(\operatorname{I} \sigma_{0}^{2}\right) \\
& =\sum_{i=1}^{s} \operatorname{Tr}\left(Z_{i} Z_{i}^{\prime} \sigma_{i}^{2}\right)+N \sigma_{0}^{2}=\sum_{i=1}^{s}\left(N \sigma_{i}^{2}\right)+N \sigma_{0}^{2} \\
& =\sum_{i=0}^{s} N \sigma_{i}^{2} .
\end{aligned}
$$

Observe que $\operatorname{Tr}\left(Z_{i} Z_{i}^{\prime}\right)=\operatorname{Tr}\left(Z_{i}^{\prime} Z_{i}\right)$ que é a soma de $n_{i k}$ para o $q_{i}^{\text {esimo }}$ nfvel do fator i com $\sum_{k=1}^{q_{i}} n_{i k}=N$. Portanto, a esperança de $y^{\prime} y$ no método I é sempre

$$
E\left[y^{\prime} y\right]=N \mu^{2}+\sum_{i=0}^{s} N \sigma_{i}^{2}=N \mu^{2}+N \sum_{i=0}^{s} \sigma_{i}^{2}
$$

- $Q_{2}=\frac{1}{N} \mathbf{1 1}^{\prime} \Rightarrow \operatorname{Tr}\left(Q_{2} V\right)=\sum_{i=1}^{s} \operatorname{Tr}\left(Q_{2} Z_{i} Z_{i}^{\prime} \sigma_{i}^{2}\right)+\operatorname{Tr}\left(Q_{2} \sigma_{0}^{2}\right)$

$$
=\sum_{i=1}^{s} \frac{1}{N} \sum_{k=1}^{q_{i}} n_{i k}^{2} \sigma_{i}^{2}+\sigma_{0}^{2} \cdot \text { Então }
$$

$$
E\left[\frac{1}{N} y^{\prime} 11^{\prime} y\right]=N \mu^{2}+\sum_{i=1}^{s} \frac{1}{N} \sum_{k=1}^{q_{i}} n_{i k}^{2} \sigma_{i}^{2}+\sigma_{0}^{2}
$$

Assim, a esperança da soma de quadrados, devido à média no método I, é sempre:

$$
E\left(\frac{1}{N} y^{\prime} \mathbf{1 1}^{\prime} y\right)=N \mu^{2}+\sum_{i=1}^{s} \frac{1}{N} \sum_{k=1}^{q_{i}} n_{i k}^{2} \sigma_{i}^{2}+\sigma_{0}^{2}
$$


- $Q_{m}=Z_{j}\left(Z_{j}^{\prime} Z_{j}\right)^{-1} Z_{j}^{\prime}$ para $\mathrm{m}=3 \ldots \mathrm{s}+2$ e $\mathrm{j}=1$ a $\mathrm{s}$

$$
\begin{aligned}
& \Rightarrow \operatorname{Tr}\left(Q_{m} V\right)=\sum_{i=1}^{s} \operatorname{Tr}\left(Q_{m} Z_{i} Z_{i}^{\prime} \sigma_{i}^{2}\right)+\operatorname{Tr}\left(Q_{m} \sigma_{0}^{2}\right) \\
& =\sum_{i=1}^{s} \operatorname{Tr}\left(Z_{i}^{\prime} Z_{j}\left(Z_{j}^{\prime} Z_{j}\right)^{-1} Z_{j}^{\prime} Z_{i}\right) \sigma_{i}^{2}+\operatorname{Tr}\left(Z_{j}\left(Z_{j}^{\prime} Z_{j}\right)^{-1} Z_{j}^{\prime}\right) \sigma_{0}^{2} \\
& =\sum_{i=1}^{s}\left[\sum_{k=1}^{q_{j}} \frac{\sum_{h=1}^{q_{i}} n_{k h}^{2}}{n_{k .}}\right] \sigma_{i}^{2}+\operatorname{Tr}\left(I_{q_{j}}\right) \sigma_{0}^{2}
\end{aligned}
$$

em que $n_{k h}$ é o número de observações dentro da $k h^{\text {ésima }}$ subclasse entre os fatores i e j. Se i é igual a j, então $n_{k h}=n_{k}$. e o coeficiente simplifica para N. O coeficiente de $\sigma_{0}^{2}$ simplifica para $q_{j}$.

\section{Obtenção das Estimativas}

Seja $w$ um vetor de formas quadráticas, isto é:

$$
\begin{gathered}
w^{\prime}=\left(\begin{array}{lllll}
y^{\prime} y & \frac{1}{N} y^{\prime} 11^{\prime} y & y^{\prime} Z_{1}\left(Z_{1}^{\prime} Z_{1}\right)^{-1} Z_{1} y & \cdots & y^{\prime} Z_{s}\left(Z_{s}^{\prime} Z_{s}\right)^{-1} Z_{s} y
\end{array}\right) \\
\text { e seja } \sigma^{\prime}=\left[\begin{array}{lllll}
\sigma_{0}^{2} & \sigma_{1}^{2} & \cdots & \sigma_{s}^{2}
\end{array}\right] .
\end{gathered}
$$

Então, segundo HENDERSON (1953), E[w] $=F^{\prime} \sigma+1 N \mu^{2}$. Um típico elemento de $F$ é $\operatorname{Tr}\left(Q_{j} V_{i}\right)$ para a $j^{\text {ésima }}$ forma quadrática e $i^{\text {ésimo }}$ componente. Seja $k^{\prime}$ tal que $k^{\prime} 1=0$ e $k^{\prime}$ tem posto linha completo. Então,

$$
E\left[k^{\prime} w\right]=k^{\prime} F \sigma+k^{\prime} 1 N \mu^{2}=k^{\prime} F \sigma \text { porque } k^{\prime} 1=0 .
$$

Para estimar $\sigma$, iguala-se $k^{\prime} w$ a seu valor esperado, tal que,

$$
k^{\prime} F \hat{\sigma}=k^{\prime} w
$$

ou

$$
\hat{\sigma}=\left(k^{\prime} F\right)^{-1} k^{\prime} w .
$$

Pode-se também obter as estimativas sem utilizar $k^{\prime}$. Desta forma, tem-se:

$$
\left(\begin{array}{c}
\hat{\sigma} \\
\hat{\mu}^{2}
\end{array}\right)=\left[\begin{array}{ll}
F & 1 N
\end{array}\right]^{-1} w
$$

\subsubsection{Método II de Henderson}

Tendo em vista a desvantagem do método I de Henderson, o de exigir modelos complementares aleatórios, foram desenvolvidos pelo mesmo autor os métodos II e III, os quais acomodam modelos mistos. O Método II de Henderson é um procedimento imparcial e invariante à translação, fornece estimadores únicos para os componentes de 
covariancia e é relativamente fácil de computar. Além disso, pode ser adotado em modelos mistos, desde que não haja interações entre efeitos fixos e fatores aleatórios ou aninhamentos de fator aleatório dentro de efeitos fixos. O procedimento requer as equações de mínimos quadrados para todos fatores do modelo e o vetor solução é obtido tomandose a inversa generalizada da matriz de coeficientes das equações de mínimos quadrados. Diferentes vetores solução fornecem solução única para os componentes de variancia (HENDERSON, 1984a).

\section{Derivação do Método}

- Passo 1 - Construção das equações de mínimos quadrados para todos fatores (efeitos fixos e aleatórios) do modelo

Seja

$$
\begin{aligned}
& W=\left[\begin{array}{ll}
X & Z
\end{array}\right] \text { e } \beta^{\prime}=\left[\begin{array}{ll}
\beta^{\prime} & u^{\prime}
\end{array}\right] . \text { Então } \\
& W^{\prime} W \hat{\beta}=W^{\prime} y \text { ou } \hat{\beta}=\left(W^{\prime} W\right)^{-} W^{\prime} y
\end{aligned}
$$

- Passo 2 - Ajuste de $y$ para $\hat{\beta}$, das equações de mínimos quadrados, nas equações do passo 1

$$
y^{0}=y-X \hat{\beta}
$$

- Passo 3 - Aplica-se o método I a $y^{0}$ e ajustam-se os coeficientes nas equações de todas formas quadráticas, para permitir a transformação de $V(y)$ para $V\left(y^{0}\right)$

- Passo 4 - Resolve-se para os componentes de covariância

\section{Desenvolvimento Teórico}

Seja

$$
\begin{aligned}
& W=\left[\begin{array}{ll}
X & Z
\end{array}\right]=\left[\begin{array}{llll}
X_{b} & X_{a} & Z_{a} & Z_{b}
\end{array}\right] \text { tal que }
\end{aligned}
$$

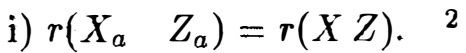

$$
\begin{aligned}
& \text { ii) } r\left(Z_{a}\right)=r(Z) \text {. } \\
& \text { Assim, } r\left(\left[\begin{array}{ll}
X & Z
\end{array}\right]\right)=r(X)+r(Z)-1 \text {. }
\end{aligned}
$$

Implica em que um menor número possivel de linhas e colunas de $Z$ são igualadas a zero, obtendo-se uma inversa generalizada da matriz de mfnimos quadrados. Então,

\footnotetext{
${ }^{2} \mathrm{r}()=$. posto $\mathrm{de}($.
} 


$$
\left(W^{\prime} W\right)=\left(\begin{array}{cccc}
X_{b}^{\prime} X_{b} & X_{b}^{\prime} X_{a} & X_{b}^{\prime} Z_{a} & X_{b}^{\prime} Z_{b} \\
X_{a}^{\prime} X_{b} & X_{a}^{\prime} X_{a} & X_{a}^{\prime} Z_{a} & X_{a}^{\prime} Z_{b} \\
Z_{a}^{\prime} X_{b} & Z_{a}^{\prime} X_{a} & Z_{a}^{\prime} Z_{a} & Z_{a}^{\prime} Z_{b} \\
Z_{b}^{\prime} X_{b} & Z_{b}^{\prime} X_{a} & Z_{b}^{\prime} Z_{a} & Z_{b}^{\prime} Z_{b}
\end{array}\right)
$$

Uma inversa generalizada de $W^{\prime} W$ é obtida (veja SEARLE, 1971) tornando-se as linhas e colunas correspondente a $X_{b}$ e $Z_{b}$ nulas e invertendo-se os elementos restantes.

$$
\operatorname{Seja}\left(W^{\prime} W\right)^{-1}=\left(\begin{array}{cccc}
0 & 0 & 0 & 0 \\
0 & P & S & 0 \\
0 & S^{\prime} & M & 0 \\
0 & 0 & 0 & 0
\end{array}\right) \Rightarrow\left(\begin{array}{cc}
P & S \\
S^{\prime} & M
\end{array}\right)\left(\begin{array}{cc}
X_{a}^{\prime} X_{a} & X_{a}^{\prime} Z_{a} \\
Z_{a}^{\prime} X_{a} & Z_{a}^{\prime} Z_{a}
\end{array}\right)=\left(\begin{array}{cc}
I & 0 \\
0^{\prime} & I
\end{array}\right)
$$

As soluções são:

$$
\left(\begin{array}{c}
\hat{\beta}_{b} \\
\hat{\beta}_{a} \\
\hat{u}_{a} \\
\hat{u}_{b}
\end{array}\right)=\left(\begin{array}{c}
0 \\
P X_{a}^{\prime} y+S Z_{a}^{\prime} y \\
S^{\prime} X_{a}^{\prime} y+M Z_{a}^{\prime} y \\
0
\end{array}\right)
$$

Um estimador nâo viciado da variância residual pode ser obtido como:

$$
\hat{\sigma}_{0}^{2}=\frac{y^{\prime} y-\hat{\beta}_{a}^{\prime} X_{a}^{\prime} y-\hat{u}_{a}^{\prime} Z_{a}^{\prime} y}{N-r(W)}
$$

Cada observação tem de ser ajustada ${ }^{3}$ para $\hat{\beta}$. Então,

$$
\begin{aligned}
y^{0} & =y-X \hat{\beta}=y-X_{b} \hat{\beta}_{b}-X_{a} \hat{\beta}_{a} \Rightarrow y-X_{a} \hat{\beta}_{a}=T y . \text { Em que } \\
T & =\left(I-X_{a} P X_{a}^{\prime}-X_{a} S Z_{a}^{\prime}\right) . \\
T Z & =\left(I-X_{a} P X_{a}^{\prime}-X_{a} S Z_{a}^{\prime}\right)\left(Z_{a} \quad Z_{b}\right)=\left(\begin{array}{ll}
Z_{a} & Z_{b}
\end{array}\right)-X_{a}\left(P X_{a}^{\prime}-S Z_{a}^{\prime}\right)\left(Z_{a} \quad Z_{b}\right) \\
& =Z-X_{a} 0=Z, \quad T X_{a}=0 \quad \text { e } \quad T X_{b}=c_{1} .(\text { SCHAEFFER, 1993) } \\
y^{0} & =T y=T X \beta+T Z u+T \varepsilon=c_{1}+Z u+T \varepsilon . \text { Consequientemente, } \\
V\left(y^{0}\right) & =V\left(c_{1}+Z u+T \varepsilon\right)=V(Z u+T \varepsilon)=\sum_{i=1}^{s} Z_{i} Z_{i}^{\prime} \sigma_{i}^{2}+T T^{\prime} \sigma_{0}^{2} .
\end{aligned}
$$

\footnotetext{
${ }^{3}$ Não necessita ser ajustada separadamente
} 
Portanto,

$$
V\left(y^{0}\right)=\sum_{i=1}^{s} Z_{i} Z_{i}^{\prime} \sigma_{i}^{2}+T T^{\prime} \sigma_{0}^{2}
$$

O coeficiente de $\sigma_{0}^{2}$ na esperança de $\frac{1}{N} y^{\prime} 11^{\prime} y$ é dado por:

$$
\begin{aligned}
(1 / N) \operatorname{Tr}\left(\mathbf{1 1}^{\prime} T T^{\prime}\right) & =(1 / N) \operatorname{Tr}\left(\mathbf{1}^{\prime} T T^{\prime} \mathbf{1}\right) \\
& =(1 / N) \operatorname{Tr}\left(\mathbf{1}^{\prime} \mathbf{1}\right)+(1 / N) \operatorname{Tr}\left(\mathbf{1}^{\prime} X_{a} P X_{a}^{\prime} \mathbf{1}\right) \\
& =1+(1 / N) \operatorname{Tr}\left(P\left(X_{a}^{\prime} \mathbf{1}\right)\left(\mathbf{1}^{\prime} X_{a}\right)\right)
\end{aligned}
$$

Assim, tem-se o coeficiente usual mais um termo adicional. Da mesma forma, o coeficiente de $\sigma_{0}^{2}$ na expressão da esperança de $y^{\prime} Z_{j}\left(Z_{j}^{\prime} Z_{j}\right)^{-1} Z_{j}^{\prime} y$, em que $Z_{j}$ é a matriz de delineamento para o $j^{\text {esimo }}$ fator aleatório do modelo, é dado por:

$$
\begin{aligned}
\operatorname{Tr}\left(Z_{j}\left(Z_{j}^{\prime} Z_{j}\right)^{-1} Z_{j}^{\prime} T T^{\prime}\right) & =\operatorname{Tr}\left(\left(Z_{j}^{\prime} Z_{j}\right)^{-1} Z_{j}^{\prime} T T^{\prime} Z_{j}\right) \\
& \left.=\operatorname{Tr}\left(\left(Z_{j}^{\prime} Z_{j}\right)^{-1}\left(Z_{j}^{\prime} Z_{j}\right)+Z_{j}^{\prime} X_{a} P X_{a}^{\prime} Z_{j}\right)\right) \\
& =\operatorname{Tr}\left(\left(Z_{j}^{\prime} Z_{j}\right)^{-1} Z_{j}^{\prime} Z_{j}\right)+\operatorname{Tr}\left(P X_{a}^{\prime} Z_{j}\left(Z_{j}^{\prime} Z_{j}\right)^{-1} Z_{j}^{\prime} X_{a}\right) \\
& =q_{j}+\operatorname{Tr}\left(P X_{a}^{\prime} Z_{j}\left(Z_{j}^{\prime} Z_{j}\right)^{-1} Z_{j}^{\prime} X_{a}\right) .
\end{aligned}
$$

Os coeficientes dos outros componentes de variancia, nas expressões dos valores esperados, são os mesmos mostrados no método I.

\subsubsection{Método III de Henderson}

O método III de Henderson é capaz de estimar componentes de variancia para qualquer tipo de modelo misto, mas computacionalmente ele requer muito mais tempo e maior capacidade de memória de computador do que os métodos I e II. Este método é comumente referido na literatura como método de ajuste de constantes de Yates, porque ele usa reduções nas somas de quadrados, devido a submodelos do modelo completo. Este método fornece estimadores não viciados e invariantes a translação, mas tais estimadores não são únicos, uma vez que se pode definir ou obter mais reduções do que as necessárias para estimar os componentes de variancia. Em alguns casos, o método fornece estimativas mais precisas dos componentes de variância do que os métodos I e II, mesmo em casos em que estes métodos são plenamente aplicáveis.

\section{Formas Quadráticas}

Para um modelo geral com s fatores aleatórios, existem $2^{s}$ possiveis submodelos e reduções que podem ser obtidas ou computadas, mas somente $\mathrm{s}+2$ reduções são necessárias para se estimar as variancias desconhecidas no caso univariado. Não existe um critério padrão para a escolha do melhor conjunto das $s+2$ reduções. Para que o método seja unicamente definido e se possa encontrar o conjunto de reduções mais preciso, SCHAEFFER (1993) apresenta as seguintes sugestões: 
- i) $y^{\prime} I y=y^{\prime} y$ é a soma de quadrados totais

- ii) Redução devido ao modelo completo: Neste caso as equações de mínimos quadrados são dadas por:

$$
\begin{aligned}
W^{\prime} W \hat{\beta} & =W^{\prime} y . \text { Então, tem-se as seguintes reduções: } \\
R\left(\beta, u_{1}, u_{2}, \ldots, u_{s}\right) & =R(\beta)=\hat{\beta}^{\prime} W^{\prime} y . \text { Assim, } \\
\hat{\sigma}_{0}^{2} & =\frac{y^{\prime} y-R(\beta)}{N-r(W)}, \text { como no método II de Henderson. }
\end{aligned}
$$

- iii) Redução devido à omissão de um fator aleatório do modelo por vez. Seja

$$
\beta_{j}=\left(\beta, u_{1}, u_{2}, \ldots, u_{j-1}, u_{j+1}, \ldots, u_{s}\right)
$$

com $u_{j}$ omitido do modelo, e seja $W_{j}$ a matriz delineamento correspondente, com $Z_{j}$ omitido. Então a redução em virtude desse submodelo, é

$$
R\left(\beta_{j}\right)=\hat{\beta}_{j}^{\prime} W_{j}^{\prime} y .
$$

Existem s reduções com um fator aleatório omitido por vez.

Para um modelo com três fatores aleatórios $\mathrm{A}, \mathrm{B}$ e $\mathrm{C}$, têm-se as seguintes reduções: $y^{\prime} y, R(\beta, A, B, C), R(\beta, A, B), R(\beta, A, C), R(\beta, B, C)$. Note que todas reduções contêm todos efeitos fixos.

Seja agora um modelo com dois fatores aleatórios com interação. Neste caso, usa-se: $y^{\prime} y, R(\beta, A, B, A B), R(\beta, A, B), R(\beta, A), R(\beta, B)$

Note que $R(\beta, A, A B)=R(\beta, B, A B)=R(\beta, A, B, A B)=R(\beta, A B)$ e, portanto, quando há interação, alguma diferença na escolha das reduções é necessária.

Suponha que existam dois fatores aleatórios no modelo e que o fator D é um efeito fixo, mas existe uma interação entre $\mathrm{D}$ e o fator A. Então, pode-se usar: $y^{\prime} y$, $R(\beta, A, B, A D), \quad R(\beta, A, B), R(\beta, A, A D), R(\beta, B)$. Para este caso

$$
R(\beta, B, A D)=R(\beta, A, B, A D) \text { e } R(\beta, A, A D)=R(\beta, A D) .
$$

\section{Valor Esperado das Reduções}

$$
\begin{aligned}
\text { Seja } C_{j} & =\left(W_{j}^{\prime} W_{j}\right)^{-} . \text {Então, } \\
\hat{\beta}_{j} & =C_{j} W_{j}^{\prime} y \mathrm{e} \\
R\left(\beta_{j}\right) & =\hat{\beta}_{j}^{\prime} W_{j}^{\prime} y=y^{\prime} W_{j} C_{j} W_{j}^{\prime} y . \quad \text { Assim, } \\
E\left(R\left(\beta_{j}\right)\right) & =\beta^{\prime} X^{\prime} W_{j} C_{j} W_{j}^{\prime} X \beta+\sum_{i=0}^{s} \operatorname{Tr}\left(W_{j} C_{j} W_{j}^{\prime} V_{i}\right) \sigma_{i}^{2} .
\end{aligned}
$$

Mas, da teoria de inversa generalizada, sabe-se que $W_{j} C_{j} W_{j}^{\prime} W_{j}=W_{j}$ (SCHAEFFER, 1993) e, consequientemente, $\beta^{\prime} X^{\prime} W_{j} C_{j} W_{j}^{\prime} X \beta=\beta^{\prime} X^{\prime} X \beta$ e $\operatorname{Tr}\left(W_{j} C_{j} W_{j}^{\prime} Z_{i} Z_{i}^{\prime}\right)=\operatorname{Tr}\left(Z_{i} Z_{i}^{\prime}\right)$ 
se $Z_{i} \subset W_{j}$ ou $\operatorname{Tr}\left(W_{j} C_{j} W_{j}^{\prime} Z_{i} Z_{i}^{\prime}\right)=\operatorname{Tr}\left(C_{j}\left(W_{j}^{\prime} Z_{i}\right)\left(Z_{i}^{\prime} W_{j}\right)\right)$ se $Z_{i} \not \subset W_{j}$. Também o coeficiente de $\sigma_{0}^{2}$ é $\operatorname{Tr}\left(W_{j} C_{j} W_{j}^{\prime}\right)=\operatorname{Tr}\left(C_{j} W_{j}^{\prime} W_{j}\right)=r\left(W_{j}\right)$, porque $C_{j} W_{j}^{\prime} W_{j}$ é idempotente. Portanto,

$$
E\left(R\left(\beta_{j}\right)\right)=\beta^{\prime} X^{\prime} X \beta+r\left(W_{j}\right) \sigma_{0}^{2}+\sum_{i=1}^{s}\left(\operatorname{Tr}\left(C_{j}\left(W_{j}^{\prime} Z_{i}\right)\left(Z_{i}^{\prime} W_{j}\right)\right)\right) \sigma_{i}^{2}
$$

Segundo SORENSEN \& KENNEDY (1984), quando ocorrem mudanças nas freqüências gênicas, quer ao acaso, em pequenas populações, ou em grandes populações, em função da seleção, os métodos I, II e III de Henderson produzem estimativas viciadas para os componentes de variância.

\subsubsection{Método IV de Henderson}

Estimação de componentes de variancia e de covariância pelos métodos ML, MIVQUE e REML (serão apresentados posteriormente), para grandes conjuntos de dados, requerem a inversão da matriz de coeficientes de grande dimensão. Se não houver um método indireto, para contornar a situação (este método foi implementado a partir de 1986), o processo de estimação se torna proibitivo. Assim, HENDERSON (1984a) derivou um método que propoe o uso de uma inversa aproximada da matriz de coeficientes.

A base para o método IV é a absorção dos efeitos fixos nas equações de minimos quadrados, para os fatores aleatórios. A absorção essencialmente ajusta o lado direito das equações, correspondentes aos efeitos aleatórios, pelos efeitos fixos e, conseqüentemente, qualquer forma quadrática envolvendo um efeito absorvido torna-se invariante à translação.

Sejam as equações de minimos quadráticos de um modelo linear misto repre-

sentado por:

$$
\left(\begin{array}{cc}
X^{\prime} X & X^{\prime} Z \\
Z^{\prime} X & Z^{\prime} Z
\end{array}\right)\left(\begin{array}{l}
\hat{\beta} \\
\hat{u}
\end{array}\right)=\left(\begin{array}{c}
X^{\prime} y \\
Z^{\prime} y
\end{array}\right)
$$

Sejam tamb́em $M=I-X\left(X^{\prime} X\right)^{-} X^{\prime}$ e a partição de $Z$ como $\left[Z_{1} Z_{2} \ldots Z_{s}\right]$. Então, as equações, após absorção dos efeitos fixos nos efeitos aleatórios, tornam-se:

$$
\left(\begin{array}{cccc}
Z_{1}^{\prime} M Z_{1} & Z_{1}^{\prime} M Z_{2} & \cdots & Z_{1} M Z_{s} \\
Z_{2}^{\prime} M Z_{1} & Z_{2}^{\prime} M Z_{2} & \cdots & Z_{2} M Z_{s} \\
\vdots & \vdots & \vdots & \vdots \\
Z_{s}^{\prime} M Z_{1} & Z_{s}^{\prime} M Z_{2} & \cdots & Z_{s} M Z_{s}
\end{array}\right) \cdot\left(\begin{array}{c}
\hat{u}_{1} \\
\hat{u}_{2} \\
\vdots \\
\hat{u}_{s}
\end{array}\right) \cdot=\left(\begin{array}{c}
Z_{1}^{\prime} M y \\
Z_{2}^{\prime} M y \\
\vdots \\
Z_{s}^{\prime} M y
\end{array}\right) .
$$

\section{Formas quadráticas após absorção das equações}

Seja $r_{j}=Z_{j}^{\prime} M y$. Então $E\left(r_{j}\right)=Z_{j}^{\prime} M E(y)=Z_{j}^{\prime} M X \beta$. mas $M X=\emptyset \mathrm{e}$ então $E\left[r_{j}\right]=\emptyset$, para $j=1,2, \cdots, s$. A matriz de variância e covariância de $Z^{\prime} M y$ é 
dada por:

$$
\begin{aligned}
V\left(Z^{\prime} M y\right) & =\sum_{i=0}^{s} Z^{\prime} M V_{i} M Z \sigma_{i}^{2} \\
& =\sum_{i=1}^{s}\left(Z^{\prime} M Z_{i}\right)\left(Z_{i}^{\prime} M Z\right) \sigma_{i}^{2}+Z^{\prime} M Z \sigma_{0}^{2} .
\end{aligned}
$$

em que $V_{i}=Z_{i}^{\prime} Z_{i}$. Seja $r^{\prime}=\left[\begin{array}{llll}r_{1}^{\prime} & r_{2}^{\prime} \ldots & r_{s}^{\prime}\end{array}\right]$, então, a forma quadrática de $r$ tem valor esperado:

$$
E\left(r^{\prime} Q r\right)=\sum_{i=1}^{s} \operatorname{Tr}\left(Q\left(Z^{\prime} M Z_{i}\right)\left(Z_{i}^{\prime} M Z\right)\right) \sigma_{i}^{2}+\operatorname{Tr}\left(Q Z^{\prime} M Z\right) \sigma_{0}^{2}
$$

Formas quadráticas utilizando diagonais das equações de mfnimos quadrados

Define-se $Q$ como uma matriz diagonal com elementos correspondendo à $\operatorname{diag}\left(Z_{j}^{\prime} Z_{j}\right)^{-1}$ e os demais elementos nulos.

Obtém-se $Q_{j}, r^{\prime} Q_{j} r, E\left(r^{\prime} Q_{j} r\right)$. Monta-se um sistema de equações em função dos componentes a serem estimados e a solução destas equações fornecerá as estimativas dos componentes desconhecidos.

\section{Derivação do Método IV}

$\mathrm{O}$ método IV utiliza conjuntos especificos de formas quadráticas, tais como: $Q_{j}=\operatorname{diag}\left(Z_{j}^{\prime} M Z_{j}+I K_{j}\right)^{-2}$ e zeros para as linhas correspondentes a outros fatores aleatórios, em que $K_{j}$ é um valor preliminar de $\sigma_{0}^{2} / \sigma_{j}^{2}$. Calcula-se $r^{\prime} Q_{j} r$ e $E\left[r^{\prime} Q_{j} r\right]$ para todos fatores aleatórios e $E\left(y^{\prime} M y\right)$. Monta-se um sistema de equações em função dos componentes a serem estimados e o resolve. Para maiores detalhes, veja HENDERSON (1984a)

\subsubsection{Os Métodos MINQUE e MIVQUE}

No final da década de 60 vários estatísticos começaram a trabalhar na obtenção de métodos de estimação de componentes de variancia que resultariam em estimadores com outras propriedades, além de imparcialidade e invariancia à translação. Sabe-se que para delineamentos balanceados, isto é, igual número de observações em todas subclasses e sem covariáveis, igualando-se os quadrados médios obtidos na ANOVA aos respectivos valores esperados, obtêm-se os estimadores quadráticos, invariantes à translação, imparciais, com minima variancia amostral (HENDERS ON, 1984a). Infelizmente, tais estimadores não podem ser derivados para o caso não balanceado, a menos que se conheça $G$ e $R$, as matrizes de variancia e covariancia dos fatores aleatórios e do erro, respectivamente, ao menos proporcionalmente.

É possivel, no entanto, derivar estimadores quadráticos, localmente melhores, invariantes à translação e imparciais, sob a pressuposição de multinormalidade. Este método é denominado MIVQUE ("Minimum Variance Quadratic Unbiased Estimation") 
e é devido a Rao (RAO, 1971a). Pioneirismo adicional em trabalhos nesta área deve-se a LaMotte (LaMOTTE, 1970) e Townsend \& Searle (TOWNSEND \& SEARLE, 1971). Os trabalhos destes pesquisadores, embora brilhantes sob o aspecto teórico, pecaram pela falta de exemplos de aplicação em dados reais(SCHAEFFER, 1993). HENDERSON (1973 e 1984a) derivou fórmulas para MIVQUE, baseadas em equações de um modelo misto e mostrou que elas eram idênticas às equações descritas por LaMotte.

O método MIVQUE se baseia na estimação de funções quadráticas das observações e usa a restrição de que a matriz núcleo seja determinada, de forma que os estimadores tenham variância minima (RAO, 1971a, SEARLE, 1979 e HENDERSON, 1984a).

\section{Formas quadraticas}

Assuma que $y$ tenha distribuição multinormal com vetor de médias $X \boldsymbol{\beta}$ e matriz de variancias e covariancias V. Seja $\theta_{i}$ as informações preliminares de $\sigma_{i}^{2}$, para $\mathrm{i}=$ 0 a s. Seja, também, $\mathrm{H}$ os valores preliminares de $\mathrm{V}$, os quais são: $H=\sum_{j=1}^{s} Z_{j} Z_{j}^{\prime} \theta_{j}+I \theta_{0}$. Uma matriz projeção é constrứda como:

$$
P=H^{-1}-H^{-1} X\left(X^{\prime} H^{-1} X\right)^{-} X^{\prime} H^{-1} \text {. Esta matriz projeção é simplesmen- }
$$

te uma maneira de absorver os efeitos fixos e ao mesmo tempo considerar as informações a respeito de $\mathrm{V}$. Note que $P X=\emptyset$ e que $P H$ é idempotente. As formas quadráticas para obtenção de MIVQUE são: $y^{\prime} P Z_{i} Z_{i}^{\prime} P y$ para $\mathrm{i}=1$ a s e $y^{\prime} P P y$. Note que estas são formas quadraticas de $P y$ e desde que $E(P y)=\emptyset$, então as formas quadráticas são invariantes a translação. Se as formas quadráticas forem reunidas, tem-se:

$$
\sum_{i=1}^{s} y^{\prime} P Z_{i} Z_{i}^{\prime} P y \theta_{i}+y^{\prime} P P y \theta_{0}=y^{\prime} P H P y=y^{\prime} P y
$$

Para simplificar estas formas quadráticas, note que:

$$
\begin{aligned}
P y & =\left(H^{-1}-H^{-1} X\left(X^{\prime} H^{-1} X\right)^{-} X^{\prime} H^{-1}\right) y \\
& =H^{-1}\left(y-X\left(X^{\prime} H^{-1} X\right)^{-} X^{\prime} H^{-1} y\right) \\
& =H^{-1}(y-X \hat{\beta}) \text { para } \\
\hat{\beta} & =\left(X^{\prime} H^{-1} X\right)^{-} X^{\prime} H^{-1} y . \\
\text { Portanto, } y^{\prime} P Z_{i} Z_{i}^{\prime} P y & =(y-X \hat{\beta})^{\prime} H^{-1} Z_{i} Z_{i}^{\prime} H^{-1}(y-X \hat{\beta}) . \\
\text { Se } \hat{u}_{i} & =G_{i} Z_{i}^{\prime} P y
\end{aligned}
$$

em que $G_{i}$ é a matriz de variancia e covariância de $\hat{u}_{i}$ (HENDERSON, 1984b), então

$$
\begin{aligned}
y^{\prime} P Z_{i} Z_{i}^{\prime} P y & =y^{\prime} P Z_{i}\left(G_{i} G_{i}^{-1} G_{i}^{-1} G_{i}\right) Z_{i}^{\prime} P y \\
& =\left(y^{\prime} P Z_{i} G_{i}\right) G_{i}^{-2}\left(G_{i} Z_{i}^{\prime} P y\right)=\hat{u}_{i}^{\prime} G_{i}^{-2} \hat{u}_{i} \\
& =\frac{\hat{u}_{i}^{\prime} \hat{u}_{i}}{\theta_{i}^{2}} \cdot \operatorname{Para} G_{i}=I \theta_{i}
\end{aligned}
$$

De forma similar, $y^{\prime} P P y=\left(y^{\prime} y-\hat{\beta}^{\prime} X^{\prime} y-\sum_{i=1}^{s} \hat{u}_{i} Z_{i}^{\prime} y-\sum_{i=1}^{s} \hat{u}_{i}^{\prime} G_{i}^{-1} \hat{u}_{i}\right)$. 
Assim, sob o modelo linear básico para estimação dos componentes de variância, as formas quadráticas necessárias para obtenção dos estimadores pelo método MIVQUE são:

$$
\hat{u}_{i}^{\prime} \hat{u}_{i} \text { e } y^{\prime} y-\hat{\beta}^{\prime} X^{\prime} y-\sum_{i=1}^{s} \hat{u}_{i}^{\prime} Z_{i}^{\prime} y
$$

\section{Valor esperado das formas quadráticas}

As equações do modelo misto são dadas por:

$$
\left(\begin{array}{lllll}
X^{\prime} X & X^{\prime} Z_{1} & X^{\prime} Z_{2} & \cdots & X^{\prime} Z_{s} \\
Z_{1}^{\prime} X & Z_{1}^{\prime} Z_{1}+I \alpha_{1} & Z_{1}^{\prime} Z_{2} & \cdots & Z_{1}^{\prime} Z_{s} \\
Z_{2}^{\prime} X & Z_{1}^{\prime} Z_{2} & Z_{2}^{\prime} Z_{2}+I \alpha_{2} & \cdots & Z_{2}^{\prime} Z_{s} \\
\vdots & \vdots & \vdots & \vdots & \vdots \\
Z_{s}^{\prime} X & Z_{1}^{\prime} Z_{s} & Z_{2}^{\prime} Z_{s} & \cdots & Z_{s}^{\prime} Z_{s}+I \alpha_{s}
\end{array}\right)\left(\begin{array}{l}
\hat{\beta} \\
\hat{u}_{1} \\
\hat{u}_{2} \\
\vdots \\
\hat{u}_{s}
\end{array}\right)=\left(\begin{array}{l}
X^{\prime} y \\
Z_{1}^{\prime} y \\
Z_{2}^{\prime} y \\
\vdots \\
Z_{s}^{\prime} y
\end{array}\right)
$$

em que $\alpha_{i}=\theta_{0} / \theta_{i}=G_{i}^{-1} G_{0}$. Seja uma inversa generalizada da matriz de coeficientes representada por:

$$
C^{-}=\left(\begin{array}{ccccc}
c^{x x} & c^{x 1} & c^{x 2} & \cdots & c^{x s} \\
c^{1 x} & c^{11} & c^{12} & \cdots & c^{1 s} \\
c^{2 x} & c^{21} & c^{22} & \cdots & c^{2 s} \\
\vdots & \vdots & \vdots & \vdots & \vdots \\
c^{s x} & c^{s 1} & c^{s 2} & \cdots & c^{s s}
\end{array}\right)=\left(\begin{array}{c}
c^{x} \\
c^{1} \\
c^{2} \\
\vdots \\
c^{s}
\end{array}\right)
$$

Sejam

$$
r=W^{\prime} y=\left(\begin{array}{llll}
y^{\prime} X & y^{\prime} Z_{1} & y^{\prime} Z_{2} \cdots y^{\prime} Z_{s}
\end{array}\right)^{\prime}
$$

o lado direito das equaç̃es normais do modelo misto e

$$
\begin{aligned}
\hat{u}_{i}=c^{i} r \Rightarrow \hat{u}_{i}^{\prime} \hat{u}_{i} & =r^{\prime} c^{i} c^{i} r \text { Então, } \\
V(r)=V\left(W^{\prime} y\right)=W^{\prime} V(y) W & =\sum_{j=1}^{s} W^{\prime} Z_{j} Z_{j}^{\prime} W \sigma_{j}^{2}+W^{\prime} W \sigma_{0}^{2} \\
& =\sum_{j=0}^{s} B_{j} \sigma_{j}^{2} \\
\text { Também } \hat{u}_{i} & =c^{i} r \text { e } \\
\hat{u}_{i}^{\prime} \hat{u}_{i} & =r^{\prime} c^{i} c^{i} r \text { e } \\
E\left(\hat{u}_{i}^{\prime} \hat{u}_{i}\right) & =\sum_{j=0}^{s} \operatorname{Tr}\left(c^{i} c^{i} B_{j}\right) \sigma_{j}^{2}
\end{aligned}
$$


Estes valores esperados podem ser simplificados (veja SCHAEFFER, 1993), especialmente considerando que $X$ é de posto coluna completo, após imposição de restrições apropriadas aos parâmetros ou às soluções. Desta forma,

$$
C^{-} W^{\prime} W=\left(\begin{array}{ccccc}
I_{x} & -c^{x 1} \alpha_{1} & -c^{x 2} \alpha_{2} & \cdots & -c^{x s} \alpha_{s} \\
0 & I-c^{11} \alpha_{1} & -c^{12} \alpha_{2} & \cdots & -c^{1 s} \alpha_{s} \\
0 & -c^{21} \alpha_{1} & I-c^{22} \alpha_{2} & \cdots & -c^{s s} \alpha_{s} \\
\vdots & \vdots & \vdots & \vdots & \vdots \\
0 & -c^{s 1} \alpha_{1} & -c^{s 2} \alpha_{2} & \cdots & I-c^{s s} \alpha_{s}
\end{array}\right)
$$

Note que $C$ depende de valores prévios de $\alpha_{i}=\theta_{0} / \theta_{i}$. Para $i \neq j$ tem-se, segundo SCHAEFFER, (1993):

$$
\begin{aligned}
\operatorname{Tr}\left(c^{i} c^{j} B_{j}\right) & =\operatorname{Tr}\left(c^{i} c^{i} W^{\prime} Z_{j} Z_{j}^{\prime} W\right)=\operatorname{Tr}\left(\left(Z_{j}^{\prime} W c^{i i}\right)\left(c^{i} W^{\prime} Z_{j}\right)\right) \\
& =\operatorname{Tr}\left[\left(-c^{i j j} \alpha_{j}\right)\left(-c^{i j} \alpha_{j}\right)\right]=\operatorname{Tr}\left(c^{j i} c^{i j}\right) \alpha_{j}^{2}
\end{aligned}
$$

e o valor esperado de $\hat{u}_{i}^{\prime} \hat{u}_{i}$ pode então ser expresso como:

$$
\begin{aligned}
E\left(\hat{u}_{i}^{\prime} \hat{u}_{i}\right) & =\left(q_{i}-2 \operatorname{Tr}\left(c^{i i} \alpha_{i}\right)+\operatorname{Tr}\left(\left(c^{i i}\right)^{2} \alpha_{i}^{2}\right)\right) \sigma_{i}^{2}+\sum_{j \neq i}^{s} \operatorname{Tr}\left(c^{i j} c^{i j} \alpha_{j}^{2}\right) \sigma_{j}^{2} \\
& +\left(\operatorname{Tr}\left(c^{i i}\right)-\operatorname{Tr}\left(\left(c^{i i}\right)^{2} \alpha_{i}\right)-\sum_{j \neq i}^{s} \operatorname{Tr}\left(c^{i j} c^{i j} \alpha_{j}^{0}\right)\right) \sigma_{0}^{2}
\end{aligned}
$$

Da mesma forma, sabe-se que

$$
\begin{aligned}
E\left(y^{\prime} y\right) & =\beta^{\prime} X^{\prime} X \beta+N \sum_{j \neq 0}^{s} \sigma_{j}^{2} \text { e, assim, } \\
r^{\prime} C^{-} r & =\hat{\beta}^{\prime} X^{\prime} y+\sum_{i=1}^{s} \hat{u}_{i}^{\prime} Z_{i}^{\prime} y . \text { Conseqüentemente, } \\
E\left(r^{\prime} C^{-} r\right) & =\beta^{\prime} X^{\prime} W C^{-} W^{\prime} X \beta+\sum_{j=0}^{s} \operatorname{Tr}\left(C^{-} B_{j} \sigma_{j}^{2}\right) . \text { Mas, } \\
X^{\prime} W C^{-} W^{\prime} X & =X^{\prime} X(\operatorname{SCHAEFFER,~1993).~Então,~} \\
E\left(y^{\prime} y-r^{\prime} C^{-} r\right) & =\sum_{j=0}^{s}\left(N-\operatorname{Tr}\left(C^{-} B_{j}\right) \sigma_{j}^{2}\right. \text { que reduz a } \\
E\left(y^{\prime} y-r^{\prime} C^{-} r\right) & =\sum_{j=1}^{s}\left(q_{j} \alpha_{j}-\operatorname{Tr}\left(c^{j j} \alpha_{j}^{2}\right) \sigma_{j}^{2}+\left(N-r(X)-\sum_{i=1}^{s}\left(q_{i}-\operatorname{Tr}\left(c^{i i} \alpha_{i}\right)\right) \sigma_{0}^{2}\right.\right.
\end{aligned}
$$

Portanto, para se obter as estimativas de $\sigma_{i}^{2}$, basta calcular:

$$
\begin{gathered}
\operatorname{Tr}\left(c^{i} c^{i} B_{i} \sigma_{i}^{2}\right), \text { para } B_{i}=W^{\prime} Z_{i} Z_{i}^{\prime} W \\
N-\operatorname{Tr}\left(C^{-} B_{i}\right) \sigma_{i}^{2}+\left(N-\operatorname{Tr}\left(C^{-} B_{0}\right)\right) \\
\hat{u}^{\prime} \hat{u} \quad \mathrm{e}
\end{gathered}
$$




$$
y^{\prime} y-r^{\prime} C^{-r}
$$

e montar o sistema de equações, de ordem igual ao número de equações, e resolvê-lo. Observe, entretanto, que MIVQUE requer os elementos inversos das equações do modelo linear misto para obtenção dos valores esperados das formas quadráticas. Isto, muitas vezes dificulta sobremaneira ou até impossibilita o uso do método, quando a ordem das equações é elevada.

RAO (1971b) também derivou o método MINQUE ou método da estimação não viesada de norma minima ("Minimum Norm Quadratic Unbiased Estimation") para o caso em que $y$ segue qualquer distribuição. Este método baseia-se na estimação de funções quadráticas dos componentes de variância, por meio de formas quadráticas das observações, com a restrição de que a norma Euclideana da matriz núcleo seja mínima.

BROWN (1976) tomou as equações derivadas de MINQUE e repetiu o processo de estimação utilizando as estimativas dos componentes obtidos no passo anterior. O procedimento foi denominado de MINQUE iterativo ou I-MIVQUE, e os estimadores derivados são assintoticamente normais.

Os dois métodos MIVQUE e MINQUE assumem que $V$, a matriz de covariância de $y$ é conhecida.

A variância da forma quadrática no caso do método MIVQUE ou a norma Euclideana da matriz núcleo no caso do método MINQUE só serão minimizadas se o valor preliminar de $V$ for o verdadeiro valor na população. Neste caso, os métodos produzem estimadores imparciais, invariantes à translação e de variancia ou norma mínima, respectivamente para MIVQUE e MINQUE.

Desde que $V$ em realidade não é conhecida, pois o que se pretende é estimála, em geral, os estimadores MIVQUE e MINQUE não são de variância ou norma minima, mas são tão bons estimadores quanto mais próximo $V$ inicial for do verdadeiro $V$ (SCHAEFFER, 1993).

Sob normalidade, os estimadores obtidos pelos dois métodos são equivalentes. Assim, o método MIVQUE é o mais comumente utilizado e conhecido, tendo em vista que na maioria dos trabalhos cientificos, na área biológica, a multinormalidade é assumida. Por esta razão não será deduzida a derivação dos componentes de variância adotando-se o método MINQUE.

\subsubsection{Método da Máxima Verossimilhança - ML}

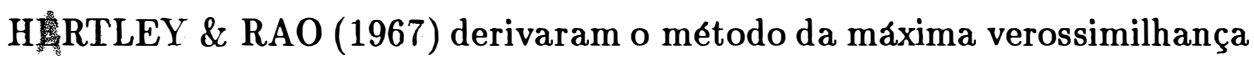
para estimação de componentes de variância.

O conceito verossimilhança consiste de uma ferramenta geral e flexivel para estimação em estatística e áreas afins. Estimação por máxima verossimilhança - ML ("Maximum Likelihood") é conceitualmente simples: para um dado modelo de análise, parâmetros a serem estimados e conhecida a distribuição dos dados, obtém-se a função de verossimilhança representada como $\Lambda$ ou função densidade de probabilidade conjunta das observações (para o caso contínuo). As estimativas de máxima verossimilhança para um conjunto especifico de dados são simplesmente os valores numéricos dos parâmetros para 
os quais $\Lambda$ alcança seu máximo. Os estimadores correspondentes são denominados estimadores de máxima verossimilhança. Na prática, em geral, por facilidades operacionais ou computacionais, maximiza-se o logaritmo da função de verossimilhança $(\Lambda)$, freqüentemente referida como função suporte (MEYER, 1993).

HARVILLE (1977) fez uma ampla revisão a respeito da estimação de componentes de variância adotando-se o método da máxima verossimilhança (ML). ML apresenta características atrativas para estimar componentes de covariancia.

Os estimadores de ML são funções de estatísticas suficientes e são consistentes e assintoticamente normais e eficientes. Sob certas condições, os estimadores ML podem ser adotados mesmo em dados amostrais não aleatórios (MEYER, 1993). Certas deficiências de outros métodos não são comuns no método ML.

Pelo método da ML, restrições para não negatividade, nos componentes de variância ou dos autovalores das matrizes de covariância, ou ainda outras restrições, no espaço paramétrico, não causam dificuldades conceituais na aplicação do método.

HOCKING \& KUTNER (1975) citam que para algum delineamento não balanceado, existem estimadores na classe dos estimadores quadráticos, não viciados, invariantes à translação, que têm variancia uniformemente menor do que os estimadores obtidos pelos métodos de Henderson. Estes estimadores são iguais ou similares àqueles obtidos pelo método da máxima verossimilhança. Em virtude destas e outras propriedades, interesse pelo uso do método da máxima verossimilhança tem crescido nos últimos anos.

\subsubsection{Derivação dos componentes de variáncia pelo método $\mathrm{ML}$}

Assumindo que $y$ seja normalmente distriburdo, com vetor de média $X \beta$ e matriz de covariância $V$ e admitindo-se o modelo apresentado em (4), (pág. 27), a função de verossimilhança de $y$ é dada por:

$$
\begin{array}{r}
\Lambda=\frac{1}{(2 \pi)^{0.5 N}|V|^{0.5}} e^{-0.5(y-X \beta)^{\prime} V^{-1}(y-X \beta)} \\
-\infty<y_{i}<\infty,-\infty<\beta_{k}<\infty \text { e } V \text { é Positiva Definida }
\end{array}
$$

O logarítmo de $\Lambda$ é dado por:

$$
\log (\Lambda)=\lambda=-0.5 N \log (2 \pi)-0.5 \log |V|-0.5(y-X \beta)^{\prime} V^{-1}(y-X \beta) .
$$

Derivando estas equações em relação aos parâmetros desconhecidos, tem-se:

$$
\begin{gathered}
\frac{\partial \lambda}{\partial \beta}=X^{\prime} V^{-1} X \beta+X^{\prime} V^{-1} y \\
\frac{\partial^{2} \lambda}{\partial \sigma_{i} \partial \sigma_{j}}=-0.5 \operatorname{Tr}\left(V^{-1} V_{i j}\right)+0.5 y V^{-1} V_{i j} V^{-1}(y-X \beta)+0.5 \beta^{\prime} X^{\prime} V^{-1} V_{i j} V^{-1} X \beta \\
\frac{\partial \lambda}{\partial \sigma_{i}^{2}}=-0.5 \operatorname{Tr}\left(V^{-1} V_{i}\right)+0.5(y-X \beta)^{\prime} V^{-1} V_{i} V^{-1}(y-X \beta) \\
\text { em que } V_{i}=\frac{\partial V}{\partial \sigma_{i}^{2}}
\end{gathered}
$$




$$
\text { e } V_{i j}=\frac{\partial^{2} V}{\partial \sigma_{i} \partial \sigma_{j}}
$$

Igualando as derivadas parciais de primeira ordem a zero, resulta:

$$
\begin{gathered}
\hat{\beta}=\left(X^{\prime} V^{-1} X\right)^{-} X^{\prime} V^{-1} y \text { e } \\
\operatorname{Tr}\left(V^{-1} V_{i}\right)=(y-X \hat{\beta})^{\prime} V^{-1} V_{i} V^{-1}(y-X \hat{\beta}) .
\end{gathered}
$$

Mas como $P y=V^{-1}(y-X \hat{\beta})$ (SCHAEFFER, 1993), em que $P$ é uma matriz projeção e $V_{i}=Z_{i} Z_{i}^{\prime}$, então, segundo (5) (página 37) as equações resultantes tornam-se:

$$
\begin{aligned}
\operatorname{Tr}\left(V^{-1} Z_{i} Z_{i}^{\prime}\right)=y^{\prime} P V_{i} P y & =\frac{\hat{u}_{i}^{\prime} \hat{u}_{i}}{\sigma_{i}^{4}} \\
& \left.=\operatorname{Tr}\left[R^{-1}-R^{-1} Z\left(Z^{\prime} R^{-1} Z+G^{-1}\right)^{-1} Z^{\prime} R^{-1}\right) Z_{i} Z_{i}^{\prime}\right]
\end{aligned}
$$

e Se $T=\left(Z^{\prime} R^{-1} Z+G^{-1}\right), \quad R=I \sigma_{0}^{2}$ e $\quad G=\sum^{+} I \sigma_{i}^{2}$ em que $\sum^{+}$representa a soma positiva, então,

$$
\operatorname{Tr}\left(V^{-1} V_{i}\right)=\operatorname{Tr}\left(Z_{i}^{\prime} Z_{i}\right) \sigma_{0}^{-2}-\operatorname{Tr}\left(Z_{i}^{\prime} Z_{i} T Z_{i}^{\prime} Z_{i}\right) \sigma_{0}^{-4}
$$

Se $T$ puder ser partida em submatrizes para cada fator aleatório, então

$$
\begin{aligned}
& \left.T{\sigma_{0}^{-2}}^{-2} Z^{\prime} Z+\sum^{+} I \alpha_{i}\right)=I \text { e } T Z^{\prime} Z{\sigma_{0}^{-2}}^{-2}\left(I-T\left(\sum^{+} I \sigma_{i}^{-2}\right) \sigma_{i}^{-2} . \quad\right. \text { Então, } \\
& \operatorname{Tr}\left(Z_{i}^{\prime} Z T Z^{\prime} Z_{i}\right) \sigma_{0}^{-4}=\operatorname{Tr}\left(Z_{i}^{\prime} Z_{i}\right) \sigma_{i}^{-2}-\operatorname{Tr}\left(I-T_{i i}\right) \sigma_{i}^{-2} .
\end{aligned}
$$

Finalmente,

$$
\begin{aligned}
\operatorname{Tr}\left(V^{-1} V_{i}\right) & =\operatorname{Tr}\left(I-T_{i i} \sigma_{i}^{-2}\right){\sigma_{i}^{-2}}^{-2} \operatorname{Tr}\left(\operatorname{I\sigma }_{i}^{-2}\right)-\operatorname{Tr}\left(T_{i i}\right) \sigma_{i}^{-4} \\
& =q_{i} \sigma_{i}^{-2}-\operatorname{Tr}\left(T_{i i}\right) \sigma_{i}^{-4}
\end{aligned}
$$

Considerando (8), tem-se:

$$
\begin{aligned}
\hat{\sigma}_{i}^{2} & =\frac{\hat{u}_{i}^{\prime} \hat{u}_{i}+\operatorname{Tr}\left(T_{i i}\right)}{q_{i}} \operatorname{para} \mathrm{i}=1,2, \ldots, \mathrm{s} \\
\text { e para } \mathrm{i}=0 \quad \hat{\sigma}_{0}^{2} & =\frac{y^{\prime} y-\hat{\beta}^{\prime} X^{\prime} y-\hat{u}^{\prime} Z^{\prime} y}{N}
\end{aligned}
$$

Se houver parentesco entre animais, e representarmos $A$ como a matriz de coeficientes de parentesco entre estes, então, $G=A \otimes G_{i}$, em que $G_{i}$ é a matriz de covariância entre as características $(i=0,1, \ldots, s)$. Neste caso, tem-se de (9):

$$
\hat{\sigma}_{i}^{2}=\frac{\hat{u}_{i}^{\prime} A^{-1} \hat{u}_{i}+\operatorname{Tr}\left(A^{-1} T_{i i}\right)}{q_{i}} \quad \text { para } \mathrm{i}=1,2, \ldots, \mathrm{s} .
$$

Verifica-se em (10) que ML não considera os graus de liberdade decorrentes da estimação dos efeitos fixos, na obtenção de $\sigma_{0}^{2}$, os componentes do erro. 


\subsubsection{Método da Máxima Verossimilhança Restrita - REML}

Embora os estimadores de máxima verossimilhança apresentem ótimas propriedades estatísticas, existem algumas desvantagens na aplicação do método. A primeira delas é que a distribuição dos dados é assumida conhecida. Admite-se, em geral, em análises de dados de áreas biológicas, a distribuição multinormal na estimação dos componentes de covariancia. Uma segunda desvantagem é que os estimadores ML são viciados, uma vez que os efeitos fixos são tratados como se eles fossem conhecidos. A perda dos graus de liberdade resultante da estimação dos efeitos fixos faz com que os estimadores dos componentes de covariancia obtidos pelo método, em geral não coincidam com aqueles estimados pelo método ANOVA ordinário, mesmo quando os dados são balanceados. CORBEIL \& SEARLE (1976) mostram que os estimadores para os componentes do erro podem tornar-se severamente subestimados, especialmente quando o número de classes de efeitos fixos for grande.

$\mathrm{O}$ viés decorrente da perda dos graus de liberdade no ajuste dos efeitos fixos, na estimação dos componentes de covariancia, pode ser removido, considerando-se apenas a parte da função de verossimilhança que independe dos efeitos fixos. Este procedimento pelo qual cada observação é dividida em duas partes independentes, uma referente aos efeitos fixos e outra aos efeitos aleatórios, foi derivada por vários pesquisadores e escolas, mas foi inicialmente sugerido por THOMPSON (1962). Mas foram PATTERSON \& THOMPSON (1971) que desenvolveram formalmente o método, para modelos mistos balanceados ou não. Para dados balanceados, os componentes de covariancia obtidos pelo método são os mesmos encontrados pelos métodos baseados na ANOVA. O procedimento foi denominado método da máxima verossimilhança restrita - REML ("Restricted Maximum Likelihood"). Uma comparação simples entre os métodos ML e REML, é apresentada por HENDERSON (1973) para o modelo $y_{i}=\mu+e_{i}, \mathrm{i}=1, \ldots, \mathrm{n}$. Neste modelo $\operatorname{Var}(e)=I \sigma_{e}^{2}$. O estimador de ML de $\sigma_{e}^{2}$ é $\sum_{i=1}^{n}\left(y_{i}-y_{.}\right)^{2} / N$, e o estimador REML é $\sum_{i=1}^{n}\left(y_{i}-y_{.}\right)^{2} /(N-1)$, com valores esperados $(N-1) \sigma_{e}^{2} / N$ (viciado) e $\sigma_{e}^{2}$ (imparcial), respectivamente.

Para derivação dos estimadores pelo método REML, o procedimento requer que $y$ tenha distribuição multinormal. REML fornece estimadores invariantes à translação, mas em decorrência de se impor a restrição para não negatividade dos componentes de variancia ou dos autovalores das matrizes de covariancia, o procedimento é viciado. Entretanto, o vício é menor do que o existente para o método ML. Para o caso multivariado, o vício pode ser maior se os parâmetros a serem estimados estiverem muito próximos ao limite do espaço de parâmetros. Isto ocorre, por exemplo, quando as matrizes de componentes de covariancias apresentam determinante tendendo a 0 . Neste caso, no processo de estimação dos componentes de covariancia, a convergência será mais lenta (LAIRD \& WARE (1982)).

Todavia, desde que o principal objetivo de se estimar componentes de covariancia na área biológica é para que estes sejam imediatamente utilizados em situações práticas, alguns autores, tais como HENDERSON (1984a), acham que a restrição para não tendenciosidade é menos interessante do que o uso de estimadores no espaço de parâmetros. 
A principal diferença entre os métodos ML e REML é que o primeiro usa a função de verossimilhança de $y$ ou o logarıtmo desta função, enquanto o segundo adota a função de verossimilhança de $L^{\prime} y$, um conjunto de contrastes de erros (com esperança nula) que representa efetivamente as observações ajustadas para os efeitos fixos.

A desvantagem do método REML, que é também uma desvantagem adicional do método ML, é que o procedimento de estimação em geral requer esquemas iterativos e a convergência além de ser lenta não está garantida. Existem, entretanto alguns algoritmos e outros procedimentos que procuram melhorar a velocidade da convergencia. $\mathrm{O}$ método $\Delta^{2}$ de Aitken é um destes métodos e foi apresentado na seção 2.2 (pág. 26). Sob normalidade as estimativas I-MINQUE $\cong \mathrm{REML}$. Além disso, na primeira iteração REML $\cong$ MINQUE $\cong$ MIVQUE (SEARLE, 1979).

Existem quatro formas de derivação das equações pelo método REML. A primeira forma consiste em tomar as formas quadráticas utilizadas na estimação pelo método MIVQUE e derivar novos valores esperados, assumindo que os valores preliminares são iguais aos verdadeiros valores dos parâmetros. Assim, sob normalidade, na primeira iteração para obtenção dos componentes pelo método REML, estes estimadores são os mesmos obtidos pelo método MINQUE OU MIVQUE (SEARLE, 1979).

A segunda forma de obtenção dos estimadores dos componentes de covariância utilizando REML é partindo-se das equações derivadas pelo método ML. A terceira forma parte-se da função de verossimilhança restrita e diferencia-se para obtenção do máximo com respeito aos parâmetros desconhecidos. Finalmente, na quarta forma adota-se o método livre de derivadas. Nesse processo tenta-se encontrar o máximo da função de verossimilhança restrita através de procedimentos de procura sequencial ou linear.

\subsubsection{Derivação dos componentes de variancia pelo método REML}

\subsection{REML a partir das equações MIVQUE}

De (6), (pág. 39), tem-se:

$$
\begin{aligned}
E\left(\hat{u}_{i}^{\prime} \hat{u}_{i}\right)=\left(q_{i}-2 \operatorname{Tr}\left(c^{i i} \alpha_{i}\right)\right. & \left.+\operatorname{Tr}\left(\left(c^{i i}\right)^{2} \alpha_{i}^{2}\right)\right) \sigma_{i}^{2}+\sum_{j \neq i}^{s} \operatorname{Tr}\left(c^{i j} c^{i j} \alpha_{j}^{2}\right) \sigma_{j}^{2} \\
& +\left(\operatorname{Tr}\left(c^{i i}\right)-\operatorname{Tr}\left(\left(c^{i i}\right)^{2} \alpha_{i}\right)-\sum_{j=1}^{s} \operatorname{Tr}\left(c^{i j} c^{i j} \alpha_{j}^{2}\right)\right) \sigma_{0}^{2}
\end{aligned}
$$

em que $\alpha_{i}=G_{i}^{-1} G_{0}$ sendo $G_{0}$ e $G_{i}$ valores iniciais para $\sigma_{0}^{2}$ e $\sigma_{i}^{2}$, respectivamente. Para derivar os estimadores REML, assume-se que $\alpha_{i}=\sigma_{0}^{2} / \sigma_{i}^{2}$ e substitui-se $\alpha_{i}$ na expressão acima. Fazendo a substituição e a devida simplificação, resulta:

$$
\begin{aligned}
E\left(\hat{u}_{i}^{\prime} \hat{u}_{i}\right) & =q_{i} \sigma_{i}^{2}-\operatorname{Tr}\left(c^{i i}\right) \sigma_{0}^{2} . \text { Então } \\
\hat{\sigma}_{i}^{2} & =\left(\frac{\hat{u}_{i}^{\prime} \hat{u}_{i}+\operatorname{Tr}\left(c^{i i}\right) \hat{\sigma}_{0}^{2}}{q_{i}}\right) .
\end{aligned}
$$


Havendo relação de parantesco entre individuos, $G=A \otimes G_{i}$, para $A$ a matriz de parentesco entre os animais e $G_{i}$ a matriz de covariancias entre as caracteristicas. Neste caso,

$$
\hat{\sigma}_{i}^{2}=\left(\frac{\hat{u}_{i}^{\prime} A^{-1} \hat{u}_{i}+\operatorname{Tr}\left(A^{-1} c^{i i}\right) \hat{\sigma}_{0}^{2}}{q_{i}}\right) .
$$

Observe que as quantidades envolvidas são sempre positivas, pois $\hat{u}_{i}^{\prime} \hat{u}_{i}$ ou $\hat{u}_{i}^{\prime} A^{-1} \hat{u}_{i}$ m é uma soma de quadrados e $\operatorname{Tr}\left(c^{i i}\right)$ ou $\operatorname{Tr}\left(A^{-1} c^{i i}\right)$ envolve elementos diagonais de $C$ ou de $A^{-1} C$, e representa as variâncias dos erros de predição $\left(\operatorname{Var}\left(\hat{u}_{i}-u_{i}\right)\right)$, sendo ambas positivas. Assim, $\hat{\sigma}_{i}^{2}$ está sempre no espaço de parâmetro. Da mesma forma, os valores esperados da equação MIVQUE, $y^{\prime} y-\hat{\beta}^{\prime} X^{\prime} y-\sum_{i=1}^{s} \hat{u}_{i}^{\prime} Z_{i}^{\prime} y$, se reduzem a

$$
E\left(y^{\prime} y-\hat{\beta}^{\prime} X^{\prime} y-\sum_{i=1}^{s} \hat{u}_{i}^{\prime} Z_{i}^{\prime} y\right)=(N-r(X)) \sigma_{0}^{2}
$$

e, sob a suposição de que $\alpha_{i}=\sigma_{0}^{2} / \sigma_{i}^{2}$, o estimador REML para $\hat{\sigma}_{0}^{2}$ é:

$$
\hat{\sigma}_{0}^{2}=\frac{y^{\prime} y-\hat{\beta}^{\prime} X^{\prime} y-\sum_{i=1}^{s} \hat{u}_{i}^{\prime} Z_{i}^{\prime} y}{N-r(X)} .
$$

SCHAEFFER (1993) cita que as expressões dos valores esperados assim obtidas podem ser imaginados como falsas esperanças. As fórmulas para $\hat{\sigma}_{i}^{2}$ e $\hat{\sigma}_{0}^{2}$ são conhecidas como algorftimo EM, ou seja, maximização de esperanças, que é um algorftmo amplamente utilizado, o qual foi descrito por DEMPSTER et al. (1977).

As estimativas REML são obtidas usando-se $\hat{\sigma}_{i}^{2}$ e $\hat{\sigma}_{0}^{2}$ para formar novas razões, $\hat{\alpha}_{i}$, e estas serão utilizadas como estimativas preliminares para calcular um novo $\hat{\alpha}_{i}$, até a razão usada como valor preliminar coincidir com a razão estimada. Com o uso do algorftmo EM, para obter as estimativas pelo método REML, a convergência não está garantida. Isto pode ocorrer especialmente quando os componentes de variancia tendem a ser negativos ou quando as matrizes de covariancia tendem a tornar-se não positivas definidas. Mesmo se o procedimento convergir, as estimativas obtidas podem não representar aquelas que maximizam a função de verossimilhança restrita, em todo espaço paramétrico. GROENEVELD \& KOVAK (1990) encontraram três diferentes soluções para um conjunto de dados na presença de máximos locais. No entanto, a convergência normalmente ocorrerá se o número de observações for grande e esta corresponderá provavelmente ao máximo da função de verossimilhança. Modelos com poucas observações e muitos fatores aleatórios podem apresentar problemas de convergência.

\subsection{REML a partir das equações de verossimilhança}

De (8), (pág. 42) tem-se

$$
\operatorname{Tr}\left(V^{-1} Z_{i} Z_{i}^{\prime}\right)=y^{\prime} P Z_{i} Z_{i}^{\prime} P y \quad \text { para } \mathrm{i}=1,2, \ldots, \mathrm{s} .
$$

Substituindo-se $y, X, Z$ e $V$, respectivamente, por $L^{\prime} y, \emptyset, L^{\prime} Z$ e $L^{\prime} V L$ nas equaç̃es em (14) e sabendo-se que $L^{\prime} X=\emptyset$, tem-se:

$$
\operatorname{Tr}\left(L^{\prime} V L\right)^{-1} L^{\prime} Z_{i} Z_{i}^{\prime} L=y^{\prime} L\left(L^{\prime} V L\right)^{-1} L^{\prime} Z_{i} Z_{i}^{\prime} L\left(L^{\prime} V L\right)^{-1} L^{\prime} y
$$


e como $L\left(L^{\prime} V L\right)^{-1} L^{\prime}=P$ (SEARLE, 1979, pág. 94), então

$$
\begin{aligned}
\operatorname{Tr}\left(P Z_{i} Z_{i}^{\prime}\right) & =y^{\prime} P Z_{i} Z_{i}^{\prime} P y \text { para i=0,1,..,s. } \\
& =(y-X \hat{\beta}) V^{-1} Z_{i} Z_{i}^{\prime} V^{-1}(y-X \hat{\beta}) . \text { Em que } \\
(y-X \hat{\beta}) & =\left(I-X\left(X^{\prime} V^{-1} X\right)^{-} X^{\prime} V^{-1}\right) y=V P y \\
\Rightarrow \operatorname{Tr}\left(P Z_{i} Z_{i}^{\prime}\right) & =\operatorname{Tr}\left(P V P Z_{i} Z_{i}^{\prime}\right)=\operatorname{Tr}\left(P Z_{i} Z_{i}^{\prime} P V\right) \\
& =\operatorname{Tr}\left(P Z_{i} Z_{i}^{\prime} P \sum_{j=0}^{s} \sigma_{j}^{2} Z_{j} Z_{j}^{\prime}\right) \\
& =\sum_{j=0}^{s} \operatorname{Tr}\left(P Z_{i} Z_{i}^{\prime} P Z_{j} Z_{j}^{\prime}\right) \sigma_{j}^{2} .
\end{aligned}
$$

Então, a equação (14) pode ser reescrita como:

$$
\operatorname{Tr}\left(P Z_{i} Z_{i}^{\prime} P Z_{j} Z_{j}^{\prime}\right) \sigma_{i}^{2}=y^{\prime} P Z_{i} Z_{i}^{\prime} P y \text { para } \mathrm{i}, \mathrm{j}=0, \ldots, \mathrm{s} .
$$

SEARLE (1979, pág. 92) mostra uma forma interessante para obtenção de $\hat{\sigma}_{0}^{2}$. Para isto, seja $\mathrm{i}=0$ em $(15)$, com $Z_{0} Z_{0}^{\prime}=I$, então

$$
\operatorname{Tr}(P)=y^{\prime} P P y \text {. }
$$

Multiplicando-se (15) por $\sum_{i=1}^{s} \hat{\sigma}_{i}^{2}$, tem-se, $\operatorname{Tr}\left(P \sum_{i=1}^{s} \hat{\sigma}_{i}^{2} Z_{i} Z_{i}^{\prime}\right)=y^{\prime} P \sum_{i=1}^{s} \hat{\sigma}_{i}^{2} Z_{i} Z_{i}^{\prime} P y$. E, sabendo-se que $\sum_{i=1}^{s} \hat{\sigma}_{i}^{2} Z_{i} Z_{i}^{\prime}=V-\hat{\sigma}_{0}^{2} I$, então

$$
\operatorname{Tr}(P V)-\hat{\sigma}_{0}^{2} \operatorname{Tr}(P)=y^{\prime} P V P y-\hat{\sigma}_{0}^{2} y^{\prime} P^{2} y .
$$

Além disso, como $\operatorname{Tr}(P V)=r(P V)=r(P)=N-r(X), P V P=P$ e utilizando-se (17), tem-se, a partir de (18):

$$
\begin{aligned}
N-r(X) & =(y-X \hat{\beta})^{\prime} V^{-1}(y-X \hat{\beta}), \text { resultando finalmente em } \\
\hat{\sigma}_{0}^{2} & =\frac{(y-X \hat{\beta}) H^{-1}(y-X \hat{\beta})}{(N-r(X))}
\end{aligned}
$$

para $V=\sigma_{0}^{2} H$ e $H=\sum_{i=1}^{s} Z_{i} Z_{i}^{\prime} \psi+I_{N}$ em que $\psi=\sigma_{i}^{2} / \sigma_{0}^{2}=1 / \alpha_{i}$ (SEARLE, 1979, pág. 30)

\subsection{REML a partir da função de verossimilhança}

Por ser este item de maior importância para os desenvolvimentos posteriores, faremos uma breve revisão de algumas propriedades de determinantes de matrizes, as quais serão utilizadas nas deduções subseqüentes. Detalhes adicionais podem ser encontrados, por exemplo, em SEARLE (1979), SEARLE (1982) e SCHAEFFER (1993).

Seja o modelo linear misto, já considerado em (4), (pág. 27), com as mesmas pressuposições:

$$
\begin{aligned}
y & =X \beta+Z u+\varepsilon \text { e seja } \\
P & =V^{-1}-V^{-1} X\left(X^{\prime} V^{-1} X\right)^{-} X^{\prime} V^{-1} \text { a matriz projeção. }
\end{aligned}
$$


Para $y$ univariado

$$
\begin{aligned}
V & =\sum_{i=1}^{s} Z_{i} Z_{i}^{\prime} \sigma_{i}^{2}+I \sigma_{0}^{2} \\
\frac{\partial V^{-1}}{\partial \sigma_{i}^{2}} & =-V^{-1} \frac{\partial V}{\partial \sigma_{i}^{2}} \\
\frac{\partial \log |V|}{\partial \sigma_{i}^{2}} & =\operatorname{Tr}\left(V^{-1} \frac{\partial V}{\partial \sigma_{i}^{2}}\right) \\
\frac{\partial P}{\partial \sigma_{i}^{2}} & =-P \frac{\partial V}{\partial \sigma_{i}^{2}} P \\
\frac{\partial V}{\partial \sigma_{i}^{2}} & =Z_{i} Z_{i}^{\prime}, \\
\frac{\partial \log \left|X^{\prime} V^{-1} X\right|}{\partial \sigma_{i}^{2}} & =\operatorname{Tr}\left(X^{\prime} V^{-1} X\right)^{-} X^{\prime} V^{-1} \frac{\partial V}{\partial \sigma_{i}^{2}} V^{-1} X, \mathrm{e} \\
\frac{\partial \log |V|}{\partial \sigma_{i} \partial \sigma_{j}} & =\operatorname{Tr}\left(V^{-1} \frac{\partial V}{\partial \sigma_{i}} \frac{\partial V}{\partial \sigma_{j}}\right) .
\end{aligned}
$$

Sejam $A, B, D$ e $Q$ matrizes quaisquer, com $A$ e $D$ não singular. Então:

$$
\left|\begin{array}{ll}
A & B \\
Q & D
\end{array}\right|=|A|\left|D-Q A^{-1} B\right|=|D|\left|A-B D^{-1} Q\right| \text {. }
$$

Se $\theta$ é um escalar, então $|\theta A|=\theta^{m}|A|$ em que m é a ordem da matriz $A$. Seja

$$
C=\left(\begin{array}{ll}
X^{\prime} R^{-1} X & X^{\prime} R^{-1} Z \\
Z^{\prime} R^{-1} X & Z^{\prime} R^{-1} Z+G^{-1}
\end{array}\right)
$$

para $X$ uma matriz de posto coluna completo. Então, de acordo com (19),

$$
\begin{aligned}
|C| & =\mid X^{\prime} R^{-1} X \| G^{-1}+Z^{\prime}\left(R^{-1}-R^{-1} X\left(X^{\prime} R^{-1} X\right)^{-1} X^{\prime} R^{-1} Z \mid\right. \\
& =\left|Z^{\prime} R^{-1} Z+G^{-1} \| X^{\prime}\left(R^{-1}-R^{-1} Z\left(Z^{\prime} R^{-1} Z\right)+G^{-1}\right)^{-1} Z^{\prime} R^{-1} X\right| \\
\text { e se } S & =R^{-1}-R^{-1} X\left(X^{\prime} R^{-1} X\right)^{-1} X^{\prime} R^{-1}, \text { então } \\
|C| & =\left|X^{\prime} R^{-1} X\left\|G^{-1}+Z^{\prime} S Z|=| Z^{\prime} R^{-1} Z+G^{-1}\right\| X^{\prime} V^{-1} X\right|
\end{aligned}
$$

Observa-se que

$$
\begin{gathered}
|V|=\left|R+Z G Z^{\prime}\right|=\left|R\left(I+R^{-1} Z G Z^{\prime}\right)\right|=|R|\left|I+R^{-1} Z G Z^{\prime}\right| \\
=\left|R\left\|I+Z^{\prime} R^{-1} Z G|=| R\right\|\left(G^{-1}+Z^{\prime} R^{-1} Z\right) G\right| \\
=\left|R\left\|G^{-1}+Z^{\prime} R^{-1} Z\right\| G\right|
\end{gathered}
$$


Se $L$ representa contrastes de erro com esperança nula, isto é, $L^{\prime} X=\emptyset \Rightarrow \mathrm{E}\left(L^{\prime} y\right)=$ $\mathrm{E}\left(L^{\prime} X \beta\right)=\emptyset$. Seja por exemplo $L=I-X\left(X^{\prime} V^{-1} X\right)^{-} X^{\prime}$, então,

$$
\begin{gathered}
\left|L^{\prime} V L\right|=|V|\left|X^{\prime} V^{-1} X\right| \mathrm{e} \\
\log \left(\left|L^{\prime} V L\right|\right)=\log |V|+\log \left|X^{\prime} V^{-1} X\right| \\
y^{\prime} L\left(L^{\prime} V L\right)^{-1} L^{\prime} y=y^{\prime}\left(V^{-1}-V^{-1} X\left(X^{\prime} V^{-1} X\right)^{-} X^{\prime} V^{-1}\right) y=y^{\prime} P y \\
=(y-X \hat{\beta})^{\prime} V^{-1}(y-X \hat{\beta})
\end{gathered}
$$

Os estimadores dos componentes de covariancia obtidos pelo método REML são derivados a partir de uma função de verossimilhança para um conjunto de contrastes de erros $\left(L^{\prime} y\right)$ das observações, com $L^{\prime} X=\emptyset$. A função de verossimilhança restrita, para o modelo em (4), (pág. 27), é dada por:

$$
\Lambda_{1}=(2 \pi)^{-\frac{1}{2}(N-r(X))}\left|L^{\prime} V L\right|^{-\frac{1}{2}} e^{-\frac{1}{2}\left(L^{\prime} y\right)^{\prime}\left(L^{\prime} V L\right)^{-1}\left(L^{\prime} y\right)} .
$$

Tomando-se o logaritmo natural desta função, obtém-se:

$$
\begin{aligned}
L_{1} & =-\frac{1}{2}\left((N-r(X)) \log (2 \pi)+\log \left|L^{\prime} V L\right|+\left(L^{\prime} y\right)^{\prime}\left(L^{\prime} V L\right)^{-1}\left(L^{\prime} y\right)\right) \\
& =-\frac{1}{2}\left((N-r(X)) \log (2 \pi)+\log \left|L^{\prime} V L\right|+y^{\prime} L\left(L^{\prime} V L\right)^{-1} y^{\prime} L\right) .
\end{aligned}
$$

Note-se que $-0.5(N-r(X)) \log (2 \pi)$ é uma constante que independe dos componentes de covariancia desconhecidos ou fatores do modelo e portanto pode ser ignorada. Considerando-se (22) e (23), $L_{1}$, pode ser reescrito como:

$$
\begin{aligned}
L_{2}= & -\frac{1}{2}\left((N-r(X)) \log (2 \pi)+\log |V|+\log \left|X^{\prime} V^{-1} X\right|\right) \\
& -\frac{1}{2}\left((y-X \hat{\beta})^{\prime} V^{-1}(y-X \hat{\beta})\right)
\end{aligned}
$$

mas de (21) $\log |V|=\log |R|+\log |G|+\log \left|G^{-1}+Z^{\prime} R^{-1} Z\right|$. E a partir de (20) $\log \left|X^{\prime} V^{-1} X\right|=\log |C|-\log \left|Z^{\prime} R^{-1} Z+G^{-1}\right|$. Substituindo-se estes resultados em (24), obtêm-se:

$$
L_{2}=-\frac{1}{2}\left((N-r(X)) \log (2 \pi)+\log |R|+\log |G|+\log |C|+y^{\prime} P y\right) .^{4}
$$

Este resultado é fundamental para obtenção dos estimadores dos componentes de covariancia adotando-se o método livre de derivadas.

Em análise univariada, a situação é bem simplificada. Neste caso,

$$
\begin{aligned}
& \log |R|=\log \left|I \sigma_{0}^{2}\right|=N \log \left|\sigma_{0}^{2}\right| \\
& \log |G|=\sum_{i=1}^{s} q_{i} \log \sigma_{i}^{2} \\
& \log |C|=\log \left|X^{\prime} R^{-1} X\right|+\log \left|Z^{\prime} S Z+G^{-1}\right|
\end{aligned}
$$

\footnotetext{
${ }^{4}$ Denotaremos por $C$ a matriz de coeficientes $(C)$, após imposição das restriçóes não estimáveis em $X$
} 


$$
\text { em que } \begin{aligned}
\log \left|X^{\prime} R^{-1} X\right|=\log \left|X^{\prime} X \sigma_{0}^{-2}\right| & =\log \left(\sigma_{0}^{-2}\right)^{r(X)}\left|X^{\prime} X\right| \\
& =\log \left|X^{\prime} X\right|-r(X) \log \left(\sigma_{0}^{2}\right) \\
Z^{\prime} S Z+G^{-1}=\sigma_{o}^{-2} Z^{\prime} M Z+G^{-1} & =\sigma_{0}^{-2}\left(Z^{\prime} M Z+G^{-1} \sigma_{0}^{2}\right) . \text { Assim, } \\
\log |C| & =\log \left|X^{\prime} X\right|-r(X) \log \sigma_{0}^{2}-q \log \sigma_{0}^{2} \\
& \left.+\log \left|Z^{\prime} M Z+G^{-1} \sigma_{0}^{2}\right|\right)
\end{aligned}
$$

e, finalmente,

$$
\begin{aligned}
L_{2}= & -\frac{1}{2}\left((N-r(X)) \log (2 \pi)+(N-r(X)-q) \log \sigma_{0}^{2}\right. \\
& \left.+\sum_{i=1}^{s} q_{i} \log \sigma_{i}^{2}+\log |C|+y^{\prime} P y\right)
\end{aligned}
$$

em que

$$
C=\left(\begin{array}{ll}
X^{\prime *} X^{*} & X^{\prime *} Z \\
Z^{\prime} X^{*} & Z^{\prime} Z+G^{-1} \sigma_{0}^{2}
\end{array}\right)
$$

com $X^{*}$ de posto coluna completo

Os estimadores dos componentes de variancia, neste caso, podem ser obtidos por derivação ou pelo método livre de derivadas.

\section{a) Por Derivação}

$$
\begin{aligned}
\frac{\partial L_{2}}{\partial \sigma_{i}^{2}}= & -\frac{1}{2}\left(\operatorname{Tr}\left(V^{-1} \frac{\partial V}{\partial \sigma_{i}^{2}}\right)-\operatorname{Tr}\left(X^{\prime} V^{-1} X\right)^{-} X Z^{\prime} V^{-1} \frac{\partial V}{\partial \sigma_{i}^{2}} V^{-1} X\right) \\
& +\frac{1}{2}\left((y-X \hat{\beta})^{\prime} V^{-1} \frac{\partial V}{\partial \sigma_{i}^{2}} V^{-1}(y-X \hat{\beta})\right)
\end{aligned}
$$

Combinando os termos envolvendo traços e observando que $V^{-1}(y-X \hat{\beta})=P y$, tem-se:

$$
\begin{aligned}
\frac{\partial L_{2}}{\partial \sigma_{i}^{2}} & =-\frac{1}{2}\left(\operatorname{Tr}\left(V^{-1}-V^{-1} X\left(X^{\prime} V^{-1} X\right)^{-} X^{\prime} V^{-1} \frac{\partial V}{\partial \sigma_{i}^{2}}\right)-y^{\prime} P \frac{\partial V}{\partial \sigma_{i}^{2}} P y\right) \\
& =-\frac{1}{2}\left(\operatorname{Tr}\left(P Z_{i} Z_{i}^{\prime}\right)-y^{\prime} P Z_{i} Z_{i}^{\prime} P y\right) \text { para } \mathrm{i}=1,2, \ldots, \mathrm{s}, \text { ou } \\
& =-\frac{1}{2}\left(\operatorname{Tr}(P)-y^{\prime} P P y\right) \quad \text { para } \mathrm{i}=0, \text { para o componente residual. }
\end{aligned}
$$

Observe que

$$
\operatorname{Tr}\left(P Z_{i} Z_{i}^{\prime}\right)=q_{i} \sigma_{i}^{2}-\operatorname{Tr}\left(c^{i i} \sigma_{0}^{2}\right) / \sigma_{i}^{-4}
$$

e

$$
\operatorname{Tr}(P)=(N-r(X)) \sigma_{0}^{2}-\sum_{i=1}^{s} \hat{u}_{i}^{\prime} \hat{u}_{i} \alpha_{i}
$$


Igualando as derivadas a zero, tem-se:

$$
q_{i} \hat{\sigma}_{i}^{2}-\frac{\operatorname{Tr}\left(c^{i i}\right) \hat{\sigma}_{0}^{2}}{\hat{\sigma}_{i}^{-4}}=\frac{\hat{u}_{i}^{\prime} \hat{u}_{i}}{\hat{\sigma}_{i}^{-4}}
$$

que resulta em

$$
\Rightarrow \hat{\sigma}_{i}^{2}=\frac{\hat{u}_{i}^{\prime} \hat{u}_{i}+\operatorname{Tr}\left(c^{i i}\right) \hat{\sigma}_{0}^{2}}{q_{i}}
$$

e

$$
\begin{aligned}
& (N-r(X)) \hat{\sigma}_{0}^{2}-\sum_{i=1}^{s} \hat{u}_{i}^{\prime} \hat{u}_{i} \alpha_{i}=y^{\prime} P y-\sum_{i=1}^{s} \hat{u}_{i}^{\prime} \hat{u}_{i} \alpha_{i} \\
& \Rightarrow \hat{\sigma}_{0}^{2}=\frac{y^{\prime} P y}{(N-r(X))}=\frac{y^{\prime} y-\hat{\beta}^{\prime} X^{\prime} y-\hat{u}^{\prime} Z^{\prime} y}{(N-r(X))}
\end{aligned}
$$

Da mesma forma que foi citado anteriormente, se houver parentesco entre animais e considerando-se que $A$ é a matriz de relacionamentos entre animais, então de (28)

$$
\Rightarrow \hat{\sigma}_{i}^{2}=\frac{\hat{u}_{i}^{\prime} A^{-1} \hat{u}_{i}+\operatorname{Tr}\left(A^{-1} c^{i i}\right) \hat{\sigma}_{0}^{2}}{q_{i}} .
$$

Os resultados de (27) e de (28) são os mesmos já obtidos em (13) e (12).

\section{b) Método Livre de Derivadas (DF)}

O método livre de derivadas foi proposto por SMITH \& GRASER (1986) e GRASER et al. (1987), para estimação de componentes de variância, em análise univariada, e foi expandido para o caso multivariado por MEYER (1989, 1991 e 1993), que desenvolveu um conjunto de programas para o cálculo de componentes de covariância para uma ampla variedade de condições.

Em resumo, imagine uma superfície s dimensional, contendo os valores da função de verossimilhança para todos possfveis conjuntos de valores das razões dos componentes de variância. em relação à variancia residual. A técnica consiste em procurar a superfície e encontrar o conjunto de razões de variâncias para o qual a função de verossimilhança é maximizada. Existem várias maneiras de se conduzir a procura. Cuidado tem que ser tomado para encontrar o máximo global da função. Também deve-se utilizar um procedimento tal que o número de avaliações da verossimilhança na procura do máximo seja minimizado. $\mathrm{O}$ procedimento proposto sugere a formação da matriz:

$$
M=\left[\begin{array}{cccc}
X^{\prime} X & X^{\prime} Z & \vdots & X^{\prime} y \\
Z^{\prime} X & Z^{\prime} Z+G^{-1} \sigma_{0}^{2} & \vdots & Z^{\prime} y \\
\cdots & \cdots & \cdots & \cdots \\
y^{\prime} X & y^{\prime} Z & \vdots & y^{\prime} y
\end{array}\right]=\left[\begin{array}{ccc}
C & \vdots & W^{\prime} y \\
\cdots & \cdots & \cdots \\
y^{\prime} W & \vdots & y^{\prime} y
\end{array}\right]
$$


e, por eliminação de Gauss (veja (2.1.1.2), pág. 8), uma linha por vez, e a soma do logarítmo dos pivôs não nulos (utilizando a mesma ordem para cada avaliação da verossimilhança), será obtido $\log |C|$ e $y^{\prime}(y-X \hat{\beta}-Z \hat{u})=y^{\prime} P y$ de (26). A eliminação de Gauss, utilizando a técnica de matrızes esparsas, requer menor tempo de processamento do que a inversão da matriz de coeficientes das equações do modelo misto (SCHAEFFER, 1993), além de permitir que se trabalhe com matrizes de altas dimensões. A ordem dos fatores dentro das equações poderá ser crítico para o processo de cálculo (BOLDMAN et al. (1993)). A função de verossimilhança pode ser avaliada sem o cálculo de soluções para as equações do modelo linear misto, sem a necessidade de inversão da matriz de coeficientes do modelo e sem computar qualquer dos $\sigma_{i}^{2}$. Neste procedimento, em cada avaliação obtém-se $y^{\prime} P y$ e $\log |C|$ por eliminação de Gauss e, a partir dal, basta encontrar de (26), $\sigma_{i}^{2}$ e $\sigma_{0}^{2}$ ou de (25) $|R|$ e $|G|$ que maximizam $L_{2}$, respectivamente, para o caso uni ou multivariado.

Procedimento similar para obtenção de $\log |C|$ e $y^{\prime} P y$ foi sugerido por BOLDMAN \& VAN VLECK (1991), que recomendam a utilização da decomposição de Cholesky da matriz de coeficientes $(C)$ para estimação de componentes de covariancia em análise multivariada, adotando-se o método REML. No caso, a matriz de coeficientes $C$ é decomposta no produto de matrizes triangulares inferiores $L=\left[l_{i j}\right] \operatorname{com} l_{i j}=0$ para $j>i$ e suas transpostas. Para que o método seja aplicado, deve-se impor restrições paramétricas na formação da matriz $C$, tornando-a positiva definida, o que implica em se tomar uma submatriz de $C$ de posto completo. Então, para

$$
C=\left(\begin{array}{ll}
X^{\prime} R^{-1} X & X^{\prime} R^{-1} Z \\
Z^{\prime} R^{-1} X & Z^{\prime} R^{-1} Z+G^{-1}
\end{array}\right)
$$

tem-se, após imposição das restriçẽs na matriz $X$,

$$
L L^{\prime}=\left(\begin{array}{ll}
X^{\prime *} R^{-1} X^{*} & X^{\prime *} R^{-1} Z \\
Z^{\prime} R^{-1} X^{*} & Z^{\prime} R^{-1} Z+G^{-1}
\end{array}\right)
$$

e como o determinante de uma matriz triangular é simplesmente o produto de seus elementos diagonais, resulta que $\log |C|=2 \sum_{i=1}^{r(C)} \log \left(l_{i i}\right)$, para $l_{i i} \neq 0$. Em seguida por meio de substituições "para frente" e retrosubstituições, obtêm-se as soluções para os efeitos fixos e aleatórios do modelo linear misto, e $y^{\prime} P y$ é calculado como: $y^{\prime} P y=y^{\prime} R^{-1} y$ $y^{\prime} R^{-1} X^{*} \hat{\beta}^{*}-y^{\prime} R^{-1} Z \hat{u}$.

Segundo MEYER (1993) e BOLDMAN et al. (1993), a decomposição de Cholesky não é mais rápida do que a eliminação de Gauss (GE), mas a grande vantagem é que ela encontra-se implementada nas principais rotinas de operação com matrizes esparsas, tais como a SPARSPAK (GEORDE et al., 1980 e GEORDE et al., 1981) e a FSPAK (PEREZ-ENCIZO \& MISZTAL, 1992)).

$\mathrm{Na}$ seção material e métodos serão apresentadas formas explícitas para estimação dos componentes de covariancia por este método. 


\subsubsection{Método da Falsa Esperança}

Falsas esperanças são tomadas, assumindo-se que os valores preliminares das razões de variância são iguais aos verdadeiros valores. Esta aproximação é a mesma utilizada para derivar fórmulas pelo método EM-REML, a partir das equações derivadas do método MIVQUE. SCHAEFFER (1986) encontrou um grupo de formas quadráticas, cujas falsas esperanças não envolvem os elementos inversos das equações do modelo misto, com estimativas sempre positivas. Os estimadores resultantes são invariantes à translação e viciados. A maior vantagem desses estimadores é que suas estimativas podem ser obtidas com pouca dificuldade computacional. VanRADEN (1986) expandiu as formas quadráticas obtidas por SCHAEFFER (1986), para o caso de parentesco entre animais, as quais poderiam também ser utilizadas em modelos lineares básicos, para estimação de componentes de covariancia. Por outro lado, Ouweltjes et al. (1988), citados por SCHAEFFER (1993) verificaram que o método da falsa esperança não foi capaz de considerar a seleção tipo refugo (ao contrário do método REML), produzindo nestes casos estimativas viciadas para os componentes de covariancia.

\section{- Derivação do Método}

Considerando apenas o caso univariado, o primeiro passo é a absorção dos efeitos fixos nas equações para os efeitos aleatórios do modelo misto. 0 lado direito das equações absorvidas para o $i^{\text {ésimo }}$ fator é dado por: $Z_{i}^{\prime} M y$ em que $M=I-X\left(X^{\prime} X\right)^{-} X^{\prime}$. 0 valor esperado de $Z_{i}^{\prime} M y$ é nulo e a matriz de variância e covariância de $Z^{\prime} M y$ é $V\left(Z^{\prime} M y\right)$ $=\sum_{j=1}^{s}\left(Z^{\prime} M Z_{j}\right)\left(Z_{j}^{\prime} M Z\right) \sigma_{j}^{2}+Z^{\prime} M Z \sigma_{0}^{2}$. O vetor solução para os fatores aleatórios é:

$$
\left(\begin{array}{l}
\hat{u}_{1} \\
\hat{u}_{2} \\
\vdots \\
\hat{u}_{s}
\end{array}\right)=\left(\begin{array}{cccc}
Z_{1}^{\prime} M Z_{1}+I \alpha_{1} & Z_{1}^{\prime} M Z_{2} & \cdots & Z_{1}^{\prime} M Z_{s} \\
Z_{2}^{\prime} M Z_{1} & Z_{2}^{\prime} M Z_{2}+I \alpha_{2} & \cdots & Z_{1}^{\prime} M Z_{s} \\
\vdots & \vdots & \vdots & \\
Z_{s}^{\prime} M Z_{1} & Z_{s}^{\prime} M Z_{2} & \cdots & Z_{s}^{\prime} M Z_{s}+I \alpha_{s}
\end{array}\right)^{-1}\left(\begin{array}{c}
Z_{1}^{\prime} M y \\
Z_{2}^{\prime} M y \\
\vdots \\
Z_{s}^{\prime} M y
\end{array}\right)
$$

As formas quadráticas propostas são:

$$
\hat{u}_{i}^{\prime} Z_{i}^{\prime} M y
$$

e

$$
y^{\prime} y-\hat{\beta}^{\prime} X^{\prime} y-\sum_{i=1}^{s} \hat{u}_{i}^{\prime} Z_{i}^{\prime} y=y^{\prime} M y-\sum_{i=1}^{s} \hat{u}_{i}^{\prime} Z_{i}^{\prime} M y .
$$

Esta última forma quadrática corresponde à mesma obtida pelo método REML, e tem falsa esperança igual a

$$
E\left(y^{\prime} y-\hat{\beta}^{\prime} X^{\prime} y-\hat{u}_{i}^{\prime} Z_{i}^{\prime} y\right)=(N-r(X)) \sigma_{0}^{2}
$$

que resultará em:

$$
\hat{\sigma}_{0}^{2}=\frac{y^{\prime} y-\hat{\beta}^{\prime} X^{\prime} y-\hat{u}^{\prime} Z^{\prime} y}{N-r(X)} \quad \text { ou } \quad \hat{\sigma}_{0}^{2}=\frac{y^{\prime} M y-\hat{u}^{\prime} Z^{\prime} M y}{N-r(X)}
$$


O valor esperado usual para $\hat{u}_{i}^{\prime} Z_{i}^{\prime} M y$ é:

$$
\begin{aligned}
E\left(\hat{u}_{i}^{\prime} Z_{i}^{\prime} M y\right)=\operatorname{Tr}\left(Z_{i}^{\prime} M Z_{i}\right)-\operatorname{Tr}\left(c^{i i} Z_{i}^{\prime} M Z_{i} \alpha_{i}\right) \sigma_{i}^{2} & -\sum_{j \neq i}^{s} \operatorname{Tr}\left(c^{i j} Z_{j}^{\prime} M Z_{i} \alpha_{j}\right) \sigma_{j}^{2} \\
& +\left(q_{i}-\operatorname{Tr}\left(c^{i i}\right) \alpha_{i}\right) \sigma_{0}^{2}
\end{aligned}
$$

Quando os $\alpha_{i}^{\prime} s$ são tomados como razões de verdadeiros valores, $E\left(\hat{u}_{i}^{\prime} Z_{i}^{\prime} M y\right)=\operatorname{Tr}\left(Z_{i}^{\prime} M Z_{i}\right) \sigma_{i}^{2}$. Neste caso,

$$
\hat{\sigma}_{i}^{2}=\frac{\hat{u}^{\prime} Z_{i}^{\prime} M y}{\operatorname{Tr}\left(Z_{i}^{\prime} M Z_{i}\right)} .
$$

Observe que o estimador de $\sigma_{i}^{2}$ é sempre positivo. Além disso, $\operatorname{Tr}\left(Z_{i}^{\prime} M Z_{i}\right)$ não depende de $\alpha_{i}$, e, portanto, é uma constante que necessita ser calculada uma única vez e apenas as diagonais de $Z_{i}^{\prime} M Z_{i}$ necessitam ser calculadas em cada iteração.

VanRADEN (1986) propôs a forma quadrática $w_{i}^{\prime} \hat{u}_{i}$ em que $w_{i}=D_{i} Z_{i}^{\prime} M y$

e $D_{i}=\operatorname{diag}\left(Z_{i}^{\prime} M Z_{i}+I \alpha_{i}\right)^{-1}$. Neste caso, a falsa esperança é dada por:

$$
\begin{gathered}
E\left[w_{i}^{\prime} \hat{u}_{i}\right]=\operatorname{Tr}\left(D_{i} Z_{i}^{\prime} M Z_{i}\right) \sigma_{i}^{2} \\
\Rightarrow \hat{\sigma}_{i}^{2}=\frac{w_{i}^{\prime} \hat{u}_{i}}{\operatorname{Tr}\left(D_{i} Z_{i}^{\prime}\right.} \overline{\left.M Z_{i}\right)} .
\end{gathered}
$$

Desde que $D_{i}$ é diagonal, então $\operatorname{Tr}\left(D_{i} Z_{i}^{\prime} M Z_{i}\right)$ é facilmente obtido, mas, no caso, depende de $\alpha_{i}$ e tem de ser computado a cada iteração.

\subsubsection{Método da Verossimilhança Integrada - VEIL}

O método foi proposto por GIANOLA \& FOULLEY (1990) e baseia-se em métodos Bayesianos. Através deste método, os componentes de covariancia são obtidos a partir de verossimilhanças integradas.

Pelo método da máxima verossimilhança, as fórmulas são derivadas a partir da função de verossimilhança de $y$ (ou do logaritmo dela). No método REML, fórmulas são derivadas a partir da função de verossimilhança de $L^{\prime} y$, um vetor de contrastes de erros. Na terminologia Bayesiana, ML é derivada da maximização da densidade conjunta "a posteriori" de $\beta$, os efeitos fixos e dos componentes de covariancia dado $y$, isto é:

$$
p(\beta, V / y) \propto p(y / \beta, V)
$$

Se esta densidade conjunta for integrada em relação a $\beta$ e em seguida a função de verossimilhança marginal for maximizada em relação aos componentes de covariancia, os estimadores resultantes serão estimadores REML.

$\mathrm{O}$ método VEIL utiliza o mesmo conceito, um passo a frente, integrando a verossimilhança marginal em relação a cada componente de matriz $V$, assumindo que outros valores destes componentes de variancia tenham sido estimados (SCHAEFFER, 1993). As fórmulas resultantes são similares àquelas obtidas pelos métodos REML e ML, 
a diferença básica é que VEIL considera os graus de liberdade utilizados para estimar $\beta$ e cada um dos componentes de covariancia. Para o caso univariado, tem-se:

$$
\hat{\alpha}_{i}=\frac{\hat{\sigma}_{0}^{2}}{\left(\hat{u}_{i}^{\prime} \hat{u}_{i}+\hat{\sigma}_{0}^{2} \operatorname{Tr}\left(c^{i i}\right) /\left(q_{i}-4\right)\right)} .
$$

$\mathrm{E}$, considerando-se parentesco entre individuos, tem-se

$$
\hat{\alpha}_{i}=\frac{\hat{\sigma}_{0}^{2}}{\left(\hat{u}_{i}^{\prime} A^{-1} \hat{u}_{i}+\hat{\sigma}_{0}^{2} \operatorname{Tr}\left(c^{i i} A_{i}^{-1}\right) /\left(q_{i}-4\right)\right)} .
$$

Assim,

$$
\hat{\sigma}_{0}^{2}=\frac{\left(y^{\prime} y-\hat{\theta}^{\prime} W^{\prime} y\right)}{(N-p-2 c)} \quad \text { e } \quad \hat{\sigma}_{i}^{2}=\frac{\hat{\sigma}_{0}^{2}}{\hat{\alpha}_{i}} .
$$

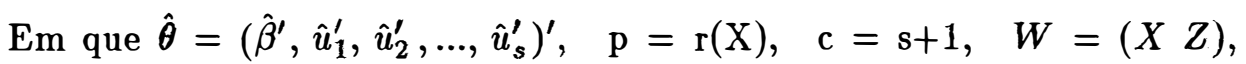
$q=\sum_{i=1}^{s} q_{i}$. Além disso, $c^{i i}$ é a partição de $\left(W^{\prime} W+\Sigma\right)^{-1}$, correspondente aos efeitos de $u_{i}$, sendo

$$
\Sigma=\left[\begin{array}{ll}
0 & 0 \\
0 & T^{-1}
\end{array}\right] \text { com } T^{-1}=\bigoplus_{i=1}^{s} A_{i}^{-1} \frac{\sigma_{0}^{2}}{\sigma_{i}^{2}} .
$$

O método é iterativo, da mesma forma que REML e ML. Note que cada $q_{i}$ tem de ser maior do que 4 e $\mathrm{N}$ tem de ser maior do que $(p+2 c)$.

\section{Comentários Adicionais}

Os métodos de estimação de componentes de covariância apresentados são aqueles comumente utilizados na área de melhoramento animal. Cada um desses métodos apresenta propriedades que, em determinada situação, faz com que um ou outro seja de uso mais recomendado. O método REML tem sido considerado (SEARLE, 1971; HARVILLE, 1977; HENDERSON, 1984a; MANDOUR et al., 1985; MEYER, 1986; LIN, 1987; VALENTE, 1988; GROENEVELD \& KOVAC, 1990; MISZTAL, 1990; BOLDMAN \& VAN VLECK, 1991; KEELE et al., 1991; MEYER, 1991 e 1993) e CALVIN, 1993, entre outros) o preferido para estimar componentes de covariancia de dados não balanceados. As razões para esta preferencia passam pelas propriedades estatísticas desejáveis que os estimadores REML apresentam, a maioria das quais comuns com o método ML. Algumas dessas propriedades, segundo HARVILLE (1977) são:

- i) Os estimadores são funções de estatísticas suficientes, consistentes e assintoticamente normais e eficientes;

- ii) Aproximações são sempre bem definidas;

- iii) Restrições para não negatividade dos componentes de variância ou dos autovalores das matrizes de variância e covariância, ou outras restrições no espaço de parâmetros, não causam dificuldades conceituais na aplicação do método; 
- iv) Soluções são quase sempre no espaço de parâmetros;

- v) Variâncias amostrais e erro quadrático médio menores do que aquelas obtidas por outros métodos que produzem estimadores não viciados.

- vi) Outra importante propriedade, especialmente para aplicações na área de melhoramento, é que o método REML reduz ou elimina o viés de pequenas amostras e/ou decorrente da seleção de animais (MEYER, 1983 e 1989).

VALENTE (1988), nos Estados Unidos, confirma a maioria das propriedades mencionadas. Já VALÉRIO FILHO (1991), no Brasil, prova, através de dados simulados, a grande eficiência do método REML, especialmente para dados desbalanceados, comparados aos métodos MIVQUE0, ANOVA e ML.

No passado REML foi pouco utilizado na prática, por ser um método que, quando aplicado na forma convencional, demanda alta capacidade de memória RAM (Random Access Memory) e velocidade dos recursos computacionais (VALENTE, 1988, MISZTAL, 1990, MEYER, 1993, entre outros). Todavia, o avanço considerável que se tem observado na área de informática, bem como a adaptação e implementação de eficientes algorítmos computacionais, têm permitido um uso generalizado deste método na área de melhoramento. Assim, nos últimos anos, inúmeros trabalhos de pesquisa, utilizando o método REML, para estimação de covariância, foram publicados (veja, entre outros, SMITH \& GRASER, 1986; MEYER, 1983, 1985, 1986, 1988, 1989, 1991 e 1993; MEYER et al., 1991, LIN \& LEE, 1986; GRASER et al., 1987; LAIRD et al., 1987; VALENTE, 1988; DA et al., 1989; BOLDMAN \& VAN VLECK, 1991; GROENEVELD \& KOVAC, 1990; CHAUHAN \& HAYES, 1991; KEELE, 1991; WEIGEL et al., 1991, MISZTAL, 1992 e VAN VLECK \& GREGORY, 1992).

Estimação de componentes de variância pelo método REML teve ińrcio pelo desenvolvimento de modelos espećfficos de análise de variância (ANOVA) balanceados. CUNNINGHAN \& HENDERSON (1968) desenvolveram um procedimento iterativo para estimação de variâncias e efeitos fixos. THOMPSON (1968) corrigiu o erro no denominador do estimador da variancia residual obtida por Cunninghan \& Henderson. Posterioremente, PATHERSON \& THOMPSON (1971) desenvolveram a metodologia REML para o caso de dados não balanceados e modelos univariados. Maximizaram a parte da verossimilhança da função densidade de probabilidade da distribuição normal associada com os efeitos aleatórios, após ajustá-los para as estimativas dos efeitos fixos. A derivação foi para uma função de $y, L^{\prime} y$ tal que $\left(L^{\prime} y\right)=\left(L^{\prime} X \beta\right)$, com a restrição $L^{\prime} X=\emptyset$. Assim, os estimadores dos componentes de covariância que maximizam a verossimilhança são invariantes à restrição na obtenção de $\hat{\beta}$.

Posteriormente, THOMPSON (1973) estendeu a metodologia para modelos multivariados para o caso especial de igualdade de matriz de delineamento. Esta restrição foi removida por SCHAEFFER et al. (1978) para o caso de covariancia residual nula entre características.

Recentemente, vários algorftmos foram desenvolvidos, os quais poderiam ser considerados para computar ML ou REML (VALENTE, 1988). Um desses algorftmos usa a iteração sob a equação MIVQUE até a convergência, com a condição que a 
convegência ocorra no espaço de parâmetros (HARVILLE, 1977; HENDERSON, 1984a e SCHAEFFER, 1993). Um algoritmo similar é aquele que utiliza a mesma forma quadrática como para o método MIVQUE, mas as esperanças destas formas quadráticas são calculadas como se o valor prévio ou preliminar fosse o verdadeiro valor (SCHAEFFER, 1993, método da falsa esperança). A vantagem deste algoritmo é que as estimativas permanecem no espaço de parâmetro e as esperanças das formas quadráticas são mais simples de serem obtidas do que aquelas calculadas pelo método MIVQUE, mas a convergência é mais lenta.

MEYER (1983) apresentou um algoritmo para estimação de componentes de covariância baseado no método "Scorring" de Fisher (MSC) para modelos mistos, com um único efeito aleatório, além do erro, tipo modelo de reprodutor ("Sire Model"). Em contraste às aplicações anteriores do método REML por SCHAEFFER et al. (1978), o procedimento acomoda covariancia de ambiente entre caracteristicas e também permite análise de diferentes números de características medidas em diferentes indivíduos.

MEYER (1985) propôs outro algorítmo cujo procedimento envolve uma transformação para a escala canônica, reduzindo uma análise de q-características para uma outra correspondendo à $\mathrm{q}$ análises univariadas. A relação entre indivíduos é ignorada e a convergencia é rápida, mas não se pode garantir que as estimativas da matriz de covariâncias estejam no espaço de parâmetros. Neste caso, em análises de características múltiplas altamente correlacionadas, existe uma forte chance que, devido á amostragem, a matriz de covariancia de componentes de individuos (por exemplo, reprodutor) não seja positiva definida. A probabilidade de que isto ocorra aumenta com o número de variáveis (HILL \& THOMPSON, 1978).

SCHAEFFER (1986) apresenta dois métodos (CANMT - Canonical Transformation in Multiple Trait Procedure e TRIMT - Triangular Transformation in Multiple Trait Procedure) que são computacionalmente simples para estimação de variâncias e covariancias, os quais garantem estimativas no espaço de parâmetros. O primeiro método é similar ao apresentado por MEYER (1985) e é restrito à situação em que cada indivíduo é observado para todas q características. $\mathrm{O}$ segundo método é menos restritivo do que o primeiro e conseqủentemente a demanda computacional é maior. Permite a inclusão de mais do que um fator aleatório no modelo e aceita valores perdidos para algumas características, mas é assumido o mesmo modelo para todas características.

Um outro algoritmo foi proposto por MEYER (1986) para modelos que incluam um fator aleatório além do erro ("Sire Model", por exemplo) para aumentar a rapidez de convergência comparada ao algoritmo EM, como também para reduzir o tempo gasto por iteração, comparado ao método "Scoring" de Fisher (MSC). Este algoritmo foi denominado "Short Cut" (SHC), que combina características do algorftmo EM, para estimar os componentes dentro de classes do fator aleatório (erro) com o msc para estimar os componentes entre classes do fator aleatório. $O$ procedimento pode ser utilizado para estimar componentes de covariância em um modelo misto multivariado, quando existem valores perdidos para algumas características. Uma desvantagem desse método é que ele não garante a obtenção de estimativas dentro do espaço de parâmetros, embora seja bastante robusto quanto a isto. VALENTE (1988) comparou este algoritmo com duas formas do algoritmo EM. Foram utilizados sete conjuntos de dados simulados, sob uma ampla 
variedade de condições, e um conjunto de dados reais, na estimação de componentes de covariância de características múltiplas. Adotou-se um um modelo de reprodutor e valores perdidos para algumas características. Verificou-se que o algoritmo SHC convergiu uniformemente mais rápido do que as duas versões do algoritmo EM e as estimativas dos componentes de covariancia foram, em geral as mesmas. A desvantagem deste procedimento é que ele não foi adaptado para o caso de modelos mais complexos, com vários tipos de efeitos fixos e mais de um fator aleatório além do erro. Para estes casos, parece que o método tem sua aplicação dificultada.

Finalmente, SMITH \& GRASER (1986) e GRASER et al. (1987) propuseram não tomar derivadas ou esperanças. Através de um procedimento livre de derivadas, em análise univariada, derivaram um algoritmo para estimar as variancias genéticas aditivas e do erro, sob um modelo animal. Explorando a estrutura esparsa da matriz de coeficientes, mostraram que o procedimento é apropriado para dados de grandes experimentos de seleção envolvendo milhares de animais. $O$ algoritmo apresentado parece ter aberto uma grande perspectiva para um uso mais acentuado do método da máxima verossimilhança restrita (REML).

$\mathrm{O}$ método livre de derivadas simplesmente tenta encontrar diferentes $R$ e $G$, matrizes de covariância do erro e dos fatores aleatórios, respectivamente, até que a combinação que maximiza o logaritmo da função de verossimilhança $\left(L_{2}\right)$ seja encontrada. A aproximação não é computacionalmente tão rápida, mas talvez mais simples do que aproximações tipo EM-REML, envolvendo derivadas, esperanças e inversas. VAN VLECK (1992, páginas 13 a 15) apresenta um exemplo prático e simples para facilitar a compreensão do método.

Baseando-se em (25), (pág. 48), e sabendo-se que os termos $\log |C|$ e $y^{\prime} P y$ são aqueles que realmente apresentam maior dificuldade computacional para avaliar $L_{2}$, SMITH \& GRASER (1986) propõem a eliminação de Gauss (GE), que é freqủentemente usada para obtenção de soluções de conjuntos de equações lineares. MEYER $(1989,1991$ e 1993) incorpora essas idéias em uma notável série de programas denominados DFREML ("Derivarite Free Restricted Maximum Likelihood"), os quais aproveitam a vantagem de a matriz de coeficientes ser uma matriz esparsa e desenvolve rotinas que trabalham com a técnica de lista encadeada. GE é mais eficiente do que a inversão para obtenção de soluções para matrizes esparsas. GE é geralmente muito mais eficiente em termos de requerimento de memória e tempo de processamento (VAN VLECK, 1992). Os programas incluem cálculo da matriz $A^{-1}$, a inversa dos coeficientes de parentesco entre animais. A estratégia de procura para atualizar $R$ e $G$ para as equações do modelo misto (MME) é através do método Simplex (politopo) ou do método de Powell ou método das direções conjugadas (veja (2.1.5.1.2) e (2.1.5.1.3)), que são métodos eficientes para otimização não linear.

Os programas DFREML expandem por um fator de 5 a 10 o número de equações que anteriormente poderiam ser manipuladas com a estimação REML e reduzem o tempo de computação para estimação de componentes de variancia por fator ainda maior. A convergência usualmente ocorre com menos cálculo da função de verossimilhança do que o número de inversões necessárias no algorítmo EM. No entanto, para superfícies muito planas, podem ocorrer problemas de convegência. 
Após a criação e posterior adaptação do algoritmo livre de derivadas, BOLDMAN \& VAN VLECK (1991) criam um novo algorftmo para obtenção dos componentes de covariância em análise uni ou multivariada. Adaptam a técnica de fatoração ou decomposição de Cholesky, acoplada ao conjunto de rotinas para solução de problemas para matrizes esparsas, denominado SPARSPAK (GEORGE et al., 1980). Aproveitando programas já escritos e testados por MEYER (1988), escrevem novos programas. Incorporam aos programas o conjunto de rotinas da SPARSPAK, desenvolvm rotinas especificas para imposição de restrições aos parâmetros relativos aos efeitos fixos e montam o programa denominado MTDFREML ("Multiple Trait Derivative Free Restrcted Maximum Likelihood"). Com esses desenvolvimentos, há uma redução drástica do tempo de processamento com o uso de rotinas da Sparspak-Cholesky em comparação com a eliminação de Gauss original, acoplada a lista encadeada de MEYER (1988). Seguindo a mesma tendencia, visando obter programas mais eficientes, MEYER (1993) adiciona novas opções ao sistema DFREML. Incorpa rotinas de operação com matrizes esparsas tipo SPARSPAK (GEORGE et al. 1981) acopladas à decomposição de Cholesky da matriz de coeficientes $(C)$.

\subsection{Algoritmo para Simulação de Modelos Mistos}

A técnica de simulação de variáveis constitui uma importante ferramenta de trabalho, especialmente em estudos de caracteristicas da distribuição de probabilidades referentes às populaçãos biológicas (GODOI, 1978b). Além disso, graças ao fantástico avanço na área de informática, é cada vez mais fácil e prática a simulação de variáveis ou de amostras baseada em modelos matemáticos apropriados, com parâmetros conhecidos, com o objetivo de se verificar a adequação de determinada metodologia ou na realização de comparações entre métodos.

Outro importante uso da técnica de simulação é aquela realizada com o objetivo de se avaliar as estimativas e as variancias amostrais dos componentes de covariâncias para uma estrutura de dados particular (VAN VLECK, 1994).

A simulação de vetores multinormais está pautada no seguinte teorema, que não será demonstrado:

Seja $z \stackrel{i i d}{\sim} N(0, I)$, isto é $z_{1}, z_{2}, \ldots, z_{p} \stackrel{i i d}{\sim} N(0,1)$ e seja $\Sigma U=U \Lambda$, em que $U^{\prime} U=I$ e $\Lambda=\operatorname{diag}\left(\lambda_{1}, \lambda_{2}, \ldots, \lambda_{p}\right)$. Os $\lambda_{i}$ 's são autovalores de $\Sigma$ e $U$ é uma matriz cujas colunas são autovetores normalizados correspondentes a $\lambda$. Então, $U^{\prime} \Sigma U=\Lambda$ e $U \Lambda U^{\prime}=\Sigma, U \Lambda^{\frac{1}{2}} \Lambda^{\frac{1}{2}} U^{\prime}=\Sigma$.

Seja a transformação $y=\mu+U \Lambda^{\frac{1}{2}} z$. Como $\mathrm{E}(y)=\mu+U \Lambda^{\frac{1}{2}} 0=\mu \mathrm{e}$ $\operatorname{Var}(y)=0+U \Lambda^{\frac{1}{2}} I \Lambda^{\frac{1}{2}} U^{\prime}=\Sigma$, então, a partir de p variáveis $N(0,1)$ pode-se obter $\mathrm{p}$ variáveis $N(\mu, \Sigma)$. As variáveis normais $N(0,1)$ podem ser obtidas, por exemplo, pela transformação de Box \& Miller (BOX \& MILLER, 1958) aos pares, da seguinte forma:

$$
\left.\left.z_{i}=\sqrt{(}-2 \log \left(u_{1}\right)\right) \cos \left(2 \pi u_{2}\right) \quad \text { e } \quad z_{j}=\sqrt{(}-2 \log \left(u_{1}\right)\right) \operatorname{sen}\left(2 \pi u_{2}\right)
$$

em que $u_{1}$ e $u_{2} \sim U(0,1)$. A eficiência da simulação dependerá da eficiência da geração das variáveis uniformes. 
Além disso, a transformação integral de probabilidade, apresentada em MOOD et al. (1974), mostra que se $\mathrm{X}$ é uma variável aleatória com função de distribuição contínua $\mathrm{F}(\mathrm{x})$, então $U=\mathrm{F}(\mathrm{x})$ é uniformemente distriburda sobre o intervalo $(0,1)$. Por outro lado, se $U$ é uniformemente distriburdo sobre o intervalo $(0,1)$, então $\mathrm{x}=\mathrm{F}^{-1}(U)$ tem função de distribuição $F($.$) . Isto significa que se a função de distribuição de \mathrm{X}$ é conhecida, então valores de $\mathrm{x}$, da variável $\mathrm{X}$, com distribuição $\mathrm{F}$, poderão ser encontradas, calculando-se $\mathrm{F}^{-1}(u)$, em que $u$ representa um valor de $U$ (MACHADO, 1993).

Simulação de modelos genéticos quantitativos usualmente envolve a simulação de valores genotípicos para a geração de pais, seguido pela transmissão desses efeitos simulados para a progênie. Para modelo animal, a estrutura de dados inclui o cálculo dos coeficientes de parentesco $(A)$ entre os individuos com registros e de seus ancestrais.

A simulação de modelos mistos dependerá basicamente do tipo de modelo adotado, se ele inclui um único fator aleatório, mais de um fator aleatório, com ou sem correlação entre niveis desses fatores; da estrutura de variancia de cada um desses fatores e ainda depende se o modelo é uni ou multivariado. Nosso interesse neste trabalho é o de simular modelos em que $y$ tenha distribuição normal multivariada. Para a simulação de vetores multinormais, veja, por exemplo, os trabalhos de KINDERMAN \& RAMAGE (1976); RODDA et al. (1977); GODOI (1978a e 1978b), VAN VLECK \& GREGORY (1992) e VAN VLECK (1994).

VAN VLECK (1994) apresenta uma boa descrição de como se pode simular um modelo animal, linear multivariado, adotando-se a decomposição de Cholesky da matriz de coeficientes de parentesco entre animais $(A)$ e das matrizes de covariancias genética aditiva e de efeitos residuais. Nesse item apresenta-se um resumo daquele trabalho, incluindo-se algumas informações adicionais.

Segundo descrição de VAN VLECK (1994), a decomposição de Cholesky $L_{v}$ da matriz de variancia e covariancia $V=L_{v} L_{v}^{\prime}$ pode ser utilizada para a simulação de valores genéticos para uma população de indivíduos com matriz de parentesco $(A)$ conhecida. Assim, para simular um modelo animal, a estrutura da amostra, incluindo os individuos para os quais os dados serão simulados e os parentes desses, necessita ser conhecida previamente, para que se possa obter a matriz de coeficiente de parentesco entre os animais.

Se a variancia dos efeitos genéticos aditivos é $\sigma_{a}^{2}$ e $v_{a}$ é o vetor de ordem igual ao número de indivíduos $(\mathrm{N})$ de valores aleatórios normais padrão, então $a=L_{a} v_{a}$ são valores genéticos aditivos simulados para os $\mathrm{N}$ indivíduos. Os cálculos para acumulação dos elementos de $A$ podem ser feitos com um valor aleatório normal por vez. A simulação de características múltiplas pode ser feita de forma similar, considerando-se as propriedades do produto direto da matriz $G_{t N}$, a matriz de covariancia para as q características e $\mathrm{n}$ individuos. Com as características ordenadas por individuo, $L_{G_{q n}}=L_{A} \otimes L_{G}$, em que $L_{G}$ representa a decomposição de Cholesky da matriz $G$, a matriz de covariancias genéticas entre as características. $O$ padrão de acumulação de valores genéticos é feito seqüencialmente, com um vetor de ordem q, de valores aleatórios normais padrão, por vez. 


\subsubsection{Simulação de valores genéticos para características múltiplas sem pa- rentesco entre animais}

Imagine uma situação em que se pretenda simular valores para três caracteristicas (A, B e C). Seja

$$
\begin{gathered}
s_{g}=\left(G_{A} G_{B} G_{C}\right)^{\prime} \text { com } \\
V\left(s_{g}\right)=G=\left(\begin{array}{lll}
\sigma_{G_{A}}^{2} & \sigma_{G_{A} G_{B}} & \sigma_{G_{A} G_{C}} \\
\sigma_{G_{A} G_{B}} & \sigma_{G_{B}}^{2} & \sigma_{G_{B} G_{C}} \\
\sigma_{G_{A} G_{C}} & \sigma_{G_{B} G_{C}} & \sigma_{G_{C}}^{2}
\end{array}\right) .
\end{gathered}
$$

Decompōe-se $G=L_{g} L_{g}^{\prime}$. Para cada animal, geram-se 3 (três) variáveis com distribuição normal padrão $g=\left(\begin{array}{lll}g_{A} & g_{B} & g_{C}\end{array}\right)^{\prime}$. Então, $s_{g}=L_{G} g$.

Os efeitos de ambiente, com matriz de covariancia $E$, podem ser gerados de modo semelhante, através de um conjunto de outras 3 (três) variáveis com distribuição normal padrão, $e=\left(\begin{array}{lll}e_{A} & e_{B} & e_{C}\end{array}\right)^{\prime} \operatorname{com}, s_{e}=L_{E} e$.

\subsubsection{Simulação de valores genéticos para características múltiplas com pa- rentesco entre animais}

Será admitido que cada individuo tenha um valor genético para cada caracterfstica (se houver registros perdidos, o efeito de ambiente não será gerado para aquele registro) e que haja efeito genético entre animais parentes e efeito de ambiente entre características de um mesmo animal, mas não exista este efeito entre animais.

Nessa situação, aproveitam-se as propriedades do produto direto para (q) características de animais parentes. Dessa forma, em vez da decomposição de Cholesky ser de orden $\mathrm{qN}$, será apenas de ordem q, para a matriz de variância e covariância genética $G$, e de ordem N para a matriz de parentesto entre individuos $(A)$,

Sejam os valores genéticos aditivos ordenados por animal e $G_{q N}$ a matriz de variância e covariância genética. Então, $G_{q N}=A \otimes G$, em que $\otimes$ é o operador de produto direto. Então, pela fatoração de Cholesky

$$
\begin{aligned}
L_{G_{q N}} & =L_{A} \otimes L_{G} . \text { Note que } L_{G_{q N}} L_{G_{q N}}^{\prime}=G_{q N} \text { e que } \\
\left(L_{A} \otimes L_{G}\right)\left(L_{A} \otimes L_{G}\right)^{\prime} & =L_{A} L_{A}^{\prime} \otimes L_{G} L_{G}^{\prime}=A \otimes G .
\end{aligned}
$$

Seja um pequeno exemplo com $\mathrm{N}=3$ (três) e $\mathrm{q}=2$ (duas) características. Neste caso, tem-se:

$$
L_{A}=\left(\begin{array}{lll}
a_{11} & 0 & 0 \\
a_{21} & a_{22} & 0 \\
a_{31} & a_{32} & a_{33}
\end{array}\right) \quad e \quad L_{G}=\left(\begin{array}{ll}
g_{11} & 0 \\
g_{21} & g_{22}
\end{array}\right)
$$

Então

$$
L_{A} \otimes L_{G}=\left(\begin{array}{ccc}
a_{11} L_{G} & 0 & 0 \\
a_{21} L_{G} & a_{22} L_{G} & 0 \\
a_{31} L_{G} & a_{32} L_{G} & a_{33} L_{G}
\end{array}\right)
$$


Qualquer bloco abaixo da diagonal, $a_{i j} L_{G}$, é triangular inferior. Por exemplo:

$$
a_{21} L_{G}=\left(\begin{array}{ll}
a_{21} g_{11} & 0 \\
a_{21} g_{21} & a_{21} g_{22}
\end{array}\right)
$$

Admitindo-se um vetor de tamanho $\mathrm{qN}$ de valores aleatórios com distribuição normal (rnv), seria gerado e premultiplicado por $L_{A} \otimes L_{G}$. Seja $v_{i}$ o vetor de rnv's associado com as q características do animal i. Também seja $s_{i}$ o vetor de q valores genéticos simulados para o animal i. Então:

$$
\left(\begin{array}{l}
s_{1} \\
s_{2} \\
s_{3}
\end{array}\right)=\left(\begin{array}{l}
a_{11} L_{G} v_{1} \\
a_{21} L_{G} v_{1}+a_{22} L_{G} v_{2} \\
a_{31} L_{G} v_{1}+a_{32} L_{G} v_{2}+a_{33} L_{G} v_{3}
\end{array}\right)
$$

Os termos $L_{A}$ tornam-se multiplicadores escalares dos vetores obtidos de $L_{G} v_{i}$. O padrão é tal que $s$ pode ser acumulado facilmente de $v_{i}$, sem necessidade de manter em memória o vetor $v$. Um vetor de efeitos de ambiente correlacionados $s_{e}$ pode ser adicionado a $s_{i}$, a fim de se obter o vetor de efeitos fenotipicos, em que $s_{e}=L_{E} e_{i}$ e $e_{i}$ é um vetor de q valores aleatórios com distribuição normal, gerados para o animal i.

Seja $p(i, j)$ o vetor de valores fenotípicos da característica j para o animal i e $L_{G}, L_{E}$ e $L_{A}$ as decomposições de Cholesky das matrizes $G, E$ e $A$, respectivamente. Então, $p(i, j)=L_{G} \otimes v_{G} \otimes L_{A}+L_{E} \otimes v_{e}$. No apêndice 1 é apresentado um esquema do programa em linguagem FORTRAN para simulação de vetores multinormais.

\subsubsection{Simulação de valores genéticos e efeitos correlacionados para caracterís- ticas múltiplas com parentesco entre animais}

Efeito direto e efeitos correlacionados são simulados de forma similar ao apresentado anteriormente. A diferença é que agora a matriz $G$ apresenta uma outra composição, isto é,

$$
G=\left(\begin{array}{cc}
\sigma_{a}^{2} & \sigma_{a m} \\
\sigma_{a m} & \sigma_{m}^{2}
\end{array}\right)
$$

em que $\sigma_{a}^{2}$ e $\sigma_{m}^{2}$ são as variâncias dos efeitos genéticos diretos e correlacionados e $\sigma_{a m}$ é a covariancia entre estes efeitos. 0 restante do processo segue a mesma linha apresentada no item anterior e no apêndice 1 . 


\section{MATERIAL E MÉTODOS}

O material objeto deste estudo compõe-se dos sistemas (DFREML, MTC e MTDFREML) que serão avaliados e dos dados para análise.

\subsection{Descrição dos Sistemas DFREML, MTC e MTDFREML}

\subsubsection{SISTEMA DFREML (Derivative Free Restricted Maximum Likelihood)}

O sistema DFREML (Método da Máxima Verossimilhança Restrita utilizando o procedimento Livre de Derivadas) consiste de um grande conjunto de programas escrito por MEYER (1988, 1989, 1991 e 1993) em linguagem FORTRAN padrão (F77), montados sob uma plataforma UNIX. Para que sejam processados utilizando o sistema operacional MS-Dos ou compatfvel, há necessidade de fazerem-se pequenas adaptações nos programas e utilizar compiladores apropriados. Os programas consistem de um amplo conjunto de rotinas especfficas ou gerais que são adotadas nos programas principais do sistema DFREML versão 2.1.09. Após a compilação do sistema, obtêm-se quatro programas descritos na tabela 1.

O propósito principal do sistema DFREML é o cálculo de componentes de (co)variancia, para variáveis contínuas, com distribuição multinormal, adotando-se o método REML. Todavia, com a mesma flexibilidade e facilidade operacional, é possfvel obterem-se as solução para efeitos fixos e aleatórios ajustados (incluindo variáveis classificatórias ou contínuas) e cálculo de erros amostrais e regióes de confiança para parâmetros individuais, utilizando a técnica de aproximação univariada, pelo ajuste de perfil de verossimilhança.

Os programas DFUNI, DFMUV, bem como o DFMUW, permitem o ajuste de modelos mistos com qualquer número de efeitos fixos e aleatórios, de acordo com o limite de memória RAM ("Random Access Memory") disponfvel (os valores máximos são definidos em arquivo de parametro apropriado para cada um dos programas). Admite-se a especificação de efeitos aleatórios além do erro, devido a animal, efeito aleatório adicional correlacionado com animal e efeito aleatório não correlacionado. $\mathrm{O}$ sistema apresenta um manual do usuário bem descrito, facilitando a instalação e operação.

Para realizar uma análise utilizando-se o sistema DFREML, há necessidade de dois arquivos. $\mathrm{O}$ arquivo de pedigree e o de dados (em análise univariada o arquivo de 
Tabela 1. Programas do sistema DFREML, versão 2.1.09

\begin{tabular}{ll}
\hline Programa & Propósito \\
\hline DFPREP & $\begin{array}{l}\text { Preparação dos dados: Recodifica efeitos fixos e aleató- } \\
\text { rios em ordem crescente e calcula a matriz de parentesco } \\
\text { entre individuos (animais). }\end{array}$ \\
DFUNI & $\begin{array}{l}\text { Executa análise univariada ou análise multivariada para } \\
\text { modelos de análise para todas características e explora } \\
\text { igualdade de matriz delineamento (se aplicável). }\end{array}$ \\
DFMUV & $\begin{array}{l}\text { Executa análise multivariada com um ou mais fatores } \\
\text { aleatórios além do erro, com o mesmo modelo de análise } \\
\text { para todas caracteristicas, explorando a igualdade de } \\
\text { matriz delineamento (se aplicável). }\end{array}$ \\
DFMUW & $\begin{array}{l}\text { Executa análise multivariada com um ou mais fatores } \\
\text { aleatórios além do erro e permite o ajuste de diferen- } \\
\text { tes modelos para diferentes características e covarian- } \\
\text { cias não estimáveis. }\end{array}$ \\
\hline
\end{tabular}

pedigree pode ser o próprio arquivo de dados). Em seguida, deve-se executar o programa DFPREP, cuja sequiencia de operação é auto explicativa. Para processar um dos programas DFUNI, DFMUV ou DFMUW existem nove opções disponfveis ao usuário. Essas opcões são apresentadas na tabela 2 .

A opção -2, da Tabela 2, presente nos programas DFUNI, DFMUV e DFMUW, é executada com o objetivo de aumentar a velocidade de processamento e otimização no uso de memória RAM. É possivel realizar o reordenamento e fatoração das equações com vistas a reduzir ao minimo o numero de elementos não nulos gerados durante o processo de ordenação e fatoração das MME. É importante entender como são organizadas as equações, para se escolher adequadamente a melhor opção.

Quando se define o modelo a ser analisado (opção -1), o sistema numera as equaçōes na seguinte ordem:

a) Uma linha para cada termo do lado direito das MME;

b) Uma linha para cada coeficiente de regressão ajustado;

c) Uma linha para cada nfvel de efeito fixo (para análise multivariada, os níveis são ordenados dentro de efeito fixo, classificado por característica);

d) Uma linha para cada caracteristica e nível de efeito aleatório adicional (para análise multivariada, esses são ordenados conforme as características dentro de níveis);

e) Uma linha para cada classe de animal ajustado e característica, ordenados conforme característica dentro de efeito.

Assim, o usuário pode escolher até quatro op̧̧ões de reordenamento (todas 
Tabela 2. Resumo das opções dos programas DFREML V. 2.1.09

\begin{aligned} & \hline Opção Propósito \\ & \hline-1 Definição do modelo \\ &-2 Opção para reordenar equações (opcional) \\ & 0 Iníio do processo de estimação \\ & 1 Continuação do processo de estimação \\ & 2 Reinicia a estimação ("ReStart") \\ & 3 Remaximiza a verossimilhança fixando parâmetros \\ & 4 Avalia um ponto individual de Log $\left(L_{2}\right) \\ & 5$ Obtém solução para efeitos fixos e aleatórios, assumindo que \\ & as (co)variâncias estimadas são as verdadeiras \\ & 6 Como a opção 5, mas os componentes de covariâncias são lidos \\ & 7 Aproxima o perfil de verossimilhança para um dado parâmetro \\ & e deriva variância amostral e intervalo de confiança para ele \\ & 8 Seleciona o vetor com maior verossimilhança e imprime o resu- \\ & mo dos resultados \\ & 9 Imprime as dimensões dos vetores e matrizes definidos no ar- \\ & quivo de parâmetros \\ & \hline\end{aligned}

inclusas na opção -2). Na primeira opção não se faz qualquer tipo de ordenação. Neste caso, é feito o processamento sequêncial das equações, absorvendo uma linha (e coluna) por vez, começando da última linha para a primeira. Essa ordem de solução garante (GRASER et al. 1987), que os dados de animais mais novos sejam processados em primeiro lugar e os dos mais velhos por último. Com isso reduz-se ao minimo o número de elementos não nulos gerados durante o processo de absorção. Na segunda opção, o próprio usuário especifica a ordem de interesse. Neste caso, o programa deve ler as informações de um arquivo. Na terceira opção, o programa faz um reordenamento das equações, partindo-se $\mathrm{da}(\mathrm{s})$ linha(s) com menor para a(s) linha(s) com maior número de elementos não nulos. Se houver empate, a linha de ordem de equação maior é colocada em primeiro lugar. A escolha de qualquer uma dessas três opções implica em usar a eliminação de Gauss e lista encadeada na obtenção de $y^{\prime} P y$ e $\log |C|$. Finalmente, na $4^{\underline{a}}$ opção, faz-se uma ordenação seguida de fatoração. Nesse caso, adota-se o algoritmo de minimo grau ("minimum degree algorithm") (GEORGE \& LIU, 1981), no qual são estabelecidas permutações da matriz original, seguidas por uma fatoração, estabelecendo uma estrutura da matriz fatorada e adotando-se um esquema comprimido de armazenamento para os elementos da matriz esparsa (MEYER, 1993, página 30). A escolha dessa opção, implica no uso do processo de decomposição das MME, utilizando as rotinas SPARSPAK. Após os processos de ordenação e fatoração terem sido realizados, a procura do máximo da função de verossimilhança restrita é feita adotando-se o método simplex ou o método de Powell, os quais procuram iterativamente o mínimo de $-2 \log \left(\mathrm{L}_{2}\right)$ até a convergência. Essa será considerada alcançada quando a variancia de $-2 \log \left(\mathrm{L}_{2}\right)$ for menor do que um valor especificado pelo usuário (o valor padrão normalmente utilizado por Meyer é $10^{-8}$ ).

Operacionalmente, na estimação dos componentes de variancia e covariância, os programas DFUNI, DFMUV ou DFMUW oferecem 3 (três) opções: Iniciar 
(opção 0), continuar (opção 1) ou repetir (opção 2). As opções 0 e 2 sempre devem ser utilizadas, uma vez que, adotando-se o método livre de derivadas, não se tem garantia de que as soluções obtidas em um primeiro passo sejam aquelas que correspondam ao máximo da função de verossimilhança. Assim, utilizando-se os componentes previamente estimados, pode-se repetir o processo para verificar se o máximo encontrado corresponde a um máximo local ou ao máximo global da função de verossimilhança (veja seção (3.1.4), pág. 68).

\subsubsection{SISTEMA MTC ("Multitrait REML Estimation")}

MISZTAL (1993) desenvolve uma série de programas em linguagem FORTRAN padrão (F77), objetivando estimar componentes de covariância e soluções para efeitos fixos e fatores aleatórios, na classe de modelos mistos. A tabela 3 descreve o propósito de cada programa. Para nosso interesse são utilizados apenas os programas RENUM e MTC, os quais serão denominados neste trabalho de sistema MTC.

O programa RENUM renumera efeitos classificatórios, fixos e aleatórios, e remove animais redundantes no modelo, além de checar se as progênies nasceram após os pais e cria o grupo de pais desconhecidos. O programa depende dos arquivos de "pedigree" e dos dados e, ao final de sua execução, cria o arquivo RENUM.JAA, que é o arquivo de parâmetros necessários ao programa principal. $\mathrm{O}$ arquivo de parâmetros não inclui efeito aleatório adicional e nem covariáveis. Em casos em que tais efeitos estejam presentes no modelo, o usuário deve editar o arquivo RENUM.JAA e incluf-los nas linhas apropriadas. Havendo dúvida quanto ao número de classes dos efeitos classificatórios, pode-se verificar o conteúdo do arquivo RENUM.MSG, que fornece informações adicionais a respeito de número de classes dos efeitos que foram renumerados (maiores detalhes no manual do usuário).

\section{Limitações do Sistema}

a) Não comporta efeito aleatório adicional correlacionado com o efeito de animal (efeito materno, por exemplo).

b) Não comporta covariancia de ordem maior do que 1, mas pode-se constru1-la.

c) Não considera endogamia.

d) Em análise multivariada, aplica-se apenas em casos de igualdade de matriz de delineamento entre características. 
Tabela 3. Programas do sistema MTC versão 2.0

\begin{tabular}{ll}
\hline Programa & Propósito \\
\hline JAA & $\begin{array}{l}\text { Cálculo de solução para uma classe de modelos mistos. } \\
\text { Suporta modelos cruzados com efeitos fixos e aleatórios. }\end{array}$ \\
JAA20 & $\begin{array}{l}\text { Como o JAA, mas suporta covariáveis e calcula variância do } \\
\text { erro de predição. }\end{array}$ \\
JSPFS & $\begin{array}{l}\text { Estima componentes de variância em modelos lineares mis- } \\
\text { tos univariados, utilizando o algorítmo EM, com aceleração } \\
\text { de Aitken e inversão de matrizes de classificação cruzada } \\
\text { (incluindo covariáveis). }\end{array}$ \\
MTDFS & $\begin{array}{l}\text { Como o JSPFS para característica múltipla. Utiliza trans- } \\
\text { formação canônica e é restrito a modelos com um único fa- } \\
\text { tor aleatório, além do erro. }\end{array}$ \\
MTC & $\begin{array}{l}\text { Como o MTDFS, mas com estensão para vários efeitos } \\
\text { aleatórios (não ajusta efeito aleatório adicional correlacio- } \\
\text { nado com o efeito genético aditivo). }\end{array}$ \\
RENUM & $\begin{array}{l}\text { Renumera efeitos consecutivamente, elimina redundância e } \\
\text { cria o grupo de pais desconhecidos. }\end{array}$ \\
\hline
\end{tabular}

O programa MTC requer o arquivo de parámetros gerados pelo programa RENUM e calcula componentes de covariancia, adotando-se o algorftmo EM, e a transformação canónica do vetor de valores observados. Assim, o programa requer que sejam observados valores para todas q características analisadas. A partir da obtenção das variáveis canônicas a inversão da matriz de coeficientes é realizada através da técnica de inversão de matrizes esparsas (veja (41)), adotando-se as rotinas FSPAK (PEREZ-ENCIZO \& MISZTAL, 1992). Além disso, adota-se, para obtenção dos autovalores das matrizes envolvidas em cada iteração, o processo de Gauss-Seidel. O Programa é de execução simples. Requer, ao ser executado, o nome do arquivo de parâmetros e o número de iterações que devem ser executadas. Ao final do processo, à convergência, deve-se fornecer número de iterações igual a 0 (zero). Segundo o autor, a convergencia pode ser considerada atingida quando as variáveis convG e convR forem da ordem de $10^{-6}$ ou menor. Essas variáveis representam o desvio ao quadrado entre os valores dos componentes de (co)variancia estimados entre as iterações i e i-1, para os efeitos genéticos aditivos, efeito permanente de meio (quando houver) e efeitos residuais, respectivamente. O programa MTC adota o conjunto de rotinas FSPAK, desenvolvido pelo próprio autor e colaboradores, para inversão da matriz de coeficientes, por operação de matrizes esparsas. O sistema é acompanhado de manual do usuário simples, mas suficiente para um bom acompanhamento e execução, com pequena orientação. 


\subsubsection{SISTEMA MTDFREML (Multiple Trait Derivative Free Restricted Maximum Likelihood)}

Os programas do sistema MTDFREML (Estimação pelo Método da Máxima Verossimilhança Restrita adotando-se o procedimento Livre de Derivadas (DF)) foram desenvolvidos e/ou adaptados por BOLDMAN et al. (1993). Apresentam uma boa documentação e são de fácil utilização. O sistema após compilado é composto por 3 (três) programas, descritos na tabela 4 .

Tabela 4. Programas do sistema MTDFREML

\begin{tabular}{|c|c|}
\hline Programa & Propósito \\
\hline MTDFNRM & $\begin{array}{l}\text { Programa principal para cálculo de } A^{-1} \text { e recodificação } \\
\text { para número do animal, pai e mãe (ou pai, pai do pai e } \\
\text { avó-materna). }\end{array}$ \\
\hline MTDFPREP & $\begin{array}{l}\text { Lê o arquivo de pedigree, recodifica animais e niveis de } \\
\text { efeitos fixos e fatores aleatórios. }\end{array}$ \\
\hline MTDFRUN & $\begin{array}{l}\text { Utiliza os dados preparados pelos programas anteriores e } \\
\text { calcula componentes de (co)variancia pelo máximo do } \\
\text { logaritmo da função de verossimilhança, com um algo- } \\
\text { ritmo DFREML. Adota as rotinas da SPARSPAK da Uni- } \\
\text { versidade de Waterloo (requer licença especial de uso) e } \\
\text { obtém solução para as MME,solução para variância amos- } \\
\text { tral ou ambas. } \\
\text { Em setembro de } 1994 \text { foi liberada uma nova versão do sis- } \\
\text { tema, incluindo as rotinas da FSPAK, tornando o sistema } \\
\text { totalmente de domínio público. }\end{array}$ \\
\hline
\end{tabular}

MTDFREML compõe-se de um conjunto de programas para estimar componentes de covariância, utilizando modelo animal e DFREML. Pode-se também analisar dados com modelo de reprodutor ou modelos em que não há informação de parentesco entre os individuos (arquivo de pedigree com número zero para pai e para mãe). Esses programas podem ser utilizados para análise uni ou multivariada, com modelo animal com ou sem registros repetidos por indivíduo. Solução para efeitos fixos e valores genéticos, variância amostral das soluções, contrastes e esperanças das soluções podem também ser obtidas. O programa MTDFRUN, parte do sistema, é aquele que executa as anślises e admite modelos bem gerais, inclusive diferentes modelos para cada característica em caso de análise multivariada. O tamanho da análise que pode ser executada depende do número de características, classes de efeitos fixos e de animais na análise e da capacidade de memória RAM do equipamento de processamento de dados em uso. Esse programa equivale ao programa DFMUW, componente de sistema DFREML.

Os programas foram desenvolvidos para microcomputador da linha $\mathrm{PC}$, em linguagem FORTRAN (F77). 
O procedimento adota reordenamento e fatoração das equações de modelo misto multivariado e operação de matrizes esparsas, através do conjunto de rotinas SPARSPAK e, no processo de procura do mínimo de $-2 \log \left(\mathrm{L}_{2}\right)$, é adotado o método simplex (politopo), com "step-size" de $20 \%$.

A tabela 5 apresenta um resumo com algumas caracteristicas dos sistemas DFREML, MTC e MTDFREML

Tabela 5. Características dos sistemas DFREML, MTC e MTDFREML

\begin{tabular}{|c|c|c|c|}
\hline I TEM & DFREML & MTC & MTDFREML \\
\hline Autor(es) & Karin Meyer & Ignacy Misztal & Boldman e outros \\
\hline Método de Estimação & REML & REML & REML \\
\hline Linguagem & FORTRAN & FORTRAN & FORTRAN \\
\hline \multicolumn{4}{|l|}{ Plataforma } \\
\hline Padrão & UNIX $^{1}$ & $\mathrm{DOS}^{2}$ & $\mathrm{DOS}^{2}$ \\
\hline Modelo Analisado & Animal & Animal $^{3}$ & Animal $^{3}$ \\
\hline Dados Analisados & Geral & $\begin{array}{l}\text { Igual Matriz } \\
\text { delineaneamento }\end{array}$ & Geral \\
\hline \multicolumn{2}{|l|}{ Procedimento de Pro- } & $\mathrm{EM}^{5}$ & $\mathrm{DF}^{4}$ \\
\hline $\begin{array}{l}\text { Estratégia de Procu- } \\
\text { ra do máximo de } L_{2} \\
\text { No. Máximo de }\end{array}$ & $\begin{array}{l}\text { Simplex / } \\
\text { Powell }\end{array}$ & EM & $\begin{array}{l}\text { Método } \\
\text { Simplex }\end{array}$ \\
\hline Caracteristicas & 9 & $q$ & $q$ \\
\hline No. Ef. Aleatórios & $\mathrm{r}$ & $r$ & $\mathrm{r}$ \\
\hline $\begin{array}{l}\text { Ajusta Efeitos Alea- } \\
\text { tórios adicionais }\end{array}$ & Ajusta Efeitos Alea- & $\operatorname{Sim}^{6}$ & Sim \\
\hline No. Ef. Fixos & s & $\mathrm{s}$ & s \\
\hline Aceita Valores Perdidos & Sim & Não & Sim \\
\hline Considera endogamia & Sim & Não & Sim \\
\hline \multicolumn{3}{|l|}{ Rotinas para Ma- } & SPARSPAK e FSPAK \\
\hline \multicolumn{4}{|c|}{ - Marca registrada da AT\&T Inc. Co. $\quad{ }^{2}$ - Marca registrada da Microsoft Inc. Co. } \\
\hline \multicolumn{4}{|c|}{$\begin{array}{l}3 \text { - Também modelo de reprodutor com pequenas modificaçóes } \\
5 \text { - Maximização de Esperanças } \\
6 \text { - Apenas efeitos aleatórios não correlacionados com animal }\end{array}$} \\
\hline $\begin{array}{l}\text { 5 - Maximização de Esperançac } \\
\text { - Adaptadas pelo autor. Don }\end{array}$ & público & nas efeitos aleatórios & o correlacionados com animal \\
\hline
\end{tabular}

\subsubsection{Critérios de Convergência}

- Sistemas DFREML (programas DFUNI, DFMUV e DFMUW) e MTDFREML (programa MTDFRUN)

- Método Simplex

O processo encerra-se quando o programa atinge o número máximo de iterações 
informado, ou quando a variancia do simplex for menor do que um valor especificado, pelo usuário. $\mathrm{O}$ valor padrão adotado no sistema DFREML é $\operatorname{Var}\left(-2 \log \left(L_{2}\right)\right)<10^{-8}$ e no sistema MTDFREML é $\operatorname{Var}\left(-2 \log \left(L_{2}\right)\right)<10^{-9}$.

\section{- Método de Powell}

Opção disponfvel apenas para os programas do sistema DFREML.

O processo se encerra quando o programa atinge o número máximo de iterações especificado, ou quando a logarítmo da função de verossimilhança restrita alterar, entre duas iterações subsequientes, menos do que um valor especificado pelo usuário (o valor padrão adotado pelo sistema é $\mathbf{0 . 0 0 0 1}$ ), ou quando o valor dos parâmetros a serem estimados alterarem um percentual menor do que o especificado pelo usuário (o valor padrão adotado pelo sistema é $2 \%$ ) entre iterações subsequientes.

OBS: - O processo de estimação deve ser repetido (tanto utilizando-se o método simplex quanto o método de Powell), até que a diferença de valores em $-2 \log \left(\mathrm{L}_{2}\right)$, entre duas "rodadas" completas, seja inferior a 0.002 unidade.

\section{- Sistema MTC}

Para o programa MTC, deve-se considerar a convergência alcançada quando a variável ConvR, a soma de quadrados dos desvios dos componentes de covariancia para o erro e ConvG, a soma de quadrados dos desvios dos componentes de covariancias entre classes de fatores aleatórios, obtidos entre iterações subsequientes, forem menores do que $10^{-6}$. Além disso, recomenda-se que a soma dos elementos fora da diagonal da matriz $G$ seja inferior a 0.01 . Neste último caso, para modelos com mais de um fator aleatório, além do erro, deve-se adotar o valor especificado com maior flexibilidade, pois neste caso normalmente não se consegue uma diagonalização perfeita das matrizes $G$ e $P$, que são diagonalizadas de uma forma conjunta.

\subsection{Dados Utilizados no Estudo}

\subsubsection{Dados Simulados}

Para se fazer um estudo comparativo entre os sistemas DFREML, MTC e MTDFREML, tendo em vista a estimação de componentes de covariancia, são utilizados diversos conjuntos de dados simulados, cada um com algumas características particulares, e um conjunto de dados de campo.

O primeiro conjunto de dados (arquivo UNIVA282.ARQ) foi extraído do trabalho de MEYER (1989). São dados simulados de 282 animais em duas gerações. Cada geração consiste de 18 famflias de irmãos completos de tamanho 6 a 10, e cada macho é acasalado com três fêmeas. Os pais das gerações não têm registro de produção, consistindo-se de 24 animais, perfazendo um total de 306 animais no total. O modelo animal adotado para análise comparativa é o 2 (Tabela 7), especificado por MEYER (1989), que inclui os efeitos fixos (três efeitos com 12 classes no total) e os efeitos aleatórios 
devido a animal (306 classes), efeito permanente de meio (efeito não correlacionado com animal, devido a registros repetidos de uma mesma mãe) (com 36 classes) e efeito do erro. $\mathrm{O}$ presente conjunto de dados simulados corresponde à medida de uma única característica, consistindo-se de uma análise univariada.

O segundo conjunto de dados é o arquivo D221 simulado por VALENTE (1988), correspondente a 107 registros de 10 reprodutores com 10 classes de efeitos fixos e medida de 2(duas) características. Essa análise é realizada para verificar a possibilidade de se processar, nos três sistemas, um modelo de reprodutor, no qual não se dispõe de informação de parentesco entre os animais.

O terceiro conjunto de dados analisados corresponde às informações do arquivo D411R2, simuladas por VALENTE (1988, pág. 26). Trata-se de uma análise multivariada com 3000 registros para cada uma das quatro caracteristicas. Esses dados são utilizados para estudar a estimação de componentes de covariancia para um modelo de reprodutor (com 30 classes) sem informação de parentesco entre individuos $(A=I)$. 0 modelo 1 (Tabelas $7 \mathrm{a}$ e $7 \mathrm{~b}$ ) estudado inclui os efeitos de animal (reprodutor) e de 20 classes de rebanho-ano-época (HYS).

Os dados dos arquivos D221 e D411R2 foram analisados somente pelos sistemas MTC e MTDFREML. Não se conseguiu fazê-lo pelo sistema DFREML, pois este não permite a análise de modelos nos quais todos pais e mães dos animais são desconhecidos. As análises desses dois arquivos também têm por objetivo verificar a performance dos sistemas no processo de estimação de componentes de (co)variancia para dados nos quais as MME's são pouco ou não esparsas.

O quarto conjunto de dados (MUEX2M.D) foi extrardo de um artigo publicado por MEYER (1991). Trata-se de um experimento de seleção em camundongos. Foram medidos o peso às seis semanas de idade e a ingestão de alimentos de quatro a seis semanas (ajustado pelo peso dentro de famflias às 4 semanas e por sexo) para três gerações de camundongos, selecionados pela quantidade de alimento ingerido. Os dados sâo de um total de 339 animais, sendo 284 com e 55 sem registros. Esses dados são analisados por um modelo linear multivariado (modelo animal), com os efeitos fixos de geração, sexo e tamanho de ninhada, respectivamente com três, duas e sete classes por caracteristica, e os efeitos aleatórios de animal (339 classes), efeito de ninhada como efeito aleatório adicional não correlacionado (42 classes) e efeito de erro.

Esse conjunto de dados foi analisado pelos quatro programas DFMUV, DFMUW, MTC e MTDFREML.

Adotam-se diferentes vetores de valores iniciais para estudar o efeito desses nas estimativas finais e na performance geral dos sistemas.

Em primeiro lugar, os valores iniciais utilizados são próximos aos componentes estimados por MEYER (1991). Em seguida, adotam-se vetores de valores iniciais iguais, 10 ou 0.1 vezes ao vetor de valores iniciais sugeridos por MEYER (1991). Posteriormente, variam-se os valores preliminares para cada uma das matrizes $G, P$ ou $R$. Essas matrizes representam as covariancias genética aditiva, devido a efeito permanente de meio e do erro, respectivamente. Neste caso, tomando-se por base os valores iniciais sugeridos por MEYER (1991), adotam-se, para uma das matrizes, valores 10 e 0.1 vezes àqueles, fixando-se os demais componentes das outras duas matrizes. Este processo é adotado para 
todas as três matrizes $G, P$ ou $R$. Finalmente, quando adotam-se os procedimentos livres de derivadas (DF), procede-se à análise utilizando-se uma estratégia especial, denominada estratégia $\mathrm{B}$, que consiste em se estimar os componentes em diversos passos, podendo ser assim descrita:

\section{- ESTRATÉGIA B}

- Passo 1 : Análises univariadas para cada uma das caracterı́sticas;

- Passo 2 : Análise multivariada, fixando-se as variancias aos valores encontrados na análise univariada, estimando-se as covariancias com baixa precisão ( $\operatorname{Var}\left(-2 \log \left(\mathrm{L}_{2}\right)\right)$ $\left.<10^{-3}\right)$;

- Passo 3 : Análise multivariada, com os valores preliminares obtidos no passo anterior, sem se fixar qualquer componente e adotando-se baixa precisão $\left(\operatorname{Var}\left(-2 \log \left(\mathrm{L}_{2}\right)\right)\right.$ $\left.<10^{-3}\right)$

- Passo 4 : Repetição do passo anterior para alto nfvel de precisão, isso é, Var($\left.2 \log \left(\mathrm{L}_{2}\right)\right)<10^{-9}$

- Passo 5 : Repetição do passo anterior, até que a diferença em -2Log $\left(\mathrm{L}_{2}\right)$, entre repeticões sucessivas, seja menor do que 0.002 unidade;

Essa estratégia é similar à sugerida por BOLDMAN et al. (1993, pág 52). Ela é possivel de ser executada somente para os programas dos sistemas DFREML e MTDFREML, ou seja sistemas que adotam o método livre de derivadas.

Outras estratégias de análises são adotadas, utilizando-se os métodos livres de derivadas. Estas, consistem na adoção de parte da estratégia B, especialmente o passo 1, para se estimar as variancias e utilizá-las como valores iniciais no processo de estimação, em análise multivariada. Outra estratégia é a execução de análise uni e bivariada, obtendo-se as estimativas de variancias e covariancias, para serem utilizadas como valores preliminares na análise multivariada final (com 3 características)

Além das análises dos conjuntos de dados cedidos pelos autores de diversas literaturas citadas, simulam-se novos conjuntos de dados, a partir de critérios especificos, procurando-se analisar o comportamento e adequação dos diversos sistemas, sobre uma variedade de condições. Para isso, são realizadas:

- Simulação de dados admitindo-se um modelo animal que inclui os efeitos fixos, e efeito de animal como única fonte de variação aleatória, além do erro. Para este caso são simulados dados baseando-se em 4 (quatro) diferentes matrizes de covariancia, para os efeitos genéticos aditivos $(G)$ e efeito do residuo $(R)$ (Tabela 6). Essas matrizes apresentam diferentes graus de condicionamento;

- Simulação de dados admitindo-se um modelo animal que inclui os efeitos fixos (HYS) e os aleatórios de animal e do erro. Além disso, é incluido um efeito aleatório adicional não correlacionado com animal, devido a registros repetidos de uma mesma 
vaca (modelo de repetibilidade). Para este caso, os dados são simulados baseando-se em três diferentes matrizes de covariância: dos efeitos genéticos aditivos $(G)$, do efeito permanente de meio (registros repetidos) $(P)$ e efeito do residuo $(R)$ (Tabela 6a.);

- Em todos casos são simulados valores para $\mathrm{q}=3$ características, adotando-se a decomposição de Cholesky das matrizes de parentesco $(A)$ e das matrizes $G$ (efeitos genéticos aditivos), $P$ (efeitos permanentes de meio) e $R$ (efeito do erro) (veja (2.4), pág. 58), de acordo com o modelo adotado;

- Para alguns arquivos de dados simulados, provoca-se perda aleatória simulada e independente de dado, para as características 2 ou 3. Este procedimento é realizado para avaliar possiveis alterações nos valores estimados dos componentes de covariância, decorrentes de mudanças na estrutura de correlações entre as características estudadas;

- No processo de simulação de variáveis, são utilizadas rotinas especificas para geração de variáveis com distribuição normal ${ }^{5}$, utilizando-se o procedimento descrito por KINDERMANN \& RAMAGE (1976), em que a distribuição de cada variável é aproximada por uma mistura de distribuições, denominadas região caudal e região central;

Em primeiro lugar são simulados quatro conjuntos de dados (arquivos MULTI282.SEP, MULTI615.SEP, MULTI615.ARQ e MULTI615.MIS), a partir da estrutura do arquivo apresentado por MEYER (1989), que serviu apenas para cálculo da matriz de parentesco $(A)$ entre animais.

Os dois primeiros arquivos de dados são gerados pela simulação de um modelo animal incluindo-se os efeitos fixos de rebanho-ano-época (HYS), e efeito aleatório de animal (306 classes) além do erro. Para o primeiro arquivo, a matriz utilizada no processo de simulação é a de número 4 e no segundo a de número 2 , apresentadas na tabela 6 .

$\mathrm{O}$ terceiro arquivo de dados é gerado pela simulação de um modelo animal que inclui os efeitos fixos de HYS e OP e os efeitos aleatórios de animal (matriz $G$ ), efeito permanente de meio (matriz $P$ ) devido a registros repetidos de uma mesma vaca e efeito do erro (matriz $R$ ). Para este arquivo, adota-se a matriz número 2 da tabela 6 a. Finalmente, o arquivo MULTI615. MIS é gerado pela perda de dados do arquivo MULTI615.ARQ.

Os demais conjuntos de dados são simulados com base em procedimento próprio, consistindo-se de três passos:

1. Geração de amostras de tamanho N, em programa especifico, baseando-se em famf́lias de meio-irmãs e irmãs completas;

\footnotetext{
${ }^{5}$ Rotinas de dominio público escritas por MEYER (1988)
} 
2. Cálculo da matriz de parentesco de animais (com base na amostra gerada no item anterior) utilizando-se um programa escrito por VALENTE $(1988)^{6}$ segundo o procedimento de QUAAS (1976) que considera endogamia;

3. Simulação de valores para $\mathrm{q}(\mathrm{q}=3)$ características, baseando-se em matrizes de componentes de covariancias para os diferentes fatores do modelo.

- São simulados modelos animal que incluem os efeitos fixos de HYS e os efeitos aleatórios representados pelas matrizes $G$ e $R$, para o caso em que cada animal tem uma única medida. Por outro lado, para modelos animal em que se disponha de registros repetidos por animal, o modelo inclui os efeitos fixos de HYS e ordem de parição (OP), e os efeitos dos fatores aleatórios representados pelas matrizes $G, P$ e $R$.

- Simulam-se três características para situação de igualdade de matriz de delineamento (sem valores perdidos) e situações de perda de dados para uma ou mais características (diferentes matrizes de delineamento por característica).

- Para alguns arquivos de dados simulados, provoca-se perda aleatória simulada e independente de $20 \%$ das observações para as características 2 e 3 . Este procedimento é realizado para avaliar possiveis alterações nos valores estimados dos componentes de covariância, decorrentes de mudanças na estrutura de correlação entre as características e para comparar os sistemas DFREML, MTDFREML com o MTC que em casos de perda de algum dados implica na perda total do registro.

- Um dos arquivos de dados simulados (SIMUL050.BEM) é analisado sob quatro condições:

a) - Análise de dados balanceados (arquivo SIMUL050.BEM), sem perda de qualquer registro. Utilizam-se os sistemas DFREML, MTC e MTDFREML;

b) - Perda simulada de $20 \%$ das observações para as características 2 ou 3 ;

b-1) Análise considerando-se os dados desbalanceados (arquivo SIMUL050.MIS). Utilizam-se os sistemas DFREML e MTDFREML. O sistema MTC não faz análise quando há esse tipo de desbalanceamento;

b-2) - Análise de dados balanceados (arquivo SIMUL050.PER). Quando há perda de dado, todo registro é considerado perdido. Os três sistemas são utilizados;

c) - Perda de todo(s) registro(s) posterior(es), quando no primeiro registro do animal o valor da característica 1 apresenta valor menor do que 3700 unidades.

c-1) Análise de dados balanceados (arquivo SIMUL050.SEL). Utilizam-se os três sistemas.

- Na tabela 6b. apresentam-se os valores dos parâmetros genéticos $\left(h^{2}, r\right.$ e $r_{g}$ ) e fenotípicos $\left(r_{p}\right)$, calculados com base nos valores dos componentes de covariancias adotados para simulação e mostrados nas tabelas 6 e 6 a.

\footnotetext{
${ }^{6}$ Programa de dominio público
} 
A tabela 6c. mostra uma descrição dos arquivos cujos dados são analisados. Todos arquivos com a terminação. MIS correspondem à situação de perda de dados a partir do arquivo original. Arquivos cujos dados são gerados considerando-se não haver registro repetido para os animais apresentam a terminação .SEP. Neste caso, o efeito permanente de meio não é considerado. Demais situações serão comentadas na discussão dos resultados.

Tabela 6. Matrizes de covariâncias utilizadas para simulação dos dados para modelo animal sem efeito permanente de meio

\begin{tabular}{|c|c|c|c|c|c|}
\hline \multirow{2}{*}{$\begin{array}{l}\text { Ma- } \\
\text { triz }\end{array}$} & \multirow[t]{2}{*}{ Parámetro } & \multicolumn{4}{|c|}{ Número da Matriz } \\
\hline & & 1 & 2 & 3 & 4 \\
\hline \multirow{7}{*}{ G } & $\overline{\sigma_{a_{1}}^{2}}$ & 173000,00 & 173000,00 & 160000,00 & 180000,00 \\
\hline & $\sigma_{a_{12}}$ & 800,00 & 2577,44 & 5959,22 & 3500,00 \\
\hline & $\sigma_{a_{13}}$ & 2200,00 & 2325,13 & 592,65 & 1800,00 \\
\hline & $\sigma_{a_{2}}^{2}$ & 300,00 & 240,00 & 307,20 & 230,00 \\
\hline & $\sigma_{a_{23}}$ & $-30,00$ & $-51,96$ & 159,81 & 20,00 \\
\hline & $\sigma_{a_{3}}^{2}$ & 190,00 & 125,00 & 219,52 & 180,00 \\
\hline & № Condição & 1146,71 & 3791,99 & 75206,12 & 1225,84 \\
\hline \multirow{7}{*}{$\mathbf{R}$} & $\overline{\sigma_{e_{1}}^{2}}$ & $\overline{415000,00}$ & 385000,00 & 352000,00 & 385000,00 \\
\hline & $\sigma_{e_{12}}$ & $-3700,00$ & $-4195,50$ & 12350,77 & $-4000,00$ \\
\hline & $\sigma_{e_{13}}$ & $-3500,00$ & $-4522,30$ & $-2994,82$ & $-3500,00$ \\
\hline & $\sigma_{e_{2}}^{2}$ & 508,00 & 508,00 & 512,00 & 508,00 \\
\hline & $\sigma_{e_{23}}$ & 246,00 & 246,41 & 45,69 & 200,00 \\
\hline & $\sigma_{e_{3}}^{2}$ & 332,00 & 332,00 & 407,68 & 332,00 \\
\hline & № Condição & 2639,07 & 2514,29 & 21403,98 & 1927,59 \\
\hline \multicolumn{6}{|c|}{ Outras informações } \\
\hline \multicolumn{2}{|c|}{ Característica } & & & & \\
\hline 1 & & 4000,00 & 4000,00 & 4000,00 & 4000,00 \\
\hline 2 & Média & 160,00 & 160,00 & 160,00 & 160,00 \\
\hline 3 & & 140,00 & 140,00 & 140,00 & 140,00 \\
\hline 1 & & 19,17 & 18,67 & 17,89 & 18,79 \\
\hline 2 & CV (\%) & 17,17 & 17,09 & 17,89 & 16,98 \\
\hline 3 & & 16,32 & 15,27 & 17,89 & 16,16 \\
\hline
\end{tabular}


Tabela 6a. Matrizes de covariancias utilizadas para simulação de modelos animal com efeito permanente de meio

\begin{tabular}{|c|c|c|c|c|}
\hline \multirow{2}{*}{$\begin{array}{l}\text { Ma- } \\
\text { triz }\end{array}$} & \multirow[t]{2}{*}{ Parâmetro } & \multicolumn{3}{|c|}{ Número da Matriz } \\
\hline & & 1 & $\begin{array}{r}2 \\
\end{array}$ & 3 \\
\hline \multirow{7}{*}{ G } & $\sigma_{a_{1}}^{2}$ & 173000,00 & 173000,00 & 160000,00 \\
\hline & $\sigma_{a_{12}}$ & 2880,00 & 2577,44 & 5959,22 \\
\hline & $\sigma_{a_{13}}$ & 3690,00 & 2325,13 & 592,65 \\
\hline & $\sigma_{a_{2}}^{2}$ & 240,00 & 240,00 & 307,20 \\
\hline & $\sigma_{a_{23}}$ & 104,00 & $-51,96$ & 159,81 \\
\hline & $\sigma_{a_{3}}^{2}$ & 125,00 & 125,00 & 219,52 \\
\hline & No Condição & 4980,26 & 3791,99 & 75206,12 \\
\hline \multirow{7}{*}{$\mathbf{P}$} & $\sigma_{p_{1}}^{2}$ & 165000,00 & 165000,00 & 128000,00 \\
\hline & $\sigma_{p_{12}}$ & 4500,00 & 1795,12 & 4730,00 \\
\hline & $\sigma_{p_{13}}$ & 4410,00 & 968,09 & $-2077,83$ \\
\hline & $\sigma_{p_{2}}^{2}$ & 217,00 & 217,00 & 204,80 \\
\hline & $\sigma_{p_{23}}$ & 145,00 & $-17,55$ & $-22,30$ \\
\hline & $\sigma_{p_{3}}^{2}$ & 142,00 & 142,00 & 156,80 \\
\hline & No Condição & 10145,55 & 1316,31 & 26247,30 \\
\hline \multirow{7}{*}{$\mathbf{R}$} & $\overline{\sigma_{e_{1}}^{2}}$ & 385000,00 & 385000,00 & 352000,00 \\
\hline & $\sigma_{e_{12}}$ & 10500,00 & $-4195,50$ & 12350,77 \\
\hline & $\sigma_{e_{13}}$ & 10290,00 & $-4522,30$ & $-2994,82$ \\
\hline & $\sigma_{e_{2}}^{2}$ & 508,00 & 508,00 & 512,00 \\
\hline & $\sigma_{e_{23}}$ & 339,00 & 246,41 & 45,69 \\
\hline & $\sigma_{e_{3}}^{2}$ & 332,00 & 332,00 & 407,68 \\
\hline & № Condição & 10046,81 & 2514,29 & 21403,98 \\
\hline \multicolumn{5}{|c|}{ Outras informações } \\
\hline \multicolumn{2}{|c|}{ Característica } & & & \\
\hline 1 & \multirow{3}{*}{ Média (kg) } & 4000,00 & 4000,00 & 4000,00 \\
\hline 2 & & 160,00 & 160,00 & 160,00 \\
\hline 3 & & 140,00 & 140,00 & 140,00 \\
\hline 1 & \multirow{3}{*}{$\mathrm{CV}(\%)$} & 21,26 & 21,26 & 20,00 \\
\hline 2 & & 19,42 & 19,42 & 20,00 \\
\hline 3 & & 17,48 & 17,48 & 20,00 \\
\hline
\end{tabular}


Tabela 6 b. Herdabilidade $\left(h^{2}\right)$, repetibilidade $(r)$ e correlações genéticas $\left(r_{g}\right)$ e fenotípicas $\left(r_{p}\right)$, para os dados das tabelas 6 e $6 a$

\begin{tabular}{|l|rrrr|rrr|}
\hline \multirow{2}{*}{ Parâ- } & \multicolumn{4}{|c|}{ Tabela 6 } & \multicolumn{3}{c|}{ Tabela 6a } \\
\cline { 2 - 8 } & \multicolumn{3}{|c|}{ Matriz } & \multicolumn{3}{c|}{ Matriz } \\
\cline { 2 - 8 } & $(1)$ & $(2)$ & $(3)$ & $(4)$ & $(1)$ & $(2)$ & $(3)$ \\
\hline$h_{1}^{2}$ & 0,29 & 0,31 & 0,31 & 0,32 & 0,24 & 0,24 & 0,25 \\
$h_{2}^{2}$ & 0,37 & 0,32 & 0,37 & 0,31 & 0,25 & 0,25 & 0,30 \\
$h_{3}^{2}$ & 0,36 & 0,27 & 0,35 & 0,35 & 0,21 & 0,21 & 0,28 \\
\hline$r_{1}$ & - & - & - & - & 0,47 & 0,47 & 0,45 \\
$r_{2}$ & - & - & - & - & 0,47 & 0,47 & 0,50 \\
$r_{3}$ & - & - & - & - & 0,45 & 0,45 & 0,48 \\
\hline$r_{g_{12}}$ & 0,11 & 0,40 & 0,85 & 0,54 & 0,45 & 0,40 & 0,85 \\
$r_{g_{13}}$ & 0,38 & 0,50 & 0,10 & 0,32 & 0,79 & 0,50 & 0,10 \\
$r_{g_{23}}$ & $-0,13$ & $-0,30$ & 0,61 & 0,10 & 0,60 & $-0,30$ & 0,61 \\
\hline$r_{p_{12}}$ & $-0,13$ & $-0,08$ & 0,89 & $-0,02$ & 0,68 & 0,00 & 0,90 \\
$r_{p_{13}}$ & $-0,07$ & $-0,14$ & $-0,13$ & $-0,10$ & 0,88 & $-0,06$ & $-0,20$ \\
$r_{p_{23}}$ & 0,33 & 0,33 & 0,29 & 0,35 & 0,73 & 0,23 & 0,20 \\
\hline
\end{tabular}

\subsubsection{Dados Reais}

Além dos dados simulados, realizam-se análises de um conjunto de dados de campo (CNPGL001.ARQ). Neste caso, utilizam-se os dados de produção de leite de rebanhos da raça Gir leiteiro, extrafdos do arquivo zootécnico nacional, mantidos na EMBRAPA/CNPGL. Os dados foram previamente verificados, recodificando-se apropriadamente a numeração de animais (vaca, pai e mãe). Além disso, usando-se critérios adotados na EMBRAPA/CNPGL, todos registros cujo encerramento da lactação é considerado anormal foram eliminados. Também foram eliminados registros cujo pai da vaca tinha menos do que duas filhas, dados referentes a ano de parto com menos de 30 observaçôes e ordem de parto (OP) maior do que 3. Foram selecionadas apenas as vacas da raça Gir PO (puro de origem) ou PC (puro por cruza).

Ao final da consistência e eliminação de informações, resultaram um total de 3699 lactações, correspondentes a 1718 vacas, filhas de 142 reprodutores e de 488 mães, das quais 144 também produziram leite. Os efeitos fixos são representados por 301 classes de rebanho-ano-época (HYS), duas classes de composição genética (CG) (1 - Puro de Origem e 2 - Puro por cruza) e três classes de ordem de partos (OP).

Esses dados são analisados por análise univariada, como um modelo de reprodutor (desprezando-se toda informação de parentesco entre os animais) e como um modelo animal, considerando-se os efeitos fixos de HYS, CG e OP, e os efeitos aleatórios de animal, efeito permanente de meio e efeito do erro.

Posteriormente, consideram-se as produções nas lactações 1,2 e 3, como três diferentes caracterfsticas e procede-se à análise uni e multivariada, admitindo-se um 
modelo animal que inclui os efeitos fixos de HYS e CG e os efeitos aleatórios de animal e do erro. Para a análise considerando-se o modelo de reprodutor, são excluidos os registros de produção da vaca cujo pai apresenta numeração 0 (desconhecido). O número de registros para este caso foi reduzido para 3307.

Tabela 6c. Descrição dos arquivos de dados utilizados nas análises

\begin{tabular}{|c|c|c|c|c|c|c|c|c|c|c|}
\hline \multirow{2}{*}{$\begin{array}{l}\text { Nome do } \\
\text { Arquivo }\end{array}$} & \multirow{2}{*}{$\begin{array}{l}\text { Balan- } \\
\text { ceado }\end{array}$} & \multirow[b]{2}{*}{$(1)$} & \multirow{2}{*}{$\begin{array}{l}\text { No. } \\
\text { Reg. }\end{array}$} & \multirow[b]{2}{*}{$(2)$} & \multirow[b]{2}{*}{$(3)$} & \multirow[b]{2}{*}{$(4)$} & \multirow[b]{2}{*}{$(5)$} & \multicolumn{3}{|c|}{ No. de Registros } \\
\hline & & & & & & & & $y_{1}$ & $y_{2}$ & $y_{3}$ \\
\hline \multicolumn{11}{|c|}{ Dados Extraidos da Literatura } \\
\hline UNIVA282.ARQ & Sim & 1 & 282 & 306 & 24 & 2 & 12 & 282 & - & - \\
\hline MUEX2M.D & Sim & 2 & 282 & 306 & 24 & 2 & 24 & 282 & 282 & - \\
\hline D221 & Sim & 2 & 107 & 10 & 10 & 2 & 20 & 107 & 107 & - \\
\hline $\mathrm{D} 411 \mathrm{R} 2$ & Sim & 4 & 3000 & 30 & 30 & 2 & 80 & 3000 & 3000 & 3000 \\
\hline \multicolumn{11}{|c|}{ Dados Simulados } \\
\hline MULTI615.ARQ & Sim & 3 & 615 & 306 & 24 & 3 & 117 & 615 & 615 & 615 \\
\hline MULTI615.MIS & Não & 3 & 615 & 306 & 24 & 3 & 117 & 615 & 509 & 488 \\
\hline MULTI615.SEP & Sim & 3 & 282 & 306 & 24 & 3 & 108 & 282 & 282 & 282 \\
\hline MULTI282.SEP & Sim & 3 & 282 & 306 & 24 & 2 & 2 & 282 & 282 & 282 \\
\hline SIMUL050.BEM & Sim & 3 & 301 & 185 & 35 & 2 & 132 & 301 & 301 & 301 \\
\hline SIMUL050.MIS* & Não & 3 & 301 & 185 & 35 & 2 & 132 & 301 & 261 & 204 \\
\hline SIMUL050.PER & Sim & 3 & 204 & 185 & 35 & 2 & 132 & 204 & 204 & 204 \\
\hline SIMUL050.SEL & Sim & 3 & 238 & 185 & 35 & 2 & 132 & 238 & 238 & 238 \\
\hline SIMUL050.MAL & Sim & 3 & 301 & 185 & 35 & 2 & 132 & 301 & 301 & 301 \\
\hline SIMUL100.SEP & Sim & 3 & 400 & 500 & 100 & 2 & 186 & 400 & 400 & 400 \\
\hline SIMUL100.ARQ & Sim & 3 & 914 & 500 & 100 & 3 & 249 & 914 & 914 & 914 \\
\hline SIMUL100.PER & Não & 3 & 914 & 500 & 100 & 3 & 186 & 914 & 861 & 879 \\
\hline SIMUL400.ARQ & Sim & 3 & 3713 & 1800 & 200 & 3 & 369 & 3713 & 3713 & 3713 \\
\hline SIMUL400.MIS & Não & 3 & 3713 & 1800 & 200 & 3 & 360 & 3713 & 2957 & 2984 \\
\hline SIMUL400.SEP & Sim & 3 & 1600 & 1800 & 200 & 2 & 354 & 1600 & 1600 & 1600 \\
\hline SIMUL400.PER & Não & 3 & 1600 & 1800 & 200 & 3 & 353 & 1600 & 1111 & 1142 \\
\hline \multicolumn{11}{|c|}{ Dados Reais } \\
\hline CNPGL001.ARQ ${ }^{*}$ & Não & 3 & 3699 & 2365 & 647 & 2 & 797 & 1718 & 1142 & 839 \\
\hline $\begin{array}{l}(1)=\text { № de caracteris } \\
(4)=\text { № de Efeitos A }\end{array}$ & $\begin{array}{l}\text { icas } \\
\text { eatórios }\end{array}$ & $\begin{array}{l}(2)= \\
(5)=\end{array}$ & $\begin{array}{l}=N^{\circ} \text { tot } \\
=N^{\circ} \mathrm{Cla}\end{array}$ & Efeito & Fixos & & $\begin{array}{l}(3)=\mathrm{N} \\
=\text { And }\end{array}$ & $\begin{array}{l}\text { Anima } \\
\text { lises Un }\end{array}$ & $\begin{array}{l}\text { is Sem F } \\
\text { e Multi }\end{array}$ & $\begin{array}{l}\text { egistro } \\
\text { variada }\end{array}$ \\
\hline
\end{tabular}

\subsection{Modelo Linear Misto Multivariado}

Para o presente estudo, será adotado o modelo linear misto multivariado para $q$ características e $r$ fatores aleatórios, já considerado em ((4), pág. 27):

$$
y=X \beta+Z u+\varepsilon
$$

em que: 
- $y$ é um vetor de $\mathrm{N}$ observações para todas caracteristicas. $N=n_{1}+n_{2}+\ldots+n_{q}$. $n_{i}$ é o número de observações para a caracteristica i;

- $\beta$ é um vetor de NF classes de efeitos fixos (incluindo covariáveis com efeito linear ou ordens superiores);

- $X$ é a matriz de delineamento ou matriz de incidencia de dimensão $\mathrm{N}^{*} \mathrm{NF}$ para os efeitos fixos, com posto coluna $N F^{*}$;

- $u$ é um vetor de todas NR classes dos efeitos aleatórios, além do erro;

- $Z$ é a matriz de incidencia de dimensão $\mathrm{N}^{*} \mathrm{NR}$ para os efeitos aleatórios; e

- $\varepsilon$ é um vetor de dimensão $\mathrm{N}$ dos erros aleatórios.

Sejam:

$\mathrm{V}(u)=G$ a matriz de (co)variância entre classes dos fatores aleatórios;

$\mathrm{V}(\varepsilon)=R$, a matriz de (co)variancia entre os efeitos residuais; e

$\operatorname{Cov}\left(u, \varepsilon^{\prime}\right)=\emptyset$ a matriz de covariâncias entre fatores aleatórios e efeitos residuais.

Além disso, sejam:

$\mathrm{E}(u)=\mathrm{E}(\varepsilon)=\emptyset, \quad \mathrm{E}(y)=X \beta \quad$ e $\mathrm{V}(y)=V=Z G Z^{\prime}+R$.

Assim, admitindo-se normalidade, tem-se:

$$
y=X \beta+Z u+\varepsilon \stackrel{i i d}{\sim} N(X \beta, V) .
$$

cuja função densidade de probabilidade de $y$ é dada em (8), (pág. 41)

As tabelas 7,7a. e 7b. apresentam os tipos de análises possivel de serem realizadas utilizando-se o modelo adotado e os sistemas DFREML, MTC e MTDFREML.

Neste trabalho, os dados são analisados sob os modelos tipo 1 ou 2, isso é, modelos que incluem um ou dois fatores aleatórios além do erro. Além disso, o efeito aleatório adicional, quando presente, é considerado não correlacionado com os demais efeitos aleatórios do modelo. 
Tabela 7. Modelos de análise para o sistema DFREML

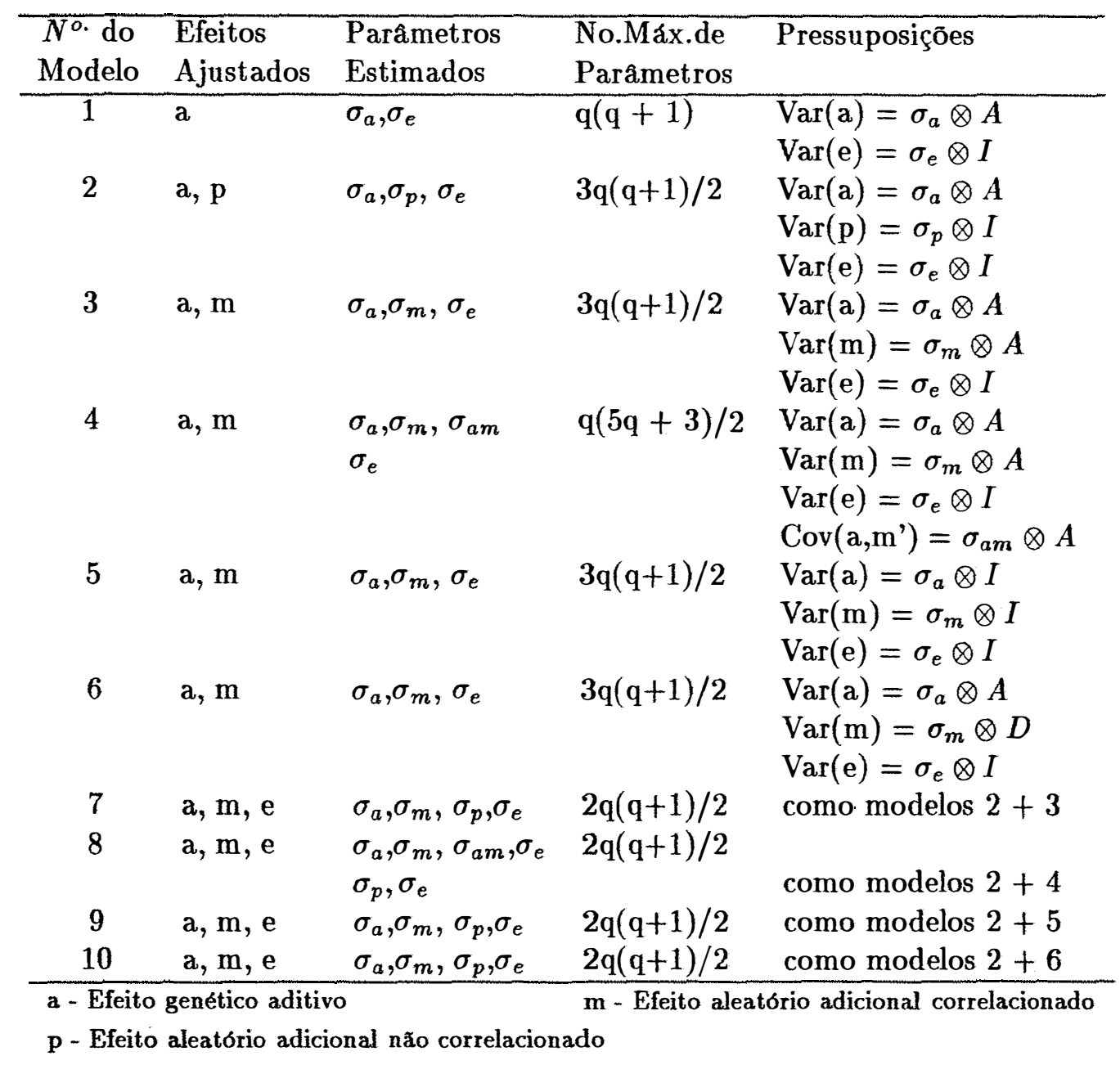

Tabela 7a. Modelos de análise para o sistema MTC

\begin{tabular}{|c|c|c|c|c|}
\hline $\begin{array}{l}N^{\circ} \text { do } \\
\text { Modelo }\end{array}$ & $\begin{array}{l}\text { Efeitos } \\
\text { Ajustados }\end{array}$ & $\begin{array}{l}\text { Parametros } \\
\text { Estimados }\end{array}$ & $\begin{array}{l}\text { No.Máx.de } \\
\text { Parametros }\end{array}$ & Pressuposiçōes \\
\hline 1 & $\mathbf{a}$ & $\sigma_{a}, \sigma_{e}$ & $q(q+1)$ & $\begin{array}{l}\operatorname{Var}(\mathrm{a})=\sigma_{a} \otimes I \\
\operatorname{Var}(\mathrm{e})=\sigma_{e} \otimes I\end{array}$ \\
\hline 2 & $\mathbf{a}$ & $\sigma_{a}, \sigma_{e}$ & $q(q+1)$ & $\begin{array}{l}\operatorname{Var}(\mathrm{a})=\sigma_{a} \otimes A \\
\operatorname{Var}(\mathrm{e})=\sigma_{e} \otimes I\end{array}$ \\
\hline 3 & $\mathrm{a}, \mathrm{p}$ & $\sigma_{a}, \sigma_{p}, \sigma_{e}$ & $3 q(q+1) / 2$ & $\begin{array}{l}\operatorname{Var}(\mathrm{a})=\sigma_{a} \otimes I \\
\operatorname{Var}(\mathrm{p})=\sigma_{p} \otimes I \\
\operatorname{Var}(\mathrm{e})=\sigma_{e} \otimes I\end{array}$ \\
\hline 4 & $\mathrm{a}, \mathrm{p}$ & $\sigma_{a}, \sigma_{p}, \sigma_{e}$ & $3 q(q+1) / 2$ & $\begin{array}{l}\operatorname{Var}(\mathrm{a})=\sigma_{a} \otimes A \\
\operatorname{Var}(\mathrm{p})=\sigma_{p} \otimes I \\
\operatorname{Var}(\mathrm{e})=\sigma_{e} \otimes I\end{array}$ \\
\hline
\end{tabular}

a - Efeito genético aditivo p - Efeito aleatório adicional não correlacionado 
Tabela 7b. Modelos de análise para o sistema MTDFREML

\begin{tabular}{|c|c|c|c|c|}
\hline $\begin{array}{l}N^{o \cdot} \text { do } \\
\text { Modelo }\end{array}$ & $\begin{array}{l}\text { Efeitos } \\
\text { Ajustados }\end{array}$ & $\begin{array}{l}\text { Parámetros } \\
\text { Estimados }\end{array}$ & $\begin{array}{l}\text { No.Máx.de } \\
\text { Parâmetros }\end{array}$ & Pressuposições\# \\
\hline 1 & $\mathrm{a}$ & $\sigma_{a}, \sigma_{e}$ & $q(q+1)$ & $\begin{array}{l}\operatorname{Var}(\mathrm{a})=\sigma_{a} \otimes A \\
\operatorname{Var}(\mathrm{e})=\sigma_{e} \otimes I\end{array}$ \\
\hline 2 & $\mathrm{a}, \mathrm{p}$ & $\sigma_{a}, \sigma_{p}, \sigma_{e}$ & $3 q(q+1) / 2$ & $\begin{array}{l}\operatorname{Var}(\mathrm{a})=\sigma_{a} \otimes A \\
\operatorname{Var}(\mathrm{p})=\sigma_{p} \otimes I \\
\operatorname{Var}(\mathrm{e})=\sigma_{e} \otimes I\end{array}$ \\
\hline 3 & $\mathrm{a}, \mathrm{m}$ & $\sigma_{a}, \sigma_{m}, \sigma_{e}$ & $3 q(q+1) / 2$ & $\begin{array}{l}\operatorname{Var}(\mathrm{a})=\sigma_{a} \otimes A \\
\operatorname{Var}(\mathrm{m})=\sigma_{m} \otimes A \\
\operatorname{Var}(\mathrm{e})=\sigma_{e} \otimes I\end{array}$ \\
\hline 4 & $\mathrm{a}, \mathrm{m}$ & $\begin{array}{l}\sigma_{a}, \sigma_{m}, \sigma_{a m} \\
\sigma_{e}\end{array}$ & $q(5 q+3) / 2$ & $\begin{array}{l}\operatorname{Var}(\mathrm{a})=\sigma_{a} \otimes A \\
\operatorname{Var}(\mathrm{m})=\sigma_{m} \otimes A \\
\operatorname{Var}(\mathrm{e})=\sigma_{e} \otimes I \\
\operatorname{Cov}\left(\mathrm{a}, \mathrm{m}^{\prime}\right)=\sigma_{a m} \otimes A\end{array}$ \\
\hline 5 & $\mathrm{a}, \mathrm{m}$ & $\sigma_{a}, \sigma_{m}, \sigma_{e}$ & $3 q(q+1) / 2$ & $\begin{array}{l}\operatorname{Var}(\mathrm{a})=\sigma_{a} \otimes I \\
\operatorname{Var}(\mathrm{m})=\sigma_{m} \otimes I \\
\operatorname{Var}(\mathrm{e})=\sigma_{e} \otimes I\end{array}$ \\
\hline 6 & $\mathrm{a}, \mathrm{m}$ & $\sigma_{a}, \sigma_{m}, \sigma_{e}$ & $3 q(-1) / 2$ & $\begin{array}{l}\operatorname{Var}(\mathrm{a})=\sigma_{a} \otimes A \\
\operatorname{Var}(\mathrm{m})=\sigma_{m} \otimes D \\
\operatorname{Var}(\mathrm{e})=\sigma_{e} \otimes I\end{array}$ \\
\hline 7 & $\mathrm{a}, \mathrm{m}, \mathrm{e}$ & $\sigma_{a}, \sigma_{m}, \sigma_{p}, \sigma_{e}$ & $2 q(q+1) / 2$ & como modelos $2+3$ \\
\hline 8 & $\mathrm{a}, \mathrm{m}, \mathrm{e}$ & $\begin{array}{l}\sigma_{a}, \sigma_{m}, \sigma_{a m}, \sigma_{e} \\
\sigma_{p}, \sigma_{e}\end{array}$ & $2 q(q+1) / 2$ & como modelos $2+4$ \\
\hline 9 & $\mathrm{a}, \mathrm{m}, \mathrm{e}$ & $\sigma_{a}, \sigma_{m}, \sigma_{p}, \sigma_{e}$ & $2 q(q+1) / 2$ & como modelos $2+5$ \\
\hline 10 & $\mathrm{a}, \mathrm{m}, \mathrm{e}$ & $\sigma_{a}, \sigma_{m}, \sigma_{p}, \sigma_{e}$ & $2 q(q+1) / 2$ & como modelos $2+6$ \\
\hline $\begin{array}{l}\#-A \\
m-E f\end{array}$ & pode ser $g$ & $\begin{array}{l}\text { izada ou (I)denti } \\
\text { rrelacionado }\end{array}$ & $\begin{array}{l}\text { a - Efeito ge } \\
\text { p - Efeito al }\end{array}$ & $\begin{array}{l}\text { aditivo } \\
\text { o adicional não correlacior }\end{array}$ \\
\hline
\end{tabular}

\subsection{Derivação das equações do modelo misto (MME)}

Segundo o modelo especificado em (31), admitindo-se normalidade, a distribuição conjunta de $y$ e $u$ é dada por

$$
\begin{aligned}
f(y, u) & =g(y / u) \cdot h(u) \\
& =\frac{1}{(2 \pi)^{\frac{\mathbf{n q}}{2}}|\mathbf{R}|^{\frac{1}{2}}} e^{-\frac{1}{2}\left[(y-X \beta-Z u)^{\prime} R^{-1}(y-X \beta-Z u)\right]} \cdot \frac{1}{(2 \pi)^{\frac{\mathbf{n s}}{2}}|\mathbf{G}|^{\frac{1}{2}}} e^{-\frac{1}{2}\left[u^{\prime} G^{-1} u\right] .}
\end{aligned}
$$

Em que nq e ns são os postos das matrizes $R$ e $G$, respectivamente.

Observe que, para derivação dos efeitos de $u$ e $\beta$, os termos em negrito são constantes e serão denotados por $k$. Assim, o logaritmo de $f(y, u)$ pode ser escrito como:

$$
l=\log (f(y, u))=k-\frac{1}{2}(y-X \beta-Z u)^{\prime} R^{-1}(y-X \beta-Z u)-\frac{1}{2} u^{\prime} G^{-1} u .
$$


Diferenciando-se essa função em relação aos vetores de parâmetros desconhecidos $\beta$ e $u$, obtém-se:

$$
\begin{aligned}
\frac{\partial l}{\partial \beta} & =X^{\prime} R^{-1}(y-X \beta-Z u)=X^{\prime} R^{-1} y-X^{\prime} R^{-1} X \beta-X^{\prime} R^{-1} Z u \\
\frac{\partial l}{\partial u} & =Z^{\prime} R^{-1}(y-X \beta-Z u)+G^{-1} u .
\end{aligned}
$$

Igualando essas equações a zero, resulta:

$$
\begin{aligned}
& \frac{\partial l}{\partial \beta}=0 \Rightarrow X^{\prime} R^{-1} X \hat{\beta}+X^{\prime} R^{-1} Z \hat{u}=X^{\prime} R^{-1} y \\
& \frac{\partial l}{\partial u}=0 \Rightarrow Z^{\prime} R^{-1} X \hat{\beta}+\left(Z^{\prime} R^{-1} Z+G^{-1}\right) \hat{u}=Z^{\prime} R^{-1} y \\
& \Rightarrow\left[\begin{array}{ll}
X^{\prime} R^{-1} X & X^{\prime} R^{-1} Z \\
Z^{\prime} R^{-1} X & Z^{\prime} R^{-1} Z+G^{-1}
\end{array}\right]\left[\begin{array}{l}
\hat{\beta} \\
\hat{u}
\end{array}\right]=\left[\begin{array}{c}
X^{\prime} R^{-1} y \\
Z^{\prime} R^{-1} y
\end{array}\right]
\end{aligned}
$$

Essas equações, deduzidas inicialmente por HENDERSON (1973) e por SEARLE (1979), são denominadas equações do modelo misto (MME).

Absorvendo os efeitos fixos $(\hat{\beta})$ nas equações para os efeitos aleatórios $(\hat{u})$, tem-se:

$$
\begin{aligned}
\hat{\beta}=\left(X^{\prime} R^{-1} X\right)^{-}\left(X^{\prime} R^{-1} y-X^{\prime} R^{-1} Z \hat{u}\right) & \mathrm{e} \\
Z^{\prime} R^{-1} X\left(X^{\prime} R^{-1} X\right)^{-}\left(X^{\prime} R^{-1} y-X^{\prime} R^{-1} Z \hat{u}\right)+\left(Z^{\prime} R^{-1} Z+G^{-1}\right) \hat{u} & =Z^{\prime} R^{-1} y \\
Z^{\prime} R^{-1} X\left(X^{\prime} R^{-1} X\right)^{-} X^{\prime} R^{-1} y-Z^{\prime} R^{-1} X\left(X^{\prime} R^{-1} X\right)^{-} X^{\prime} R^{-1} Z \hat{u} & =Z^{\prime} R^{-1} y \\
\left\{\left(Z^{\prime} R^{-1} Z+G^{-1}\right)-Z^{\prime} R^{-1} X\left(X^{\prime} R^{-1} X\right)^{-} X^{\prime} R^{-1} Z\right\} \hat{u} & =\left\{Z^{\prime} R^{-1} y-\right. \\
\left.Z^{\prime} R^{-1} X\left(X^{\prime} R^{-1} X\right)^{-} X^{\prime} R^{-1} y\right\} & \\
\left\{Z^{\prime} R^{-1} Z-Z^{\prime} R^{-1} X\left(X^{\prime} R^{-1} X\right)^{-} X^{\prime} R^{-1} Z+G^{-1}\right\} \hat{u} & =\left\{Z^{\prime} R^{-1} y-\right. \\
\left.Z^{\prime} R^{-1} X\left(X^{\prime} R^{-1} X\right)^{-} X^{\prime} R^{-1} y\right\} & \\
\Rightarrow\left\{Z^{\prime}\left[R^{-1}-R^{-1} X\left(X^{\prime} R^{-1} X\right)^{-} X^{\prime} R^{-1}\right] Z+G^{-1}\right\} \hat{u} & = \\
\left\{Z^{\prime}\left[R^{-1}-R^{-1} X\left(X^{\prime} R^{-1} X\right)^{-} X^{\prime} R^{-1}\right] y\right\} &
\end{aligned}
$$

Finalmente, fazendo-se

$$
S=R^{-1}-R^{-1} X\left(X^{\prime} R^{-1} X\right)^{-} X^{\prime} R^{-1}
$$

o sistema de equações resultante será:

$$
\begin{aligned}
& \left(Z^{\prime} S Z+G^{-1}\right) \hat{u}=Z^{\prime} S y \\
\Rightarrow \hat{u}= & \left(Z^{\prime} S Z+G^{-1}\right)^{-} Z^{\prime} S y .
\end{aligned}
$$


Para facilitar as deduçōes que serão apresentadas posteriormente, admitemse as seguintes matrizes e/ou vetores adicionais, alguns dos quais já considerados anteriormente:

- a) L uma combinação linear das observações, denominada contraste de erros, tal que $L^{\prime} X=\emptyset$. Por exemplo, seja $L^{\prime}=\left(I-X\left(X^{\prime} V^{-1} X\right)^{-} X^{\prime}\right)$;

- b) E, com elementos $e_{i j}$ uma matriz simétrica q x q de residuos ou covariancias do erro entre características;

- c) $\mathrm{T}=\left[t_{i j}\right]$ de dimensão rq $\mathrm{x}$ rq representando uma matriz de covariâncias entre efeitos aleatórios, em que $\mathrm{r}$ representa o número de fatores aleatórios no modelo (além do erro) e q o número de características. Assume-se que existam $r^{*} \leq r(r-$ 1)/2 covariâncias entre os $r$ fatores aleatórios. O número total de parâmetros a ser estimados é então $s=q(q+1)(r+1) / 2+q^{2} r^{*}$;

- d) $\theta$, com elementos $\theta_{i}, \mathrm{i}=1, \ldots, \mathrm{s}$, um vetor de componentes de (co)variância a serem estimados, incluindo os componentes do erro ou efeito do residuo;

- e) $P=V^{-1}-V^{-1} X\left(X^{\prime} V^{-1} X\right)^{-} X^{\prime} V^{-1}$

$$
=V^{-1}-V^{-1} X^{*}\left(X^{* *} V^{-1} X^{*}\right)^{-1} X^{\prime *} V^{-1} \text {. }
$$

em que $X^{*}$, de ordem $N$ x $N F^{*}$, representa uma submatriz de $X$ de posto coluna completo (é a matriz $X$ após imposição de restrições linearmente independentes a.os parâmetros (restrições não estimáveis), referente aos efeitos fixos do modelo).

- f) $V=\sum_{i=1}^{s} \frac{\partial}{\partial} \frac{V}{\theta_{i}} \theta_{i}$

- g) Propriedades Importantes: (veja, por exemplo, SEARLE, 1979)

$$
\begin{aligned}
& \left.\mathrm{g}_{1}\right) \begin{aligned}
P V P & =(\overbrace{V^{-1}-V^{-1} X\left(X^{\prime} V^{-1} X\right)^{-} X^{\prime} V^{-1}}^{P}) \\
& \overbrace{V}^{V}(\overbrace{V^{-1}-V^{-1} X\left(X^{\prime} V^{-1} X\right)^{-} X^{\prime} V^{-1}}^{P}) \\
\left.\mathrm{g}_{2}\right) P y & =V^{-1}(y-X \hat{\beta})
\end{aligned} \\
& \left.\mathrm{g}_{3}\right) L\left(L^{\prime} V L\right)^{-1} L=P
\end{aligned}
$$

O logarítmo da função de verossimilhança de $L^{\prime} y$, que foi apresentado em (25) (pág. 48), é dado por:

$$
L_{2}=-\frac{1}{2}\left[(N-r(X)) \log (2 \pi)+\log |R|+\log |G|+\log |C|+y^{\prime} P y\right] .
$$

Uma forma alternativa apresentada por SEARLE (1979) é:

$$
\begin{aligned}
L_{2}= & -\frac{1}{2}\left((N-r(X)) \log (2 \pi)+\log |V|+\log \left|X^{*^{\prime}} V^{-1} X^{*}\right|\right. \\
& \left.-\frac{1}{2}(y-X \hat{\beta})^{\prime} V^{-1}(y-X \hat{\beta})\right)
\end{aligned}
$$

em que $X^{*}$ já foi definido em (e). 


\subsection{Estimadores dos Componentes de Covariancia}

\subsubsection{Algoritmo EM (Maximização de Esperanças)}

Veja os resultados de (27), (pág. 50) e (29), (pág. 50).

Detalhes da diferenciação de $L_{2}$ em relação aos elementos de $\theta_{i}$ podem ser encontrados em ANDREONI (1989). Veja também CALVIN (1993).

Diferenciando $L_{2}$ de (32) em relação aos elementos de $\theta_{i}$, tem-se:

$$
\begin{aligned}
\frac{\partial L_{2}}{\partial \theta_{i}}= & \left.-\frac{1}{2}\left[\operatorname{Tr}\left(V^{-1} \frac{\partial V}{\partial \theta_{i}}\right)+\operatorname{Tr}\left(X^{*^{\prime}} V^{-1} X^{*}\right)^{-1} X^{*} V^{-1} \frac{\partial V}{\partial \theta_{i}} V^{-1} X^{*}\right)\right] \\
& +\frac{1}{2}\left[(y-X \hat{\beta})^{\prime} V^{-1} \frac{\partial V}{\partial \theta_{i}} V^{-1}(y-X \hat{\beta})\right] .
\end{aligned}
$$

Igualando essas equações a zero tem-se:

$$
\begin{gathered}
\operatorname{Tr}\left(V^{-1} \frac{\partial V}{\partial \theta_{i}}\right)-\operatorname{Tr}\left[\left(X^{*^{\prime}} V^{-1} X^{*}\right)^{-1} X^{*} V^{-1} \frac{\partial V}{\partial \theta_{i}} V^{-1} X^{*}\right] \\
=(y-X \hat{\beta})^{\prime} V^{-1} \frac{\partial V}{\partial \theta_{i}} V^{-1}(y-X \hat{\beta}) \quad \mathrm{e} \\
\left.\operatorname{Tr}\left[V^{-1}-V^{-1} X^{*}\left(X^{*^{\prime}} V^{-1} X^{*}\right)^{-1} X^{*} V^{-1} \frac{\partial V}{\partial \theta_{i}}\right]=(y-X \hat{\beta})^{\prime} V^{-1} \frac{\partial V}{\partial \theta_{i}} V^{-1}(y-X \hat{\beta})\right) .
\end{gathered}
$$

Considerando-se (e) e $\left(g_{2}\right)$, as equações REML tornam-se:

$$
\operatorname{Tr}\left[P \frac{\partial V}{\partial \theta_{i}}\right]=y^{\prime} P \frac{\partial V}{\partial \theta_{i}} P y \text { para } \mathrm{i}=1, \ldots, \mathrm{s} .
$$

Mas, como $P V P=P$ então

$$
\operatorname{Tr}\left[P V P \frac{\partial V}{\partial \theta_{i}}\right]=y^{\prime} P \frac{\partial V}{\partial \theta_{i}} P y
$$

ou seja, o algorítmo iguala as formas quadráticas aos correspondentes valores esperados.

Como $V=\sum_{i=1}^{s} \frac{\partial V}{\partial \theta_{i}} \cdot \theta_{i}$ (veja ftem f), o resultado torna-se:

$$
\sum_{j=1}^{s} \operatorname{Tr}\left[P \frac{\partial V}{\partial \theta_{j}} P \frac{\partial V}{\partial \theta_{i}}\right] \theta_{j}=\left[y^{\prime} P \frac{\partial V}{\partial \theta_{i}} P y\right]
$$

em que s é o número de componentes a serem estimados.

As equação de (34) podem ser resumidas como

$$
B \theta=d
$$


para $B=b_{i j}$ e $d=d_{i} ; \mathrm{i}, \mathrm{j}=1, \ldots, \mathrm{s}$, com

$$
b_{i j}=\operatorname{Tr}\left[P \frac{\partial V}{\partial \theta_{j}} P \frac{\partial V}{\partial \theta_{i}}\right] \text { e } d_{i}=y^{\prime} P \frac{\partial V}{\partial \theta_{i}} P y .
$$

Observe que $\theta_{i}$ contém os componentes de covariancia para o erro ( $q(q+$ 1)/2 componentes) e os componentes dos fatores aleatórios inclurdos no modelo ( $\mathrm{r}$ fatores aleatórios com $r q(q+1) / 2+q^{2} r^{*}$ componentes). Então, segundo as considerações anteriores em (b) e em (c), tomando-se $R$ como a matriz dos componentes de covariancia para o erro e $G$ como a matriz de componentes de covariancia para todos fatores aleatórios, admitindose que os dados estão ordenados por características por individuos, tem-se, para as $\mathrm{N}$ observações:

$$
R=I \otimes E \quad \text { e } \quad G=A \otimes T
$$

Os elementos de $\theta$ são dados por:

$$
\boldsymbol{\theta}^{\prime}=\left[\begin{array}{llllllllll}
\sigma_{e_{1}}^{2} & \sigma_{e_{12}} & \cdots & \sigma_{e_{1 q}} & \cdots & \sigma_{e_{(q-1) q}} \sigma_{e_{q}}^{2} \sigma_{g_{11}}^{2} \sigma_{g_{12}} \cdots \sigma_{g_{1 q}} \cdots \sigma_{g_{r(q-1)}} \sigma_{g_{r q}}^{2}
\end{array}\right]
$$

Para $q=3$ e $r=2$, sem correlação entre os fatores aleatórios, tem-se 18 componentes de covariâncias. Ou seja:

$$
b_{e_{i j}}=\left[\begin{array}{c}
\operatorname{Tr}\left(P R_{i j} P R_{1}\right) \\
\operatorname{Tr}\left(P R_{i j} P R_{12}\right) \\
\vdots \\
\operatorname{Tr}\left(P R_{i j} P R_{13}\right) \\
\vdots \\
\operatorname{Tr}\left(P R_{i j} P R_{(23}\right) \\
\operatorname{Tr}\left(P R_{i j} P R_{3}\right)
\end{array}\right]
$$

$$
b_{g_{i j}}=\left[\begin{array}{c}
\operatorname{Tr}\left(P G_{i j} P R_{1}\right) \\
\operatorname{Tr}\left(P G_{i j} P R_{12}\right) \\
\vdots \\
\operatorname{Tr}\left(P G_{i j} P R_{13}\right) \\
\vdots \\
\operatorname{Tr}\left(P G_{i j} P R_{23}\right) \\
\operatorname{Tr}\left(P G_{i j} P R_{3}\right) \\
\operatorname{Tr}\left(P G_{i j} P G_{1}\right) \\
\operatorname{Tr}\left(P G_{i j} P G_{12}\right) \\
\vdots \\
\operatorname{Tr}\left(P G_{i j} P G_{13}\right) \\
\vdots \\
\operatorname{Tr}\left(P G_{i j} P G_{23}\right) \\
\operatorname{Tr}\left(P G_{i j} P G_{3}\right)
\end{array}\right]
$$


a) Derivação dos componentes de (co)variancia para os efeitos entre fatores aleatórios $\left(\hat{\sigma}_{g_{i j}}\right)$

Segundo HENDERSON (1984a e 1984b) $\operatorname{Var}(\hat{u})=G-C^{22}$, em que $C^{22}$ é uma sub-matriz da inversa generalizada da matriz de coeficientes $(C)$, isso é,

$$
\begin{gathered}
C^{-}=\left[\begin{array}{ll}
C^{11} & C^{12} \\
C^{12} & C^{22}
\end{array}\right] \text { e } \\
E\left(\hat{u}^{\prime} Q \hat{u}\right)=\operatorname{Tr}\left(Q\left(G-C^{22}\right)\right) .
\end{gathered}
$$

Conseqüentemente, associado com a $i^{\text {esima }}$ forma quadrática em $\hat{u}$, têm-se as equações: $\operatorname{Tr}\left(Q_{i} G\right)=\hat{u}^{\prime} Q_{i} \hat{u}+\operatorname{Tr}\left(Q_{i} C^{22}\right)$. O lado esquerdo de (36) é uma função linear de $\hat{\sigma}_{g_{i j}}$ e o lado direito é um escalar. No modelo genético aditivo, com parentesco entre individuos, as equações tornam-se:

$$
\begin{gathered}
\operatorname{Tr}\left(A^{-1} A \hat{\sigma}_{g_{i j}}\right)=\hat{u}_{i}^{\prime} A \hat{u}_{j}+\operatorname{Tr} A^{-1} C^{i j} \quad \text { ou } \\
\hat{\sigma}_{g_{i j}}=\frac{\hat{u}_{i}^{\prime} A^{-1} \hat{u}_{j}+\operatorname{Tr}\left(A^{-1} C^{i j}\right)}{n_{a_{i j}}} \quad(\mathrm{i} \leq \mathrm{j}=1, \ldots, \mathrm{q}) .
\end{gathered}
$$

Em que, $C^{i j}$ é uma submatriz de $C^{22}$ pertencente a $i^{\text {esima }}$ e $j^{\text {esima }}$ caracterf́sticas, $n_{a_{i j}}$ é o número de animais com observação para as características i e $\mathrm{j}$, e $A$ é a matriz de parentesco entre os indivíduos. Se o fator aleatório for independente ou o parentesco entre os animais for ignorado, ou não está disponfvel, então:

$$
\hat{\sigma}_{g_{i j}^{\prime}}=\frac{\hat{u}_{i}^{\prime} \hat{u}_{j}+\operatorname{Tr}\left(C^{i j}\right)}{n_{a_{i j}}} .
$$

b) Derivação dos Componentes do Erro ( $\left.\hat{\sigma}_{e_{i j}}\right)$

Conforme descrito em HENDERSON (1984a e 1984b)

$$
\begin{aligned}
W & =\left(X \vdots Z_{u}\right) \Rightarrow \operatorname{Var}(\hat{W})=\operatorname{Cov}\left(\hat{w}, \hat{w}^{\prime}\right) . \\
\hat{\varepsilon} & =y-X \hat{\beta}-Z \hat{u} . \\
\Rightarrow \operatorname{Cov}\left(\hat{\varepsilon}, \hat{\varepsilon}^{\prime}\right) & =\left(I-W C^{-} W^{\prime} R^{-1}\right) y=R-W C^{-} W^{\prime}=\operatorname{Var}(\hat{\varepsilon}) . \\
\text { Portanto, } \operatorname{Var}(\hat{\varepsilon}) & =R-W C^{-} W^{\prime} .
\end{aligned}
$$

Se todas características são observadas e os dados são ordenados por animais e por característica, as soluções para $\hat{\sigma}_{i j}$ são:

$$
\hat{\sigma}_{e_{i j}}=\frac{\left(\hat{\varepsilon}_{i}^{\prime} \hat{\varepsilon}_{j}+\operatorname{Tr}\left(H^{i j}\right)\right)}{N-r(X)}
$$


em que $H^{i j}$ é uma submatriz de $W C^{-} W^{\prime}$ pertencente a $\hat{\varepsilon}_{i}$ com $\hat{\varepsilon}_{j}$. Sendo $\hat{\varepsilon}_{i}$ o vetor de erros para a característica i e $\hat{\varepsilon}_{j}$ o vetor de erros para a característica $\mathbf{j}$.

Com valores perdidos, as equações para estimação de $\sigma_{e_{i j}}$ são:

$$
\operatorname{Tr}\left(Q_{i j} R\right)=\hat{\varepsilon}^{\prime} Q_{i j} \hat{\varepsilon}+\operatorname{Tr}\left(Q_{i j} W C^{-} W^{\prime}\right),
$$

em que $Q_{i j}$ é a matriz de formas quadráticas para estimar $\sigma_{e_{i j}}$, que muda a cada iteração. Também $W=(X \vdots Z)$ e $C^{-}$é uma inversa generalizada da matriz de coeficientes $(C)$, do modelo misto.

HARVILLE (1977) derivou fórmulas para estimação de componentes de covariancia que são mais rápidas do que as apresentadas em (38) e (37). VanRADEN (1986) as utilizou em análise univariada e VALENTE (1988) as estendeu para o caso multivariado. (veja VALENTE, 1988, GROENEVELD \& KOVAK, 1990). Pelas equações, os componentes de covariancia são obtidos como:

$$
\begin{aligned}
& \hat{\sigma}_{g_{i j}}^{(k+1)}=\left(\frac{\hat{u}_{i}^{\prime} A^{-1} \hat{u}_{j}}{n_{a_{i j}}-\operatorname{Tr}\left(A^{-1} C^{i j}\right) / \hat{\sigma}_{g_{i j}}^{(k)}}\right) \\
& \hat{\sigma}_{e_{i j}}^{(k+1)}=\left(\frac{\hat{\varepsilon}_{i}^{\prime} \hat{\varepsilon}_{j}}{N-\operatorname{Tr}\left(H^{i j}\right) / \hat{\sigma}_{\varepsilon_{i j}}^{(k)}}\right)
\end{aligned}
$$

Observe que pelo algoritmo EM os elementos inversos de uma submatriz de $C$, a matriz de coeficientes, são requeridos, o que para grandes problemas pode tornar-se de obtenção bastante diff́cil. Quando essa matriz é esparsa, o problema pode ser amenizado.

\section{Inversão Utilizando Matrizes Esparsas}

A inversa de uma matriz $\mathrm{B}$ de posto completo é definida como:

$$
B B^{-1}=I \text { ou } B B^{j}=i^{j}, B^{-1}=b^{j}, i=i^{j} \quad \mathrm{j}=1, \ldots, \mathrm{n}
$$

Um algorftmo geral, para inversão, trabalha em dois passos:

a) Fatoração da matriz de coeficientes em duas matrizes triangulares por meio da decomposição LU

$$
B=L U
$$

b) Cálculo da inversa da matriz $B$, para $j=1, \ldots, N$ :

$$
\begin{aligned}
& L d^{j}=i^{j} \Longleftrightarrow L^{-1} i^{j}, \text { coluna } \mathrm{j} \text { de } L^{-1} \mathrm{e} \\
& U b^{j}=d^{j} \Longleftrightarrow b^{j}=U^{-1} L^{-1} i^{j}=B^{-1} i^{j}, \text { coluna } \mathrm{j} \text { de } B^{-1}
\end{aligned}
$$


Os elementos dos vetores $i, d$ e $b$ jamais são requeridos ao mesmo tempo, necessitando-se, assim, de um único espaço fŕsico (MISZTAL, 1990). Para $B$ simética e positiva definida, a decomposição $L U$ pode ser substiturda com vantagens pela decomposição de Cholesky .

O programa MTC ("Multitrait REML Estimation") de MISZTAL (1993) utiliza o algorítmo EM e adota para a solução do sistema de equações o conjunto de rotinas FSPAK, para inversão da matriz de coeficientes $C$, que, em geral, é esparsa, principalmente na área de gado de leite.

Segundo DEMPSTER et al. (1977) e HENDERSON (1984a), os componentes de covariancia, adotando-se o algoritmo EM, são obtidos considerando-se que em cada iteração as esperanças das formas quadráticas são tomadas sobre a suposição de que $\tilde{G}=G$ e $\tilde{R}=R$, isso é, a solução é o valor do parâmetro.

\subsubsection{Método Livre de Derivadas}

Pelo método livre de derivadas, o processo de estimação dos componentes de covariância consiste em procurar valores para as variâncias e covariâncias tais que maximizem a função de verossimilhança restrita. Por este método, embora muito mais simples computacionalmente, o processo pode requerer muitas avaliações da verrossimilhança.

Seja a função de verossimilhança de $L^{\prime} y$ já derivada anteriormente:

$$
L_{2}=-\frac{1}{2}\left[\log |R|+\log |G|+\log |C|+y^{\prime} P y\right]
$$

em que $C$, de posto completo, é a matriz de coeficientes nas equações do modelo misto (MME), segundo o modelo em (31), e $P$ é a matriz projeção, mencionada em (e). $O$ cálculo de $\log |C|$ e $y^{\prime} P y$ requer a fatoração da matriz de coeficientes das MME. Essa é a parte em que há maior demanda computacional no processo de avaliação da verossimilhança (MEYER, 1993). Pelo método livre de derivadas, usualmente existem duas opções para se estimar os componentes de covariância a partir de $L_{2}$. A primeira opção consiste na absorção ou eliminação de Gauss, utilizando uma lista encadeada, para armazenar os coeficientes da matriz esparsa. Essa opção está disponivel nos programas DFREML de MEYER (1991 e 1993). Uma segunda opção consiste em se realizar a decomposição de Cholesky da matriz $C$, a matriz de coeficientes do modelo misto, utilizando um esquema comprimido de armazenamento dos elementos da matriz esparsa. Essa opção está disponivel nos programas DFREML de MEYER (1993) e MTDFREML de BOLDMAN et al. (1993), implementados com uso de rotinas para matrizes esparsas. Os outros dois termos necessários para $L_{2},|R|$ e $|G|$ podem ser avaliados diretamente.

Cálculo de $\log |C|$ e $y^{\prime} P y$

\section{a) Eliminação de Gauss}

Segundo GRASER et al. (1987), os termos $\log |C|$ e $y^{\prime} P y$ podem ser avaliados simultaneamente para todos modelos acomodados em (31), envolvendo a aplicação da eliminação de Gauss com pivoteamento diagonal (veja 2.1.1.2) da matriz: 


$$
M=\left[\begin{array}{lll}
y^{\prime} R^{-1} y & y^{\prime} R^{-1} X & y^{\prime} R^{-1} Z \\
X^{\prime} R^{-1} y & X^{\prime} R^{-1} X & X^{\prime} R^{-1} Z \\
Z^{\prime} R^{-1} y & Z^{\prime} R^{-1} X & Z^{\prime} R^{-1} Z+G^{-1}
\end{array}\right]
$$

em que $M$ é a matriz de coeficientes das equações do modelo linear misto (MME) aumentada pelo lado direito dessas equações e o vetor de formas quadráticas das observações, comumente referida como matriz do modelo misto (MMM).

A aplicação do método na matriz $M$, na prática, significa a absorção sequiencial de todas linhas e colunas em $y^{\prime} R^{-1} y$, o que resultará, ao final do processo, na obtenção de $y^{\prime}\left(V^{-1}-V^{-1} X\left(X^{\prime} V^{-1} X\right)^{-} X^{\prime} V^{-1}\right) y=y^{\prime} P y . \log |C|$ será calculado como a soma dos logarftmos dos elementos diagonais não nulos (pivôs não nulos) obtidos durante a absorção ou eliminação de Gauss.

\section{b) Decomposição de Cholesky}

A matriz de coeficientes pode ser decomposta no produto de uma matriz triangular inferior $L=\left[l_{i j}\right]$, com $l_{i j}=0$ para $\mathrm{j}>\mathrm{i}$ e sua transposta. $\mathrm{O}$ método assim denominado de decomposição de Cholesky requer que a matriz a ser decomposta seja positiva definida. Em consequienencia disso, uma submatriz de $C$ necessita ser considerada. Assim,

$$
L L^{\prime}=\left[\begin{array}{ll}
X^{\prime *} R^{-1} X^{*} & X^{* *} R^{-1} Z \\
Z^{\prime} R^{-1} X^{*} & Z^{\prime} R^{-1} Z+G^{-1}
\end{array}\right]
$$

Em que $X^{*}$ é uma matriz de posto coluna completo, obtida a partir da imposição de restrições não estimáveis na matriz original $X$.

Como o determinante de uma matriz triangular é simplesmente o produto dos seus elementos diagonais, então

$$
\log |C|=\log \left(\prod_{i=1}^{N R+N F^{*}} l_{i i}^{2}\right)=2 \sum_{i=1}^{N R+N F^{*}} \log \left(l_{i i}\right) .
$$

Tendo-se determinado a decomposição, as soluções para os efeitos fixos e aleatórios ajustados $w^{\prime}=\left(\beta^{*^{\prime}} u^{\prime}\right)$ ( $\operatorname{com} \beta^{*}$ representando o subvetor de $\beta$, pertencente a $X^{*}$ podem ser obtidas, resolvendo-se os dois sistemas triangulares:

$$
\boldsymbol{L} v=r \quad \mathrm{e} \quad \boldsymbol{L}^{\prime} w=v
$$

em que $v$ é definido como $L^{\prime} w$ e $r$ é um vetor de elementos do lado direito das MME. Devido à natureza triangular de $L$, este procedimento apresenta pouca demanda computacional, requerendo somente substituições "para frente" e retrosubstituições. As soluções podem então ser utilizadas, segundo HARVILLE (1977), BOLDMAN et al. (1993) e MEYER (1993), para determinar $y^{\prime} P y$, da seguinte forma:

$$
y^{\prime} P y=y^{\prime} R^{-1} y-y^{\prime} R^{-1} X^{*} \hat{\beta}^{*}-y^{\prime} R^{-1} Z \hat{u}
$$


Uma forma mais simples, mas equivalente, é mostrada por BOLDMAN et al. (1993). Para isso, seja $L L^{\prime}=C$, isto é:

$$
\left[\begin{array}{lll}
l_{11} & 0 & 0 \\
l_{21} & l_{22} & 0 \\
l_{31} & l_{32} & l_{33}
\end{array}\right]\left[\begin{array}{lll}
l_{11} & l_{21} & l_{31} \\
0 & l_{22} & l_{23} \\
0 & 0 & l_{33}
\end{array}\right]=\left[\begin{array}{lll}
c_{11} & c_{12} & c_{13} \\
c_{12} & c_{22} & c_{23} \\
c_{13} & c_{23} & c_{33}
\end{array}\right] .
$$

Para o sistema da forma

$$
C w=r,
$$

primeiramente encontra-se $L$, tal que $L L^{\prime}=C$. Então,

$$
L L^{\prime} w=r
$$

Seja $v=L^{\prime} w$, tal que $L v=r$. Por substituição "para frente" calcula-se $v$. Em seguida, por retrosubstituição, encontra-se $L^{\prime} w=v$. Obtido $w$, resulta que:

$$
y^{\prime} P y=y^{\prime} R^{-1} y-w r
$$

\section{Cálculo de $\log |R|$}

Seja $y$ ordenado conforme as características por classes de animais. Assuma que a correlação entre os erros entre características medidas em diferentes individuos seja nula. Isso faz com que $R$ seja bloco diagonal para animais. Dessa forma, $R=$ $I_{n_{d}} \oplus R_{i}=\left(\sum_{i=1}^{n_{d}}{ }^{+} R_{i}\right)$, com $n_{d}$ o número de animais com registro e $\oplus$ é o operador de soma direta. Para q características, existe um total de $W=2^{q}-1$ combinações de características medidas. Para $\mathrm{q}=2$, por exemplo, $\mathrm{W}=3$, com as combinações: característica 1, característica 2 e ambas (MEYER, 1991 e 1993, e MEYER et al., 1991 ). Para o indivíduo i que tem combinação de características w, $R_{i}$ é igual a $E_{w}$, a submatriz de $E$, obtida pela eliminação de linhas e colunas pertencentes aos registros perdidos. $O$ resultado será:

$$
\log |R|=\sum_{w=1}^{W} n_{w} \log \left|E_{w}\right|
$$

em que $n_{w}$ representa o número de animais com registros para a combinação de características w. Então a avaliação de $\log |R|$ requer o cálculo de W Log (determinantes) da matriz $E_{w}$ de dimensão $\leq \mathrm{q} \times \mathrm{q}$.

Cálculo de $\log |G|$

Considere um caso mais simples com um único efeito aleatório no modelo e o mérto genético aditivo para animal, representado por um vetor de dimensão q x $n_{a}$ ( $n_{a}$ o número total de animais $)$. Neste caso, $\mathrm{r}=1, r^{*}=0$ e $\mathrm{s}=\mathrm{q}(\mathrm{q}+1)$. Sejam os efeitos 
ordenados conforme as características por animais e seja $a_{i}$ um subvetor para o $i^{\text {ésimo }}$ animal com matriz de covariância $T$ ( $T$ com dimensão q x q) e seus elementos são as covariâncias genéticas aditivas. Então,

$$
\begin{aligned}
u^{\prime} & =a^{\prime}=\left(a_{1}^{\prime}, \ldots a_{n_{a}}^{\prime}\right) \\
G & =A \otimes T \\
\log |G| & =n_{a} \log |T|+q \log |A|
\end{aligned}
$$

em que $A$ é a matriz de coeficientes de parentesco entre indivíduos, e $\otimes$ representa o operador de produto direto ou produto de Kronecker. $\log |A|$ independe dos parâmetros a ser estimados, sendo desnecessária para maximizar $L_{2}$.

O modelo pode ser estendido para modelos mais complexos, por exemplo, com um segundo efeito aleatório $(\mathrm{m})$ para cada indivíduo, com subvetor $m_{i}\left(\mathrm{i}=1, \ldots, n_{a}\right)$, com mesma estrutura de correlação entre individuos. Um exemplo típico deste tipo de efeito é um efeito genético materno. Neste caso $\mathrm{r}=2$ e $T$ é de dimensão $2 \mathrm{q} \times 2 \mathrm{q}$, com s $=3 q(q+1) / 2$, se os dois efeitos aleatórios forem não correlacionados $\left(r^{*}=0\right)$ e $s=q(5 q+$ $3) / 2$, para $r^{*}=1$. Assumindo que $u$ encontra-se ordenado de acordo com os efeitos por classes de animais, isto é, $u^{\prime}=\left(a_{1}^{\prime} m_{1}^{\prime}, \ldots a_{n_{a}}^{\prime} m_{n_{a}}^{\prime}\right)$, então, obtêm-se os mesmos resultados de (45) e (46) para $T=\operatorname{Var}\left(a_{i}^{\prime} m_{i}^{\prime}\right)$. Freqüentemente, há interesse de incluir efeito aleatório adicional não correlacionado com animal no modelo de análise. Tal efeito (como neste trabalho) pode ser um efeito permanente de meio (efeito não genético aditivo) devido ao próprio animal, em um modelo de repetibilidade. Considere este efeito com NC nIveis por característica e vamos representá-lo como $P$. Então, a matriz $T$ pode ser partida em blocos diagonais com $T_{A}$ para efeito genético aditivo e $T_{P}$ para efeito adicional não correlacionado. Conseqüentemente (45) e (46) tornam-se:

$$
\begin{aligned}
u^{\prime} & =\left(\begin{array}{ll}
a^{\prime} & p^{\prime}
\end{array}\right) \\
G & =\operatorname{diag}\left(A \otimes T_{A} ; D \otimes T_{P}\right) \\
\log |G| & =n_{a} \log \left|T_{A}\right|+N C \log \left|T_{P}\right|+q(\log |A|+\log |D|)
\end{aligned}
$$

em que $D$, normalmente uma matriz identidade, descreve a estrutura de correlação entre nfveis de $P$. Novamente $\log |A|$ e $\log |D|$ são constantes e não necessitam ser avaliadas.

Considere agora um caso bem geral, para um modelo com efeitos genéticos direto $\left(G_{1}=A \sigma_{g}^{2}\right)$ e materno $\left(G_{2}=A \sigma_{m}^{2}\right)$ e efeito permanente de meio com p nfveis e variância $\sigma_{p}^{2}$. Neste caso,

$$
\begin{gathered}
G=\left[\begin{array}{ccc}
A \sigma_{g}^{2} & A \sigma_{g_{m}} & 0 \\
A \sigma_{g_{m}} & A \sigma_{m}^{2} & 0 \\
0 & 0 & I_{p} \sigma_{p}^{2}
\end{array}\right] . \text { Então, } \\
\log |G|=2 \log |A|+q \log \left|\begin{array}{cc}
\sigma_{g}^{2} & \sigma_{g_{m}} \\
\sigma_{g_{m}} & \sigma_{m}^{2}
\end{array}\right|+p \log \left(\sigma_{p}^{2}\right)
\end{gathered}
$$


$\log |A|$ é uma constante que não necessita ser calculada, mas se o for, pode-se também adotar a decomposição de Cholesky, ou seja $A=L L^{\prime} \Rightarrow \log |A|=$ $\log |L|+\log \left|L^{\prime}\right| \Rightarrow \log |A|=2 \sum \log \left(l_{i i}\right)$.

\section{Casos Especiais}

Frequentemente, características especiais da estrutura dos dados ou modelo de análise podem ser exploradas com o objetivo de reduzir os requerimentos computacionais, para avaliação da verossimilhança ou o número de avaliaçōes requeridas para localização do máximo da função (MEYER, 1993).

\section{a) Análise Univariada}

Para análise univariada, $R=\sigma_{E}^{2} I$ e a variância do erro pode ser estimada diretamente das somas de quadrados do resíduo, como

$$
\sigma_{E}^{2}=\frac{y^{\prime} P y}{(N-r(X))}
$$

Como consequiencia, a verossimilhança pode ser maximizada com relação aos parâmetros remanescentes, expressos como uma função das variâncias originais e de $\sigma_{E}^{2}$, isto é, a dimensão da procura é reduzida para um (GRASER et al., 1987 e MEYER, 1989).

\section{b) Igualdade de Matrizes de Delineamento entre Características}

Se as matrizes de delineamento adotadas no modelo linear são iguais para todas características, então (31) (pág. 77) pode ser reescrito como:

$$
y=\left(I_{q} \otimes X_{0}\right) \beta+\left(I_{q} \otimes Z\right) u+\varepsilon
$$

Neste caso assume-se que os dados são ordenados conforme os animais por características. Sejam $\mathrm{n}=\mathrm{N} / \mathrm{q}, \mathrm{nr}=\mathrm{NR} / \mathrm{q}$ e $\mathrm{nf}=\mathrm{NF} / \mathrm{q}$ o número de registros, niveis de efeitos aleatórios e niveis de efeitos fixos por característica, respectivamente. $X_{0}$ de ordem $\mathrm{n} \times \mathrm{nf}$ e $Z_{0}$ de ordem $\mathrm{n} \times \mathrm{nr}$ são então matrizes de delineamento para os efeitos fixos e aleatórios para cada característica, enquanto $I_{q}$ representa uma matriz identidade de ordem q. Desde que todos animais tenham registro para todas características, então $R$ toma a forma

$$
R=E \otimes I, \text { e } \log |R|=n \log |E|
$$

Considere agora a decomposição da covariância residual em $E=Q Q^{\prime}$ fornecendo $R^{-1}=$ $\left(Q^{\prime-1} \otimes I_{n}\right)\left(Q^{-1} \otimes I_{n}\right)$. Transformando o vetor de dados para $y^{*}=\left(Q^{-1} \otimes I_{n}\right) y$, a matriz $M$ de (43) é substituida por

$$
M^{*}=\left[\begin{array}{lll}
y^{\prime *} y^{*} & y^{\prime *}\left(I_{q} \otimes X_{0}\right) & y^{\prime *}\left(I_{q} \otimes Z_{0}\right) \\
\left(I_{q} \otimes X_{0}\right) y^{\prime *} & I_{q} X_{0}^{\prime} X_{0} & I_{q} \otimes X_{0}^{\prime} Z_{0} \\
\left(I_{q} \otimes Z_{0}^{\prime}\right) y^{*} & I_{q} \otimes Z_{q}^{\prime} X_{0} & I_{q} \otimes Z_{0}^{\prime} Z_{0}+\left(G^{*}\right)^{-1}
\end{array}\right]
$$




$$
\begin{aligned}
\operatorname{com} G^{*}=\left(Q^{-1} \otimes I_{n r}\right) G\left(Q^{\prime-1}\right. & \left.\otimes I_{n r}\right) \mathrm{e} \\
y^{\prime} P y & =y^{\prime *} P^{*} y^{*} ; \\
\log |C| & =\log \left|C^{*}\right|-(n r+n f) \log |E| \text { com } \\
C^{*} & =\left(Q^{\prime} \otimes I_{n f+n r}\right) C\left(Q \otimes I_{n f+n r}\right) . \quad \mathrm{e} \\
P^{*} & =\left(Q^{\prime-1} \otimes I_{n}\right) P\left(Q^{-1} \otimes I_{n}\right)
\end{aligned}
$$

\section{c) Transformação Canônica}

Quando existem observações para todas q características, pode-se utilizar a transformação canônica (veja (2.1.2.2), pág. 15). O uso de uma transformação canônica dos dados para estimar componentes de (co)variancia, pelo método REML, para $q$ características correlacionadas, produz um conjunto de q novas variáveis denominadas variáveis canônicas, que são genética e fenotipicamente não correlacionadas. Então, neste caso, pode-se transformar uma análise multivariada em uma série de q analises univariadas que resulta em uma substancial redução do esforço computacional (veja, entre outros, MEYER (1991) e VALENTE, 1988). Segundo MEYER (1993), para modelos nos quais se está ajustando efeito aleatório adicional, uma transformação canônica dos dados pode reduzir em até $60 \%$ o tempo para avaliação da função de verossimilhança.

Considere um modelo animal (MA) sem efeito aleatorio adicional, isto é, $u=a, T=\operatorname{Var}\left(a_{i}\right)$ e $G=T \otimes A$, com matriz de delineamento igual para todas caracterfsticas. Seja $\lambda_{i}$, para $i=1, \ldots, \mathrm{q}$ os autovalores de $E^{-1} T$ e $S$ a matriz de autovetores correspondentes. Então,

$$
S T S=\operatorname{diag}\left(\lambda_{i}\right) \text { e } S E S^{\prime}=I_{q}
$$

Isto é, $E=S^{-1} S^{\prime-1}, Q=S^{-1}$ e $G^{*}=\operatorname{diag}\left(\lambda_{i} A\right)$. Então $S$, com elementos $s_{i j}$, descreve a transformação canônica.

Para $y^{*}=\left(S \otimes I_{n}\right) y$, a matriz de variâncias do vetor de dados transforma$\operatorname{dos}, V^{*}=\operatorname{Var}\left(y^{*}\right)$, a matriz de coeficientes $C^{*}$ e matriz projeção $P^{*}$ são "bloco diagonal" para características. Conseqëntemente, $M^{*}$ pode ser partida em q matrizes independentes $M_{i}^{*}$

$$
M_{i}^{*}=\left[\begin{array}{lll}
y_{i}^{\prime *} y_{i}^{*} & y_{i}^{\prime *}\left(I_{q} \otimes X_{0}\right) & y_{i}^{\prime *}\left(I_{q} \otimes Z_{0}\right) \\
\left(I_{q} \otimes X_{0}\right) y_{i}^{\prime *} & I_{q} X_{0}^{\prime} X_{0} & I_{q} \otimes X_{0}^{\prime} Z_{0} \\
\left(I_{q} \otimes Z_{0}^{\prime}\right) y_{i}^{*} & I_{q} \otimes Z_{q}^{\prime} X_{0} & I_{q} \otimes Z_{0}^{\prime} Z_{0}+\lambda_{i}^{-1} A^{-1}
\end{array}\right]
$$

em que $y_{i}^{*}$ é um subvetor de $y^{*}$ para a característica $\mathrm{i}$.

Cada uma dessas submatrizes é equivalente à matriz de coeficientes em uma análise univariada de uma única característica com herdabilidade $\lambda_{i} /\left(\lambda_{i}+1\right)$. Absorvendo da matriz $M_{i}^{*}$ as linhas e colunas 2 até $\mathrm{k}=\mathrm{nf}+\mathrm{nr}+1$ (saltando as linhas com pivô nulo), em $y_{i}^{\prime *} y_{i}^{*}$, será produzida a forma quadrática $y^{\prime *} P_{i}^{*} y_{i}^{*}$ e $\log \left|C_{i}^{*}\right|$, em que $P_{i}^{*}$ e $C_{i}^{*}$ são submatrizes de $P^{*}$ e $C^{*}$, respectivamente, para a caracteristica i. Assim, as quantidades requeridas são obtidas como:

$$
y^{\prime} P y=\sum_{i=1}^{q} y_{i}^{\prime *} P_{i}^{*} y_{i}^{*}
$$




$$
\log |C|=\sum_{i=1}^{q} \log \left|C_{i}^{*}\right|-(n r+n f) \log |E|
$$

Os demais elementos da função de verossimilhança são obtidos de forma similar ao apresentado anteriormente.

\subsection{Estimação dos Parâmetros Genéticos, Fenotı́picos e de meio ambien- te}

- a) Estimativa do coeficiente de herdabilidade para a caracteristica i $\left(\hat{h}_{i}^{2}\right)$

$\left.a_{1}\right)$ Modelo Animal:

$$
\hat{h}_{i}^{2}=\frac{\hat{\sigma}_{a_{i}}^{2}}{\hat{\sigma}_{P_{i}}^{2}}
$$

a.) Modelo de Reprodutor

$$
\hat{h}_{i}^{2}=4 * \frac{\hat{\sigma}_{a_{i}}^{2}}{\hat{\sigma}_{P_{i}}^{2}}
$$

- b) Estimatiiva do coeficiente de repetibilidade para a caracteristica i $\left(\hat{r}_{i}\right)$

$$
\hat{r}_{i}=\frac{\hat{\sigma}_{a_{i}}^{2}+\hat{\sigma}_{p_{i}}^{2}}{\hat{\sigma}_{P_{i}}^{2}}
$$

- c) Estimativa do coeficiente $\hat{c}_{i}^{2}$ para a caracteristica $\mathrm{i}$

$$
\hat{c}_{i}^{2}=\frac{\hat{\sigma}_{p_{i}}^{2}}{\hat{\sigma}_{P_{i}}^{2}}
$$

- d) Estimativas dos coeficientes de correlação genética $\left(\hat{r}_{g}\right)$, fenotfpica $\left(\hat{r}_{p}\right)$ e de meio ambiente $\left(\hat{r}_{e}\right)$

$\left.\mathrm{d}_{1}\right)$ Correlação genética entre as caracterı́sticas i e j $\left(\hat{r}_{g_{i j}}\right)$

$$
\hat{r}_{g_{i j}}=\frac{\hat{\sigma}_{a_{i j}}}{\sqrt{\hat{\sigma}_{a_{i}}^{2} * \hat{\sigma}_{a_{j}}^{2}}}
$$

$\mathrm{d}_{2}$ ) Correlação fenotípica entre as características i e j $\left(\hat{r}_{p_{i j}}\right)$

$$
\hat{r}_{p_{i j}}=\frac{\hat{\sigma}_{P_{i j}}}{\sqrt{\hat{\sigma}_{P_{i}}^{2} * \hat{\sigma}_{P_{j}}^{2}}}
$$


$\mathrm{d}_{3}$ ) Correlação de meio ambiente entre as caracterı́sticas i e j $\left(\hat{\boldsymbol{r}}_{e_{\mathrm{ij}}}\right)$

$$
\hat{r}_{e_{i j}}=\frac{\hat{\sigma}_{e_{i j}}}{\sqrt{\hat{\sigma}_{e_{i}}^{2} * \hat{\sigma}_{e_{j}}^{2}}}
$$

em que:

$\hat{\sigma}_{a_{i}}^{2}$ em modelo animal, representa a estimativa da variância genética aditiva para a catacterística i. Em modelo de reprodutor (ou equivalente), representa o componente de variância de reprodutor ou $1 / 4$ da estimativa da variância genética aditiva para a caracteristica i;

$\hat{\sigma}_{p_{i}}^{2}$ é a estimativa da variância devido aos efeitos permanentes de meio para a caracteristica $\mathrm{i}$;

$\hat{\sigma}_{e_{i}}^{2}$ é a estimativa da variância devido aos efeitos de meio ambiente para a caracteŕstica i;

$\hat{\sigma}_{P_{i}}^{2}$ é a estimativa da variância fenotípica para a característica $\mathrm{i}\left(\hat{\sigma}_{P_{i}}^{2}=\hat{\sigma}_{a_{i}}^{2}+\hat{\sigma}_{p_{i}}^{2}+\hat{\sigma}_{e_{i}}^{2}\right)$;

$\hat{\sigma}_{a_{i j}}$ é a estimativa da covariância genética entre as características i e j;

$\hat{\sigma}_{p_{i j}}$ é a estimativa da covariancia para os efeitos permanentes de meio, entre as características i e j;

$\hat{\sigma}_{e_{i j}}$ é a estimativa da covariância para os efeitos de meio ambiente, entre as caracteristicas i e j.

$\hat{\sigma}_{P_{i j}}$ é a estimativa da covariância fenotı́pica, entre as características i e j.

$\hat{c}_{i}^{2}$ é a estimativa da proporção da variancia fenotípica que é devido aos efeitos permanentes de meio, para a caracteristica i. 


\section{RESULTADOS E DISCUSSÃO}

\subsection{Análise Univariada}

As tabelas de 8 a 10c mostram os resultados das análises univariadas dos dados dos arquivos UNIVA282.ARQ, SIMUL050.MIS e CNPGL001.ARQ.

Os valores estimados dos componentes de variancia $\left(\hat{\sigma}_{i}^{2}\right)$, herdabilidade $\left(\hat{h}^{2}\right)$, repetibilidade $(\hat{r})$ e coeficiente $\left(\hat{c}^{2}\right)$, para os três sistemas DFREML, MTC e MTDFREML, para as três amostras analisadas, são similares.

Para o modelo adotado, tipo 1 (sistemas DFREML e MTDFREML) ou tipo 2 (sistema MTC) (Tabelas de 7 a 7b), em que inclui os efeitos fixos e efeitos aleatórios de animal mais efeito permanente de meio, acrescido do erro (Tabelas 8 e 10), constata-se constância dos valores estimados frente a diferentes valores iniciais.

Os valores iniciais adotados nas análises referentes à tabela 8 variam de 0.1 a 10 vezes os valores iniciais apresentados na tabela. Por observar constancia das estimativas sob diversos valores iniciais, apresentam-se apenas os resultados correspondentes a valores iniciais próximos aos valores das estimativas. Em razão do processo de procura do máximo da função de verossimilhança ser unidimensional (análise univariada) e os efeitos aleatórios serem independentes entre si, a convergência é rápida e as estimativas dos parâmetros são similares, sob diferentes valores iniciais.

Esse resultado não pode ser generalizado para qualquer tipo de modelo. BOLDMAN et al. (1993) adotam um modelo incluindo efeito aleatorio adicional correlacionado com animal (efeito materno) e observam variações nos valores estimados, inclusive falsa convergência ou convergência para máximos locais, frente a diferentes razões de variancias $\theta_{a}, \theta_{m}, \theta_{a m}$ e $\theta_{c}$. Em que $\theta_{a}=\frac{\sigma_{a}^{2}}{\sigma_{p}^{2}}, \theta_{m}=\frac{\sigma_{m}^{2}}{\sigma_{p}^{2}}, \theta_{a m}=\frac{\sigma_{a m}^{2}}{\sigma_{p}^{2}}, \theta_{c}=\frac{\sigma_{c}^{2}}{\sigma_{p}^{2}}$ e $\sigma_{p}^{2}=$ $\sigma_{a}^{2}+\sigma_{m}^{2}+\sigma_{a m}^{2}+\sigma_{c}^{2}+\sigma_{e}^{2}$. Naquele mesmo trabalho verifica-se, em alguns casos, falsa convergência pelo método simplex, mesmo após repetições sucessivas do processo de estimação.

A análise dos dados do arquivo SIMUL050.MIS sob o enfoque univariado (Tabela 9), também mostra que as estimativas obtidas pelos diferentes sistemas são praticamente as mesmas. As pequenas diferenças encontradas podem ser atriburdas a problemas de amostragem, decorrente de tamanho reduzido da amostra, e a diferentes niveis de precisão adotados em cada um dos sistemas. 
Em geral, há alta velocidade de convergência para os diferentes sistemas. Esse resultado deve-se basicamente à adoção de técnicas numéricas adequadas nos sistemas e, sobretudo, à esparsividade das MME. Nestas análises o número relativo de elementos não nulos nas MME é muito pequeno, de forma que a ordenação e fatoração das equações são realizadas de forma rápida, com pouca demanda de memória RAM.

Os programas do sistema DFREML (DFUNI, DFMUV e DFMUW) oferecem a opção para operação com ou sem matriz esparsa, quando da escolha do processo de ordenação das equações (opção -2). Desta forma, segundo a conveniência, fica a critério do usuário adotar ou não tal estratégia, no processo de estimação. Em geral, em análise de dados de campo em rebanhos leiteiros, é vantajoso fazer a ordenação das equações trabalhando-se com matrizes esparsas.

Embora a convergência tenha sido relativamente rápida em análise univariada, verificam-se algumas diferenças à convergência entre os sistemas. Estas são observadas no tempo gasto no processo de estimação, no número de iterações e no número de avaliações da verossimilhança. Em geral o sistema MTDFREML apresenta performance inferior nesses itens. Isso em realidade é esperado, pois o programa MTDFRUN, parte desse sistema, é um programa geral que visa analisar qualquer tipo de modelo linear uni ou multivariado, com mesmo ou diferente tipo de modelo por variável. Além disso, o critério de convergência adotado é mais rigoroso $\left(\operatorname{Var}\left(-2 \log \left(\mathrm{L}_{2}\right)<10^{-9}\right.\right.$ para MTDFREML x $10^{-8}$ para o DFREML). Por outro lado, o sistema DFREML (Tabelas 1 e 7 ) é modularizado, existindo um programa para análise univariada (ou análise multivariada com igualdade de matriz de delineamento) (DFUNI), outro programa para análise multivariada com mesmo modelo de análise para todas características (DFMUV) e outro para análise multivariada com diferentes modelos de análise para as características (DFMUW). O sistema MTC é mais simples, restrito e compacto (veja Tabelas 3 e 7a.). Além disso, ele adota um algorítmo EM que, por natureza, espera-se que seja mais rápido quando há convergência regular.

O sistema DFREML não executa análises de modelos onde não se disponha de informação de parentesco entre os animais, ou seja, quando todos pais e mães no arquivo de pedigree apresentam número 0 . Isso mostra que o sistema foi implementado dentro da filosofia de análise considerando um modelo animal, exigindo que haja pelo menos uma informação que possa relacionar animais.

As tabelas de 10 a 10c mostram os resultados das análises univariadas dos dados de campo do arquivo CNPGL001.ARQ utilizando-se um modelo animal ou modelo de reprodutor. Para o modelo de reprodutor, não se dispõe de informação da paternidade para os touros $(A=I)$, ou seja, os pais e mães dos reprodutores são considerados desconhecidos.

A média fenotípica de 3699 registros de produção de leite até 305 dias de lactação foi de $2358.26 \pm 11.5 \mathrm{~kg}$ e coeficiente de variação de $29.66 \%$. A tabela 10 mostra a análise considerando-se todos registros de produção como uma única variável, excluindo-se aqueles registros cuja vaca apresenta pai desconhecido. Para a tabela 10a. consideram-se as produções de leite no $1^{\circ}, 2^{\circ}$ e e $3^{\circ}$ partos como três características diferentes e as análises são executadas uma variável por vez, como se fosse de uma análise univariada comum. Nesse caso os dados são analisados adotando-se um modelo animal. O mesmo modelo, 
incluindo-se apenas os efeitos de ordem de parição e efeito permanente de meio, devido a registros repetidos de uma mesma vaca é adotado para os dados totais, cujos resultados são apresentados na tabela 10c.

Essas análises são realizadas principalmente para mostrar a adequação dos diversos programas aplicados a uma amostra de dados reais, cuja estrutura possivelmente pode diferir de estruturas de amostras simuladas. Embora acredite-se tratar de uma subamostra representativa da raça, não se fará, para esses dados, explorações sob o enfoque zootécnico, porque foge aos objetivos deste trabalho. Espera-se, no entanto, que a partir dos resultados obtidos, se possa recomendar uma análise dos dados para os mesmos parâmetros, com o total de dados atualizados do arquivo zootécnico nacional, para a raça Gir, adotando-se os programas utilizados.

Em geral as estimativas dos parâmetros genéticos são as mesmas ou similares em análise univariada, tanto para o modelo de reprodutor $\left(\hat{h}^{2}=0.14\right)$, quanto para o modelo animal $\left(\hat{h}^{2}=0.12 \pm 0.04\right)$.

Adotando-se o método livre de derivadas (amostra desbalanceada), as estimativas do coeficiente de herdabilidade da produção de leite até 305 dias de lactação são as mesmas para o dados do $1^{\circ}$ e do $3^{\circ}$ partos, que diferem do valor estimado para o $2^{\circ}$ parto. Esse resultado sugere que a maior pressão de seleção está sendo praticada no primeiro parto, reduzindo, desta forma, a proporção da variância genética aditiva no segundo parto.

Para a amostra aqui considerada, quando adota-se os dados balanceados, com apenas 763 registros por caracteristica, pela eliminação de registros onde alguma característica apresenta valor perdido, isto é, utilização dos dados em que todas vacas produzem nos três partos considerados (sistema MTC), as estimativas de herdabilidade são crescentes. Passando de 0.04 no $1^{\circ}$ parto, 0.06 no $2^{\circ}$ e para 0.25 no $3^{\circ}$ parto.

A redução da estimativa de herdabilidade do primeiro para o segundo parto é explicada pela alta taxa de descarte de matrizes que é praticada com base na baixa produção de leite no primeiro parto. MEYER (1983) encontra redução da estimativa de herdabilidade do $2^{\circ}$ para $3^{\circ}$ parto, mas não do $1^{\circ}$ para $2^{\circ}$ parto.

Observando-se a tabela 10a., notam-se grandes diferenças de valores estimados dos componentes de variancia e nas estimativas de herdabilidade de cada caracteristica pelos programas que adotam o método livre de derivadas e o método EM-REML ou programa MTC. A análise para o programa MTC refere-se apenas aos registros de produção no $1^{\circ}, 2^{\circ}$ e $3^{\circ}$ partos onde não se observa valor perdido. Para os demais programas, são analisados todos os dados amostrados. Essas diferenças são decorrência da amostra utilizada, e não de deficiência em si do programa MTC, pois os resultados da análise com a mesma subamostra de 763 registros são confirmados nos sistemas DFREML e MTDFREML. Na realidade, essa análise foi executada nos dados do arquivo preparado para análise multivariada e analisa-se cada uma das caracterfsticas como se fosse de uma análise univariada padrão. No entanto, nota-se aqui uma deficiência do sistema MTC, em análise multivariada de dados desta natureza (com valores perdidos). Assim, em casos de perda de registro, deve-se procurar realizar a análise aproveitando-se o número máximo de observações por característica avaliada, especialmente em situações de amostras pequenas.

Nessas análises univariadas, verifica-se que os métodos livres de derivadas 
em geral requerem apenas duas tentativas para que se considere o final do processo de estimação. Normalmente, a segunda tentativa confirma a primeira. Além disso, em termos de valores estimados, para modelos com um único fator aleatório além do erro, os sistemas são equivalentes.

As estimativas de herdabilidade da produção de leite, em análise univariada, para os dados do arquivo CNPGL001.ARQ, são baixas quando comparadas aos resultados encontrados na literatura, como aqueles citados e estimados por VERNEQUE(1982) em um rebanho Gir leiteiro, no entanto, as estimativas são similares aos resultados observados por RAMOS (1979), em análise de dados de vários rebanhos da mesma raça.

Tabela 8. Estimativas dos componentes de variância, do coeficiente de herdabilidade $\left(\hat{h}^{2}\right)$ e $\hat{c}^{2}$ para os dados do arquivo UNIVA282. ARQ, utilizando-se os programas DFUNI, MTDFRUN e MTC. Análise univariada - modelo animal

\begin{tabular}{|c|c|c|c|c|c|c|}
\hline \multirow{2}{*}{$\begin{array}{l}\text { Para- } \\
\text { metro }\end{array}$} & \multirow{2}{*}{$\begin{array}{l}\text { Valores } \\
\text { Iniciais }\end{array}$} & \multicolumn{2}{|c|}{ DFUNI } & \multirow{2}{*}{$\begin{array}{l}\text { MTDFRUN } \\
\text { Simplex }\end{array}$} & \multirow{2}{*}{$\begin{array}{r}\text { MTC } \\
\text { EM }\end{array}$} & \multirow{2}{*}{$\begin{array}{l}\text { (1) } \\
\text { GE }\end{array}$} \\
\hline & & Simplex & Powell & & & \\
\hline$\overline{\sigma_{a}^{2}}$ & 30 & 30,91 & 30,94 & 30,90 & 31,17 & 30,98 \\
\hline$\sigma_{c}^{2}$ & 15 & 15,08 & 14,94 & 14,94 & 14,89 & 14,88 \\
\hline$\sigma_{e}^{2}$ & 50 & 50,80 & 50,35 & 50,34 & 50,23 & 50,34 \\
\hline$h^{2}$ & 0,33 & 0,32 & 0,32 & 0,32 & 0,32 & 0,32 \\
\hline$c^{2}$ & 0,16 & 0,15 & 0,15 & 0,15 & 0,15 & 0,15 \\
\hline \multicolumn{7}{|c|}{ Informações Adicionais } \\
\hline \multirow{4}{*}{\multicolumn{2}{|c|}{$\begin{array}{l}\text { Tempo(s) } \\
\text { № de Iterações } \\
\text { № de Avaliações } \\
\text { Tentativas }\end{array}$}} & 58 & 72 & 81 & 40 & - \\
\hline & & 26 & 3 & 53 & 40 & 36 \\
\hline & & 51 & 24 & 104 & - & 74 \\
\hline & & 2 & 1 & 2 & 1 & 2 \\
\hline \multicolumn{2}{|c|}{$-2 \log \left(L_{2}\right)$} & 1509,55 & 1509,55 & 1509,55 & 1509,55 & 1509,55 \\
\hline \multicolumn{2}{|c|}{$\operatorname{Var}\left(-2 \log \left(\mathrm{L}_{2}\right)\right)$} & $<10^{-8}$ & $<10^{-8}$ & $<10^{-9}$ & $* *$ & $10^{-8}$ \\
\hline \multicolumn{2}{|c|}{ № de Equações } & 345 & 345 & 345 & 345 & 345 \\
\hline \multicolumn{2}{|c|}{ No de Registros } & 282 & 282 & 282 & 282 & 282 \\
\hline \multicolumn{7}{|c|}{ Elementos não } \\
\hline \multicolumn{2}{|c|}{ nulos nas $M M E$} & 344 & 344 & 2744 & - & - \\
\hline \multirow{2}{*}{\multicolumn{2}{|c|}{$\frac{\text { Esparsividade (\%) }}{(1)-\text { Resultados de } M}$}} & 0,6 & 0,6 & 4,3 & - & - \\
\hline & & ER (1989) & & ** - ConvG $<$ & $0^{-6}$ e Cor & $<10^{-6}$ \\
\hline \multicolumn{4}{|c|}{ GE - Eliminação de Gauss } & $\mathrm{EM}-\mathrm{M}$ & & beranças \\
\hline
\end{tabular}


Tabela 9. Estimativas dos componentes de variancia e do coeficiente de herdabilidade $\left(\hat{h}^{2}\right)$ para os dados do arquivo SIMUL050.MIS utilizando-se os programas DFUNI, MTDFRUN e MTC. Análise univariada - modelo animal

\begin{tabular}{|c|c|c|c|c|c|}
\hline \multirow{2}{*}{$\begin{array}{l}\text { Parâ- } \\
\text { metro }\end{array}$} & \multirow{2}{*}{$\begin{array}{l}\text { Valores } \\
\text { Iniciais }\end{array}$} & \multicolumn{2}{|c|}{ DFUNI } & \multirow{2}{*}{$\begin{array}{c}\text { MTDFRUN } \\
\text { Simplex }\end{array}$} & \multirow{2}{*}{$\begin{array}{r}\text { MTC } \\
\text { EM } \\
\end{array}$} \\
\hline & & Simplex & Powell & & \\
\hline$\sigma_{a_{11}}^{2}$ & 173000 & 169078 & 169101 & 169263 & 169257 \\
\hline$\sigma_{a_{22}}^{2}$ & 300 & 217,96 & 218,09 & 218,39 & 214,99 \\
\hline$\sigma_{a_{33}}^{2}$ & 190 & 141,24 & 141,45 & 141,46 & 139,59 \\
\hline$\sigma_{e_{11}^{2}}^{2}$ & 415000 & 417493 & 417508 & 417519 & 417369 \\
\hline$\sigma_{e_{22}}^{2}$ & 508 & 644,78 & 644,38 & 644,52 & 647,49 \\
\hline$\sigma_{e_{33}^{2}}^{2}$ & 332 & 329,09 & 329,00 & 328,09 & 331,09 \\
\hline$h_{1}^{2}$ & 0,29 & 0,29 & 0,29 & 0,29 & 0,29 \\
\hline$h_{2}^{2}$ & 0,37 & 0,25 & 0,25 & 0,25 & 0,25 \\
\hline$h_{3}^{2}$ & 0,36 & 0,30 & 0,30 & 0,30 & 0,30 \\
\hline \multicolumn{6}{|c|}{ Informações Adicionais } \\
\hline \multirow{5}{*}{\multicolumn{2}{|c|}{$\begin{array}{l}\text { Tempo(s) }{ }^{+} \\
\text {№ de Iterações } \\
\text { № de Avaliações } \\
\text { Tentativas } \\
-2 \log \left(\mathrm{L}_{2}\right)\end{array}$}} & 33 & 31 & 204 & 20 \\
\hline & & 28 & 2 & 28 & 18 \\
\hline & & 34 & 27 & 61 & - \\
\hline & & 2 & 2 & 2 & 1 \\
\hline & & 2376,18 & 2376,18 & 2376,18 & 2376,19 \\
\hline & & 1315,65 & 1315,65 & 1315,65 & 1315,16 \\
\hline \multirow{2}{*}{\multicolumn{2}{|c|}{$\operatorname{Var}\left(-2 \log \left(\mathrm{L}_{2}\right)\right)$}} & 1213,31 & 1213,31 & 1213,31 & 1213,31 \\
\hline & & $<10^{-8}$ & $<10^{-8}$ & $<10^{-9}$ & $* *$ \\
\hline \multicolumn{2}{|c|}{ № de Equações } & 211 & 211 & 211 & 200 \\
\hline \multicolumn{2}{|c|}{ № de Registros } & 204 & 204 & 204 & 204 \\
\hline \multicolumn{2}{|c|}{ Elementos não } & & & & \\
\hline \multicolumn{2}{|c|}{ nulos nas MME } & 199 & 199 & 1923 & - \\
\hline \multicolumn{2}{|c|}{ Esparsividade(\%) } & 1 & 1 & 8,16 & - \\
\hline \multicolumn{2}{|c|}{ Class. Ef. Fixos } & 42 & 42 & 42 & 42 \\
\hline \multicolumn{2}{|c|}{ Animais S/Reg. } & 41 & 41 & 41 & 30 \\
\hline \multicolumn{2}{|c|}{ Animais C/Reg. } & 128 & 128 & 128 & 128 \\
\hline
\end{tabular}


Tabela 10. Estimativas dos componentes de variancia e dos coeficientes de herdabilidade $\left(\hat{h}^{2}\right)$ e repetibilidade $(\hat{r})$ para os dados do arquivo CNPGL001.ARQ, utilizando-se os programas MTDFRUN e MTC. Análise univariada - modelo de reprodutor

\begin{tabular}{|lrrr|}
\hline Parâ- & Valores & \multicolumn{2}{c|}{ Valores Estimados } \\
\cline { 2 - 4 } metro & Iniciais & MTDFRUN & MTC \\
\hline$\sigma_{a}^{2}$ & 8800 & 12030,06 & 12038,39 \\
$\sigma_{p}^{2}$ & 145000 & 154772,13 & 154723,45 \\
$\sigma_{e}^{2}$ & 200000 & 169774,87 & 169787,06 \\
\hline$h^{2}$ & 0,10 & 0,14 & 0,14 \\
$r$ & 0,43 & 0,46 & 0,46 \\
\hline & Informações & Adicionais & \\
\hline Tempo(s) & & 855 & 138 \\
№ de Iterações & & 92 & 16 \\
№ de Avaliações & & 165 & - \\
No de Tentativas & & 2 & 1 \\
-2Log(L $\left.{ }_{2}\right)$ & 40972,80 & 40972,80 \\
Var(-2Log(L 2$)$ ) & $<10^{-9}$ & $* *$ \\
№ de Registros & 3307 & 3307 \\
№ de Equações & 1982 & 1982 \\
Elementos não & & \\
nulos nas M M E & & 28164 & - \\
Esparsividade(\%) & 1,38 & - \\
Class. de Ef. Fixo. & 333 & 333 \\
Animais S/Registros & 152 & 152 \\
Animais C/Registros & 1497 & 1497 \\
\hline
\end{tabular}

** - ConvG $<10^{-6}$ e ConvR $<10^{-6}$ 
Tabela 10a. Estimativas dos componentes de variância e coeficiente de herdabilidade $\left(\hat{h}^{2}\right)$ para os dados do arquivo CNPGL001.ARQ utilizando-se os programas DFUNI, MTC e MTDFRUN. Análise univariada da produção de leite no $1^{\circ}\left(P_{1}\right), 2^{\circ}\left(P_{2}\right)$ e no $3^{\circ}\left(P_{3}\right)$ partos. Modelo animal

\begin{tabular}{|c|c|c|c|c|c|}
\hline Característica & $\begin{array}{l}\text { Pará- } \\
\text { metro }\end{array}$ & $\begin{array}{l}\text { Valores } \\
\text { Iniciais }\end{array}$ & DFUNI & MTC & MTDFRUN \\
\hline $\begin{array}{l}\text { Produção de } \\
\text { Leite no } 1^{\circ} \\
\text { Parto }\left(P_{1}\right)\end{array}$ & $\begin{array}{l}\sigma_{a_{1}}^{2} \\
\sigma_{e_{1}}^{2} \\
h_{1}^{2} \\
\text { ep }\end{array}$ & $\begin{array}{r}50000 \\
300000 \\
-\end{array}$ & $\begin{array}{r}43753 \\
203019 \\
0,18 \\
0,08\end{array}$ & $\begin{array}{r}8659 \\
220204 \\
0,04 \\
-\end{array}$ & $\begin{array}{r}43797 \\
203092 \\
0,18 \\
-\end{array}$ \\
\hline $\begin{array}{l}\text { Produção de } \\
\text { Leite no } 2^{\circ} \\
\text { Parto }\left(P_{2}\right)\end{array}$ & $\begin{array}{l}\sigma_{a_{2}}^{2} \\
\sigma_{e_{2}}^{2} \\
h_{2}^{2} \\
\text { ep }\end{array}$ & $\begin{array}{r}50000 \\
300000 \\
-\end{array}$ & $\begin{array}{r}29158 \\
348322 \\
0,08 \\
0,08\end{array}$ & $\begin{array}{r}20879 \\
336098 \\
0,06 \\
-\end{array}$ & $\begin{array}{r}29187 \\
348232 \\
0,08 \\
-\end{array}$ \\
\hline $\begin{array}{l}\text { Produção de } \\
\text { Leite no } 3^{\circ} \\
\text { Parto }\left(P_{3}\right)\end{array}$ & $\begin{array}{l}\sigma_{a_{3}}^{2} \\
\sigma_{e_{3}}^{2} \\
h_{3}^{2} \\
\text { ep }\end{array}$ & $\begin{array}{r}50000 \\
300000\end{array}$ & $\begin{array}{r}70545 \\
348241 \\
0,17 \\
0,10\end{array}$ & $\begin{array}{r}102342 \\
305987 \\
0,25 \\
-\end{array}$ & $\begin{array}{r}70626 \\
348102 \\
0,17 \\
-\end{array}$ \\
\hline
\end{tabular}

$\mathrm{EP}=$ Erro Padrão

Tabela 10b. Informações adicionais para os dados da tabela $10 \mathrm{a}$ referentes à análise univariada do arquivo CNPGL001.ARQ pelos programas DFUNI, MTC e MTDFRUN

\begin{tabular}{|l|rrr|rrr|rrr|}
\hline \multirow{2}{*}{ Informação } & \multicolumn{3}{|c|}{ DFUNI } & \multicolumn{3}{c|}{ MTC } & \multicolumn{3}{c|}{ MTDFRUN } \\
\cline { 2 - 10 } & \multicolumn{2}{|c|}{ Características } & \multicolumn{3}{c|}{ Caracteŕsticas } & \multicolumn{3}{c|}{ Características } \\
& $P_{1}$ & $P_{2}$ & $P_{3}$ & $P_{1}$ & $P_{2}$ & $P_{3}$ & $P_{1}$ & $P_{2}$ & $P_{3}$ \\
\hline Tempo(s) & 221 & 150 & 131 & 898 & 455 & 17 & 354 & 252 & 174 \\
№ de Iterações & 12 & 12 & 12 & 313 & 159 & 3 & 44 & 44 & 42 \\
№ de Avaliações & 15 & 15 & 16 & - & - & - & 88 & 88 & 85 \\
Tentativas & 2 & 2 & 2 & 1 & 1 & 1 & 2 & 2 & 2 \\
№ de Equações & 2491 & 1751 & 1321 & 1215 & 1215 & 1215 & 2668 & 1965 & 1544 \\
№ de Registros & 1718 & 1142 & 839 & 763 & 763 & 763 & 1718 & 1142 & 839 \\
Esparsividade das & & & & & & & & & \\
MME (\%) & 0,08 & 0,11 & 0,15 & - & - & - & 0,47 & 0,61 & 0,75 \\
№Clas.Ef.Fixos & 303 & 267 & 227 & 223 & 223 & 223 & 303 & 267 & 227 \\
Ordem das MM & 2489 & 1749 & 1319 & 1215 & 1215 & 1215 & 2668 & 1965 & 1544 \\
Animais S/Reg. & 470 & 341 & 254 & 229 & 229 & 229 & 647 & 556 & 478 \\
Animais C/Reg. & 1718 & 1142 & 839 & 763 & 763 & 763 & 1718 & 1142 & 839 \\
\hline
\end{tabular}


Tabela 10c. Estimativas dos componentes de variancia, coeficientes de herdabilidade $\left(\hat{h}^{2}\right), \hat{c}^{2}$ e repetibilidade $(\hat{r})$ para os dados do arquivo CNPGL001.ARQ, utilizando-se os programas DFUNI, MTDFRUN e MTC.Análise univariada

Modelo animal

\begin{tabular}{|c|c|c|c|c|c|}
\hline \multirow{3}{*}{$\begin{array}{l}\text { Para- } \\
\text { metro }\end{array}$} & \multirow{3}{*}{$\begin{array}{l}\text { Valores } \\
\text { Iniciais }\end{array}$} & \multicolumn{4}{|c|}{ Valores Estimados } \\
\hline & & \multicolumn{2}{|c|}{ DFUNI } & MTDFRUN & MTC \\
\hline & & Simplex & Powell & Simplex & EM \\
\hline$\overline{\sigma_{a}^{2}}$ & 50000 & 39982,64 & 40137,48 & 40019,23 & 40567,03 \\
\hline$\sigma_{c}^{2}$ & 100000 & 115417,05 & 115304,91 & 115423,13 & 114919,31 \\
\hline$\sigma_{e}^{2}$ & 200000 & 174618,61 & 174578,21 & 174599,10 & 174583,09 \\
\hline$h^{2}$ & - & $0,12 \pm 0,04$ & 0,12 & 0,12 & 0,12 \\
\hline$c^{2}$ & - & $0,35 \pm 0,04$ & 0,35 & 0,35 & 0,35 \\
\hline$r$ & - & $0,47 \pm 0,04$ & 0,47 & 0,47 & 0,47 \\
\hline \multicolumn{6}{|c|}{ Informações Adicionais } \\
\hline \multirow{6}{*}{\multicolumn{2}{|c|}{$\begin{array}{l}\text { Tempo(s) } \\
\text { № de Ierações } \\
\text { № de Avaliações } \\
\text { Tentativas } \\
-2 \log \left(\mathrm{L}_{2}\right) \\
\operatorname{Var}\left(-2 \log \left(\mathrm{L}_{2}\right)\right)\end{array}$}} & 723 & 542 & 1263 & 892 \\
\hline & & 32 & 5 & 80 & 51 \\
\hline & & 66 & 42 & 147 & - \\
\hline & & 2 & 2 & 2 & 1 \\
\hline & & 46207,80 & 46207,80 & 46207,80 & 46207,80 \\
\hline & & $<10^{-9}$ & $<10^{-9}$ & $<10^{-9}$ & $* *$ \\
\hline \multicolumn{2}{|c|}{$N^{\circ}$ de Equações } & 4249 & 4249 & 4426 & 4248 \\
\hline \multicolumn{2}{|c|}{ № de Registros } & 3699 & 3699 & 3699 & 3699 \\
\hline \multicolumn{2}{|c|}{ Elementos não } & & & & \\
\hline \multirow{2}{*}{\multicolumn{2}{|c|}{$\begin{array}{l}\text { nulos nas MME } \\
\text { Esparsividade }\end{array}$}} & 4246 & 4246 & 54816 & - \\
\hline & & & & & \\
\hline \multicolumn{2}{|c|}{ das MмE (\%) } & 0,05 & 0,05 & 0,54 & - \\
\hline \multicolumn{2}{|c|}{ Class. Ef. Fixo } & 343 & 343 & 343 & 343 \\
\hline \multicolumn{2}{|c|}{ Animais S/Reg } & 470 & 470 & 647 & 469 \\
\hline \multicolumn{2}{|c|}{ Animais C/Reg. } & 1718 & 1718 & 1718 & 1718 \\
\hline
\end{tabular}

\subsection{Análise Multivariada}

As tabelas de 11 até $20 \mathrm{a}$. mostram os resultados das análises dos dados dos diferentes arquivos citados na tabela 6, adotando-se análise multivariada e os sistemas DFREML, MTC e MTDFREML.

$\mathrm{Na}$ tabela 11 apresentam-se os resultados das análises do arquivo D221, adotando-se um modelo de reprodutor, sem informação de parentesco entre estes(modelo número 1 das tabelas $7 \mathrm{a}$. e 7b.). Os valores iniciais adotados na primeira análise foram obtidos por VALENTE(1988), que utilizou o método REMLl e o algorftmo Short Cut (SHC). Os dados desse arquivo são analisados apenas pelos sistemas MTDFREML e 
MTC. Adotam-se diferentes valores iniciais nas análises para verificar a sensibilidade do processo de estimação sob estas condições. As estimativas à convergência são invariantes sob diferentes valores iniciais. Por outro lado, conforme é apresentado na parte inferior da tabela 11, intitulada "informações adicionais", o processo de estimação adotando-se os dois sistemas mostra-se sensivel quanto ao tempo, número de iterações, número de avaliações da verossimilhança e tentativas, e número de vetores de parâmetros inválidos, sob diferentes valores iniciais.

Valores iniciais 10 vezes aos considerados ideais para as matrizes $G$ (de variância genética aditiva) e $R$ (de efeitos residuais) resultam em maior decréscimo de performance do processo, para o sistema MTDFREML. O mesmo não se observa para o programa MTC que apresenta-se sensivel apenas a variações em valores iniciais para a matriz $G$ ou para a matriz $R$, individualmente. Para o método livre de derivadas, nota-se acréscimo substancial no tempo e no número de iterações, de avaliações da verossimilhança e de vetores de parâmetrosinválidos, quando os valores iniciais utilizados são distantes das estimativas. Além disso, o processo de estimação apresenta-se mais sensivel a variações em valores preliminares para a matriz $G$ do que para a matriz $R$. Estas duas matrizes apresentam número de condição similar $(2,71 \times 2,40$, respectivamente).

Os componentes de covariância estimados pelo sistema MTC não são os mesmos obtidos pelo método SHC (VALENTE (1988)) e pelo sistema MTDFREML. Aqueles valores correspondem apenas a uma aproximação do máximo da função de verossimilhança restrita, quando adotados como valores preliminares no sistema MTDFREML $\left(-2 \log \left(\mathrm{L}_{2}\right) 590,6582\right.$ para o MTDFREML x 590,7400 para o MTC). As pequenas diferenças observadas resultam em alterações mínimas, não significativas, nas estimativas dos parâmetros genéticos e fenotípicos. A não ser tamanho de amostra muito pequena, não se tem outras explicações que justifiquem as diferenças encontradas, uma vez que houve diagonalização perfeita das matrizes $G$ e $R$ e não há animais endogâmicos nesse conjunto de dados, fatores que poderiam explicar possiveis deferenças em valores estimados pelos programas.

A tabela 12 mostra os resultados obtidos pela análise dos dados do arquivo D411R2 em um modelo de reprodutor. Nesta análise são considerados 3000 registros por característica e um total de 200 equações e quatro características. Os valores iniciais utilizados sãoobtidos por análise univariada prévia, para as variâncias, e para as covariâncias adotam-se valores próximos aos estimados por VALENTE (1988). Como para o arquivo D221, não é possivel avaliar os dados utilizando-se o sistema DFREML.

Os valores estimados, adotando-se os sistemas MTC e MTDFREML, senão idênticos, são muito próximos aos estimados por VALENTE(1988), adotando-se o mesmo método REML e o algoritmo SHC. Os resultados mostram que para amostra de maior tamanho, mantidas as demais condições, os procedimentos convergem para os mesmos valores dos componentes de (co)variância e, conseqüientemente, para o mesmo valor da função de verossimilhança.

Grandes diferenças entre os sistemas são encontradas no tempo e no número de iterações. O sistema MTC convergiu 51 vezes mais rápido e com número de iterações 80 vezes menor. Essas diferenças, além das já apresentados, devem-se especialmente a dois 
fatores. Em primeiro lugar trata-se de uma análise de um sistema não esparso onde a esparsividade (\% de elementos não nulos nas equações do modelo misto (MME)) é de $99,5 \%$. Para situações como essa, os sistemas que adotam o método livre de derivadas, especialmente quando utiliza-se o algoritmo simplex, pelo processo de procura direta sequencial ou linear, mostra-se pouco eficiente, comparado ao EM-REML. Além disso, trata-se de uma análise com quatro características. MISZTAL (1990) e BOLDMAN et al. (1993) citam que análise multivariada com mais de três características devem ser evitadas quando se adota o método DF, uma vez que o processo de estimação pode tornar-se extremamente lento, por requerer número excessivo de avaliações da verossimilhança. Entretanto, embora lento, o método estima adequadamente aos parâmetros e mostra-se bastante útil e preciso na estimação de componentes de covariância, naturalmente requerendo otimizações.

As tabelas de 13 a 13f. mostram os resultados da análise bivariada dos dados do arquivo MUEX2M.D de MEYER (1991). Na tabela 13 apresentam-se as informações gerais referentes ao arquivo cujos dados são analisados. Procura-se realizar uma avaliação pormenorizada dos dados desse arquivo na estimação de componentes de covariancia. Adotam-se diversas estratégias de análise ou formas de fornecimento dos valores iniciais (Tabela 13a), para observar o comportamento de cada um dos sistemas. Além disso, utilizam-se os programas DFMUV e DFMUW do sistema DFREML para verificar a performance relativa de cada um deles nesse tipo de análise.

Alguns parâmetros genéticos calculados a partir dos componentes de covariância estimados pelo sistema MTC são diferentes daqueles obtidos nos demais programas, inclusive os estimados por MEYER (1991). Tais estimativas não resultam no máximo da função de verossimilhança restrita, mas apenas na aproximação dele. Adotando-se as estimativas obtidas pelo programa MTC como valores iniciais nos programas DFMUV e MTDFREML com uma única iteração, foram encontrados, respectivamente, valores de $-2 \log \left(L_{2}\right) \quad 0,08$ e $0,03 \%$ maiores do que aqueles valores encontrados à convergência pelos mesmos programas, adotando-se os valores iniciais mostrados na tabela de resultados.

Esse resultado está relacionado provavelmente ao fato de que, no processo de estimação dos componentes de covariância, utilizando-se a transformação canônica, admite-se diagonalização perfeita da matriz dos efeitos aleatórios $(G)$ inclurdos no modelo, exceto o erro. Todavia, quando há mais de um fator aleatório no modelo, caso desta análise, a diagonalização nem sempre é perfeita. VALENTE (1988), na análise dos dados do arquivo D411R2, também mostra diferenças nas estimativas obtidas por essa técnica e outros programas que utilizam o algoŕt mo EM, sem transformação canônica. Desta forma, verifica-se que essa técnica, embora apresente a grande vantagem quanto à velocidade de convergência, estima componentes de covariância que, em alguns casos, correspondem apenas a uma aproximação do máximo da função de verossimilhança.

Por outro lado, as estimativas das matrizes $G, P$ e $R$ obtidas pelos programas DFMUV e DFMUW, utilizando-se o método simplex ou de Powell e MTDFREML, são praticamente idênticos entre si e aos valores encontradas por MEYER (1991).

Diferentes valores preliminares resultam, em alguns casos, na alteração nos valores estimados para o programa MTC (Tabelas 13b. e 13c.). Essas alterações são da ordem de até $15 \%$ para algum componente de covariancia. Parece, entretanto, que tais variações estão dentro do espaço paramétrico permissıvel e deve-se basicamente à própria 
variação dos dados, devido especialmente ao uso de uma amostra de tamanho reduzido. Os dados ora analisados foram simulados considerando-se efeito aleatório adicional correlacionado com animal (efeito materno). Para os sistemas que utilizam o método livre de derivadas, isso não causa problemas operacionais. No entanto, analisando-se o efeito, desprezando-se correlações existentes, obtém-se $-2 \log \left(\mathrm{L}_{2}\right)$ maior do que seria obtido se aquele efeito fosse considerado. Todavia, o sistema MTC não está preparado para realizar análise em que se considera efeito correlacionado com animal. Esta pode ser uma das explicações para as divergências encontradas.

$\mathrm{Na}$ maioria dos casos, as estimativas nos programas que utilizam o método DF são invariantes a alterações em valores iniciais (pequenas alterações não modificam $-2 \log \left(\mathrm{L}_{2}\right)$ para o nfvel de precisão adotado). No entanto, como já obtido anteriormente, valores distantes das estimativas resultam em aumento substancial no tempo, número de iterações e de avaliações da verossimilhança e às vezes repetições ou tentativas no processo, até obter convergência. O processo de estimação mostra-se muito mais sensivel a valores distantes das estimativas finais para a matriz $G$ e em segundo lugar para a matriz $R$. Esse resultado confirma aqueles obtidos por BOLDMAN et al. (1993).

Adotando-se valores iniciais 10 vezes aos valores sugeridos por MEYER (1991), para a matriz $G$, o sistema DFREML utilizando-se o método simplex não converge utilizando-se "step-size" de $20 \%$, mas converge com "step-size" de $40 \%$ na $1^{\text {a }}$ e $2^{\text {a }}$ tentativas. Mesmo com esse "step-size" e outros mais relaxados, o método de Powell diverge, pois o sistema se interrompe por ocorrência de matriz de efeitos residuais positiva não definida. Assim também, quando se adotam valores preliminares 0,1 vezes aos sugeridos por MEYER (1991), o sistema DFREML, utilizando-se o método simplex, converge apenas com "step-size" de $50 \%$ na sétima tentativa. O método de Powell nesse caso diverge, independente de "step-size's".

Nesta análise, o critério que leva a uma melhor performance do processo de estimação é a estratégia $\mathbf{B}$. Esta estratégia é similar à recomendada por BOLDMAN et al. (1993) e deve ser adotada principalmente quando não se tem uma boa aproximação inicial das estimativas. Em nosso caso, mesmo conhecendo valores preliminares ideais, tal estratégia mostra-se eficiente. Assim, esta fica confirmada como adequada para um processo de estimação adotando-se o metodo livre de derivadas, em análise multivariada.

$\mathrm{O}$ método de Powell apresenta problemas de convergência para valores preliminares 10 vezes aos valores iniciais padrão, $10^{*} G$ e $10^{*} R$, nos programas DFMUV e DFMUW. Adotando-se valores iniciais 10 vezes aos valores padrão (ordem 2 da tabela 13a), não se observa convergência para o máximo absoluto, utilizando-se esses programas, com "step-size" de $20 \%$. Todavia, quando o processo é repetido, adotando-se "step-size" de $40 \%$, nas 2 (duas) primeiras tentativas, o sistema converge no método simplex. Esse resultado é esperado. MEYER (1993) recomenda maiores "step-size's" nas primeiras tentativas, quando os valores preliminares utilizados estão longe das estimativas.

Nota-se claramente que o método de Powell tende a apresentar mais problemas de convergência prematura ou falsa convergência do que o método simplex. O método não admite o surgimento de matrizes não positivas definidas (PD) durante o processo de procura do minimo de $-2 \log \left(\mathrm{L}_{2}\right)$. Isso ocorre com freqủência adotando-se valores iniciais 
distantes das estimativas. Talvez novas rotinas devam ser implementadas para os programas DFMUV e DFMUW, visando prever o surgimento de tais matrizes e fazer correções apropriadas, embora isso possa levar ao aumento do número de iterações e de avaliações da verossimilhança. MEYER (1993) confirma os problemas de falsa convergência do método de Powell, especialmente quando a convergência ocorre no limite do espaço de parâmetro ou quando se trata de superff́cies muito planas.

Em geral, o programa MTC é mais rápido do que os demais programas e, considerando-se convergência normal, os programas DFMUV e DFMUW convergem mais rapidamente, com menor número de iterações e de avaliações da verossimilhança do que o sistema MTDFREML. O programa DFMUV apresenta-se ligeiramente mais rápido do que o programa DFMUW, resultado esperado, pois que este é mais geral. Além disso, admitindo-se convergencia regular, o método de Powell mostra-se bem mais rápido do que o método simplex.

As diferenças em velocidade encontradas entre os programas DFMUV e DFMUW e MTDFREML podem ser parcialmente explicadas. Embora todos programas adotem a linguagem FORTRAN F77 padrão, nota-se, em primeiro lugar, que o sistema DFREML foi desenvolvido dentro de uma filosofia de modularização. Desta forma, os programas são montados através de pequenas rotinas simplificadas, visando a uma perfeita otimização na programação. Em segundo lugar, verifica-se que em uma mesma análise o número de elementos não nulos das MME, após ordenação e fatoração das equações do modelo linear misto, é bem menor para os programas do sistema DFREML. Isso deve-se ao uso de um algorftmo apropriado (minimum degree) que visa reduzir o número de elementos não nulos, gerados durante o processo de ordenação e fatoração das MME. Esse algorítmo também procura aumentar a velocidade do processo de estimação e redução em requerimentos de memória. Nas análises realizadas, o número de elementos não nulos nas MME's estão na ordem: MTDFREML > DFMUW > DFMUV > DFUNI. Não se dispõe dessas informações no programa MTC, embora se saiba que este também adota o mesmo algorftmo utilizado no sistema DFREML. Finalmente, acredita-se que a incorporação e implementação de todo o conjunto de rotinas da Sparspak no sistema MTDFREML, possam ter contribuído para uma perda em velocidade no processo de estimação. No entanto, observam-se melhorias substancias de eficiência na obtenção de estimativas e também na previsão de surgimento de valores fora do espaço de parâmetro. O sistema MTDFREML jamais interrompeu o processamento, por ocorrência de matrizes não P.D's e, ao encerramento do processo de estimação, sempre forneceu estimativas no espaço de parâmetros.

As estimativas de parâmetros genéticos, fenotípicos e de meio ambiente, pela análise dos dados do arquivo MUEX2M.D, são as mesmas para os programas dos sistemas DFREML, MTDFREML e os estimados por MEYER (1991). Essas estimativas são também similares às estimadas no programa MTC, exceto para a herdabilidade da característica 1 e correlação genética entre as características 1 e 2 . 
Tabela 11. Estimativas dos componentes de covariancia, coeficientes de herdabilidade $\left(\hat{h}^{2}\right)$ e correlações genéticas $\left(\hat{r}_{g}\right)$, fenotípicas e de meio ambiente $\left(\hat{r}_{e}\right)$ para os dados do arquivo D221 utilizando-se os programas MTDFRUN e MTC.

Modelo de reprodutor

\begin{tabular}{|c|c|c|c|c|c|c|c|c|c|c|c|c|}
\hline $\begin{array}{l}\text { Ma- } \\
\text { triz }\end{array}$ & $\begin{array}{l}\text { Parâ- } \\
\text { metro }\end{array}$ & $\begin{array}{l}\text { Valores } \\
\text { Iniciais }\end{array}$ & \multicolumn{5}{|c|}{ MTDFRUN } & \multicolumn{5}{|c|}{ MTC } \\
\hline G & $\begin{array}{l}\sigma_{a_{1}}^{2} \\
\sigma_{a_{12}} \\
\sigma_{a_{2}}^{2}\end{array}$ & $\begin{array}{r}, 878 \\
-, 454 \\
1,447\end{array}$ & \multicolumn{5}{|c|}{$\begin{array}{r}, 878 \\
-, 457 \\
1,448 \\
\end{array}$} & \multicolumn{5}{|c|}{$\begin{array}{c}, 959 \\
-, 478 \\
1,332\end{array}$} \\
\hline $\mathrm{R}$ & $\begin{array}{l}\sigma_{e_{1}}^{2} \\
\sigma_{e_{12}} \\
\sigma_{e_{2}}^{2}\end{array}$ & $\begin{array}{l}3,633 \\
-, 340 \\
8,647\end{array}$ & \multicolumn{5}{|c|}{$\begin{array}{l}3,633 \\
-, 340 \\
8,644\end{array}$} & \multicolumn{5}{|c|}{$\begin{array}{l}3,752 \\
-, 306 \\
8,571\end{array}$} \\
\hline & $\begin{array}{l}h_{1}^{2} \\
r_{g_{12}} \\
h_{2}^{2} \\
r_{p_{12}} \\
r_{e_{12}}\end{array}$ & $\begin{array}{c}0,78 \\
-0,40 \\
0,57 \\
-0,12 \\
-0,06 \\
\end{array}$ & \multicolumn{5}{|c|}{$\begin{array}{r}0,78 \\
-0,40 \\
0,57 \\
-0,12 \\
-0,06\end{array}$} & \multicolumn{5}{|c|}{$\begin{array}{c}0,81 \\
-0,42 \\
0,54 \\
-0,11 \\
-0,05\end{array}$} \\
\hline \multicolumn{13}{|c|}{ Informações Adicionais } \\
\hline \multirow{2}{*}{\multicolumn{3}{|c|}{ Tempo(s) }} & (1) & $(2)$ & $(3)$ & $(4)$ & $(5)$ & (1) & $(2)$ & (3) & (4) & $(5)$ \\
\hline & & & 22 & 305 & 180 & 88 & 170 & 9 & 10 & 10 & 84 & 160 \\
\hline \multicolumn{3}{|c|}{ № de Iterações } & 49 & 840 & 539 & 240 & 412 & 3 & 10 & 10 & 230 & 412 \\
\hline \multirow{3}{*}{\multicolumn{3}{|c|}{$\begin{array}{l}\text { № de Avaliações } \\
\text { Vetores de Par. Inválidos } \\
\text { № de Tentativas }\end{array}$}} & 93 & 1436 & 852 & 392 & 752 & - & - & - & - & - \\
\hline & & & 0 & 31 & 22 & 0 & 3 & - & - & - & - & - \\
\hline & & & 1 & 3 & 2 & 2 & 3 & 1 & 1 & 1 & 1 & 1 \\
\hline \multicolumn{3}{|c|}{$-2 \log \left(\mathrm{L}_{2}\right)$} & \multicolumn{5}{|c|}{590,6582} & \multicolumn{5}{|c|}{590,7400} \\
\hline \multicolumn{3}{|c|}{ № de Registros } & \multicolumn{5}{|c|}{214} & \multicolumn{5}{|c|}{214} \\
\hline \multicolumn{3}{|c|}{$\begin{array}{l}\text { № de Equações } \\
\text { Esnarsividade das mme }(\%)\end{array}$} & \multicolumn{5}{|c|}{40} & \multicolumn{5}{|c|}{40} \\
\hline \multicolumn{3}{|c|}{ Esparsividade das mme(\%) } & \multicolumn{5}{|c|}{50,25} & \multicolumn{5}{|c|}{ - } \\
\hline
\end{tabular}

Tabela 11a. Convenções utilizadas na Tabela 11

\begin{tabular}{|c|l|}
\hline Convenção & Descrição \\
\hline$(1)$ & Ajuste a partir dos valores iniciais especificados. \\
$(2)$ & Ajuste a partir dos valores iniciais *10. \\
$(3)$ & Ajuste a partir dos valores iniciais * $0,1$. \\
$(4)$ & Ajuste a partir dos valores iniciais para $\mathrm{G}$ e 10 ou $0,1 * \mathrm{R}$. \\
$(5)$ & Ajuste a partir dos valores iniciais para $\mathrm{R}$ e 10 ou $0,1 * \mathrm{G}$. \\
$\mathrm{R}$ & É a matriz de covariâncias para o erro. \\
$\mathrm{G}$ & É a matriz de covariâncias para os efeitos genéticos aditivos. \\
\hline
\end{tabular}


Tabela 12. Estimativas dos componentes de covariancia para dados do arquivo D411R2, utilizando-se os programas MTDFRUN e MTC - modelo de reprodutor

\begin{tabular}{|c|c|c|c|c|c|c|c|c|}
\hline \multirow{2}{*}{$\begin{array}{l}\text { Para- } \\
\text { metro }\end{array}$} & \multicolumn{2}{|c|}{ Valores Iniciais } & \multicolumn{2}{|c|}{ MTC } & \multicolumn{2}{|c|}{ MTDFRUN } & \multicolumn{2}{|c|}{$\mathrm{SHC}^{+}$} \\
\hline & $G$ & $\mathrm{R}$ & $\mathrm{G}$ & $\mathbf{R}$ & $\mathrm{G}$ & $\mathbf{R}$ & $\mathrm{G}$ & $\mathbf{R}$ \\
\hline$\sigma_{11}$ & $* 145,60$ & $* 639,96$ & 144,84 & 636,68 & 145,39 & 639,97 & 145,60 & 639,96 \\
\hline$\sigma_{12}$ & 18,00 & $-10,00$ & 19,02 & $-9,55$ & 18,97 & $-9,85$ & 19,18 & $-9,79$ \\
\hline$\sigma_{13}$ & 160,00 & $-35,00$ & 162,95 & $-35,68$ & 162,85 & $-36,10$ & 163,26 & $-36,08$ \\
\hline$\sigma_{14}$ & 300,00 & $-25,00$ & 297,48 & $-23,79$ & 297,98 & $-24,35$ & 298,20 & $-24,26$ \\
\hline$\sigma_{22}$ & *137,94 & *1309,79 & 137,99 & 1308,80 & 138,05 & 1309,88 & 137,94 & 1309,79 \\
\hline$\sigma_{23}$ & $-6,00$ & 60,00 & $-6,34$ & 60,75 & $-6,79$ & 60,88 & $-6,24$ & 60,79 \\
\hline$\sigma_{24}$ & $-90,00$ & $-80,00$ & $-86,82$ & $-81,17$ & $-87,35$ & $-81,95$ & $-86,42$ & $-81,85$ \\
\hline$\sigma_{33}$ & $* 498,55$ & ${ }^{*} 2649,99$ & 498,72 & 2650,36 & 497,54 & 2650,06 & 498,55 & 2649,99 \\
\hline$\sigma_{34}$ & 550,00 & 35,00 & 549,70 & 32,45 & 549,22 & 33,15 & 549,64 & 33,34 \\
\hline$\sigma_{44}$ & $* 1087,65$ & $* 5744,30$ & 1087,58 & 5745,26 & 1088,61 & 5744,37 & 1087,65 & 5744,30 \\
\hline \multicolumn{9}{|c|}{ Informações Adicionais } \\
\hline \multicolumn{3}{|c|}{ Tempo(s) } & \multicolumn{2}{|c|}{25} & \multicolumn{2}{|c|}{1293} & \multicolumn{2}{|c|}{. } \\
\hline \multicolumn{3}{|c|}{ № de Iterações } & \multicolumn{2}{|c|}{5} & \multicolumn{2}{|c|}{401} & \multicolumn{2}{|c|}{-} \\
\hline \multicolumn{3}{|c|}{ № de Avaliações } & \multicolumn{2}{|c|}{-} & \multicolumn{2}{|c|}{694} & \multicolumn{2}{|c|}{ - } \\
\hline \multicolumn{3}{|c|}{ Vetores de Par. Invalidos } & \multicolumn{2}{|c|}{-} & \multicolumn{2}{|c|}{0} & \multicolumn{2}{|c|}{-} \\
\hline \multicolumn{3}{|c|}{ Tentativas } & \multicolumn{2}{|c|}{1} & \multicolumn{2}{|c|}{1} & \multicolumn{2}{|c|}{-} \\
\hline \multicolumn{3}{|c|}{$-2 \log \left(\mathrm{L}_{2}\right)$} & \multicolumn{2}{|c|}{102527,69} & \multicolumn{2}{|c|}{102527,69} & \multicolumn{2}{|c|}{-} \\
\hline \multicolumn{3}{|c|}{ № de Registros } & \multicolumn{2}{|c|}{12000} & \multicolumn{2}{|c|}{12000} & \multicolumn{2}{|c|}{ - } \\
\hline $\mathrm{N}^{\circ} \mathrm{de}$ & quações & & & 0 & & & & \\
\hline Espar & vidade(\%) & das mme & & : & & & & \\
\hline
\end{tabular}

${ }^{+}$- Resultados de VALENTE (1988), método SHC ("Short Cut") ${ }^{*}$ - Valor fixado na $1^{\text {a }}$ tentativa

Tabela 13. Informações gerais referentes às análises dos dados do arquivo MUEX2M.D, utilizando os programas DFMUV, DFMUW, MTC e MTDFRUN

\begin{tabular}{|c|c|c|c|c|}
\hline Informação & DFMUV & DFMUW & MTC & MTDFRUN \\
\hline $\mathrm{N}^{\circ}$ de Registros & 568 & 568 & 568 & 568 \\
\hline № de Efeitos Fixos & 3 & 3 & 3 & 3 \\
\hline № de Classes de Efeitos Fixos & 24 & 24 & 24 & 24 \\
\hline № de Efeitos Aleatórios & 3 & 3 & 3 & 3 \\
\hline № Total de Animais & 339 & 339 & 339 & 339 \\
\hline № de Animais na análise & 329 & 329 & 330 & 339 \\
\hline № de Animais com registros & 284 & 284 & 284 & 284 \\
\hline № Classes de Ef. Alea. Adicional & 84 & 84 & 84 & 84 \\
\hline № de Equações & 766 & 766 & 768 & 786 \\
\hline$N^{\circ}$ de Elementos $\neq 0$ nas $\mathrm{MME}$ & 6999 & 10213 & - & 17676 \\
\hline № de Elementos $\neq 0 \mathrm{em} \mathrm{A}^{-1}$ & 658 & 658 & - & 658 \\
\hline Esparsividade das mme(\%) & 2,41 & 3,51 & - & 5,59 \\
\hline
\end{tabular}


Tabela 13a. Valores iniciais utilizados no processo de estimação dos componentes de covariância do arquivo MUEX2M.D

\begin{tabular}{|r|l|}
\hline Ordem & Valores Iniciais adotados \\
\hline 1 & Valores Próximos a convergencia \\
2 & Valores sugeridos por MEYER $(1991)$ \\
3 & 10 vezes os valores utilizados em 2 \\
4 & 0,1 vezes os valores utilizados em 2 \\
5 & Igual a 3 apenas para a matriz G. P e R como em 2 \\
6 & Igual a 3 apenas para a matriz P. G e R como em 2 \\
7 & Igual a 3 apenas para a matriz R. G e P como em 2 \\
8 & Estratégia B \\
\hline
\end{tabular}

Tabela 13b. Estimativas dos componentes de covariancia, coeficientes de herdabilidade $\left(\hat{h}^{2}\right)$ e correlações genéticas $\left(\hat{r}_{g}\right)$, fenotípicas e de meio ambiente $\left(\hat{r}_{e}\right)$ paraos dados do arquivo MUEX2M.D, utilizando-se os programas MTC, DFMUV, DFMUW e MTDFRUN a partir de valores iniciais próximos à convergência

\begin{tabular}{|c|c|c|c|c|c|c|c|c|c|}
\hline \multirow{2}{*}{$\begin{array}{l}\text { Ma- } \\
\text { triz }\end{array}$} & \multirow{2}{*}{$\begin{array}{l}\text { Para- } \\
\text { metro }\end{array}$} & \multirow{2}{*}{$\begin{array}{l}\text { Valores } \\
\text { Iniciais }\end{array}$} & \multicolumn{2}{|c|}{ DFMUV } & \multicolumn{2}{|c|}{ DFMUW } & \multirow{2}{*}{$\begin{array}{r}\text { MTC } \\
E M\end{array}$} & \multirow{2}{*}{$\begin{array}{r}\text { MTDF } \\
(1) \\
\end{array}$} & \multirow{2}{*}{$\begin{array}{r}(\mathrm{KM}) \\
\mathrm{GE} \\
\end{array}$} \\
\hline & & & $(1)$ & $(2)$ & (1) & $(2)$ & & & \\
\hline \multirow{3}{*}{$\mathrm{G}$} & $\sigma_{a_{11}}^{2}$ & 4,90 & 4,98 & 5,05 & 5,02 & 5,05 & 4,23 & 5,04 & 4,99 \\
\hline & $\sigma_{a_{12}}$ & $-1,00$ & $-0,47$ & $-0,39$ & $-0,48$ & $-0,46$ & $-0,98$ & $-0,48$ & $-0,48$ \\
\hline & $\sigma_{a_{22}}^{2}$ & 6,00 & 6,40 & 6,33 & 6,37 & 6,38 & 7,10 & 6,38 & 6,39 \\
\hline \multirow{3}{*}{$\mathrm{P}$} & $\sigma_{p_{11}}^{2 \mu}$ & 1,50 & 1,51 & 1,51 & 1,51 & 1,52 & 1,63 & 1,51 & 1,52 \\
\hline & $\sigma_{p_{12}}$ & $-1,00$ & $-0,76$ & $-0,77$ & $-0,76$ & $-0,77$ & $-0,65$ & $-0,76$ & $-0,75$ \\
\hline & $\sigma_{v_{22}}^{2}$ & 3,00 & 3,02 & 3,03 & 3,03 & 3,04 & 2,88 & 3,02 & 3,08 \\
\hline \multirow{3}{*}{$\mathrm{R}$} & $\sigma_{e_{11}}^{2}$ & 1,70 & 1,65 & 1,62 & 1,64 & 1,62 & 2,13 & 1,62 & 1,63 \\
\hline & $\sigma_{e_{12}}$ & 2,00 & 2,76 & 2,72 & 2,77 & 2,76 & 3,06 & 2,77 & 2,76 \\
\hline & $\sigma_{e_{32}}^{2}$ & 12,60 & 12,45 & 12,49 & 12,48 & 12,46 & 12,39 & 12,48 & 12,43 \\
\hline & & 0,61 & 0,62 & 0,61 & 0,62 & 0,53 & 0,62 & 0,61 \\
\hline \multicolumn{3}{|c|}{$h_{2}^{2}$} & 0,29 & 0,29 & 0,29 & 0,29 & 0,32 & 0,29 & 0,29 \\
\hline \multirow{2}{*}{\multicolumn{3}{|c|}{$r_{g_{12}}$}} & $-0,08$ & $-0,07$ & $-0,08$ & $-0,08$ & $-0,18$ & $-0,08$ & $-0,08$ \\
\hline \multirow{2}{*}{\multicolumn{3}{|c|}{$c_{1}^{2}$}} & 0,18 & 0,18 & 0,18 & 0,18 & 0,20 & 0,18 & 0,19 \\
\hline & & & 0,14 & 0,14 & 0,14 & 0,14 & 0,13 & 0,14 & 0,14 \\
\hline \multicolumn{10}{|c|}{ Informaçōes Adicionais } \\
\hline \multicolumn{3}{|c|}{ Tempo(s) } & 772 & 376 & 981 & 459 & 84 & 1039 & - \\
\hline \multicolumn{3}{|c|}{ № de Iterações } & 313 & 9 & 314 & 9 & 35 & 318 & 481 \\
\hline \multicolumn{3}{|c|}{ № de Avaliações } & 551 & 226 & 547 & 225 & - & 494 & 722 \\
\hline \multicolumn{3}{|c|}{ Vet. Par. Inválidos } & 0 & 0 & 0 & 0 & - & 0 & 0 \\
\hline \multicolumn{3}{|c|}{ Tentativas } & & & 3 & 2 & 1 & 3 & 2 \\
\hline \multicolumn{3}{|c|}{$-2 \log \left(L_{2}\right)$} & \multicolumn{2}{|c|}{1831,77} & \multicolumn{2}{|c|}{1831,77} & 1832,21 & 1831,77 & - \\
\hline
\end{tabular}

(KM) - Resultado de MEYER (1991) MTDF - Programa MTDFRUN (1) - Método Simplex

EM - Maximização de Esperanças GE - Eliminação de Gauss (2) - Método de Powell 
Tabela 13c. Estimativas dos componentes de covariância, coeficientes de herdabilidade $\left(\hat{h}^{2}\right)$ e correlações genéticas $\left(\hat{r}_{g}\right)$, fenotípicas e de meio ambiente $\left(\hat{r}_{e}\right)$ para os dados do arquivo MUEX2M.D, utilizando-se os programas MTC, DFMUV, DFMUW e MTDFRUN a partir de valores iniciais sugeridos por MEYER (1991)

\begin{tabular}{|c|c|c|c|c|c|c|c|c|c|}
\hline \multirow{2}{*}{$\begin{array}{l}\text { Ma- } \\
\text { triz }\end{array}$} & \multirow{2}{*}{$\begin{array}{l}\text { Parâ- } \\
\text { metro }\end{array}$} & \multirow{2}{*}{$\begin{array}{l}\text { Valores } \\
\text { Iniciais }\end{array}$} & \multicolumn{2}{|c|}{ DFMUV } & \multicolumn{2}{|c|}{ DFMUW } & \multirow{2}{*}{$\begin{array}{r}\text { MTC } \\
\text { EM } \\
\end{array}$} & \multirow{2}{*}{$\begin{array}{r}\text { MTDF } \\
(1) \\
\end{array}$} & \multirow{2}{*}{$\begin{array}{r}(\mathrm{KM}) \\
\mathrm{GE} \\
\end{array}$} \\
\hline & & & (1) & (2) & (1) & (2) & & & \\
\hline \multirow{3}{*}{ G } & $\sigma_{a_{11}}^{2}$ & 4,90 & 5,06 & 5,04 & 5,06 & 5,04 & 4,05 & 5,04 & 4,99 \\
\hline & $\sigma_{a_{12}}$ & 1,00 & $-0,47$ & $-0,46$ & $-0,47$ & $-0,46$ & $-0,85$ & $-0,48$ & $-0,48$ \\
\hline & $\sigma_{a_{22}}^{2}$ & 6,00 & 6,35 & 6,42 & 6,35 & 6,42 & 7,10 & 6,38 & 6,39 \\
\hline \multirow{3}{*}{$\mathbf{P}$} & $\sigma_{p_{11}}^{2}$ & 1,50 & 1,51 & 1,51 & 1,51 & 1,51 & 1,63 & 1,51 & 1,52 \\
\hline & $\sigma_{p_{12}}$ & 1,00 & $-0,77$ & $-0,77$ & $-0,77$ & $-0,77$ & $-0,65$ & $-0,76$ & $-0,75$ \\
\hline & $\sigma_{p_{22}}^{2}$ & 3,00 & 3,02 & 3,03 & 3,02 & 3,02 & 2,87 & 3,02 & 3,08 \\
\hline \multirow{3}{*}{$\mathrm{R}$} & $\sigma_{e_{11}}^{2}$ & 1,70 & 1,61 & 1,62 & 1,61 & 1,61 & 2,22 & 1,63 & 1,63 \\
\hline & $\sigma_{e_{12}}$ & 1,00 & 2,77 & 2,77 & 2,77 & 2,77 & 2,99 & 2,77 & 2,76 \\
\hline & $\sigma_{e_{22}}^{2}$ & 12,60 & 12,45 & 12,49 & 12,45 & 12,49 & 12,39 & 12,48 & 12,43 \\
\hline & \multirow{2}{*}{\multicolumn{2}{|c|}{$\begin{array}{l}h_{1}^{2} \\
h_{2}^{2}\end{array}$}} & 0,62 & 0,62 & 0,61 & 0,62 & 0,51 & 0,62 & 0,61 \\
\hline & & & 0,29 & 0,29 & 0,29 & 0,29 & 0,32 & 0,29 & 0,29 \\
\hline \multirow{2}{*}{\multicolumn{3}{|c|}{$\begin{array}{l}r_{g_{12}} \\
c_{1}^{2}\end{array}$}} & $-0,08$ & $-0,08$ & $-0,08$ & $-0,08$ & $-0,16$ & $-0,08$ & $-0,08$ \\
\hline & & & 0,18 & 0,18 & 0,18 & 0,18 & 0,21 & 0,18 & 0,19 \\
\hline \multicolumn{3}{|c|}{$c_{2}^{2}$} & 0,14 & 0,14 & 0,14 & 0,14 & 0,14 & 0,14 & 0,14 \\
\hline \multicolumn{10}{|c|}{ Informações Adicionais } \\
\hline \multicolumn{3}{|c|}{ Tempo(s) } & 844 & 376 & 1109 & 603 & 84 & 2515 & - \\
\hline \multicolumn{3}{|c|}{ № de Iterações } & 372 & 9 & 372 & 12 & 35 & 762 & 481 \\
\hline \multicolumn{3}{|c|}{ № de Avaliações } & 603 & 250 & 603 & 317 & - & 1178 & 722 \\
\hline \multicolumn{3}{|c|}{ Vet. Par. Inválidos } & 0 & 0 & 0 & 0 & 0 & - & 0 \\
\hline \multicolumn{3}{|c|}{ Tentativas } & 3 & 3 & 3 & 3 & 1 & 3 & 2 \\
\hline \multicolumn{3}{|c|}{$-2 \log \left(L_{2}\right)$} & \multicolumn{2}{|c|}{1831,77} & \multicolumn{2}{|c|}{1831,77} & 1832,26 & 1831,77 & - \\
\hline
\end{tabular}

(KM) - Resultado de MEYER (1991)

(1) - Método Simplex

MTDF - Programa MTDFRUN

(2) - Método de Powell 
Tabela 13d. Estimativas dos componentes de covariancia, coeficientes de herdabilidade $\left(\hat{h}^{2}\right)$ e correlações genéticas $\left(\hat{\boldsymbol{r}}_{g}\right)$, fenotf́picas e de meio ambiente $\left(\hat{r}_{e}\right)$ para os dados do arquivo MUEX2M.D, utilizando-se os programas MTC, DFMUV, DFMUW e MTDFRUN a partir de valores iniciais 10 vezes aos sugeridos por MEYER (1991)

\begin{tabular}{|c|c|c|c|c|c|c|c|c|c|}
\hline $\begin{array}{l}\text { Ma- } \\
\text { triz }\end{array}$ & $\begin{array}{l}\text { Para- } \\
\text { metro }\end{array}$ & $\begin{array}{l}\text { Valores } \\
\text { Iniciais }\end{array}$ & $\begin{array}{l}\text { DFML } \\
\text { (1) }\end{array}$ & & $\begin{array}{l}\text { DFML } \\
\text { (1) }\end{array}$ & $\mathrm{w}^{+}$ & $\begin{array}{r}\text { MTC } \\
\text { EM }\end{array}$ & $\begin{array}{r}\text { MTDF } \\
(1)\end{array}$ & $\begin{array}{r}(\mathrm{KM}) \\
\mathrm{GE}\end{array}$ \\
\hline \multirow{3}{*}{$\mathrm{G}$} & $\overline{\sigma_{a_{11}}^{2}}$ & 49 & 5,04 & $+t$ & 5,04 & $+t$ & 4,05 & 5,04 & 4,99 \\
\hline & $\sigma_{a_{12}}$ & 10 & $-0,48$ & $+t$ & $-0,47$ & $+t$ & $-0,86$ & $-0,49$ & $-0,48$ \\
\hline & $\sigma_{a_{22}}^{2}$ & 60 & 6,39 & ++ & 6,39 & $+t$ & 7,10 & 6,39 & 6,39 \\
\hline \multirow{3}{*}{$\mathrm{P}$} & $\sigma_{p_{11}}^{2}$ & 15 & 1,51 & $+t$ & 1,52 & $+t$ & 1,63 & 1,52 & 1,52 \\
\hline & $\sigma_{p_{12}}$ & 10 & $-0,76$ & $+t$ & $-0,76$ & ++ & $-0,65$ & $-0,76$ & $-0,75$ \\
\hline & $\sigma_{p_{22}}^{2}$ & 30 & 3,01 & ++ & 3,01 & ++ & 2,87 & 3,01 & 3,08 \\
\hline \multirow{3}{*}{$\mathrm{R}$} & $\sigma_{e_{11}}^{2}$ & 17 & 1,62 & $+t$ & 1,62 & $+t$ & 2,22 & 1,62 & 1,63 \\
\hline & $\sigma_{e_{12}}$ & 10 & 2,77 & ++ & 2,77 & ++ & 2,99 & 2,78 & 2,76 \\
\hline & $\sigma_{e_{22}}^{2}$ & 126 & 12,45 & ++ & 12,45 & ++ & 12,39 & 12,48 & 12,43 \\
\hline & \multicolumn{2}{|l|}{$h_{1}^{2}$} & 0,62 & $+t$ &, 62 & ++ & 0,51 & 0,62 & 0,61 \\
\hline \multicolumn{3}{|c|}{$h_{2}^{2}$} & 0,29 & $+t$ & 0,29 & $+t$ & 0,32 & 0,29 & 0,29 \\
\hline \multicolumn{3}{|c|}{$r_{g_{12}}$} & $-0,08$ & $+t$ & $-0,08$ & $+t$ & $-0,16$ & $-0,08$ & $-0,08$ \\
\hline \multirow{2}{*}{\multicolumn{3}{|c|}{$\begin{array}{l}c_{1}^{2} \\
c_{2}^{2}\end{array}$}} & 0,18 & ++ & 0,18 & ++ & 0,21 & 0,18 & 0,19 \\
\hline & & & 0,14 & $+t$ & 0,14 & ++ & 0,14 & 0,14 & 0,14 \\
\hline \multicolumn{10}{|c|}{ Informações Adicionais } \\
\hline \multicolumn{3}{|c|}{ Tempo(s) } & 4860 & $+t$ & 5965 & $+t$ & 84 & 4864 & $\cdots$ \\
\hline \multicolumn{3}{|c|}{ № de Iterações } & 1851 & ++ & 1644 & ++ & 35 & 1511 & 481 \\
\hline \multicolumn{3}{|c|}{ № de Avaliações } & 2892 & $+t$ & 2766 & ++ & - & 2511 & 722 \\
\hline \multicolumn{3}{|c|}{ Vet. Par. Inválidos } & 268 & 8302 & 50 & 11044 & - & 132 & 0 \\
\hline \multicolumn{3}{|c|}{ Tentativas } & & & 7 & 3 & 1 & 5 & 2 \\
\hline \multicolumn{3}{|c|}{$-2 \log \left(L_{2}\right)$} & 1831 &, 77 & 183 &, 77 & 1832,27 & 1831,77 & \\
\hline \multicolumn{7}{|c|}{ (KM) - Resultado de MEYER (1991) } & \multicolumn{3}{|c|}{ MTDF - Programa MTDFRUN } \\
\hline
\end{tabular}


Tabela 13e. Estimativas dos componentes de covariancia, coeficientes de herdabilidade $\left(\hat{h}^{2}\right)$ e correlações genéticas $\left(\hat{r}_{g}\right)$, fenotípicas e de meio ambiente $\left(\hat{r}_{e}\right)$ para os dados do arquivo MUEX2M.D, utilizando-se os programas MTC, DFMUV, DFMUW e MTDFRUN. Análise realizada de acordo com a estratégia $\mathbf{B}$

\begin{tabular}{|c|c|c|c|c|c|c|c|c|c|}
\hline \multirow{2}{*}{$\begin{array}{l}\mathrm{Ma} \\
\text { triz }\end{array}$} & \multirow{2}{*}{$\begin{array}{l}\text { Pará- } \\
\text { metro }\end{array}$} & Valores & \multicolumn{2}{|c|}{ DFMUV } & \multicolumn{2}{|c|}{ DFMUW } & \multirow{2}{*}{$\frac{\text { MTC }}{\text { EM }}$} & \multirow{2}{*}{$\frac{\text { MTDF }}{(1)}$} & \multirow{2}{*}{$\frac{(\mathrm{KM})}{\mathrm{GE}}$} \\
\hline & & Iniciais & (1) & (2) & (1) & (2) & & & \\
\hline \multirow{3}{*}{ G } & $\sigma_{a_{11}}^{2}$ & ${ }^{*} 4,90$ & 5,03 & 4,92 & 5,01 & 4,93 & 4,05 & 5,01 & 4,99 \\
\hline & $\sigma_{a_{12}}^{2}$ & 2,00 & $-0,47$ & $-0,45$ & $-0,48$ & $-0,45$ & $-0,85$ & $-0,45$ & $-0,48$ \\
\hline & $\sigma_{a_{22}}^{2}$ & $* 6,00$ & 6,35 & 6,35 & 6,40 & 6,35 & 7,09 & 6,36 & 6,39 \\
\hline \multirow{3}{*}{$P$} & $\sigma_{p_{11}}^{2}$ & $* 1,50$ & 1,51 & 1,52 & 1,51 & 1,51 & 1,63 & 1,51 & 1,52 \\
\hline & $\sigma_{p_{12}}^{2}$ & 2,00 & $-0,77$ & $-0,76$ & $-0,76$ & $-0,75$ & $-0,65$ & $-0,76$ & $-0,75$ \\
\hline & $\sigma_{n_{22}}^{2}$ & $* 3,00$ & 3,02 & 3,03 & 3,00 & 3,03 & 2,87 & 3,02 & 3,08 \\
\hline \multirow{3}{*}{$\mathrm{R}$} & $\sigma_{e_{11}}^{2}$ & ${ }^{*} 1,70$ & 1,63 & 1,68 & 1,64 & 1,68 & 2,22 & 1,64 & 1,63 \\
\hline & $\sigma_{e_{12}}^{2}$ & 2,00 & 2,78 & 2,76 & 2,77 & 2,76 & 2,99 & 2,76 & 2,76 \\
\hline & $\sigma_{e 22}^{2}$ & ${ }^{*} 12,60$ & 12,49 & 12,49 & 12,48 & 12,49 & 12,39 & 12,48 & 12,43 \\
\hline & \multicolumn{2}{|l|}{$h_{1}^{2}$} & 0,62 & 0,62 & 0,61 & 0,62 & 0,51 & 0,62 & 0,61 \\
\hline \multicolumn{3}{|c|}{$h_{2}^{2}$} & 0,29 & 0,29 & 0,29 & 0,29 & 0,32 & 0,29 & 0,29 \\
\hline \multirow{2}{*}{\multicolumn{3}{|c|}{$\begin{array}{l}r_{g_{12}} \\
c_{1}^{2}\end{array}$}} & $-0,08$ & $-0,08$ & $-0,08$ & $-0,08$ & $-0,16$ & $-0,08$ & $-0,09$ \\
\hline & & & 0,18 & 0,18 & 0,18 & 0,18 & 0,21 & 0,18 & 0,19 \\
\hline \multicolumn{3}{|c|}{$c_{2}^{2}$} & 0,14 & 0,14 & 0,14 & 0,14 & 0,14 & 0,13 & 0,14 \\
\hline \multicolumn{10}{|c|}{ Informações Adicionais } \\
\hline \multicolumn{3}{|c|}{ Tempo(s) } & 651 & 327 & 499 & 440 & 80 & 866 & - \\
\hline \multicolumn{3}{|c|}{ № de Iterações } & 183 & 7 & 126 & 7 & 33 & 248 & 481 \\
\hline \multicolumn{3}{|c|}{ № de Avaliações } & 354 & 149 & 240 & 149 & - & 408 & 722 \\
\hline \multicolumn{3}{|c|}{ Vet. Par. Inválidos } & 4 & 1 & 4 & 1 & - & 3 & 0 \\
\hline \multicolumn{3}{|c|}{ Tentativas } & 3 & & 2 & 3 & 1 & 3 & 2 \\
\hline \multicolumn{3}{|c|}{$-2 \log \left(\mathrm{L}_{2}\right)$} & \multicolumn{2}{|c|}{1831,77} & \multicolumn{2}{|c|}{1831,77} & 1832,23 & 1831,77 & - \\
\hline
\end{tabular}

(KM) - Resultado de MEYER (1991) (1) - Método Simplex MTDF - Programa MTDFRUN (2) - Método de Powell

* - Valor fixado na primeira tentativa 
Tabela 13f. Informações adicionais referentes ao desempenho dos programas DFMUV, DFMUW, MTC e MTDFRUN na análise do arquivo MUEX2M.D sob diferentes valores iniciais para as matrizes $G$, $P$ e $R$

\begin{tabular}{|c|c|c|c|c|c|c|}
\hline \multirow[t]{2}{*}{ Descrição } & \multicolumn{2}{|c|}{ DFMUV } & \multicolumn{2}{|c|}{ DFMUW } & \multirow{2}{*}{$\begin{array}{r}\text { MTC } \\
\text { EM }\end{array}$} & \multirow{2}{*}{$\begin{array}{r}\text { MTDF } \\
(1)\end{array}$} \\
\hline & $(1)^{+}$ & $(2)$ & $(1)^{+}$ & $(2)$ & & \\
\hline \multicolumn{7}{|c|}{ Valores Iniciais $0,1^{*}$ Valores de MEYER (1991) } \\
\hline Tempo(s) & 3997 & 650 & 4028 & 665 & 80 & 3625 \\
\hline No de Iterações & 1072 & 18 & 1077 & 17 & 33 & 1154 \\
\hline № de Avaliações & 3075 & 529 & 3082 & 532 & - & 1761 \\
\hline № Vet. com Par. Inválidos & 26 & 18 & 26 & 18 & - & 51 \\
\hline Tentativas & 11 & 3 & 12 & 2 & 1 & 5 \\
\hline \multicolumn{7}{|c|}{ Valores Iniciais $10^{*} \mathrm{G}, \mathrm{P}$ e $\mathrm{R}$ de MEYER (1991) } \\
\hline Tempo(s) & 4478 & 383 & 4979 & $+t$ & 291 & 4877 \\
\hline$N^{\circ}$ de Iterações & 1727 & 9 & 1441 & ++ & 121 & 1219 \\
\hline № de Avaliações & 2954 & 311 & 2385 & ++ & - & 2007 \\
\hline № Vet. com Par. Inválidos & 26 & 11095 & 26 & 11095 & - & 54 \\
\hline Tentativas & 6 & 3 & 5 & 1 & 1 & 6 \\
\hline \multicolumn{7}{|c|}{ Valores Iniciais G, $10^{*} \mathrm{P}$ e R de MEYER (1991) } \\
\hline Tempo(s) & 3058 & 608 & 3327 & 671 & 87 & 2219 \\
\hline № de Iterações & 1350 & 17 & 1356 & 17 & 35 & 701 \\
\hline № de Avaliações & 2091 & 470 & 2176 & 474 & - & 1053 \\
\hline № Vet. com Par. Inválidos & 66 & 18 & 6 & 18 & - & 10 \\
\hline Tentativas & 3 & 3 & 3 & 3 & 1 & 3 \\
\hline \multicolumn{7}{|c|}{ Valores Iniciais G, P e $10^{*} \mathrm{R}$ de MEYER (1991) } \\
\hline Tempo(s) & 6371 & ++ & 6714 & $+t$ & 120 & 2794 \\
\hline № de Iterações & 1428 & ++ & 1474 & $+t$ & 84 & 870 \\
\hline № de Avaliações & 2620 & ++ & 2676 & $+t$ & - & 1340 \\
\hline № Vet. com Par. Inválidos & 41 & ++ & 41 & ++ & - & 28 \\
\hline Tentativas & 7 & - & 7 & - & 1 & 3 \\
\hline \multicolumn{7}{|c|}{ Estratégia $\mathbf{B}$} \\
\hline Tempo(s) & 651 & 327 & 499 & 440 & 80 & 866 \\
\hline № de Iterações & 183 & 7 & 126 & 7 & 33 & 248 \\
\hline № de Avaliações & 354 & 149 & 240 & 149 & 1 & 408 \\
\hline № Vet. com Par. Inválidos & 4 & 1 & 4 & 1 & - & 3 \\
\hline Tentativas & 3 & 3 & 2 & 3 & 1 & 3 \\
\hline
\end{tabular}

(1) - Método Simplex MTDF - Programa MTDFRUN

++ - Matriz de coeficientes não P.D $\quad+$ - "Step-size" de $50 \%$ na $7^{\mathrm{a}}$ tentativa

Os resultados das análises dos arquivos SIMUL050.BEM, SIMUL050.MIS, SIMUL050.PER e SIMUL050.SEL encontram-se nas tabelas de 14 a $14 \mathrm{e}$.

Nesta análise, observando-se as similaridades já constatadas entre os programas DFMUV e DFMUW do sistema DFREML, não se utiliza o programa DFMUW, mas somente o programa DFMUV,

Para o arquivo SIMUL050.BEM, os valores estimados dos componentes de 
covariância, bem como dos parâmetros genéticos, fenotípicos e de meio ambiente são praticamente identicos entre os programas. As diferenças encontradas são para tempo gasto no processo de estimação, número total de iterações e número de avaliações da verossimilhança. Em geral, estes são, em ordem, menores para os sistemas MTC, DFREML pelo método de Powell (DFREML-P), DFREML pelo método simplex (DFREML-S) e MTDFREML. O programa MTC, que utiliza o procedimento EM-REML, é mais rápido do que os programas que utilizam procedimentos livres de derivadas. Nesta análise, o DFREM L-P apresenta convergencia rápida, com poucas avaliações da verossimilhança. Além disso, em todos procedimentos não são observados vetores de parâmetros inválidos, indicando bom número de condição das matrizes, ou seja, as matrizes de componentes de covariância apresentam número de condição tal que não dificulta em demasia o processo de estimação. Além disso, os valores iniciais adotados foram adequados ao processo de estimação.

Verificam-se também diferenças das estimativas dos componentes de variância, para a mesma amostra, utilizando-se análise uni ou multivariada. Esse resultado é confirmado na literatura, como por exemplo no trabalho de LIN (1987) e MEYER (1983). Desta forma, se a seleção de animais for baseada em caracteristicas múltiplas, recomendase adotar parâmetros estimados em análise multivariada. Caso contrário, deve-se adotar parametros estimados em análise univariada.

Os resultados das análises dos dados do arquivo SIMUL050.MIS, com perda de registros, revelam diferenças importantes entre os programas. Para os programas DFREML-S, DFREML-P e MTDFREML, a perda de dado não causa problema operacional ao processo de estimação, mas para o programa MTC a perda de um dado implica na perda total do registro. Desta forma, dependendo do tamanho e distribuição dos dados na amostra, a perda de registro pode causar alterações significativas de valores estimados pelos sistemas que adotam procedimento DF com o EM-REML, com transformação canônica. Para a presente análise, verifica-se igualdade, em termos de valores estimados, entre os sistemas DFREML e MTDFREML. Novamente o método de Powell mostra-se mais eficiente do que o método simplex, por ter gasto menor tempo e número inferior de avaliações da verossimilhança em relação ao DFREML-S e MTDFREML. Nesse procedimento, a perda de dado não traz complicações computacionais, pois no processo de estimação gasta-se, em geral, menor tempo, número de iterações e de avaliações da verossimilhança para se estimar os parâmetros, após a perda de dados.

Os resultados da análise dos dados do arquivo SIMUL050.MIS revelam que a perda de dado causa diminuição no valor da correlação genética entre as caracterusticas 1 e 3 e aumento de correlação de meio ambiente entre as caracteristicas 1 e 2 e 1 e 3 , adotando-se os procedimentos DF, o mesmo não se observa para o programa MTC. Há ligeira elevação dos valores estimados da correlação de meio ambiente entre as caracteristicas 2 e 3 para todos programas.

A partir dos resultados, verifica-se que a perda de dados, mesmo aleatória, pode provocar alterações na estrutura de correlações entre as variáveis, modificando os valores das estimativas dos parâmetros em estudo. Os programas que utilizaram todas informações disponfveis conseguem detectar tais alterações. Assim, dependendo do tipo de experimento, onde a perda de dado para alguma variável for inevitável, de acordo com o percentual de valores perdidos, fica inviável ou pouco recomendável o uso de análise 
multivariada para programas que exigem a perda total do registro.

A análise dos arquivos SIMUL050.PER e SIMUL050.SEL, que são arquivos com dados balanceados, gerados a partir da perda simulada de dados do arquivo SIMUL050.BEM (Tabelas 14c e 14d), revela novamente igualdade entre os sistemas, para modelos que incluem um único fator aleatório além do erro e em dados balanceados. Além disso, confirma a maior lentidão do sistema MTDFREML e maior velocidade de convergência do sistema MTC no processo de estimação dos componentes de covariância. A perda de dados no presente caso, pela eliminação de animais cuja produção no parto anterior foi inferior a 3700 unidades, mostra alteração significativa na estrutura de covariancia entre as características.

Analisando os resultados apresentados nas tabelas $14 \mathrm{~b}$ e $14 \mathrm{e}$, referentes aos valores estimados dos parâmetros genéticos e fenotf́picos, conclui-se que a perda de dados, aleatória ou direcionada, provoca alterações na estrutura de correlação entre as variáveis.

Tabela 14. Estimativas dos componentes de covariancia para os dados do arquivo SIMUL050.BEM, utilizando-se os programas MTC, DFMUV e MTDFRUN. Análise realizada de acordo com a estratégia B - Sem Valores Perdidos

\begin{tabular}{|c|c|c|c|c|c|c|c|}
\hline $\begin{array}{l}\mathrm{Ma-} \\
\text { triz }\end{array}$ & $\begin{array}{l}\text { Para- } \\
\text { metro }\end{array}$ & $\begin{array}{l}\text { Valores dos } \\
\text { parâmetros }\end{array}$ & $\begin{array}{l}\text { Valores } \\
\text { Iniciais }\end{array}$ & $\begin{array}{l}\text { DFM } \\
\text { (1) }\end{array}$ & (2) & $\begin{array}{r}\text { MTC } \\
\text { EM }\end{array}$ & $\begin{array}{r}\text { MTDF } \\
\text { (1) }\end{array}$ \\
\hline \multirow{6}{*}{ G } & $\sigma_{a_{1}}^{2}$ & 173000 & *172630 & 183855 & 184093 & 183516 & 183942 \\
\hline & $\sigma_{a_{12}}$ & 800 & 800 & 754 & 767 & 884 & 762 \\
\hline & $\sigma_{a_{13}}$ & 2200 & 2200 & 2292 & 2290 & 2356 & 2287 \\
\hline & $\sigma_{a_{2}}^{2}$ & 300 & $* 275$ & 349 & 348 & 339 & 349 \\
\hline & $\sigma_{a_{23}}$ & -30 & -30 & -17 & -17 & -21 & -17 \\
\hline & $\sigma_{a_{3}}^{2}$ & 190 & $* 161$ & 183 & 183 & 180 & 183 \\
\hline \multirow{6}{*}{$\mathrm{R}$} & $\sigma_{e_{1}}^{2}$ & 415000 & $* 414271$ & 410520 & 410460 & 411067 & 410606 \\
\hline & $\sigma_{e_{12}}$ & -3700 & -3700 & -3695 & -3705 & -3761 & -3696 \\
\hline & $\sigma_{e_{13}}$ & -3500 & -3500 & -3440 & -3444 & -3500 & -3439 \\
\hline & $\sigma_{e_{2}}^{2}$ & 508 & *588 & 561 & 561 & 568 & 560 \\
\hline & $\sigma_{e_{23}}$ & 246 & 246 & 244 & 244 & 247 & 244 \\
\hline & $\sigma_{e_{3}}^{2}$ & 332 & *332 & 324 & 324 & 325 & 323 \\
\hline \multicolumn{8}{|c|}{ Informações Adicionais } \\
\hline & 1692 & 336 & 47 & 2816 \\
\hline \multicolumn{4}{|c|}{ № de Iterações } & 845 & 9 & 11 & 559 \\
\hline \multicolumn{4}{|c|}{ № de Avaliações } & 1311 & 301 & - & 932 \\
\hline \multicolumn{4}{|c|}{ Vetores de Parametros Inválidos } & 0 & 0 & - & 0 \\
\hline \multicolumn{4}{|c|}{ Tentativas } & 4 & 2 & 1 & 4 \\
\hline \multicolumn{4}{|c|}{$-2 \log \left(\mathrm{L}_{2}\right)$} & 7615,00 & 7615,00 & 7615,00 & 7615,00 \\
\hline \multicolumn{4}{|c|}{ № de Registros } & 903 & 903 & 903 & 903 \\
\hline \multicolumn{4}{|c|}{ № Equações } & 687 & 687 & 678 & 687 \\
\hline \multicolumn{4}{|c|}{ Esparsividade das MME (\%) } & 2,15 & 2,15 & - & 9,27 \\
\hline
\end{tabular}

(1) - Método Simplex MTDF - Programa MTDFRUN (2) - Método de Powell * - Variancias fixadas ao caso univariado na $1^{\text {a }}$ tentativa 
Tabela 14a. Estimativas dos componentes de covariância para os dados do arquivo SIMUL050.MIS, utilizando-se os programas MTC, DFMUV e MTDFRUN. Analise realizada de acordo com a estratégia $\mathbf{B}-\mathbf{2 0} \%$ de valores perdidos para $\mathrm{y}_{2}$ ou $\mathrm{y}_{3}$

\begin{tabular}{|c|c|c|c|c|c|c|}
\hline $\begin{array}{l}\mathrm{Ma}- \\
\text { triz }\end{array}$ & $\begin{array}{l}\text { Pará- } \\
\text { metro }\end{array}$ & $\begin{array}{l}\text { Valores } \\
\text { Iniciais }\end{array}$ & \multicolumn{2}{|c|}{ DFMUV } & $\begin{array}{r}\text { MTC } \\
\text { EM }\end{array}$ & $\begin{array}{r}\text { MTDFRUN } \\
(1)\end{array}$ \\
\hline \multirow{6}{*}{ G } & $\overline{\sigma_{a_{1}}^{2}}$ & $* 172630$ & 193293 & 193484 & 198501 & 193085 \\
\hline & $\sigma_{a_{12}}$ & 800 & 1591 & 1569 & 1823 & 1566 \\
\hline & $\sigma_{a_{13}}$ & 2200 & 633 & 607 & 2715 & 628 \\
\hline & $\sigma_{a_{2}}^{2}$ & *264 & 315 & 315 & 317 & 316 \\
\hline & $\sigma_{a_{23}}$ & -30 & -21 & -20 & -9 & -20 \\
\hline & $\sigma_{a_{3}}^{2}$ & $* 130$ & 198 & 198 & 190 & 198 \\
\hline \multirow{6}{*}{$\mathbf{R}$} & $\sigma_{e_{1}}^{2}$ & $* 414271$ & 404075 & 403862 & 400221 & 404367 \\
\hline & $\sigma_{e_{12}}$ & -3700 & -2394 & -2369 & -4206 & -2377 \\
\hline & $\sigma_{e_{13}}$ & -3500 & -1963 & -1949 & -3403 & -1964 \\
\hline & $\sigma_{e_{2}}^{2}$ & $* 520$ & 502 & 502 & 597 & 502 \\
\hline & $\sigma_{e_{23}}$ & 246 & 231 & 231 & 264 & 231 \\
\hline & $\sigma_{e_{3}}^{2}$ & ${ }^{*} 338$ & 285 & 285 & 299 & 285 \\
\hline \multicolumn{7}{|c|}{ Informações Adicionais } \\
\hline \multicolumn{3}{|c|}{ Tempo(s) } & 1573 & 1108 & 75 & 2197 \\
\hline \multicolumn{3}{|c|}{ № de Iterações } & 441 & 16 & 41 & 441 \\
\hline \multicolumn{3}{|c|}{ № de Avaliações } & 744 & 468 & - & 864 \\
\hline \multicolumn{3}{|c|}{ Vet. Par. Inválidos } & 0 & 0 & - & 0 \\
\hline \multicolumn{3}{|c|}{ Tentativas } & 4 & 4 & 1 & 4 \\
\hline \multicolumn{3}{|c|}{$-2 \log \left(L_{2}\right)$} & 6616,36 & 6616,36 & - & 6616,36 \\
\hline \multicolumn{3}{|c|}{ № de Registros } & 766 & 766 & 612 & 766 \\
\hline \multicolumn{3}{|c|}{ № de Equações } & 673 & 673 & 600 & 673 \\
\hline \multicolumn{3}{|c|}{ Esparsivid. das MME (\%) } & 6,43 & 6,43 & - & 8,25 \\
\hline
\end{tabular}

(1) - Método Simplex

(2) - Método de Powell

* - Variancias fixadas ao caso univariado na $1^{\text {a }}$ tentativa 
Tabela 14b. Estimativas de herdabilidade $\left(\hat{h}^{2}\right)$, repetibilidade $(\hat{r})$ e correlações genéticas $\left(\hat{r}_{g}\right)$, fenotípicas $\left(\hat{r}_{p}\right)$ e de meio ambiente $\left(\hat{r}_{e}\right)$ para os dados das tabelas 14 e $14 \mathrm{a}$, utilizando-se os programas DFMUV, MTC e MTDFRUN

\begin{tabular}{|l|rrrr|rrrr|}
\hline \multirow{2}{*}{$\begin{array}{l}\text { Para- } \\
\text { metro }\end{array}$} & \multicolumn{4}{|c|}{ Tabela 14 } & \multicolumn{4}{c|}{ Tabela 14a } \\
\cline { 2 - 9 } & \multicolumn{2}{|c|}{ DFMUV } & MTC & MTDF & \multicolumn{2}{|c|}{ DFMUV } & MTC & MTDF \\
\cline { 2 - 9 } & $(1)$ & $(2)$ & EM & $(1)$ & $(1)$ & $(2)$ & EM & $(1)$ \\
\hline$h_{1}^{2}$ & 0,31 & 0,31 & 0,31 & 0,31 & 0,32 & 0,32 & 0,33 & 0,32 \\
$h_{2}^{2}$ & 0,38 & 0,38 & 0,37 & 0,38 & 0,39 & 0,39 & 0,35 & 0,39 \\
$h_{3}^{2}$ & 0,36 & 0,36 & 0,36 & 0,36 & 0,41 & 0,41 & 0,39 & 0,41 \\
\hline$r_{g_{12}}$ & 0,09 & 0,10 & 0,11 & 0,10 & 0,20 & 0,20 & 0,23 & 0,20 \\
$r_{g_{13}}$ & 0,40 & 0,39 & 0,41 & 0,39 & 0,10 & 0,10 & 0,44 & 0,10 \\
$r_{g_{23}}$ & $-0,07$ & $-0,07$ & $-0,07$ & $-0,08$ & $-0,08$ & $-0,08$ & $-0,04$ & $-0,08$ \\
\hline$r_{p_{12}}$ & $-0,13$ & $-0,13$ & $-0,12$ & $-0,13$ & $-0,04$ & $-0,04$ & $-0,10$ & $-0,04$ \\
$r_{p_{13}}$ & $-0,07$ & $-0,07$ & $-0,07$ & $-0,07$ & $-0,08$ & $-0,08$ & $-0,04$ & $-0,08$ \\
$r_{p_{23}}$ & 0,33 & 0,33 & 0,33 & 0,33 & 0,33 & 0,33 & 0,38 & 0,33 \\
\hline$r_{e_{12}}$ & $-0,24$ & $-0,24$ & $-0,24$ & $-0,24$ & $-0,17$ & $-0,17$ & $-0,27$ & $-0,17$ \\
$r_{e_{13}}$ & $-0,30$ & $-0,30$ & $-0,30$ & $-0,30$ & $-0,18$ & $-0,18$ & $-0,31$ & $-0,18$ \\
$r_{e_{23}}$ & 0,57 & $\mathbf{0 , 5 7}$ & $\mathbf{0 , 5 7}$ & $\mathbf{0 , 5 7}$ & $\mathbf{0 , 6 1}$ & 0,61 & $\mathbf{0 , 6 2}$ & $\mathbf{0 , 6 1}$ \\
\hline
\end{tabular}

EM - Maximização de Esperanças

(1) - Método simplex

MTDF - Programa MTDFRUN

(2) - Método de Powell 
Tabela 14c. Estimativas dos componentes de covariancia para os dados do arquivo SIMUL050.PER, utilizando-se os programas MTC, DFMUV e MTDFRUN. Análise realizada de acordo com a estratégia $\mathbf{B}-\mathbf{2 0 \%}$ de valores perdidos para $\mathrm{y}_{2}$ ou $\mathrm{y}_{3}$ - com perda total do registro

\begin{tabular}{|c|c|c|c|c|c|c|}
\hline $\begin{array}{l}\text { Ma- } \\
\text { triz }\end{array}$ & $\begin{array}{l}\text { Parâ- } \\
\text { metro }\end{array}$ & $\begin{array}{l}\text { Valores } \\
\text { Iniciais }\end{array}$ & DFMUV & (2) & $\begin{array}{r}\text { MTC } \\
\text { EM }\end{array}$ & $\begin{array}{r}\text { MTDFRUN } \\
\text { (1) }\end{array}$ \\
\hline \multirow{6}{*}{ G } & $\sigma_{a_{1}}^{2}$ & *169263 & 201047 & 200613 & 198501 & 200610 \\
\hline & $\sigma_{a_{12}}$ & 1786 & 1928 & 1922 & 1823 & 1938 \\
\hline & $\sigma_{a_{13}}$ & 2746 & 2648 & 2627 & 2715 & 2650 \\
\hline & $\sigma_{a_{2}}^{2}$ & $* 275$ & 321 & 322 & 317 & 322 \\
\hline & $\sigma_{a_{23}}$ & -20 & -11 & -11 & -9 & -10 \\
\hline & $\sigma_{a_{3}}^{2}$ & $* 141$ & 190 & 190 & 190 & 190 \\
\hline \multirow{6}{*}{$\mathbf{R}$} & $\sigma_{e_{1}}^{2}$ & $* 417519$ & 398747 & 398786 & 400221 & 399101 \\
\hline & $\sigma_{e_{12}}$ & -4188 & -4292 & -4278 & -4206 & -4285 \\
\hline & $\sigma_{e_{13}}$ & -3422 & -3334 & -3324 & -3403 & -3339 \\
\hline & $\sigma_{e_{2}}^{2}$ & ${ }^{*} 644$ & 594 & 594 & 597 & 593 \\
\hline & $\sigma_{e_{23}}$ & 264 & 264 & 264 & 264 & 263 \\
\hline & $\sigma_{e_{3}}^{2}$ & * 329 & $\quad 298$ & 298 & 299 & 298 \\
\hline \multicolumn{7}{|c|}{ Informações Adicionais } \\
\hline & 796 & 485 & 75 & 2225 \\
\hline \multicolumn{3}{|c|}{ No de Iterações } & 480 & 17 & 41 & 695 \\
\hline \multicolumn{3}{|c|}{ № de Avaliações } & 752 & 455 & - & 1047 \\
\hline \multicolumn{3}{|c|}{ Vet. Par. Inválidos } & 0 & 0 & - & 0 \\
\hline \multicolumn{3}{|c|}{ Tentativas } & 3 & 3 & 1 & 3 \\
\hline \multicolumn{3}{|c|}{$-2 \log \left(\mathrm{L}_{2}\right)$} & 4849,95 & 4849,95 & 4850,27 & 4849,95 \\
\hline \multicolumn{3}{|c|}{ № de Registros } & 612 & 612 & 612 & 612 \\
\hline \multicolumn{3}{|c|}{ № de Equações } & 601 & 601 & 600 & 601 \\
\hline \multicolumn{3}{|c|}{ Esparsiv. das MME (\%) } & 2,05 & 2,05 & & 8,48 \\
\hline
\end{tabular}

(1) - Método Simplex

(2) - Método de Powell

* - Variancias fixadas ao caso univariado na $1^{\text {a }}$ tentativa 
Tabela 14d. Estimativas dos componentes de covariância para os dados do arquivo SIMUL050.SEL, utilizando-se os programas DFMUV, MTC e MTDFRUN. Análise realizada de acordo com a estratégia $B$ - perda total do registro posterior se no $1^{\circ}$ parto $y_{1}<$ 3700 unidades

\begin{tabular}{|c|c|c|c|c|c|c|}
\hline $\begin{array}{l}\text { Ma- } \\
\text { triz }\end{array}$ & $\begin{array}{l}\text { Para- } \\
\text { metro }\end{array}$ & $\begin{array}{l}\text { Valores } \\
\text { Iniciais }\end{array}$ & $\begin{array}{l}\text { DFM } \\
\text { (1) }\end{array}$ & (2) & $\begin{array}{r}\text { MTC } \\
\text { EM }\end{array}$ & $\begin{array}{r}\text { MTDFRUN } \\
(1)\end{array}$ \\
\hline \multirow{6}{*}{ G } & $\overline{\sigma_{a_{1}}^{2}}$ & $* 176209$ & 197747 & 198196 & 196073 & 198638 \\
\hline & $\sigma_{a_{12}}$ & 750 & 2318 & 2317 & 2252 & 2312 \\
\hline & $\sigma_{a_{13}}$ & 2200 & -820 & -776 & -720 & -813 \\
\hline & $\sigma_{a_{2}}^{2}$ & *263 & 297 & 294 & 294 & 295 \\
\hline & $\sigma_{a_{23}}$ & -30 & 24 & 23 & 24 & 24 \\
\hline & $\sigma_{a_{3}}^{2}$ & ${ }^{*} 80$ & 84 & 82 & 79 & 83 \\
\hline \multirow{6}{*}{$\mathbf{R}$} & $\sigma_{e_{1}}^{2}$ & $* 406695$ & 395841 & 395780 & 396880 & 395542 \\
\hline & $\sigma_{e_{12}}$ & -3700 & -2759 & -2767 & -2712 & -2762 \\
\hline & $\sigma_{e_{13}}$ & -3500 & -1660 & -1692 & -1732 & -1665 \\
\hline & $\sigma_{e_{2}}^{2}$ & *602 & 587 & 589 & 591 & 588 \\
\hline & $\sigma_{e_{23}}$ & 246 & 211 & 212 & 211 & 211 \\
\hline & $\sigma_{e_{3}}^{2}$ & *378 & 376 & 377 & 378 & 376 \\
\hline \multicolumn{7}{|c|}{ Informações Adicionais } \\
\hline \multicolumn{3}{|c|}{ Tempo(s) } & 926 & 301 & 84 & 1309 \\
\hline \multicolumn{3}{|c|}{ NNo de Iterações } & 540 & 10 & 41 & 406 \\
\hline \multicolumn{3}{|c|}{ № de Avaliações } & 849 & 245 & - & 651 \\
\hline \multicolumn{3}{|c|}{ Vet. Par. Inválidos } & 0 & 0 & - & 0 \\
\hline \multicolumn{3}{|c|}{ Tentativas } & $\quad 3$ & 3 & 1 & 3 \\
\hline \multicolumn{3}{|c|}{$-2 \log \left(L_{2}\right)$} & 5811,14 & 5811,14 & 5811,14 & 5811,14 \\
\hline \multicolumn{3}{|c|}{ № de Registros } & 714 & 714 & 714 & 714 \\
\hline \multicolumn{3}{|c|}{ № de Equações } & 687 & 687 & 678 & 687 \\
\hline \multicolumn{3}{|c|}{ Esparsiv. das м ме (\%) } & 1,82 & 1,82 & - & 8,22 \\
\hline
\end{tabular}

(1) - Método Simplex

(2) - Método de Powell

* - Variancias fixadas ao caso univariado na $1^{\text {a }}$ tentativa 
Tabela 14e. Estimativas de herdabilidade $\left(\hat{h}^{2}\right)$, repetibilidade $(\hat{r})$ e correlações genéticas $\left(\hat{r}_{g}\right)$, fenotf́picas $\left(\hat{r}_{p}\right)$ e de meio ambiente $\left(\hat{r}_{e}\right)$ para os dados das tabelas $14 \mathrm{c}$ e $14 \mathrm{~d}$, utilizando-se os programas DFMUV, MTC e MTDFRUN

\begin{tabular}{|c|c|c|c|c|c|c|c|c|}
\hline \multirow{3}{*}{$\begin{array}{l}\text { Para- } \\
\text { metro }\end{array}$} & \multicolumn{4}{|c|}{ Tabela 14c } & \multicolumn{4}{|c|}{ Tabela 14d } \\
\hline & \multicolumn{2}{|c|}{ DFMUV } & \multirow{2}{*}{$\frac{\mathrm{MTC}}{\mathrm{EM}}$} & \multirow{2}{*}{$\frac{\text { MTDF }}{(1)}$} & \multicolumn{2}{|c|}{ DFMUV } & \multirow{2}{*}{$\frac{\text { MTC }}{\text { EM }}$} & \multirow{2}{*}{$\frac{\text { MT DF }}{(1)}$} \\
\hline & $(1)$ & $(2)$ & & & (1) & $(2)$ & & \\
\hline$h_{1}^{2}$ & 0,33 & 0,33 & 0,33 & 0,33 & 0,33 & 0,33 & 0,33 & 0,33 \\
\hline$h_{2}^{2}$ & 0,35 & 0,35 & 0,35 & & 34 & 0,33 & 0,33 & 0,33 \\
\hline$h_{3}^{2}$ & 0,39 & 0,39 & 0,39 & 0,39 & 0,18 & 0,18 & 0,17 & 0,18 \\
\hline$r_{g_{12}}$ & 0,24 & 0,24 & 0,23 & 0,24 & 0,30 & 0,30 & 0,30 & 0,30 \\
\hline$r_{g_{13}}$ & 0,43 & 0,43 & 0,44 & 43 & $-0,20$ & $-0,19$ & $-0,18$ & $-0,20$ \\
\hline$r_{g_{23}}$ & $-0,04$ & $-0,04$ & $-0,04$ & $-0,04$ & 0,15 & 0,15 & 0,16 & 0,15 \\
\hline$r_{p_{12}}$ & $-0,10$ & $-0,10$ & $-0,10$ & $-0,10$ & $-0,02$ & $-0,02$ & $-0,02$ & $-0,02$ \\
\hline$r_{p_{13}}$ & $-0,04$ & $-0,04$ & $-0,04$ & $-0,04$ & $-0,15$ & $-0,15$ & $-0,15$ & $-0,15$ \\
\hline$r_{p_{23}}$ & 0,38 & 0,38 & 0,38 & 0,38 & 0,37 & 0,37 & 0,37 & 0,37 \\
\hline$r_{e_{12}}$ & $-0,28$ & $-0,28$ & $-0,27$ & $-0,28$ & $-0,18$ & $-0,18$ & $-0,18$ & $-0,18$ \\
\hline$r_{e_{13}}$ & $-0,31$ & $-0,31$ & $-0,31$ & $-0,31$ & $-0,14$ & $-0,14$ & $-0,14$ & $-0,14$ \\
\hline$r_{e_{23}}$ & 0,63 & 0,63 & 0,62 & 0,63 & 0,45 & 0,45 & 0,45 & 0,45 \\
\hline
\end{tabular}

(1) - Método simplex

(2) - Método de Powell

EM - Maximização de Esperanças

MTDF - Programa MTDFRUN

Nas tabelas de 15 a 15c apresentam-se os resultados das análises do arquivo SIMUL050.MAL. Este arquivo foi gerado utilizando-se a mesma estrutura do arquivo SIMUL050.BEM, mas os dados foram simulados baseando-se em matrizes que refletem maiores coeficientes de covariâncias entre as caracterı́sticas. $\mathrm{O}$ objetivo da análise é tentar fazer um paralelo com a análise do arquivo SIMUL050.BEM, cujos dados foram simulados baseando-se em matrizes melhor condicionadas.

Nota-se que os diferentes sistemas não conseguem estimar os mesmos valores para os parâmetros. Observam-se diferenças entre os valores estimados para os três sistemas. Nota-se também muito tempo, número excessivo de avaliações da verossimilhança, de vetores de parâmetros inválidos e de tentativas para que os programas que utilizam o método livre de derivadas alcancem convergência. O maior problema parece ter sido o excessivo número de vetores de parâmetros inválidos. Aparentemente, o sistema MTC comporta-se de forma similar aos demais casos já analisados, mostrando que o método EM-REML é robusto quanto à estimativas próximas ao limite do espaço paramétrico ou matrizes mau condicionadas. Por outro lado, nota-se o efeito do mau condicionamento das matrizes dos componentes a serem estimados, medido por altas correlações, sobre o processo de estimação, adotando-se o método livre de derivadas. Além disso, como mais um indício desse mau condicionamento, o método de Powell do sistema DFREML apresenta convergência irregular, mesmo após inúmeras tentativas com diferentes valores iniciais.

$\mathrm{Na}$ realidade, são realizadas várias tentativas de estimação dos componentes de covariancia, sob diversos vetores de valores iniciais, e na maioria deles o processo 
converge para máximos locais ou encerram o processo de estimação prematuramente no limite do espaço de parâmetros. Novas tentativas não melhoram os valores das estimativas, ou seja, não reduz $-2 \log \left(\mathrm{L}_{2}\right)$. Esse resultado é confirmado por NELDER \& MEAD (1965), POWELL, (1965), MEYER (1993) e por BOLDMAN et al. (1993), os quais afirmam que o método apresenta a tendência de convergir prematuramente quando a convergência ocorre próximo ao limite do espaço de parâmetros ou quando se trata de superf́cies muito planas.

Como observam-se diferenças razoáveis entre os valores estimados para os programas, as analises foram repetidas, adotando-se como valores iniciais as estimativas obtidas pelos demais programas. Os resultados encontram-se nas tabelas 15a, 15b e 15c. Adotando-se os valores estimados pelos programas MTC e DFMUV no sistema MTDFREML, com uma iteração, encontra-se valor para $-2 \log \left(L_{2}\right)$ igual a 6856,92 para o DFMUV e 6856,54 para o MTC. Dessa forma, nesse caso, o sistema MTC, é aquele que encontra melhor nivel de convergência, sendo os resultados alcançados pelos programas DFMUV e MTDFRUN apenas aproximados. Em geral valores iniciais a partir dos resultados encontrados pelo programa MTC são melhores do que aqueles obtidos pelos outros programas. Além disso, diferentes valores iniciais conduzem a alterações nos valores estimados para todos programas, com valores de $-2 \log \left(\mathrm{L}_{2}\right)$ idênticos ou muito similares, dando a indicação de superfície de verossimilhança muito plana, com várias combinações de parâmetros conduzindo ao máximo da função de verossimilhança, para o nı́vel de precisão adotado.

Comparando-se os resultados das análises dos arquivos SIMUL050.BEM e SIMUL050.MAL, verifica-se que, para situações de altas correlações entre as variáveis, o processo de estimação de componentes de covariância torna-se dificultado, havendo grande possibilidade de se estimar inadequadamente aos parâmetros.

Em situações de alta correlação entre as características, MEYER (1993) sugere a fixação de algumas correlações à unidade e, então, estimam-se os componentes remanescentes. $O$ único sistema que permite tal flexibilidade é o sistema DFREML. Por isto, tal estratégia não foi tentada neste trabalho. 
Tabela 15. Estimativas dos componentes de covariancia para os dados do arquivo SIMUL050.MAL, utilizando-se os programas DFMUV, MTC e MTDFRUN. Análise realizada de acordo com a estratégia B

\begin{tabular}{|c|c|c|c|c|c|c|c|}
\hline \multirow{2}{*}{$\begin{array}{l}\mathrm{Ma}- \\
\text { triz }\end{array}$} & \multirow{2}{*}{$\begin{array}{l}\text { Para- } \\
\text { metro }\end{array}$} & \multirow{2}{*}{$\begin{array}{l}\text { Valor do } \\
\text { Parametro }\end{array}$} & \multirow{2}{*}{$\begin{array}{l}\text { Valor } \\
\text { Inicial }\end{array}$} & \multicolumn{2}{|c|}{ DFMUV } & \multirow{2}{*}{$\begin{array}{r}\text { MTC } \\
\text { EM }\end{array}$} & \multirow{2}{*}{$\begin{array}{r}\text { MTDFRUN } \\
\text { (1) }\end{array}$} \\
\hline & & & & (1) & $(2)^{+f}$ & & \\
\hline \multirow{6}{*}{$\mathrm{G}$} & $\sigma_{a_{1}}^{2}$ & 160000 & *154602 & 169613 & 136825 & 179132 & 157915 \\
\hline & $\sigma_{a_{12}}$ & 5959 & 5959 & 6015 & 6011 & 6287 & 5200 \\
\hline & $\sigma_{a_{13}}$ & 593 & 592 & 554 & 780 & 315 & -227 \\
\hline & $\sigma_{a_{2}}^{2}$ & 307 & *307 & 328 & 408 & 358 & 323 \\
\hline & $\sigma_{a_{23}}$ & 156 & 155 & 193 & 235 & 223 & 231 \\
\hline & $\sigma_{a_{3}}^{2}$ & 220 & $* 248$ & 262 & 285 & 329 & 376 \\
\hline \multirow{6}{*}{$\mathbf{R}$} & $\sigma_{e_{1}}^{2}$ & 352000 & *391868 & 372104 & 1519173 & 378580 & 372909 \\
\hline & $\sigma_{e_{12}}$ & 12351 & 12350 & 13145 & 36144 & 13922 & 13885 \\
\hline & $\sigma_{e_{13}}$ & -2995 & -2994 & -3224 & -5436 & -2155 & -1852 \\
\hline & $\sigma_{e_{2}}^{2}$ & 512 & $* 609$ & 549 & 950 & 583 & 588 \\
\hline & $\sigma_{e_{23}}$ & 46 & 45 & 50 & 3,96 & 56 & 65 \\
\hline & $\sigma_{e_{3}}^{2}$ & 408 & ${ }^{*} 392$ & 435 & 383 & 362 & 357 \\
\hline \multicolumn{8}{|c|}{ Informações Adicionais } \\
\hline & 1395 & 364 & 51 & 9582 \\
\hline \multicolumn{4}{|c|}{ № de Iterações } & 778 & 7 & 13 & 2002 \\
\hline \multicolumn{4}{|c|}{ № de Avaliações } & 1538 & 218 & - & 4874 \\
\hline \multicolumn{4}{|c|}{ № Vet. Par. Inválidos } & 280 & 19288 & - & 1251 \\
\hline \multicolumn{4}{|c|}{ № de Tentativas } & 4 & 3 & 2 & 7 \\
\hline \multicolumn{4}{|c|}{$-2 \log \left(L_{2}\right)$} & 6856,92 & - & 6856,54 & 6857,28 \\
\hline \multicolumn{4}{|c|}{ № de Registros } & 903 & 903 & 903 & 903 \\
\hline \multicolumn{4}{|c|}{ № de Equações } & 687 & 687 & 678 & 687 \\
\hline \multicolumn{4}{|c|}{ Esparsividade das MME (\%) } & 2,15 & 2,15 & - & 9,27 \\
\hline
\end{tabular}

(1) - Método Simplex

(2) - Método de Powell

++ - Convergência prematura 
Tabela 15a. Estimativas dos componentes de covariancia para os dados do arquivo SIMUL050.MAL, utilizando-se os programas DFMUV e MTDFRUN. Valores iniciais iguais aos obtidos no programa MTC

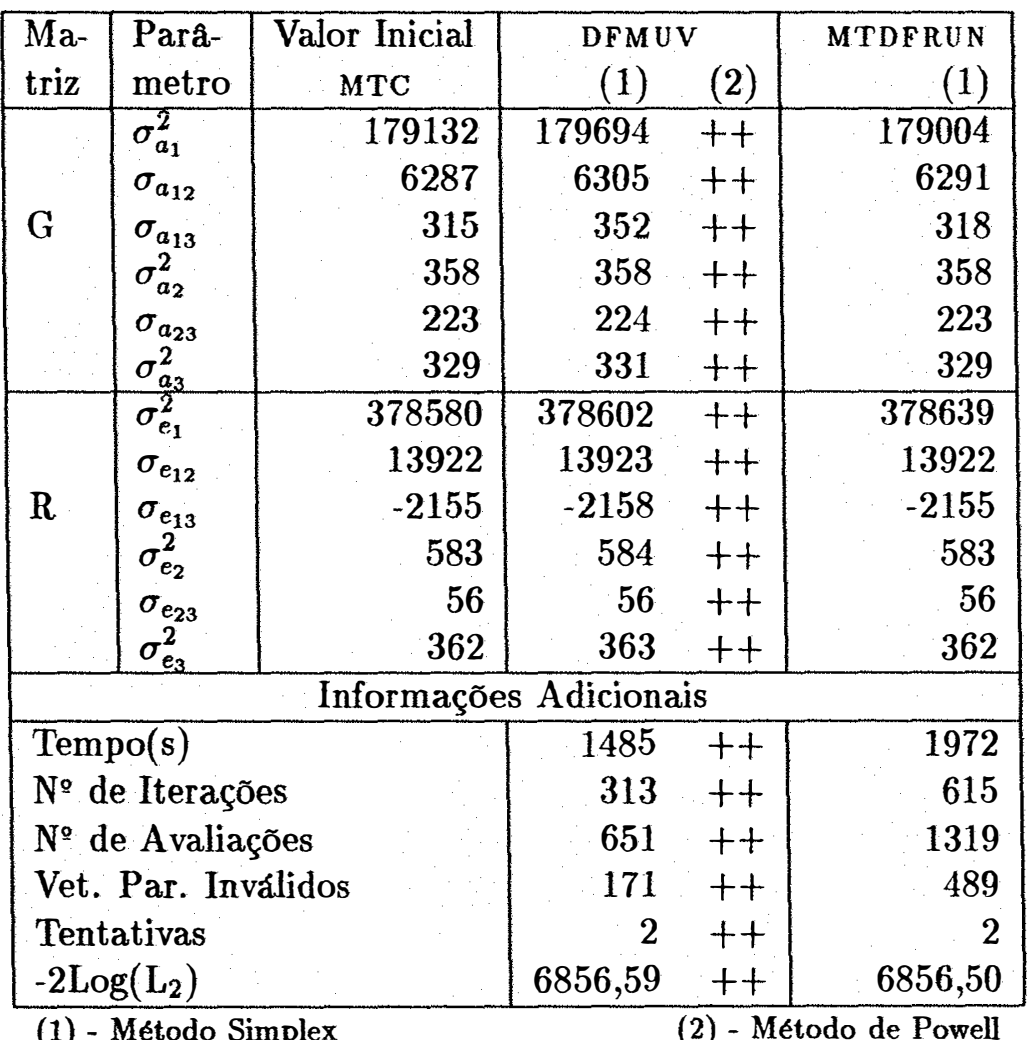

++ - Convergência prematura 
Tabela 15b. Estimativas dos componentes de covariancia para os dados do arquivo SIMUL050.MAL, utilizando-se os programas DFMUV e MTC. Valores iniciais iguais aos obtidos no programa MTDFRUN

\begin{tabular}{|c|c|c|c|c|c|}
\hline $\begin{array}{l}\text { Ma- } \\
\text { triz }\end{array}$ & $\begin{array}{l}\text { Para- } \\
\text { metro }\end{array}$ & $\begin{array}{l}\text { Valor Inicial } \\
\text { MTDFRUN }\end{array}$ & $\begin{array}{l}\text { DFM } \\
\text { (1) }\end{array}$ & (2) & $\begin{array}{r}\text { MTC } \\
\text { EM }\end{array}$ \\
\hline \multirow{6}{*}{ G } & $\sigma_{a_{1}}^{2}$ & 157915 & 160011 & $+t$ & 171716 \\
\hline & $\sigma_{a_{12}}$ & 5200 & 5226 & ++ & 6220 \\
\hline & $\sigma_{a_{13}}$ & -227 & -203 & $+t$ & 751 \\
\hline & $\sigma_{a_{2}}^{2}$ & 323 & 319 & ++ & 361 \\
\hline & $\sigma_{a_{23}}$ & 231 & 230 & $+t$ & 232 \\
\hline & $\sigma_{a_{3}}^{2}$ & 376 & 378 & ++ & 311 \\
\hline \multirow{6}{*}{$\mathrm{R}$} & $\sigma_{e_{1}}^{2}$ & 372909 & 374167 & $+t$ & 383870 \\
\hline & $\sigma_{e_{12}}$ & 13885 & 13896 & $+t$ & 13977 \\
\hline & $\sigma_{e_{13}}$ & -1852 & -1885 & $+t$ & -2453 \\
\hline & $\sigma_{e_{2}}^{2}$ & 588 & 588 & $+t$ & 582 \\
\hline & $\sigma_{e_{23}}$ & 65 & 66 & $+t$ & 51 \\
\hline & $\sigma_{e_{3}}^{2}$ & 357 & 361 & ++ & 376 \\
\hline \multicolumn{6}{|c|}{ Informações Adicionais } \\
\hline \multirow{2}{*}{\multicolumn{3}{|c|}{$\begin{array}{l}\text { Tempo(s) } \\
\text { № de Iteracões }\end{array}$}} & 2517 & $+t$ & 30 \\
\hline \multirow{2}{*}{\multicolumn{3}{|c|}{$\begin{array}{l}\text { № de Iterações } \\
\text { № de Avaliacões }\end{array}$}} & 790 & $+t$ & 14 \\
\hline & & & 1082 & ++ & - \\
\hline \multicolumn{3}{|c|}{ Vet. Par. Inválidos } & 403 & ++ & - \\
\hline \multicolumn{3}{|c|}{ Tentativas } & 3 & ++ & 1 \\
\hline \multicolumn{3}{|c|}{$-2 \log \left(\mathrm{L}_{2}\right)$} & 6857,11 & ++ & 6856,95 \\
\hline
\end{tabular}

(1) - Método Simplex

(2) - Método de Powell

++ - Convergência prematura 
Tabela 15c. Estimativas dos componentes de covariancia para os dados do arquivo SIMUL050.MAL, utilizando-se os programas MTC e MTDFRUN. Valores iniciais iguais aos obtidos no programa DFMUV

\begin{tabular}{|c|c|c|c|c|}
\hline $\begin{array}{l}\mathrm{Ma} \\
\text { triz }\end{array}$ & $\begin{array}{l}\text { Parâ- } \\
\text { metro }\end{array}$ & $\begin{array}{r}\text { Valor Inicial } \\
\text { DFMUV }\end{array}$ & MTC & MTDFRUN \\
\hline G & $\begin{array}{l}\sigma_{a_{1}}^{2} \\
\sigma_{a_{12}} \\
\sigma_{a_{13}} \\
\sigma_{a_{2}}^{2} \\
\sigma_{a_{23}} \\
\sigma_{a_{3}}^{2}\end{array}$ & $\begin{array}{r}169613 \\
6015 \\
554 \\
328 \\
193 \\
262\end{array}$ & $\begin{array}{r}171722 \\
6216 \\
748 \\
361 \\
231 \\
310\end{array}$ & $\begin{array}{r}168212 \\
6015 \\
622 \\
328 \\
193 \\
258\end{array}$ \\
\hline $\mathbf{R}$ & $\begin{array}{l}\sigma_{e_{1}}^{2} \\
\sigma_{e_{12}} \\
\sigma_{e_{13}} \\
\sigma_{e_{2}}^{2} \\
\sigma_{e_{23}} \\
\sigma_{e_{3}}^{2}\end{array}$ & $\begin{array}{r}372104 \\
13145 \\
-3224 \\
549 \\
50 \\
435\end{array}$ & $\begin{array}{r}383928 \\
13978 \\
-2455 \\
582 \\
51 \\
376\end{array}$ & $\begin{array}{r}370060 \\
13155 \\
-3280 \\
548 \\
34 \\
405\end{array}$ \\
\hline \multicolumn{5}{|c|}{ Informações Adicionais } \\
\hline $\begin{array}{l}\text { Tem } \\
\text { № } \\
\text { № } \\
\text { Vet. } \\
\text { Ten } \\
-2 \mathrm{Lc}\end{array}$ & $\begin{array}{l}\text { o(s) } \\
\text { Iteraçó } \\
\text { Avalia } \\
\text { Par. In } \\
\text { tivas } \\
\left(\mathrm{L}_{2}\right)\end{array}$ & $\begin{array}{l}\text { es } \\
\text { idos }\end{array}$ & \begin{tabular}{|r|}
26 \\
8 \\
- \\
- \\
1 \\
6856,94
\end{tabular} & $\begin{array}{r}15485^{*} \\
3047 \\
7964 \\
1578 \\
3 \\
6858,71\end{array}$ \\
\hline
\end{tabular}

* - Convergência diff́cil

As tabelas 16 até $16 \mathrm{~d}$. mostram os valores estimados dos componentes de covariancia para os dados simulados dos arquivos SIMUL400.SEP, SIMUL400.PER, MULTI282.SEP e MULTI615.SEP. Tratam-se de dados sem registros repetidos por animal, e o modelo animal adotado incluiu os efeitos fixos de (HYS), com 354, 353, 2 e 36 classes, respectivamente, e efeito de animal como única fonte de variação aleatória além do erro, com 1800 classes para os dois primeiros conjuntos de dados e 306 classes nos dois últimos. Nos dois primeiros e no último conjunto de dados, as matrizes de covariancias $G$ e $R$, utilizadas para simulação, apresentam número de condição menor do que para os dados simulados para os dados do arquivo MULTI282.SEP.

Os resultados encontrados mostram que os valores estimados adequam-se às estimativas ou valores iniciais adotados. Somente para o arquivo de dados SIMUL400.SEP é que se verificam pequenos desvios entre os valores dos parâmetros e os valores estimados. No entanto, todos os programas estimam valores similares. O método de Powell apresenta convergência irregular para a análise do arquivo SIMUL400.SEP; não é utilizado nas análises dos arquivos SIMUL400.PER e MULTI615.SEP e converge adequadamente nas análises dos dados do arquivo MULTI282.SEP.

A análise do arquivo SIMUL400.PER, onde há perda aleatória simulada 
de $20 \%$ dos registros para as caracteristicas 2 ou 3 , revela que, mesmo para amostras com volume razoável de dados, há alterações nos valores estimados dos componentes de covariância e nas estimativas dos parâmetros genéticos, decorrentes da análise dos dados sem ou com perda total do registro (Tabela $16 \mathrm{~b}$.). Tais alterações provavelmente estão muito mais relacionadas a problemas de amostragem do que realmente diferenças entre programas e métodos. É possivel, no entanto, que a consideração de todos registros disponfveis pelos programas que utilizam o método DF possam contribuir para melhorar a precisão dos valores estimados. Todavia, ainda não se dispõe de formas e fórmulas que permitam calcular os erros padrão das estimativas adotando-se esses métodos.

Note-se que essas análises incluem apenas um efeito aleatório além do erro, ou seja, $G$ inclui apenas as covariâncias dos efeitos genéticos aditivos. Neste caso, normalmente a diagonalização dessa matriz é perfeita, e quando o for, as estimativas obtidas pelos diferentes métodos DF-REML e EM-REML devem ser iguais. Os resultados confirmaram essa tese.

Em geral, verifica-se aumento substancial no tempo, número de iterações e de avaliações da verossimilhança, quanto pior o condicionamento das matrizes envolvidas. $\mathrm{O}$ algoritmo EM-REML confirma a tendência de ser bem mais rápido do que os programas que utilizam o método DF.

Os dados do arquivo MULTI282.SEP, cujas matrizes apresentam condicionamento relativo bom, mostram que todos procedimentos convergem eficientemente bem, utilizando-se os programas DFMUV, DFMUW, MTC e MTDFREML. 
Tabela 16. Estimativas dos componentes de covariancia para os dados do arquivo SIMUL400.SEP, utilizando-se os programas MTC,DFMUV e MTDFRUN. Valores iniciais iguais aos utilizados para simulação -sem perda de dados

\begin{tabular}{|c|c|c|c|c|c|c|}
\hline $\begin{array}{l}\mathrm{Ma-} \\
\text { triz }\end{array}$ & $\begin{array}{l}\text { Pará- } \\
\text { metro }\end{array}$ & $\begin{array}{l}\text { Valores } \\
\text { Iniciais }\end{array}$ & DFMUV & (2) & $\begin{array}{r}\text { MTC } \\
\text { EM }\end{array}$ & $\begin{array}{r}\text { MTDFRUN } \\
\text { (1) }\end{array}$ \\
\hline \multirow{6}{*}{$\mathrm{G}$} & $\overline{\sigma_{a_{1}}^{2}}$ & 173000 & 170178 & $+t$ & 170886 & 169885 \\
\hline & $\sigma_{a_{12}}$ & 2577 & 2992 & ++ & 2961 & 2986 \\
\hline & $\sigma_{a_{13}}$ & 2325 & 1743 & $+t$ & 1824 & 1744 \\
\hline & $\sigma_{a_{2}}^{2}$ & 240 & 259 & ++ & 252 & 259 \\
\hline & $\sigma_{a_{23}}$ & -52 & -54 & ++ & -55 & -55 \\
\hline & $\sigma_{a_{3}}^{2}$ & 125 & 118 & $+t$ & 120 & 119 \\
\hline \multirow{6}{*}{$\mathrm{R}$} & $\sigma_{e_{1}}^{2}$ & 385000 & 384333 & $+t$ & 383869 & 384553 \\
\hline & $\sigma_{e_{12}}$ & -4195 & -3944 & $+t$ & -3934 & -3945 \\
\hline & $\sigma_{e_{13}}$ & -4522 & -3694 & ++ & -3745 & -3697 \\
\hline & $\sigma_{e_{2}}^{2}$ & 508 & 525 & ++ & 530 & 525 \\
\hline & $\sigma_{e_{23}}$ & 246 & 259 & $+t$ & 260 & 260 \\
\hline & $\sigma_{e_{3}}^{2}$ & 332 & 343 & ++ & 342 & 342 \\
\hline \multicolumn{7}{|c|}{ Informações Adicionais } \\
\hline \multirow{2}{*}{\multicolumn{3}{|c|}{ Tempo(s) }} & 5047 & ++ & 366 & 14262 \\
\hline \multirow{2}{*}{\multicolumn{3}{|c|}{$\begin{array}{l}\text { № de Iteraçōes } \\
\text { № de Avaliacões }\end{array}$}} & 515 & $+t$ & 17 & 327 \\
\hline & & & 803 & ++ & - & 500 \\
\hline \multicolumn{3}{|c|}{ Vet. Par. Inválidos } & 0 & - & - & 0 \\
\hline \multicolumn{3}{|c|}{ Tentativas } & 3 & 1 & 1 & 3 \\
\hline \multicolumn{3}{|c|}{$-2 \log \left(\mathrm{L}_{2}\right)$} & 43189,55 & $+t$ & 43189,55 & 43189,55 \\
\hline \multicolumn{3}{|c|}{$N^{\circ}$ de Registros } & 4800 & 4800 & 4800 & 4800 \\
\hline \multicolumn{3}{|c|}{ № de Equações } & 5755 & 5755 & 5685 & 5754 \\
\hline \multicolumn{3}{|c|}{ Esparsiv. das MME (\%) } & 0,31 & 0,31 & - & 0,78 \\
\hline
\end{tabular}

(1) - Método Simplex (2) - Método de Powell t+ - Convergência irregular 
Tabela 16a. Estimativas dos componentes de covariância para os dados do arquivo SIMUL400.PER, utilizando-se os programas MTC, DFMUV e MTDFRUN. Valores iniciais iguais aos utilizados para simulação - perda de $\mathbf{2 0 \%}$ dos dados para $y_{2}$ ou $y_{3}$

\begin{tabular}{|c|c|c|c|c|c|}
\hline $\begin{array}{l}\mathrm{Ma-} \\
\text { triz }\end{array}$ & $\begin{array}{l}\text { Parâ- } \\
\text { metro }\end{array}$ & $\begin{array}{l}\text { Valores } \\
\text { Iniciais }\end{array}$ & $\begin{array}{r}\text { DFMUV } \\
\text { (1) }\end{array}$ & $\begin{array}{r}\mathrm{MTC}^{+} \\
\mathrm{EM}\end{array}$ & $\begin{array}{r}\text { MTDFRUN } \\
\text { (1) }\end{array}$ \\
\hline \multirow{6}{*}{$G$} & $\sigma_{a_{1}}^{2}$ & 173000 & 170992 & 208357 & 170717 \\
\hline & $\sigma_{a_{12}}$ & 2577 & 3006 & 3446 & 2972 \\
\hline & $\sigma_{a_{13}}$ & 2325 & 1974 & 1709 & 1941 \\
\hline & $\sigma_{a_{2}}^{2}$ & 240 & 218 & 243 & 218 \\
\hline & $\sigma_{a_{23}}$ & -52 & -37 & -55 & -39 \\
\hline & $\sigma_{a_{3}}^{2}$ & 125 & 128 & 104 & 128 \\
\hline \multirow{6}{*}{$\mathbf{R}$} & $\sigma_{e_{1}}^{2}$ & 385000 & 384129 & 347967 & 383812 \\
\hline & $\sigma_{e_{12}}$ & -4195 & -3842 & -3948 & -3825 \\
\hline & $\sigma_{e_{13}}$ & -4522 & -3830 & -3646 & -3811 \\
\hline & $\sigma_{e_{2}}^{2}$ & 508 & 531 & 487 & 532 \\
\hline & $\sigma_{e_{23}}$ & 246 & 233 & 246 & 235 \\
\hline & $\sigma_{e_{3}}^{2}$ & 332 & 335 & 370 & 336 \\
\hline \multicolumn{6}{|c|}{ Informações Adicionais } \\
\hline & 22467 & 480 & 28889 \\
\hline \multicolumn{3}{|c|}{ № de Iterações } & 603 & 31 & 720 \\
\hline \multicolumn{3}{|c|}{ № de Avaliações } & 852 & - & 1067 \\
\hline \multicolumn{3}{|c|}{ Vet. Par. Inválidos } & 0 & - & 0 \\
\hline \multicolumn{3}{|c|}{ Tentativas } & 2 & 1 & 2 \\
\hline \multicolumn{3}{|c|}{$-2 \log \left(\mathrm{L}_{2}\right)$} & 36357,24 & - & 36357,24 \\
\hline \multicolumn{3}{|c|}{ № de Registros } & 3853 & 2412 & 3853 \\
\hline \multicolumn{3}{|c|}{ № de Equações } & 5682 & 4140 & 5753 \\
\hline \multicolumn{3}{|c|}{ Esparsiv. das M ME (\%) } & 0,94 & - & 0,726 \\
\hline
\end{tabular}

+ - Todo registro é perdido

(1) - Método Simplex 
Tabela 16b. Estimativas de herdabilidade $\left(\hat{h}^{2}\right)$, repetibilidade $(\hat{r})$ e correlações genéticas $\left(\hat{r}_{g}\right)$, fenotf́picas $\left(\hat{r}_{p}\right)$ e de meio ambiente, $\left(\hat{r}_{e}\right)$ para os dados das tabelas 16 e 16a., utilizando-se os programas DFMUV, MTC e MTDFRUN

\begin{tabular}{|l|rrr|rrr|}
\hline \multirow{2}{*}{$\begin{array}{l}\text { Parå- } \\
\text { metro }\end{array}$} & \multicolumn{3}{|c|}{ Tabela 16 } & \multicolumn{4}{|c|}{ Tabela 16a } \\
\cline { 2 - 7 } & DFMUV & MTC & MTDFRUN & DFMUV & MTC & MTDF RUN \\
\cline { 2 - 7 } & $(1)$ & EM & $(1)$ & $(1)$ & EM & $(1)$ \\
\hline$h_{1}^{2}$ & 0,31 & 0,31 & 0,31 & 0,31 & 0,37 & 0,31 \\
$h_{2}^{2}$ & 0,33 & 0,32 & 0,33 & 0,29 & 0,33 & 0,29 \\
$h_{3}^{2}$ & 0,26 & 0,26 & 0,26 & 0,28 & 0,22 & 0,28 \\
\hline$r_{g_{12}}$ & 0,45 & 0,45 & 0,45 & 0,49 & 0,48 & 0,49 \\
$r_{g_{13}}$ & 0,39 & 0,40 & 0,40 & 0,42 & 0,37 & 0,42 \\
$r_{g_{23}}$ & $-0,31$ & $-0,32$ & $-0,31$ & $-0,22$ & $-0,34$ & $-0,23$ \\
\hline$r_{p_{12}}$ & $-0,05$ & $-0,05$ & $-0,05$ & $-0,04$ & $-0,02$ & $-0,04$ \\
$r_{p_{13}}$ & $-0,12$ & $-0,12$ & $-0,12$ & $-0,12$ & $-0,12$ & $-0,12$ \\
$r_{p_{23}}$ & 0,34 & 0,34 & 0,34 & 0,33 & 0,33 & 0,33 \\
\hline$r_{e_{12}}$ & $-0,28$ & $-0,28$ & $-0,28$ & $-0,27$ & $-0,30$ & $-0,27$ \\
$r_{e_{13}}$ & $-0,32$ & $-0,33$ & $-0,32$ & $-0,34$ & $-0,32$ & $-0,34$ \\
$r_{e_{23}}$ & 0,61 & 0,61 & 0,61 & 0,55 & 0,58 & 0,56 \\
\hline
\end{tabular}


Tabela 16c.Estimativas dos componentes de covariancia, coeficientes de herdabilidade $\left(\hat{h}^{2}\right)$ e correlações genéticas $\left(\hat{r}_{g}\right)$ para os dados do arquivo MULTI282.SEP, utilizando-se os programas DFMUV, DFMUW, MTC e MTDFRUN. Análise realizada de acordo com a estratégia $\mathbf{B}$

\begin{tabular}{|c|c|c|c|c|c|c|c|c|}
\hline \multirow{2}{*}{$\begin{array}{l}\text { Ma- } \\
\text { triz }\end{array}$} & \multirow{2}{*}{$\begin{array}{l}\text { Para- } \\
\text { metro }\end{array}$} & \multirow{2}{*}{$\begin{array}{l}\text { Valores } \\
\text { Iniciais }\end{array}$} & \multicolumn{2}{|c|}{ DFMUV } & \multicolumn{2}{|c|}{ DFMUW } & \multirow{2}{*}{$\begin{array}{r}\text { MTC } \\
\text { EM } \\
\end{array}$} & \multirow{2}{*}{$\frac{\text { MTDFRUN }}{(1)}$} \\
\hline & & & (1) & (2) & (1) & $(2)$ & & \\
\hline \multirow{6}{*}{$\mathbf{G}$} & $\overline{\sigma_{a_{1}}^{2}}$ & *181918 & 182983 & 182864 & 182781 & 182866 & 182642 & 183289 \\
\hline & $\sigma_{a_{12}}$ & 3500 & 3498 & 3494 & 3502 & 3494 & 3402 & 3501 \\
\hline & $\sigma_{a_{13}}$ & 1800 & 1791 & 1806 & 1780 & 1806 & 1909 & 1790 \\
\hline & $\sigma_{a_{2}}^{2}$ & ${ }^{*} 226$ & 228 & 229 & 229 & 229 & 225 & 228 \\
\hline & $\sigma_{a_{23}}$ & 20 & 23 & 24 & 23 & 24 & 12 & 23 \\
\hline & $\sigma_{a_{3}}^{2}$ & $* 165$ & 147 & 148 & 147 & 148 & 143 & 147 \\
\hline \multirow{12}{*}{$\mathbf{R}$} & $\sigma_{e_{1}}^{2}$ & *396084 & 396314 & 396387 & 396172 & 396386 & 395994 & 396035 \\
\hline & $\sigma_{e_{12}}$ & -4195 & -3841 & -3829 & -3849 & -3829 & -3785 & -3848 \\
\hline & $\sigma_{e_{13}}$ & -3522 & -3579 & -3586 & -3576 & -3586 & -3672 & -3581 \\
\hline & $\sigma_{e_{2}}^{2}$ & $* 458$ & 456 & 454 & 455 & 454 & 458 & 456 \\
\hline & $\sigma_{e_{23}}$ & 200 & 193 & 192 & 193 & 192 & 201 & 194 \\
\hline & $\sigma_{e_{3}}^{2}$ & *305 & 315 & 314 & 315 & 314 & 320 & 316 \\
\hline & $h_{1}^{2}$ &, 31 & 0,32 & 0,32 & 0,32 & 0,32 & 0,32 & 0,32 \\
\hline & $r_{g_{12}}$ &, 55 & 0,54 & 0,54 & 0,54 & 0,54 & 0,53 & 0,54 \\
\hline & $r_{g_{13}}$ & ,33 & 0,35 & 0,35 & 0,35 & 0,35 & 0,37 & 0,35 \\
\hline & $h_{2}^{2}$ & ,33 & 0,33 & 0,33 & 0,33 & 0,33 & 0,33 & 0,33 \\
\hline & $h_{3}^{2}$ & ,35 & 0,32 & 0,32 & 0,32 & 0,32 & 0,31 & 0,32 \\
\hline & $r_{g_{23}}$ &, 10 & 0,13 & 0,13 & 0,13 & 0,13 & 0,07 & 0,13 \\
\hline \multicolumn{9}{|c|}{ Informações Adicionais } \\
\hline & 861 & 423 & 1058 & 578 & 37 & 1463 \\
\hline \multicolumn{3}{|c|}{ № de Iterações } & 443 & 14 & 396 & 14 & 21 & 506 \\
\hline \multicolumn{3}{|c|}{ № de Avaliações } & 731 & 361 & 676 & 361 & 1 & 818 \\
\hline \multicolumn{3}{|c|}{ Vet. Par. Inválidos } & 0 & 0 & 0 & 0 & 0 & 0 \\
\hline \multicolumn{3}{|c|}{ Tentativas } & $\begin{array}{l}3 \\
: \quad 3\end{array}$ & 3 & 3 & 3 & 1 & 3 \\
\hline \multicolumn{3}{|c|}{$-2 \log \left(\mathrm{L}_{2}\right)$} & 7980,00 & 7980,00 & 7980,00 & 7980,00 & 7980,12 & 7980,00 \\
\hline \multicolumn{3}{|c|}{ № de Registros } & 846 & 846 & 848 & 846 & 846 & 846 \\
\hline \multicolumn{3}{|c|}{ № de Equações } & 925 & 925 & 925 & 925 & 924 & 925 \\
\hline \multicolumn{3}{|c|}{ № de Animais } & 306 & 306 & 306 & 306 & 306 & 306 \\
\hline \multicolumn{3}{|c|}{ Ef. Aleat. Adicionais } & 400 & 400 & 400 & 400 & 400 & 400 \\
\hline \multicolumn{3}{|c|}{ Class. Ef. Fixos } & 2 & 2 & 2 & 2 & 2 & 2 \\
\hline \multicolumn{3}{|c|}{ Espars.das MME(\%) } & 0,68 & 0,68 & 2,25 & 2,25 & - & 4,26 \\
\hline
\end{tabular}

(1) - Método Simplex ${ }^{*}$ - Variancias fixadas caso univariado $1^{a}$ tentativa (2) - Método de Powell 
Tabela 16d. Estimativas dos componentes de covariancia, coeficientes de herdabilidade $\left(\hat{h}^{2}\right)$ e correlação genética $\left(\hat{r}_{g}\right)$ para os dados do arquivo MULTI615.SEP, utilizando-se os programas DFMUV, MTC e MTDFRUN. Valores iniciais iguais aos utilizados para simulação

\begin{tabular}{|c|c|c|c|c|c|}
\hline \multirow{2}{*}{$\begin{array}{l}\mathrm{Ma}- \\
\text { triz }\end{array}$} & \multirow{2}{*}{$\begin{array}{l}\text { Para- } \\
\text { metro }\end{array}$} & \multirow{2}{*}{$\begin{array}{l}\text { Valores } \\
\text { Iniciais }\end{array}$} & DFMUV & MTC & MTDFRUN \\
\hline & & & (1) & $\mathrm{EM}$ & $(1)$ \\
\hline \multirow{6}{*}{ G } & $\overline{\sigma_{a_{1}}^{2}}$ & 173000 & 176258 & 186717 & 176545 \\
\hline & $\sigma_{a_{12}}$ & 2577 & 3420 & 2614 & 2827 \\
\hline & $\sigma_{a_{13}}$ & 2325 & 2712 & 2710 & 2890 \\
\hline & $\sigma_{a_{2}}^{2}$ & 240 & 246 & 226 & 252 \\
\hline & $\sigma_{a_{23}}$ & -52 & -56 & -43 & -63 \\
\hline & $\sigma_{a_{3}}^{2}$ & 125 & 117 & 126 & 113 \\
\hline \multirow{12}{*}{$\mathrm{R}$} & $\sigma_{e_{1}}^{2}$ & 385000 & 393556 & 388411 & 393474 \\
\hline & $\sigma_{e_{12}}$ & -4195 & -4094 & -3688 & -3807 \\
\hline & $\sigma_{e_{13}}$ & -4522 & -3520 & -3525 & -3608 \\
\hline & $\sigma_{e_{2}}^{2}$ & 508 & 468 & 478 & 465 \\
\hline & $\sigma_{e_{23}}$ & 246 & 237 & 231 & 241 \\
\hline & $\sigma_{e_{3}}^{2}$ & 332 & 311 & 307 & 313 \\
\hline & $h_{1}^{2}$ & 0,31 & 0,31 & 0,32 & 0,31 \\
\hline & $r_{g_{12}}$ & 0,40 & 0,52 & 0,40 & 0,42 \\
\hline & $r_{g_{13}}$ & 0,50 & 0,60 & 0,56 & 0,65 \\
\hline & $h_{2}^{2}$ & 0,32 & 0,34 & 0,32 & 0,35 \\
\hline & $h_{3}^{\tilde{2}}$ & 0,27 & 0,27 & 0,29 & 0,27 \\
\hline & $r_{g_{23}}$ & $-0,30$ & $-0,33$ & $-0,26$ & $-0,37$ \\
\hline \multicolumn{6}{|c|}{ Informações Adicionais } \\
\hline \multicolumn{3}{|c|}{ Tempo(s) } & 310 & 11 & 851 \\
\hline \multicolumn{3}{|c|}{ № de Iterações } & 202 & 6 & 259 \\
\hline \multicolumn{3}{|c|}{ № de Avaliações } & 357 & - & 468 \\
\hline \multicolumn{3}{|c|}{ Vet. Par. Inválidos } & 0 & 0 & 0 \\
\hline \multicolumn{3}{|c|}{ Tentativas } & 2 & 1 & 2 \\
\hline \multicolumn{3}{|c|}{$-2 \log \left(\mathrm{L}_{2}\right)$} & 7139,33 & 7139,33 & 7139,33 \\
\hline \multicolumn{3}{|c|}{ № de Registros } & 846 & 846 & 846 \\
\hline \multicolumn{3}{|c|}{ № de Equações } & 1026 & 1026 & 1026 \\
\hline \multicolumn{3}{|c|}{ № de Animais } & 306 & 306 & 306 \\
\hline \multicolumn{3}{|c|}{ Class. Ef. Fixos } & 36 & 36 & 36 \\
\hline \multicolumn{3}{|c|}{ Espars.das MME(\%) } & 0,68 & - & 3,50 \\
\hline
\end{tabular}

Os resultados das analises do arquivo SIMUL100.ARQ, com e sem valores perdidos, são apresentados nas tabelas de 17 a 17c. Para este arquivo de dados há instabilidade à convergencia para o sistema MTC, ou seja, a continuação do processo de estimação, sugerindo novas iterações após a convergência, às vezes, reverte o processo. Nesta análise, encerrou o processo quando os parâmetros de parada apresentavam os seguintes valores : 
convR $=0,25 \cdot 10^{-7}$, ConvG $=0,27 \cdot 10^{-6}$ e $0,91 \cdot 10^{-6}$, e soma dos elementos for a da diagonal iguais a 0,027 e 0,035 , para as matrizes $G$ e $P$, respectivamente.

Os valores estimados pelos diversos programas são relativamente próximos aos valores dos parametros exceto alguns valores estimados pelo programa MTC. A rigor não se observou convergência para esse programa. Os valores apresentados na tabela são apenas aqueles obtidos próximos à convergência que não correspondem ao máximo da função de verossimilhança restrita (veja $-2 \log \left(\mathrm{L}_{2}\right)$ ). Em geral, para modelos que incluem mais de um efeito aleatório além do erro, a diagonalização da matriz $G$ que inclui os efeitos genéticos aditivos e efeitos permanentes de meio $(G+P)$ não é perfeita. Desta forma, mesmo quando há convergência, os valores estimados pelo método EM-REML, através do programa MTC, são apenas aproximados. Em geral, a aproximação é boa.

Como na simulação adotam-se valores das características baseados em matrizes de valores genéticos aditivos, permanentes de meio e do resíduo, mal condicionadas, há grande dificuldade para conseguir convergência. Isso pode ser constatado, verificandose o tempo, número de iterações e de vetores de paråmetros inválidos, obtidos nos sistemas que utilizam o método livre de derivadas.

Quando se utilizam valores 10 vezes ou 0,1 vezes aos valores utilizados para simulação, ou seja valores iniciais distantes das estimativas (Tabela 17a), a convergência é completamente irregular para os sistemas DFREML e MTDFREML. O sistema MTC manteve o mesmo padrão apresentado para valores iniciais iguais aos utilizados para simulação, ou seja, os valores estimados utilizando-se o método EM-REML apresentam pouca variação quando se fornecem valores iniciais próximos ou distantes dos parámetros. Por outro lado, quando a opção é de se utilizar os métodos livres de derivadas, é necessário que o usuário se preocupe com o fornecimento de valores próximos dos valores das estimativas. Caso não haja conhecimentos de valores iniciais próximos à convergência, o ideal é adotar no processo de estimação a estratégia $B$, para procurar aproximações razoáveis de valores iniciais, que deverão ser adotados e assim aumentar a probabilidade de se obter estimativas que correspondam ao máximo absoluto da função de verossimilhança. Tal estratégia é também importante para reduzir o tempo e número de avaliações da verossimilhança. 
Tabela 17. Estimativas dos componentes de covariancia para os dados do arquivo SIMUL100.ARQ, utilizando-se os programas DFMUV,MTC e MTDFRUN. Valores iniciais iguais aos utilizados para simulação

\begin{tabular}{|c|c|c|c|c|c|}
\hline $\begin{array}{l}\text { Ma- } \\
\text { triz }\end{array}$ & $\begin{array}{l}\text { Parâ- } \\
\text { metro }\end{array}$ & $\begin{array}{l}\text { Valores } \\
\text { Iniciais }\end{array}$ & $\begin{array}{r}\text { DFMUV } \\
(1)\end{array}$ & $\begin{array}{r}\text { MTC } \\
* * \\
\end{array}$ & $\begin{array}{r}\text { MTDFRUN } \\
(1) \\
\end{array}$ \\
\hline \multirow{6}{*}{ G } & $\overline{\sigma_{a_{1}}^{2}}$ & 160000 & 153047 & 164222 & 153406 \\
\hline & $\sigma_{a_{12}}$ & 5959 & 5937 & 6755 & 5898 \\
\hline & $\sigma_{a_{13}}$ & 593 & 564 & -339 & 631 \\
\hline & $\sigma_{a_{2}}^{2}$ & 307 & 310 & 354 & 306 \\
\hline & $\sigma_{a_{23}}$ & 156 & 153 & 126 & 154 \\
\hline & $\sigma_{a_{3}}^{2}$ & 220 & 216 & 167 & $\begin{array}{r}217 \\
\end{array}$ \\
\hline \multirow{6}{*}{$P$} & $\sigma_{p_{1}}^{2}$ & 128000 & 130015 & 134028 & 127127 \\
\hline & $\sigma_{p_{12}}$ & 4730 & 4679 & 4741 & 4560 \\
\hline & $\sigma_{p_{13}}$ & -2078 & -1982 & -1279 & -2257 \\
\hline & $\sigma_{p_{2}}^{2}$ & 205 & 204 & 203 & 195 \\
\hline & $\sigma_{p_{23}}$ & -22 & -17 & 20 & -35 \\
\hline & $\sigma_{p_{3}}^{2}$ & 157 & 139 & $\quad 170$ & 125 \\
\hline \multirow{6}{*}{$\mathbf{R}$} & $\sigma_{e_{1}}^{2}$ & 352000 & 354710 & 350691 & 359583 \\
\hline & $\sigma_{e_{12}}$ & 12351 & 12339 & 11966 & 12431 \\
\hline & $\sigma_{e_{13}}$ & -2995 & -2887 & -3520 & -3004 \\
\hline & $\sigma_{e_{2}}^{2}$ & 512 & 504 & 479 & 502 \\
\hline & $\sigma_{e_{23}}$ & 46 & 50 & 19 & 42 \\
\hline & $\sigma_{e_{3}}^{2}$ & 408 & 421 & 407 & 412 \\
\hline \multicolumn{6}{|c|}{ Informações Adicionais } \\
\hline & 35232 & 1564 & 73983 \\
\hline \multicolumn{3}{|c|}{ № de Iterações } & 2619 & 162 & 4056 \\
\hline \multicolumn{3}{|c|}{ № de Avaliações } & 3967 & 1 & 6715 \\
\hline \multicolumn{3}{|c|}{ Vet. Par. Inválidos } & 421 & - & 967 \\
\hline \multicolumn{3}{|c|}{ Tentativas } & 4 & $\therefore 1$ & 6 \\
\hline \multicolumn{3}{|c|}{$-2 \log \left(\mathrm{L}_{2}\right)$} & 22114,90 & 22118,47 & 22114,96 \\
\hline \multicolumn{3}{|c|}{ № de Registros } & 2742 & 2742 & 2742 \\
\hline \multicolumn{3}{|c|}{ № de Animais } & 1500 & 1410 & 1500 \\
\hline \multicolumn{3}{|c|}{ Clas. Ef. Aleat. Adicionais } & 400 & 400 & 400 \\
\hline \multicolumn{3}{|c|}{ № Class. Ef. Fixos } & 249 & 249 & 249 \\
\hline \multicolumn{3}{|c|}{ № Equações } & 2949 & 2856 & 2949 \\
\hline \multicolumn{3}{|c|}{ Esparsivid. das MME(\%) } & 1,38 & - & 2,42 \\
\hline
\end{tabular}

* - Valores fixados na primeira tentativa 
Tabela 17a. Estimativas dos componentes de covariancia para os dados do arquivo SIMUL100.ARQ, utilizando-se os programas DFMUV, MTC e MTDFRUN. Valores iniciais $\mathbf{0 , 1}$ vezes aos utilizados para simulação

\begin{tabular}{|c|c|c|c|c|c|}
\hline $\begin{array}{l}\text { Ma- } \\
\text { triz }\end{array}$ & $\begin{array}{l}\text { Para- } \\
\text { metro }\end{array}$ & $\begin{array}{l}\text { Valores } \\
\text { Iniciais }\end{array}$ & $\begin{array}{r}\text { DFMUV } \\
(1)\end{array}$ & MTC & $\begin{array}{r}\text { MTDFRUN } \\
(1) \\
\end{array}$ \\
\hline \multirow{6}{*}{$\mathrm{G}$} & $\sigma_{a_{1}}^{2}$ & 16000,0 & 28853 & 164847 & 6780 \\
\hline & $\sigma_{a_{12}}$ & 595,9 & 548 & 6783 & 283 \\
\hline & $\sigma_{a_{13}}$ & 59,3 & 105 & 370 & 68 \\
\hline & $\sigma_{a_{2}}^{2}$ & 30,7 & 83 & 355 & 16 \\
\hline & $\sigma_{a_{23}}$ & 15,6 & 11 & 126 & 1 \\
\hline & $\sigma_{a_{3}}^{2}$ & 22,0 & 7 & 164 & 2 \\
\hline \multirow{6}{*}{$P$} & $\sigma_{p_{1}}^{2}$ & 12800,0 & 21074 & 133040 & 28814 \\
\hline & $\sigma_{p_{12}}$ & 473,0 & 461 & 4707 & 137 \\
\hline & $\sigma_{p_{13}}$ & $-207,8$ & -326 & -1280 & -1811 \\
\hline & $\sigma_{p_{2}}^{2}$ & 20,5 & 38 & 202 & 19 \\
\hline & $\sigma_{p_{23}}$ & $-2,2$ & 6 & 20 & 22 \\
\hline & $\sigma_{p_{3}}^{2}$ & 15,7 & 11 & 171 & 286 \\
\hline \multirow{6}{*}{$\mathrm{R}$} & $\sigma_{e_{1}}^{2}$ & 35200,0 & 115768 & 350966 & 455417 \\
\hline & $\sigma_{e_{12}}$ & 1235,1 & 2564 & 11972 & 15886 \\
\hline & $\sigma_{e_{13}}$ & $-299,5$ & -184 & -3532 & -5116 \\
\hline & $\sigma_{e_{2}}^{2}$ & 51,2 & 155 & 479 & 647 \\
\hline & $\sigma_{e_{23}}$ & 4,6 & 3 & 19 & 2 \\
\hline & $\sigma_{e_{3}}^{2}$ & 40,8 & 128 & 408 & 495 \\
\hline \multicolumn{6}{|c|}{ Informações Adicionais } \\
\hline & 35232 & 10620 & 73983 \\
\hline \multicolumn{3}{|c|}{ № de Iterações } & 2619 & 1096 & 4056 \\
\hline \multicolumn{3}{|c|}{ № de Avaliações } & 3967 & 1 & 6715 \\
\hline \multicolumn{3}{|c|}{ Vet. Par. Inválidos } & 421 & - & 967 \\
\hline \multicolumn{3}{|c|}{ Tentativas } & 4 & 3 & 6 \\
\hline \multicolumn{3}{|c|}{$-2 \log \left(\mathrm{L}_{2}\right)$} & $+t$ & 22119,23 & 23374,17 \\
\hline \multicolumn{3}{|c|}{ № de Registros } & 2742 & 2742 & 2742 \\
\hline \multicolumn{3}{|c|}{ № de Animais } & 1500 & 1407 & 1500 \\
\hline \multicolumn{3}{|c|}{ Clas. Ef. Aleat. Adicionais } & 400 & 400 & 400 \\
\hline \multicolumn{3}{|c|}{ № Class. Ef. Fixos } & 249 & 249 & 249 \\
\hline \multicolumn{3}{|c|}{ № Equações } & 2949 & 952 & 2949 \\
\hline \multicolumn{3}{|c|}{ Esparsivid. das $\mathrm{MME}(\%)$} & 1,38 & - & 2,42 \\
\hline
\end{tabular}


Tabela 17b. Estimativas dos componentes de covariancia para os dados do arquivo SIMUL100.PER, utilizando-se os programas MTC, DFMUV e MTDFRUN. Análise realizada de acordo com a estratégia $\mathbf{B}$ - Com valores perdidos para $\mathrm{y}_{2}$ ou $\mathrm{y}_{3}$

\begin{tabular}{|c|c|c|c|c|c|c|}
\hline $\begin{array}{l}\mathrm{Ma-} \\
\text { triz }\end{array}$ & $\begin{array}{l}\text { Parâ- } \\
\text { metro }\end{array}$ & $\begin{array}{l}\text { Valores } \\
\text { Iniciais }\end{array}$ & $\begin{array}{l}\text { V.Iniciais } \\
\text { para MTC }\end{array}$ & $\begin{array}{r}\text { DFMUV } \\
\text { (1) }\end{array}$ & $\mathrm{MTC}^{+}$ & $\begin{array}{r}\text { MTDFRUN } \\
\text { (1) }\end{array}$ \\
\hline \multirow{6}{*}{ G } & $\sigma_{a_{1}}^{2}$ & *148810 & 143069 & 150963 & 150747 & 159399 \\
\hline & $\sigma_{a_{12}}$ & 5959 & 5959 & 5831 & 6123 & 5990 \\
\hline & $\sigma_{a_{13}}$ & 593 & 593 & 646 & -123 & 596 \\
\hline & $\sigma_{a_{2}}^{2}$ & *335 & 333 & 306 & 324 & 309 \\
\hline & $\sigma_{a_{23}}$ & 156 & 156 & 156 & 103 & 158 \\
\hline & $\sigma_{a_{3}}^{2}$ & $* 198$ & 190 & 217 & 157 & 220 \\
\hline \multirow{6}{*}{$\mathrm{P}$} & $\sigma_{p_{1}}^{2}$ & *147312 & 164980 & 130681 & 158109 & 129422 \\
\hline & $\sigma_{p_{12}}$ & 4730 & 4730 & 4721 & 5644 & 4636 \\
\hline & $\sigma_{p_{13}}$ & -2078 & -2078 & -2040 & -1269 & -2191 \\
\hline & $\sigma_{p_{2}}^{2}$ & $* 220$ & 234 & 201 & 241 & 204 \\
\hline & $\sigma_{p_{23}}$ & -22 & -22 & -32 & 25 & -20 \\
\hline & $\sigma_{p_{3}}^{2}$ & *139 & 134 & 109 & 170 & 146 \\
\hline \multirow{6}{*}{$\mathbf{R}$} & $\sigma_{e_{1}}^{2}$ & *349608 & 349608 & 354179 & 333035 & 355048 \\
\hline & $\sigma_{e_{12}}$ & 12351 & 12351 & 12323 & 11482 & 12398 \\
\hline & $\sigma_{e_{13}}$ & -2995 & -2995 & -3021 & -3107 & -3002 \\
\hline & $\sigma_{e_{2}}^{2}$ & *468 & 468 & 501 & 466 & 503 \\
\hline & $\sigma_{e_{23}}$ & 46 & 46 & 42 & 38 & 38 \\
\hline & $\sigma_{e_{3}}^{2}$ & $* 418$ & 418 & 415 & 420 & 406 \\
\hline \multicolumn{7}{|c|}{ Informações Adicionais } \\
\hline \multicolumn{4}{|c|}{ Tempo(s) } & 21035 & 2383 & 35530 \\
\hline \multicolumn{4}{|c|}{ № de Iterações } & 1327 & 261 & 1855 \\
\hline \multicolumn{4}{|c|}{ № de Avaliações } & 2027 & - & 3587 \\
\hline \multicolumn{4}{|c|}{ Vet. Par. Inválidos } & 292 & - & 744 \\
\hline \multicolumn{4}{|c|}{ Tentativas } & 3 & 1 & 3 \\
\hline \multicolumn{4}{|c|}{$-2 \log \left(\mathrm{L}_{2}\right)$} & 21662,99 & - & 21664,17 \\
\hline \multicolumn{4}{|c|}{ № de Registros } & 2654 & 2478 & 2654 \\
\hline \multicolumn{4}{|c|}{ № de Animais } & 1500 & 1407 & 1500 \\
\hline \multicolumn{4}{|c|}{ Clas. Ef. Aleat. Adicionais } & 400 & 400 & 400 \\
\hline \multicolumn{4}{|c|}{ № Class. Ef. Fixos } & 249 & 249 & 249 \\
\hline \multicolumn{4}{|c|}{ № de Equações } & 2949 & 2856 & 2949 \\
\hline \multicolumn{4}{|c|}{ Esparsividade das MME $(\%)$} & 1,38 & $\cdots$ & 2,34 \\
\hline
\end{tabular}

(1) - Método Simplex + Toda informação é perdida * - Valores fixados na primeira tentativa 
Tabela 17c. Estimativas de herdabilidade $\left(\hat{h}^{2}\right)$, repetibilidade $(\hat{r})$ e correlações genéticas $\left(\hat{r}_{g}\right)$ e de meio ambiente $\left(\hat{r}_{e}\right)$,para os dados das tabelas 17 e $17 \mathrm{~b}$, utilizando-se os programas MTC, DFMUV e MTDFRUN

\begin{tabular}{|l|ccc|ccc|}
\hline \multirow{2}{*}{$\begin{array}{l}\text { Para- } \\
\text { metro }\end{array}$} & \multicolumn{3}{|c|}{ Tabela 17 } & \multicolumn{3}{c|}{ Tabela 17b } \\
\cline { 2 - 7 } & DFMUV & MTC & MT DFRUN & DFMUV & MTC & MTDFRUN \\
\hline$h_{1}^{2}$ & 0,24 & 0,25 & 0,24 & 0,24 & 0,25 & 0,25 \\
$h_{2}^{2}$ & 0,31 & 0,34 & 0,30 & 0,30 & 0,31 & 0,30 \\
$h_{3}^{2}$ & 0,28 & 0,23 & 0,29 & 0,29 & 0,21 & 0,29 \\
\hline$r_{1}$ & 0,44 & 0,46 & 0,44 & 0,44 & 0,48 & 0,45 \\
$r_{2}$ & 0,50 & 0,54 & 0,49 & 0,50 & 0,55 & 0,50 \\
$r_{3}$ & 0,46 & 0,45 & 0,46 & 0,44 & 0,44 & 0,47 \\
\hline$r_{g_{12}}$ & 0,86 & 0,89 & 0,86 & 0,86 & 0,88 & 0,85 \\
$r_{g_{13}}$ & 0,10 & $-0,06$ & 0,11 & 0,10 & $-0,06$ & 0,11 \\
$r_{g_{23}}$ & 0,59 & 0,52 & 0,60 & 0,61 & 0,46 & 0,61 \\
\hline$r_{e_{12}}$ & 0,92 & 0,92 & 0,93 & 0,93 & 0,92 & 0,93 \\
$r_{e_{13}}$ & $-0,24$ & $-0,29$ & $-0,25$ & $-0,25$ & $-0,26$ & $-0,25$ \\
$r_{e_{23}}$ & 0,11 & 0,04 & 0,09 & 0,09 & 0,09 & 0,08 \\
\hline
\end{tabular}

As tabelas de 18 a 18c mostram os resultados da análise dos dados correspondentes aos arquivos MULTI615.ARQ e MULTI615.MIS. Nas avaliações do arquivo MULTI615.ARQ, o programa DFMUV converge irregularmente, ao utilizar-se o método de Powell, e converge adequadamente quando adota-se o método simplex. Neste caso, observa-se número relativamente elevado de vetores de parâmetros inválidos. Fato interessante na análise dos dados desse arquivo é que os valores estimados são divergentes entre os três sistemas. No entanto, todos os resultados levaram ao mesmo valor de $-2 \log \left(\mathrm{L}_{2}\right)$, ao nivel de precisão aqui adotado, considerando-se até a quarta casa decimal. Desta forma, é repetido o processo de estimação para os três programas, adotando-se como valores iniciais para um programa os valores estimados pelos dois outros. Conclui-se que todos os vetores de parâmetros correspondem à conjuntos de valores que levavam ao máximo da função de verossimilhança. Os resultados das análises em forma de estimativas dos parâmetros genéticos e fenotrpicos com respectivos desvios padrão são apresentados na tabela 18a. Verifica-se que os três sistemas estimam adequadamente aos parametros utilizados para simulação.

A perda aleatória de $20 \%$ dos dados para as caracteristicas dois ou três provoca alteração na estrutura de correlação entre as caracterı́sticas. O programa DFMUV estima os mesmos valores estimados no sistema MTDFREML. As diferenças mínimas encontradas são observadas somente a partir da $3^{\text {a }}$ casa decimal e são ignoradas. As variações encontradas entre os valores estimados pelos programas que adotam o método livre de derivadas e o programa MTC são justificadas principalmente por tratar-se de amostras diferentes, dada a perda de registros quando é utilizado o sistema MTC. 
Tabela 18. Estimativas dos componentes de covariancia para os dados do arquivo MULTI615.ARQ, utilizando-se os programas DFMUV,MTC e MTDFRUN. Valores iniciais iguais aos utilizados para simulação.

\begin{tabular}{|c|c|c|c|c|c|c|}
\hline $\begin{array}{l}\text { Ma- } \\
\text { triz }\end{array}$ & $\begin{array}{l}\text { Para- } \\
\text { metro }\end{array}$ & $\begin{array}{l}\text { Valores } \\
\text { Iniciais }\end{array}$ & $\begin{array}{c}\text { DFMUV } \\
(1)\end{array}$ & (2) & $\begin{array}{r}\text { MTC } \\
\quad E M \\
\end{array}$ & $\begin{array}{r}\text { MTDFRUN } \\
(1) \\
\end{array}$ \\
\hline \multirow{6}{*}{ G } & $\sigma_{a_{1}}^{2}$ & 173000 & 152394 & $t+$ & 163811 & 173613 \\
\hline & $\sigma_{a_{12}}$ & 2577 & 2575 & ++ & 2481 & 2500 \\
\hline & $\sigma_{a_{13}}$ & 2325 & 2594 & $+t$ & 2216 & 3304 \\
\hline & $\sigma_{a_{2}}^{2}$ & 240 & 225 & ++ & 171 & 230 \\
\hline & $\sigma_{a_{23}}$ & -52 & -67 & ++ & -54 & -62 \\
\hline & $\sigma_{a_{3}}^{2}$ & 125 & 116 & $+t$ & 137 & 135 \\
\hline \multirow{6}{*}{$P$} & $\sigma_{p_{1}}^{2}$ & 165000 & 163133 & ++ & 157886 & 152058 \\
\hline & $\sigma_{p_{12}}$ & 1795 & 1513 & ++ & 1504 & 1556 \\
\hline & $\sigma_{p_{13}}$ & 968 & 1225 & ++ & 1402 & 878 \\
\hline & $\sigma_{p_{2}}^{2}$ & 217 & 146 & ++ & 176 & 143 \\
\hline & $\sigma_{p_{23}}$ & -17 & -19 & ++ & -24 & -22 \\
\hline & $\sigma_{p_{3}}^{2}$ & 142 & 146 & $+t$ & 137 & 137 \\
\hline \multirow{6}{*}{$\mathbf{R}$} & $\sigma_{e_{1}}^{2}$ & 385000 & 395081 & ++ & 394708 & 395441 \\
\hline & $\sigma_{e_{12}}$ & -4195 & -4983 & ++ & -4946 & -4993 \\
\hline & $\sigma_{e_{13}}$ & -4522 & -4231 & ++ & -4226 & -4238 \\
\hline & $\sigma_{e_{2}}^{2}$ & 508 & 528 & $+t$ & 526 & 528 \\
\hline & $\sigma_{e_{23}}$ & 246 & 258 & ++ & 257 & 258 \\
\hline & $\sigma_{e_{3}}^{2}$ & 332 & 339 & $+t$ & 338 & 339 \\
\hline \multicolumn{7}{|c|}{ Informações Adicionais } \\
\hline \multicolumn{3}{|c|}{ Tempo(s) } & 5634 & $+t$ & 52 & 5528 \\
\hline \multicolumn{3}{|c|}{ № de Iterações } & 1152 & ++ & 11 & 778 \\
\hline \multicolumn{3}{|c|}{ № de Avaliações } & 1607 & ++ & - & 1072 \\
\hline \multicolumn{3}{|c|}{ Vet. Par. Inválidos } & 73 & ++ & - & 13 \\
\hline \multicolumn{3}{|c|}{ Tentativas } & 3 & ++ & 1 & 3 \\
\hline \multicolumn{3}{|c|}{$-2 \log \left(\mathrm{L}_{2}\right)$} & 16826,42 & $+t$ & 16826,43 & 16826,42 \\
\hline \multicolumn{3}{|c|}{ № de Registros } & 1845 & 1845 & 1845 & 1845 \\
\hline \multicolumn{3}{|c|}{ № de Equações } & 1881 & 1881 & 1881 & 1881 \\
\hline \multicolumn{3}{|c|}{ Esparsiv. das MME (\%) } & 0,88 & 0,88 & - & 3,10 \\
\hline
\end{tabular}

(1) - Método Simplex ++ - Convergência Irregular (2) - Método de Powell 
Tabela 18a. Estimativas de herdabilidade $\left(\hat{h}^{2}\right)$, repetibilidade $(\hat{r})$ e correlações genéticas $\left(\hat{r}_{g}\right)$, fenotrpicas $\left(\hat{r}_{p}\right)$ e de meio ambiente $\left(\hat{r}_{e}\right)$, para os dados do arquivo MEYER615. $\mathrm{ARQ}^{+}$utilizando-se os programas DFMUV, MTC e MTDFRUN

\begin{tabular}{||l||c||c||c||c||}
\hline \hline $\begin{array}{l}\text { Para- } \\
\text { metro }\end{array}$ & $\begin{array}{c}\text { Valor do } \\
\text { Parâmetro }\end{array}$ & DFMUV & MTC & MTDFRUN \\
\hline \hline$h_{1}^{2}$ & 0,24 & $0,2233 \pm 0,0148$ & $0,2347 \pm 0,0125$ & $0,2300 \pm 0,0100$ \\
$h_{2}^{2}$ & 0,25 & $0,2517 \pm 0,0039$ & $0,2043 \pm 0,0104$ & $0,2367 \pm 0,0321$ \\
$h_{3}^{2}$ & 0,21 & $0,2032 \pm 0,0161$ & $0,2330 \pm 0,0115$ & $0,2167 \pm 0,0153$ \\
\hline$r_{g_{12}}$ & 0,40 & $0,4270 \pm 0,0256$ & $0,4889 \pm 0,0181$ & $0,4400 \pm 0,0400$ \\
$r_{g_{13}}$ & 0,50 & $0,6337 \pm 0,0364$ & $0,5060 \pm 0,0344$ & $0,5867 \pm 0,1069$ \\
$r_{g_{23}}$ & $-0,30$ & $-0,3990 \pm 0,0412$ & $-0,4017 \pm 0,0458$ & $-0,3700 \pm 0,0346$ \\
\hline$r_{1}$ & 0,46 & $0,4466 \pm 0,0253$ & $0,4507 \pm 0,0229$ & $0,4500 \pm 0,0200$ \\
$r_{2}$ & 0,47 & $0,4128 \pm 0,0066$ & $0,4008 \pm 0,0178$ & $0,4100 \pm 0,0577$ \\
$r_{3}$ & 0,44 & $0,4394 \pm 0,0274$ & $0,4499 \pm 0,0234$ & $0,4434 \pm 0,0268$ \\
\hline$r_{e_{12}}$ & $-0,30$ & $-0,3450 \pm 0,0003$ & $-0,3436 \pm 0,0002$ & $-0,3500 \pm 0,0000$ \\
$r_{e_{13}}$ & $-0,40$ & $-0,3658 \pm 0,0003$ & $-0,3682 \pm 0,0020$ & $-0,3700 \pm 0,0000$ \\
$r_{e_{23}}$ & 0,60 & $0,6110 \pm 0,0001$ & $0,6106 \pm 0,0010$ & $0,6100 \pm 0,0000$ \\
\hline$r_{p_{12}}$ & 0,01 & $-0,0356 \pm 0,0007$ & $-0,0337 \pm 0,0051$ & $-0,0359 \pm 0,0014$ \\
$r_{p_{13}}$ & 0,06 & $-0,0250 \pm 0,0086$ & $-0,0219 \pm 0,0066$ & $-0,0244 \pm 0,0050$ \\
$r_{p_{23}}$ & 0,23 & $0,2334 \pm 0,0014$ & $0,2371 \pm 0,0068$ & $0,2363 \pm 0,0051$ \\
\hline \hline
\end{tabular}

t - Resultado de três repetições 
Tabela 18b. Estimativas dos componentes de covariancia para os dados do arquivo MULTI615.MIS, utilizando-se os programas MTC, DFMUV e MTDFRUN. Valores iniciais iguais aos utilizados para simulação. $20 \%$ de dados perdidos para $\mathrm{y}_{2}$ ou $\mathrm{y}_{3}$

\begin{tabular}{|c|c|c|c|c|c|}
\hline $\begin{array}{l}\text { Ma- } \\
\text { triz }\end{array}$ & $\begin{array}{l}\text { Parâ- } \\
\text { metro }\end{array}$ & $\begin{array}{l}\text { Valores } \\
\text { Iniciais }\end{array}$ & $\begin{array}{r}\text { DFMUV } \\
\text { (1) }\end{array}$ & $\begin{array}{r}\mathrm{MTC}^{+} \\
\mathrm{EM}\end{array}$ & $\begin{array}{r}\text { MTDFRUN } \\
\text { (1) }\end{array}$ \\
\hline \multirow{6}{*}{ G } & $\sigma_{a_{1}}^{2}$ & 173000 & 160978 & 148071 & 160978 \\
\hline & $\sigma_{a_{12}}$ & 2577 & 2172 & 3139 & 2172 \\
\hline & $\sigma_{a_{13}}$ & 2325 & 2712 & 2070 & 2712 \\
\hline & $\sigma_{a_{2}}^{2}$ & 240 & 286 & 164 & 286 \\
\hline & $\sigma_{a_{23}}$ & -52 & -87 & -43 & -87 \\
\hline & $\sigma_{a_{3}}^{2}$ & 125 & 159 & 123 & 159 \\
\hline \multirow{6}{*}{$\mathbf{P}$} & $\sigma_{p_{1}}^{2}$ & 165000 & 158532 & 128595 & 158532 \\
\hline & $\sigma_{p_{12}}$ & 1795 & 1793 & 2534 & 1793 \\
\hline & $\sigma_{p_{13}}$ & 968 & 1456 & 1667 & 1456 \\
\hline & $\sigma_{p_{2}}^{2}$ & 217 & 98 & 144 & 98 \\
\hline & $\sigma_{p_{23}}$ & -17 & -15 & -31 & -15 \\
\hline & $\sigma_{p_{3}}^{2}$ & 142 & $\begin{array}{r}69 \\
\end{array}$ & 107 & 69 \\
\hline \multirow{6}{*}{$\mathrm{R}$} & $\sigma_{e_{1}}^{2}$ & 385000 & 395046 & 376614 & 395046 \\
\hline & $\sigma_{e_{12}}$ & -4195 & -5194 & -5840 & -5194 \\
\hline & $\sigma_{e_{13}}$ & -4522 & -4823 & -4856 & -4823 \\
\hline & $\sigma_{e_{2}}^{2}$ & 508 & 563 & 577 & 563 \\
\hline & $\sigma_{e_{23}}$ & 246 & 285 & 296 & 285 \\
\hline & $\sigma_{e_{3}}^{2}$ & 332 & 376 & 386 & 376 \\
\hline \multicolumn{6}{|c|}{ Informaçôes Adicionais } \\
\hline \multicolumn{3}{|c|}{ Tempo(s) } & 2916 & 107 & 8702 \\
\hline \multicolumn{3}{|c|}{ № de Iterações } & 613 & 36 & 1255 \\
\hline \multicolumn{3}{|c|}{ № de Avaliações } & 938 & - & 1792 \\
\hline \multicolumn{3}{|c|}{ № de Vet. Par. Inválidos } & 0 & - & 35 \\
\hline \multicolumn{3}{|c|}{ № de Tentativas } & 2 & 1 & 5 \\
\hline \multicolumn{3}{|c|}{$-2 \log \left(\mathrm{L}_{2}\right)^{\#}$} & 15197,79 & 15202,15 & 15197,79 \\
\hline \multicolumn{3}{|c|}{ № de Registros } & $\therefore 1612$ & 1230 & 1612 \\
\hline \multicolumn{3}{|c|}{ № de Equações } & 1881 & 1680 & 1881 \\
\hline \multicolumn{3}{|c|}{ Esparsividade das MME (\%) } & 1,22 & - & 2,79 \\
\hline
\end{tabular}

(1) - Método Simplex 
Tabela 18c. Estimativas de herdabilidade $\left(\hat{h}^{2}\right)$, repetibilidade $(\hat{r})$ e correlações genéticas $\left(\hat{r}_{g}\right)$, fenotipicas $\left(\hat{r}_{p}\right)$ e de meio ambiente $\left(\hat{r}_{e}\right)$, para os dados da tabela $18 \mathrm{~b}$., utilizando-se os programas DFMUV, MTDFRUN e MTC

\begin{tabular}{|l|c|c|c|}
\hline Parâ- & Valor do & DFMUV e & MTC \\
metro & Parámetro & MTDFRUN & 0,23 \\
\hline$h_{1}^{2}$ & 0,24 & 0,23 & 0,19 \\
$h_{2}^{2}$ & 0,25 & 0,30 & 0,20 \\
$h_{3}^{2}$ & 0,21 & 0,26 & 0,64 \\
\hline$r_{g_{12}}$ & 0,40 & 0,32 & 0,49 \\
$r_{g_{13}}$ & 0,50 & 0,54 & $-0,31$ \\
$r_{g_{23}}$ & $-0,30$ & $-0,41$ & 0,42 \\
\hline$r_{1}$ & 0,46 & 0,45 & 0,35 \\
$r_{2}$ & 0,47 & 0,41 & 0,37 \\
$r_{3}$ & 0,44 & 0,38 & $-0,00$ \\
\hline$r_{p_{12}}$ & 0,01 & 0,05 & $-0,06$ \\
$r_{p_{13}}$ & 0,06 & $-0,03$ & 0,30 \\
$r_{p_{23}}$ & 0,23 & 0,24 & $-0,40$ \\
\hline$r_{e_{12}}$ & $-0,30$ & $-0,35$ & $-0,40$ \\
$r_{e_{13}}$ & $-0,40$ & $-0,40$ & 0,63 \\
$r_{e_{23}}$ & 0,60 & 0,62 & \\
\hline
\end{tabular}

As análises do arquivo SIMUL400.ARQ (Tabelas de 19 a 19b) são realizadas especialmente para analisar o comportamento dos sistemas frente a um volume maior de dados simulados. Para este conjunto de dados o sistema MTC embora alcance convergencia em tempo muito inferior aos demais programas, os valores por ele estimados não correspondem ao máximo da função de verossimilhança ou mínimo de $-2 \log \left(\mathrm{L}_{2}\right)$. A diferença neste caso foi relativamente alta, todavia, não resultam em grandes diferenças nos valores estimados dos parâmetros genéticos e fenotípicos.

Assim, em casos de modelos com mais de um fator aleatório em que se esperam maiores diferenças das estimativas obtidas pelos programas MTC e os que adotam método livre de derivadas, fica a critério do pesquisador. Ou opta por estimativas que resultem em valor máximo da função de verossimilhança, mas demandam tempo de computador muito maior ou opta pelo programa MTC que, embora aproximado, converge em tempo inferior. A decisão dependerá dos objetivos do trabalho.

Para estimação de componentes de covariancia adotando-se o método de Powell, a matriz de coeficientes torna-se não PD. É encontrado raiz de zero ou de valor negativo durante o processo de fatoração. Desta forma, os resultalos não são apresentados.

A perda de dados, para amostra de maior volume não provoca alteração significativa na estrutura de covariância nas diferentes matrizes, mostrando que possiveis alterações observadas em outras avaliações podem ser atriburdas especialmente a problema de amostragem, isto é, amostra de tamanho pequeno.

Vale ressaltar nesta análise o tempo excessivo gasto para estimar os componentes de covariancia ao adotar-se o método livre de derivadas ( $21 \mathrm{~h} \mathrm{e} 15 \mathrm{~min}$. para o 
DFMUV e $45 \mathrm{~h}$ e $34 \mathrm{~min}$. para o sistema MTDFREML).

Tabela 19. Estimativas dos componentes de covariancia para os dados do arquivo SIMUL400.ARQ, utilizando-se os programas MTC, DFMUV e MTDFRUN. Valores iniciais baseados nos resultados das análises uni e bivariada. - sem perda de dados

\begin{tabular}{|c|c|c|c|c|c|}
\hline $\begin{array}{l}\text { Ma- } \\
\text { triz }\end{array}$ & $\begin{array}{l}\text { Parâ- } \\
\text { metro }\end{array}$ & $\begin{array}{l}\text { Valores } \\
\text { Iniciais }\end{array}$ & $\begin{array}{r}\text { DFM UV } \\
\text { (1) }\end{array}$ & $\begin{array}{r}M T C \\
E M \\
\end{array}$ & $\begin{array}{r}\text { MTDFRUN } \\
(1)\end{array}$ \\
\hline \multirow{6}{*}{$G$} & $\sigma_{a_{1}}^{2}$ & 158742 & 161116 & 185647 & 161725 \\
\hline & $\sigma_{a_{12}}$ & 3037 & 3144 & 3111 & 3318 \\
\hline & $\sigma_{a_{13}}$ & 3990 & 3752 & 4133 & 3871 \\
\hline & $\sigma_{a_{2}}^{2}$ & 271 & 269 & 231 & 274 \\
\hline & $\sigma_{a_{23}}$ & 111 & 122 & 111 & 126 \\
\hline & $\sigma_{a_{3}}^{2}$ & 126 & 129 & 132 & 131 \\
\hline \multirow{6}{*}{$\mathrm{P}$} & $\overline{\sigma_{a_{1}}^{2}}$ & 181953 & 182640 & 161504 & 180734 \\
\hline & $\sigma_{a_{12}}$ & 3946 & 3921 & 3928 & 3868 \\
\hline & $\sigma_{a_{13}}$ & 4297 & 4509 & 4149 & 4467 \\
\hline & $\sigma_{a_{2}}^{2}$ & 164 & 165 & 196 & 164 \\
\hline & $\sigma_{a_{23}}$ & 126 & 117 & 126 & 116 \\
\hline & $\sigma_{a_{3}}^{2}$ & 138 & 136 & 132 & 135 \\
\hline \multirow{6}{*}{$\mathrm{R}$} & $\sigma_{e_{1}}^{2}$ & 384517 & 384860 & 386147 & 384479 \\
\hline & $\sigma_{e_{12}}$ & 10472 & 10481 & 10456 & 10481 \\
\hline & $\sigma_{e_{13}}$ & 10311 & 10328 & 10341 & 10318 \\
\hline & $\sigma_{e_{2}}^{2}$ & 501 & 501 & 497 & 501 \\
\hline & $\sigma_{e_{23}}$ & 333 & 336 & 334 & 336 \\
\hline & $\sigma_{e_{3}}^{2}$ & 330 & 332 & 331 & 332 \\
\hline \multicolumn{6}{|c|}{ Informações Adicionais } \\
\hline \multicolumn{3}{|c|}{ Tempo(s) } & 76500 & 8006 & 164042 \\
\hline \multicolumn{3}{|c|}{ № de Iterações } & 1349 & 131 & 1695 \\
\hline \multicolumn{3}{|c|}{ № de Avaliações } & 1855 & - & 2301 \\
\hline \multicolumn{3}{|c|}{ Vet. Par. Inválidos } & 1 & - & 11 \\
\hline \multicolumn{3}{|c|}{ Tentativas } & 3 & 2 & 3 \\
\hline \multicolumn{3}{|c|}{$-2 \log \left(\mathrm{L}_{2}\right)$} & 96084,74 & 96088,27 & 96084,74 \\
\hline \multicolumn{3}{|c|}{ № de Registros } & 11139 & 11139 & 11139 \\
\hline \multicolumn{3}{|c|}{ № de Equações } & 10498 & 10497 & 10569 \\
\hline \multicolumn{3}{|c|}{ Esparsiv. das MME (\%) } & 0,44 & - & 0,74 \\
\hline
\end{tabular}

(1) - Método Simplex

EM - Maximização de Esperanças 
Tabela 19a. Estimativas dos componentes de covariancia para os dados do arquivo SIMUL400.ARQ, utilizando-se os programas MTC, DFMUV e MTDFRUN. Valores iniciais baseados nos resultados das análises uni e bivariada. - Perda de 20\% dos dados para $y_{2}$ ou $y_{3}$

\begin{tabular}{|c|c|c|c|c|c|}
\hline $\begin{array}{l}\text { Ma- } \\
\text { triz }\end{array}$ & $\begin{array}{l}\text { Parâ- } \\
\text { metro }\end{array}$ & $\begin{array}{l}\text { Valores } \\
\text { Iniciais }\end{array}$ & $\begin{array}{r}\text { DFMUV } \\
(1)\end{array}$ & $\begin{array}{r}\mathrm{MTC}^{+} \\
\mathrm{EM}\end{array}$ & $\begin{array}{r}\text { MTDFRUN } \\
\text { (1) }\end{array}$ \\
\hline \multirow{6}{*}{ G } & $\overline{\sigma_{a_{1}}^{2}}$ & 158742 & 152407 & 186779 & 148031 \\
\hline & $\sigma_{a_{12}}$ & 3037 & 2647 & 3019 & 2431 \\
\hline & $\sigma_{a_{13}}$ & 3990 & 3524 & 4181 & 3397 \\
\hline & $\sigma_{a_{2}}^{2}$ & 271 & 254 & 244 & 247 \\
\hline & $\sigma_{a_{23}}$ & 111 & 114 & 109 & 108 \\
\hline & $\sigma_{a_{3}}^{2}$ & 126 & 124 & 132 & 120 \\
\hline \multirow{6}{*}{$\mathbf{P}$} & $\sigma_{a_{1}}^{2}$ & 181953 & 188818 & 163792 & 192411 \\
\hline & $\sigma_{a_{12}}$ & 3946 & 4312 & 4174 & 4488 \\
\hline & $\sigma_{a_{13}}$ & 4297 & 4656 & 4298 & 4762 \\
\hline & $\sigma_{a_{2}}^{2}$ & 164 & 180 & 199 & 187 \\
\hline & $\sigma_{a_{23}}$ & 126 & 123 & 132 & 128 \\
\hline & $\sigma_{a_{3}}^{2}$ & 138 & 138 & 136 & 141 \\
\hline \multirow{6}{*}{$\mathbf{R}$} & $\overline{\sigma_{e_{1}}^{2}}$ & 384517 & 380041 & 394521 & 380234 \\
\hline & $\sigma_{e_{12}}$ & 10472 & 10346 & 10579 & 10348 \\
\hline & $\sigma_{e_{13}}$ & 10311 & 10226 & 10582 & 10228 \\
\hline & $\sigma_{e_{2}}^{2}$ & 501 & 494 & 490 & 494 \\
\hline & $\sigma_{e_{23}}$ & 333 & 333 & 336 & 333 \\
\hline & $\sigma_{e_{3}}^{2}$ & 330 & 330 & 339 & 330 \\
\hline \multicolumn{6}{|c|}{ Informações Adicionais } \\
\hline \multicolumn{3}{|c|}{ Tempo(s) } & 161435 & 728 & 185662 \\
\hline \multicolumn{3}{|c|}{$N^{\circ}$ de Iterações } & 2492 & 21 & 2166 \\
\hline \multicolumn{3}{|c|}{ № de Avaliações } & 3507 & - & 3080 \\
\hline \multicolumn{3}{|c|}{ Vet. Par. Inválidos } & 4 & - & 22 \\
\hline \multicolumn{3}{|c|}{ Tentativas } & 4 & 1 & 6 \\
\hline \multicolumn{3}{|c|}{$-2 \log \left(\mathrm{L}_{2}\right)^{\#}$} & 87388,40 & 87395,22 & 87388,52 \\
\hline \multicolumn{3}{|c|}{ № de Registros } & 9654 & 7056 & 9654 \\
\hline \multicolumn{3}{|c|}{ № de Equações } & 10498 & 9276 & 10569 \\
\hline \multicolumn{3}{|c|}{ Esparsiv. das MME (\%) } & 0,50 & - & 0,64 \\
\hline \multirow{2}{*}{\multicolumn{4}{|c|}{$\begin{array}{l}\text { (1) - Método Simplex } \\
\text { EM - Maximização de Esper }\end{array}$}} & mparável en & MTC e DF \\
\hline & & & & + - Todo reg & tro é perdido \\
\hline
\end{tabular}


Tabela 19b. Estimativas de herdabilidade $\left(\hat{h}^{2}\right)$, repetibilidade $(\hat{r})$ e correlações genéticas $\left(\hat{r}_{g}\right)$, fenotípicas $\left(\hat{r}_{p}\right)$ e de meio ambiente $\left(\hat{r}_{e}\right)$, para os dados das tabelas 19 e 19a., utilizando-se os programas DFMUV, MTC e MTDFRUN

\begin{tabular}{|l|ccc|ccc|}
\hline \multirow{2}{*}{$\begin{array}{l}\text { Pará- } \\
\text { metro }\end{array}$} & \multicolumn{3}{|c|}{ Tabela 19 } & \multicolumn{3}{c|}{ Tabela 19a } \\
\cline { 2 - 7 } & DFMUV & MTC & MTDF RUN & DFMUV & MTC & MTDFRUN \\
\hline$h_{1}^{2}$ & 0,22 & 0,25 & 0,23 & 0,21 & 0,25 & 0,21 \\
$h_{2}^{2}$ & 0,29 & 0,25 & 0,29 & 0,27 & 0,26 & 0,27 \\
$h_{3}^{2}$ & 0,22 & 0,22 & 0,22 & 0,21 & 0,22 & 0,20 \\
\hline$r_{1}$ & 0,47 & 0,47 & 0,47 & 0,47 & 0,47 & 0,48 \\
$r_{2}$ & 0,46 & 0,46 & 0,46 & 0,47 & 0,47 & 0,47 \\
$r_{3}$ & 0,44 & 0,44 & 0,44 & 0,44 & 0,44 & 0,44 \\
\hline$r_{g_{12}}$ & 0,48 & 0,47 & 0,49 & 0,43 & 0,45 & 0,40 \\
$r_{g_{13}}$ & 0,82 & 0,84 & 0,83 & 0,81 & 0,84 & 0,80 \\
$r_{g_{23}}$ & 0,66 & 0,64 & 0,67 & 0,64 & 0,61 & 0,62 \\
\hline$r_{p_{12}}$ & 0,67 & 0,67 & 0,67 & 0,67 & 0,67 & 0,67 \\
$r_{p_{13}}$ & 0,89 & 0,89 & 0,89 & 0,89 & 0,90 & 0,89 \\
$r_{p_{23}}$ & 0,77 & 0,77 & 0,77 & 0,77 & 0,77 & 0,77 \\
\hline$r_{e_{12}}$ & 0,75 & 0,75 & 0,75 & 0,75 & 0,76 & 0,75 \\
$r_{e_{13}}$ & 0,91 & 0,91 & 0,91 & 0,91 & 0,91 & 0,91 \\
$r_{e_{23}}$ & 0,82 & 0,82 & 0,82 & 0,82 & 0,82 & 0,82 \\
\hline
\end{tabular}

As tabelas 20 e $20 \mathrm{a}$. apresentam os resultados das análises dos dados reais do arquivo CNPGL001.ARQ sob o enfoque multivariado. As três características avaliadas são as produções de leite até 305 dias de lactação no $1^{\circ}, 2^{\circ}$ e $3^{\circ}$ partos. Para este tipo de dados, bom número de animais (vacas) apresentam registro de produção no 1ำ parto, mas não no $2^{\circ}$, devido, em geral, à eliminação por baixa produção ou outro problema verificado. Além disso, novas vacas são eliminadas do $2^{\circ}$ para o $3^{\circ}$ parto. Desta forma, há um grande desbalanceamento gerado para esse tipo de dados. No caso presente, 1718 vacas apresentaram dados de produção no $1^{\circ}$ parto, das quais apenas $1142(66,47 \%)$ o fizeram ao $2^{\circ}$ parto e apenas $839(48.84 \%)$ produziram no $3^{\circ}$ parto. Assim, programas que requerem igualdade de matrizes delineamento para as diversas características apresentam resultados comprometidos, devido ao pequeno tamanho relativo da amostra utilizada após eliminação dos registros com dados perdidos. O programa MTC no presente caso considera apenas 763 observações por característica, perfazendo um total de 2289 registros totais. Por outrolado, os programas que adotam o método livre de derivadas consideram um total de 3699 registros. Portanto, para situações similares, espera-se, no mínimo maior precisão das estimativas obtidas pelo método livre de derivadas em relação ao algorftmo EM-REML, com transformação canônica do vator de observações, aqui apresentado e representado pelo programa MTC.

Assim, como esperado, há diferenças nos valores estimados dos componentes de covariancia e dos parâmetros genéticos e fenotípicos para os programas MTC, 
DFMUV e MTDFREML. Os dois últimos convergem para valores similares, mas o sistema MTDFREML, embora muito mais lento, estima componentes de covariancia que correspondem à função de verossimilhança restrita maior, ou seja são valores que realmente minimizaram $-2 \log \left(\mathrm{L}_{2}\right)$. Os valores estimados pelo programa DFMUV, mesmo após 4 (quatro) repetições do processo correspondem apenas a uma aproximação do máximo da função de verossimilhança.

Constata-se decréscimo do coeficiente de herdabilidade da produção de leite do $1^{\circ}$ para o $2^{\circ}$ partos e novo aumento do $2^{\circ}$ para o $3^{\circ}$ partos, quando adotam-se os sistemas DFREML e MTDFREML. Ao contrário, o sistema MTC detecta aumentos crescentes desse coeficiente entre o $1^{\circ}$ e $3^{\circ}$ partos $(0,08,0,11$ e 0,25$)$. Como esperado, observam-se altas correlações genéticas entre as produções de leite nos três primeiros partos. Isto significa que, geneticamente, o uso da produção de leite de uma única lactação de uma vaca é suficiente para realizar a sua avaliação genética. A correlação genética das produções nos $2^{\circ}$ e $3^{\circ}$ partos foi próxima da unidade. MEYER (1993) sugere que nesses casos é recomendável estimar os componentes de covariancia fixando-se o coeficiente à unidade e estima-se os demais, o que poderá aumentar a eficiencia do processo de estimação adotando-se os métodos livres de derivadas. Os programas do sistema DFREML oferecem esta opção de processamento, mas não os sistemas MTDFREML ou MTC. Por isso, neste trabalho, esta alternativa não foi utilizada.

As análises univariadas do mesmo arquivo de dados, cujos resultados são apresentados nas tabelas 10 até 10c. mostram estimativas do coeficiente de herdabilidade da ordem de $0,12 \pm 0,04$, adotando-se um modelo animal univariado e de 0,14 para um modelo de reprodutor. Para os dois casos a repetibilidade estimada para a produção de leite foi da ordem de $\mathbf{0 , 4 6}$. Desta forma, os coeficientes de herdabilidade estimados em análise univariada correspondem a uma média dos valores observados em análise multivariada.

Nota-se redução substancial da variância genética aditiva da produção de leite do $1^{\circ}$ para o $2^{\circ}$ parto quando se adota o modelo animal e o método livre de derivadas em análise univariada. Este resultado está relacionado à forte seleção de animais, que é praticada do primeiro para o segundo parto, baseada na produção de leite. Isto é um fato e necessita ser melhor explorado em trabalhos especfficos. A análise multivariada não mostra tal decréscimo em variância genética aditiva, confirmando a efetividade do método REML em eliminar ou reduzir o viés advindo da seleção de animais (MEYER, 1983). 
Tabela 20. Estimativas dos componentes de covariancia para os dados do arquivo CNPGL001.ARQ, utilizando-se os programas DFMUV, MTC e MTDFRUN. Análise executada de acordo com a estratégia $B$. Existem valores perdidos

\begin{tabular}{|c|c|c|c|c|c|c|}
\hline $\begin{array}{l}\mathrm{Ma}- \\
\text { triz }\end{array}$ & $\begin{array}{l}\text { Para- } \\
\text { metro }\end{array}$ & $\begin{array}{l}\text { Valores } \\
\text { Iniciais }\end{array}$ & $\begin{array}{l}\text { V.Iniciais } \\
\text { para MTC }\end{array}$ & $\begin{array}{r}\text { DFMUV }^{+} \\
(1)\end{array}$ & $\begin{array}{r}\text { MTC } \\
\text { EM } \\
\end{array}$ & $\begin{array}{r}\text { MTDFRUN } \\
(1) \\
\end{array}$ \\
\hline \multirow{6}{*}{ G } & $\overline{\sigma_{a_{1}}^{2}}$ & 43677 & 8659 & 40153 & 18024 & 42217 \\
\hline & $\sigma_{a_{12}}$ & 28589 & 10757 & 35786 & 14601 & 35786 \\
\hline & $\sigma_{a_{13}}$ & 39846 & 23900 & 35829 & 19355 & 40488 \\
\hline & $\sigma_{a_{2}}^{2}$ & 29240 & 20879 & 44263 & 37846 & 44695 \\
\hline & $\sigma_{a_{23}}$ & 32602 & 37000 & 53492 & 59802 & 53492 \\
\hline & $\sigma_{a_{3}}^{2}$ & 56798 & 103081 & 69551 & 95651 & 64426 \\
\hline \multirow{6}{*}{$\mathrm{R}$} & $\sigma_{e_{1}}^{2}$ & 203092 & 220204 & 206888 & 211594 & 204526 \\
\hline & $\sigma_{e_{12}}$ & 132988 & 136000 & 142367 & 144619 & 144538 \\
\hline & $\sigma_{e_{13}}$ & 135471 & 129787 & 94019 & 100771 & 99048 \\
\hline & $\sigma_{e_{2}}^{2}$ & 348334 & 336098 & 321444 & 319836 & 344410 \\
\hline & $\sigma_{e_{23}}$ & 177418 & 160344 & 169702 & 158532 & 188234 \\
\hline & $\sigma_{e_{3}}^{2}$ & 361459 & 305987 & 344959 & 312304 & 361012 \\
\hline \multicolumn{7}{|c|}{ Informações Adicionais } \\
\hline \multicolumn{4}{|c|}{ Tempo(s) } & 28927 & 772 & 62789 \\
\hline \multicolumn{4}{|c|}{ № de Iterações } & 884 & 121 & 2207 \\
\hline \multicolumn{4}{|c|}{ № de Avaliações } & 1285 & - & 3863 \\
\hline \multicolumn{4}{|c|}{$N^{\circ}$ Vet. Par. Inválidos } & 265 & - & 890 \\
\hline \multicolumn{4}{|c|}{ № de Tentativas } & 4 & 1 & 7 \\
\hline \multicolumn{4}{|c|}{$-2 \log \left(L_{2}\right)^{\#}$} & 40011,43 & 22411,15 & 40007,69 \\
\hline \multicolumn{4}{|c|}{ № de Registros } & 3699 & 2289 & 3699 \\
\hline \multicolumn{4}{|c|}{ № de Animais } & 2365 & 2289 & 2365 \\
\hline \multicolumn{4}{|c|}{ № Ef. Aleatórios Adicionais } & 0 & 0 & 0 \\
\hline \multicolumn{4}{|c|}{ № Class. Ef. Fixos } & 797 & 669 & 797 \\
\hline \multicolumn{4}{|c|}{ № de Equações } & 7892 & 6568 & 7892 \\
\hline \multicolumn{4}{|c|}{ Esparsividade das MME (\%) } & 0,42 & - & 0,43 \\
\hline \multicolumn{4}{|c|}{ (1) - Método Simplex } & E & Iaximizac & Esperanças \\
\hline
\end{tabular}


Tabela 20a. Estimativas de herdabilidade $\left(\hat{h}^{2}\right)$ e correlações genéticas $\left(\hat{r}_{g}\right)$, fenotípicas $\left(\hat{r}_{p}\right)$ e de meio ambiente $\left(\hat{r}_{e}\right)$, para os dados da tabela 20, utilizando-se os programas DFMUV, MTC e MTDFRUN

\begin{tabular}{|l|c|c|c|}
\hline Parámetro & DFMUV & MTC & MTDFRUN \\
\hline$h_{1}^{2}$ & 0,16 & 0,08 & 0,17 \\
$h_{2}^{2}$ & 0,12 & 0,11 & 0,12 \\
$h_{3}^{2}$ & 0,17 & 0,23 & 0,15 \\
\hline$r_{g_{12}}$ & 0,85 & 0,56 & 0,82 \\
$r_{g_{13}}$ & 0,68 & 0,47 & 0,78 \\
$r_{g_{23}}$ & 0,96 & 0,99 & 0,99 \\
\hline$r_{p_{12}}$ & 0,59 & 0,56 & 0,58 \\
$r_{p_{13}}$ & 0,35 & 0,39 & 0,36 \\
$r_{p_{23}}$ & 0,57 & 0,39 & 0,59 \\
\hline$r_{e_{12}}$ & 0,55 & 0,56 & 0,54 \\
$r_{e_{13}}$ & 0,35 & 0,39 & 0,36 \\
$r_{e_{23}}$ & 0,51 & 0,50 & 0,53 \\
\hline \multicolumn{3}{|c|}{ Informaçóes Adicionais } \\
\hline \multicolumn{3}{|c|}{ Caracteristica } \\
\hline \multicolumn{3}{|c|}{ P305 } & P3053 \\
\hline Média(kg) & 2107,49 & 2540,06 & 2624,33 \\
DP(kg) & 561,64 & 710,95 & 761,46 \\
CV(\%) & 26,65 & 27,99 & 29,02 \\
N & 1718 & 1142 & 839 \\
\hline
\end{tabular}




\section{CONCLUSÕES}

1. Em análise multivariada, o máximo da função de verossimilhança pode ser encontrado por diferentes combinações de valores estimados dos componentes de covariancia;

2. Estimação de componentes de covariancia em análise multivariada constitui-se em tarefa complexa, onde o valor estimado de um componente não tem valor por si só, mas o que realmente deve merecer destaque é a combinação de componentes e os resultados ou efeitos que esses possam produzir;

3. A convergência dos métodos iterativos, empregados na estimação de componentes de covariancia, em análise multivariada, depende dos valores iniciais adotados e da estrutura de correlação das matrizes de componentes a serem estimados e do tipo de superfície a ser considerada. Quanto maior o número de condição das matrizes, maiores as dificuldades em se encontrar o máximo da função de verossimilhança e maior o risco de se obter estimativas viciadas;

4. Procedimentos que adotam os métodos livres de derivadas, na estimação de componentes de variância (análise univariada), são muito eficientes na procura do máximo da função de verossimilhança e produzem estimativas invariantes sob diferentes valores preliminares adotados;

5. Procedimentos que adotam os métodos livres de derivadas na estimação de componentes de covariância, em análise multivariada, podem ser eficientes. A eficiência depende da utilização de valores preliminares adequados (próximos à convergência), várias repetições do processo, se possı́vel com diferentes valores iniciais adequados;

6. A perda de dados em análise multivariada pode causar alterações na estrutura de covariancias entre características;

7. O método de Powell, ou método das direções conjugadas, mostra-se muito eficiente na procura de mínimos de funções em a nálise univariada. Em análise multivariada, a eficiência do método depende do tipo de função que se está tentando minimizar. Em geral, o método tende a apresentar convergência prematura ou irregular quando a superf́cie de verossimilhança é muito plana, as matrizes são mal condicionadas ou as estimativas estão próximas ao limite do espaço de parâmetros; 
8. O método simplex mostra-se adequado e robusto na procura de mínimos de funções multiparamétricas, para modelos com um ou mais fatores aleatórios não correlacionados entre si, embora seja um método mais lento do que o método de Powell;

9. O algorftmo EM-REML, utilizado no programa MTC, apresenta-se como uma excelente alternativa para estimação de com ponentes de covariancia de uma forma rápida, em análises de uma classe restrita de modelos mistos e merece ser trabalhado com o objetivo de se conseguir a sua generalização, para modelos mais gerais;

10. Avaliação e interpretação de resultados de análises de dados da área zootécnica merecem atenção devida, tendo em vista que os valores estimados dependem, entre outros, da amostra utilizada, do modelo adotado, do método empregado, do critério de seleção/eliminação de dados e do tipo de análise se uni ou multivariada;

11. Os sistemas DFREML e MTDFREML são similares. Os programas do sistema DFREML são, no geral, mais rápidos para atingir a convergência. o MTDFREML é mais lento, mas parece utilizar algum algorftmo mais refinado com objetivo de prever matrizes fora do espaço paramétrico;

12. Os programas que adotam o método livre de derivadas apresentaram a desvantagem de serem muito lentos para análises em que envolvam número elevado de características e classes de efeitos fixos e aleatórios, MME não esparsas, matrizes mal condicionadas e convergência próxima ao limite do espaço de parâmetros;

13. Estimativas de parâmetros genéticos ou fenotípicos em geral não são as mesmas quando estimadas por análise uni ou multivariada. Desta forma, o uso destas dependerá do critério de seleção adotado. Se a seleção for baseada em características individuais, então deve-se adotar os parâmetros obtidos em análise univariada. Caso contrário, deve-se utilizar as estimativas obtidas em análise incluindo todas características sob seleção.

14. No geral, os sistemas DFREML, MTC e MTDFREML constituem-se em ótimas ferramentas para estimação de componente de covariancia para modelos bem gerais, com grande número de classes de efeitos fixos e aleatórios. 


\section{REFERENCIAS BIBLIOGRÁFICAS}

ANDREONI, S. Modelos de efeitos aleatórios para análise de dados longitudinais não balanceados em relação ao tempo. São Paulo, 1989. 142p. (Mestrado - Instituto de Matematica e Estatistica/USP).

BOLDMAN, K.G. \& VAN VLECK, L.D. Derivative-free restricted maximum likelihood estimation in animal models with a sparse matrix solver. Journal of Dairy Science, Champaign, 74(12): 4337-43, 1991.

BOLDMAN, K.G.; KRIESE, L.A.; VAN VLECK, L.D.; KACHMAN, S.D. A Manual for use of MTDFREML; a set of programs to obtain estimates of variances and covariances [DRAFT]. Lincoln, Department of Agriculture/Agricultural Research Service, 1993. 120p.

BOX, G.E.P. \& MILLER, M.E. A note on the generation of random normal deviates. Annals of Mathematics Statistics, Hayward, 29 : 610-1, 1958.

BROWN, K.G. Asymptotic behavior of MINQUE - type estimators of variance components. Annals of Statistics, Hayward, 4: 746-54, 1976.

CALVIN, J. A. REML estimation in unbalanced multivariate variance components models using an EM algorithm. Biometrics, Washington, 49(3): 691-702, 1993.

CHAUHAN, V.P.S. \& HAYES, J.F. Genetic parameters for first lactation milk production and composition traits for Holsteins using multivariate restricted maximum likelihood. Journal of Dairy Science, Champaign, 74 (2): 603-10, 1991.

CONTE, S.D. Elementos de análise numérica. 3. ed. Porto Alegre, Globo, 1977. $331 p$.

CORBEIL, R.R. \& SEARLE, S.R. A comparison of variance component estimators. Biometrics, Washington, 32: 779-91, Dec. 1976.

CUNNINGHAM, E.P. \& HENDERSON, C.R. An iterative procedure for estimating fixed effects and variance components in mixed model situations. Biometrics, Washington, 24: 13-6, 1968. 
DA, Y.; GROSSMAN, M.; MISZTAL, I. Prediction error variance and restricted maximum likelihood estimation for animal model with relationship grouping. Journal of Dairy Science, Champaign, 72 (8): 2125-35,1989.

DEMPSTER, A.P.; LAIRD, N.M.; RUBIN, D.B. Maximum likelihood from incomplete data via the EM algorithm. Journal of the Royal Statistical Society. Ser. B., Oxford, 39 : 1-38, 1977.

GEORGE, A. \& LIU, J.W.H. Computer solution of large positive definite systems. New Jersey, Prentice-Hall, 1981. 180p.

GEORGE, A.; LIU, J.W.H; NG, E. User guide for SPARSPAK: Waterloo sparse linear equations package. Ontario, University of Waterloo, 1980.

GIANOLA, D. \& FOULLEY, J.L. Variance estimation from integrated likelihood (VEIL). Génetique, Sélection, Évolution, Paris, 22:403-17, 1990.

GODOI, C.R.M. Um algorítmo eficiente para simulação de vetores com distribuição multinormal. Ciência e Cultura, São Paulo, 30 (6): 701-5, 1978a.

GODOI, C.R.M. Sobre a simulação de variáveis binormais. Ciência e Cultura, São Paulo, 30 (6): 697-701, 1978b.

GOODNIGHT, J.H. \& SPEED, F.M. Computing expected mean squares. Biometrics, Washington, 36: 123-5, Mar. 1980.

GRASER, H.U.; SMITH, S.P.; TIER, B.A. Derivative-free approach for estimating variance components in animal models by restricted maximum likelihood. Journal of Animal Science, Menasha, 64: 1362-70, 1987.

GROENEVELD, E. \& KOVAK, M. A note on multiple solutions in multivariate restricted maximum likelihood covariance component estimation. Journal of Dairy Science, Champaign, 73: 2221, 1990.

HARTLEY, H.O. \& RAO, J.N.K. Maximum-likelihood estimation for the mixed analysis of variance model. Biometrika, London, 54 (1): 93-108, 1967.

HARVILLE, D.A. Maximum likelihood approaches to variance component estimation and to related problems. Journal of the American Statistical Association, Chicago, 72 (358): 320-39, 1977.

HENDERSON, C.R. Estimation of variance and covariance components. Biometrics, Washington, 9: 226-52, June 1953.

HENDERSON, C.R. Sire evaluation and genetic trends. In : ANIMAL BREEDING AND GENETICS SYMPOSIUM, 10., Champaign, 1973. Proceedings. Menasha, American Society of Animal Science, 1973. p. 41.

HENDERSON, C.R. Applications of linear models in animal breeding. Ontario, University of Guelph, 1984a. 462 p. 
HENDERSON, C.R. Estimation of variances and covariances under multiple trait models. Journal of Dairy Science, Champaign, 67 (7): 1581-9, 1984 b.

HENDERSON, C.R. Recent developments in variance and covariance estimation. Journal of Animal Science, Menasha, 63: 208-16, 1986.

HILL, W.G. \& THOMPSON, R. Probabilities of nonpositive definide between group or genetic covariance matrices. Biometrics, Washington, 34: 429, 1978.

HOCKING, R.R \& KUTNER, M.H. Some analytical and numerical comparison of estimators for the mixed A.O.V. model. Biometrics, Washington, 31: 10-27, 1975.

KEELE, J.W.; LONG, T.E.; JOHNSON, R.K. Comparison of methods of estimating variance components in pigs. Journal of Animal Science, Menasha, 69: 1428-34, 1991.

KINDERMAN, A.J. \& RAMAGE, J.G. Computer generation of normal random variables. Journal of American Statistics Association, Chicago, 71: 893-6, 1976.

LAIRD, N.M. \& WARE, J.H. Random effects models for longitudinal data. Biometrics, Washington, 38:963-6, 1982.

LAIRD, N.; LANGE, N.; STRAM, D. Maximum likelihood computation with repeated measures : application of EM algorithm. Journal of the American Statistical Association, Chicago, 82 (397): 97-105, 1987.

LaMOTTE, L.R. Quadratic estimation of variance components. Biometrics, Washington, 29: 311-30, June 1973.

LIN, C.Y. Application of singular value decomposition to restricted maximum likelihood estimation of variance components. Journal of Dairy Science, Champaign, 70 (12): 2680-4, 1987.

LIN, C.Y. \& LEE, A.J. Sequencial estimation of genetic and phenotypic parameters in multitrait mixed model analysis. Journal of Dairy Science, Champaign, 69 (10): 2696-703, 1986.

MACHADO, A.A. Desenvolvimento de uma biblioteca de objetos para uso em programas de estatística e de matematica. Piracicaba, 1993. 230p. (Doutorado - Escola Superior de Agricultura Luiz de Queiróz/USP).

MANDOUR, H.; NORDHEIM, E.V.; RUTLEDGE, J.J. Maximum likelihood estimation of variance components in repeated measures designs assuming autoregressive errors. Biometrics, Washington, 41: 287-94, Mar. 1985.

MEYER, K. Maximum likelihood procedures for estimating genetic parameters for later lactations in dairy cattle. Journal of Dairy Science, Champaign, 66(9): 1988-97, 1983. 
MEYER, K. Maximum likelihood estimation of variance components for a multivariate mixed model with equal design matrices. Biometrics, Washington, 41: 153-65, Mar. 1985.

MEYER, K. Between algorithms : a "Short Cut" restricted maximum likelihood procedure to estimate variance components. Journal of Dairy Science, Champaign, 69 (7): 1904-16, 1986.

MEYER, K. Programs to estimate variance components for individual animal models by restricted maximum likelihood (REML). User notes. Edinburg, University of Edinburg, 1988. 12p.

MEYER, K. Restricted maximum likelihood to estimate variance components for animal models with several random effects using a derivative-free algorithm. Génetique, Sélection, Évolution, Paris, 21: 317-40, 1989.

MEYER, K. Estimating variances and covariances for multivariate animal models by restricted maximum likelihood. Génetique, Sélection, Évolution, Paris, 23: 67, 1991.

MEYER, K. $\quad$ D $\quad$ F $\quad$ R $\quad$ E $\quad M \quad$ L - Version 2.1.09. User notes. Armidale, University of New England, 1993. 97p.

MISZTAL, I. Restricted maximum likelihood estimation of variance components in animal model using sparse matrix inversion and a supercomputer. Journal of Dairy Science, Champaign, 73 (1): 163-72, 1990.

MISZTAL, I. Examples of genetic evaluation and estimation of variance components. Urbana, University of Illinois, 1993. 26p.

MISZTAL, I. \& DA, Y. Property of trace in restricted maximum likelihood estimation of variance components. Journal of Dairy Science, Champaign, 73(12): 3583-5, 1990 .

MISZTAL, I. \& GIANOLA, D. Indirect solution of mixed model equations. Journal of Dairy Science, Champaign, 70:716-23, 1987.

MISZTAL, I.; LAWLOR, T.J.; SHORT, T.H.; VanRADEN, P.M. Multiple trait estimation of variance components of yield and type traits using an animal model. Journal of Dairy Science, Champaign, 75 (2): 544-51, 1992.

MOOD, A.M.; GRAYBILL, F.A.; BOES, D.C. Introduction to the theory of statistics. 3. ed. Singapore, McGraw-Hill, 1974. 564 p.

NELDER, J.A. \& MEAD, R. A simplex method for function minimization. Computer Journal, Oxford, 7: 308-13, 1965.

PATTERSON, IH.D. \& THOMPSON, R. Recovery of inter-block information when block sizes are unequal. Biometrika, London, 58: 545-54, 1971. 
PEREZ-ENCISO, M. \& MISZTAL, I. Interface for public domain sparse matrix subroutines. Urbana, University of Illinois, 1992. 11p.

POLLAK, E.J. \& QUAAS, R.L. Monte Carlo study of genetic evaluations using sequentially selected records. Journal of Animal Science, Menasha, 52: 257, 1982

POWELL, M.J.D. An efficient method for finding the minimum of a function of several variables without calculatin derivatives. Computer Journal, Oxford, 7: 155-62, 1965.

PRESS, W.H.; TEUKOLSKY, S.A.; VETTERLING, W.T.; FLANNERY, B.P Numerical recipes in fortran. 2. ed. Cambridge, University Press, 1992. 966p.

QUAAS, R.L. Computing the diagonal elements and inverse of a large numerator relationship matrix. Biometrics, Washington, 32: 949, 1976.

RAMOS, A. A. Estudo genético-quantitativo das características reprodutivas e produtivas de um plantel da raça Gir. Ribeirão Preto, 1979. 242p. (Doutorado - Faculdade de Medicina de Ribeirão Preto/USP).

RAO, C.R. Estimation of variance and covariance componentes MINQUE theory. Journal of Multivariate Analysis, San Diego 1: 257-75, 1971a.

RAO, C.R. Minimum variance quadratic unbiased Estimation of Variance Componentes. Journal of Multivariate Analisys, San Diego, 1: 445-56, $1971 \mathrm{~b}$.

RODDA, D.D.; SCHAEFFER, L.R; MULLEN, K; FRIARS, G.W. Measuring the precision of genetic parameters by a simulation technique. Theoretical Applied Genetics, Berlin, 51: 35-9, 1977.

SCHAEFFER, L.R. Estimation of variance and covariances within the allowable parameter space. Journal of Dairy Science, Champaign, 69 (1):187-94, 1986.

SCHAEFFER L.R. Variance component estimation methods. Ontario, University of Guelph, 1993. 113p. (No prelo)

SCHAEFFER, L.R.; WILSON, J.W.; THOMPSON, R. Simultaneous estimation of variance and covariance components from multitrait mixed model equations. Biometrics, Washington, 34: 199-208, Jun e 1978.

SEARLE, S.R. Topics in variance component estimation. Biometrics, Washington, 27: 1-76, Mar. 1971.

SEARLE, S.R. Notes on variance component estimation : a detailed account of maximum likelihood and kindred methodology. Ithaca, Cornell University, 1979. 145p. (Paper BU-673-M, Biometrics Unit).

SEARLE, S.R Matriz algebra useful for statistics. New York, John Wiley, 1982. $438 \mathrm{p}$. 
SMITH, S.P. \& GRASER, H.U. Estimation of variance components in a class of mixed models by restricted maximum likelihood. Journal of Dairy Science, Champaign, 69 (4): 1156-65, 1986.

SORENSEN, D.A. \& KENNEDY, B.W. Estimation of genetic variances from unselected and selected populations. Journal of Animal Science, Menasha, 59: 1213, 1984.

THOMPSON, R . The problem of negative estimates of variance-components. Annals of Mathematics Statistics, Hayward, 33: 273-5, 1962.

THOMPSON, R . Iterative estimation of variance components for non-orthogonal data. Biometrics, Washington, 25: 767-73, Dec. 1968.

THOMPSON, R. The Estimation of variance and covariance components with an application when records are subject to culling. Biometrics, Washington, 29:527-50, Sept. 1973.

TOWNSEND, E.C. \& SEARLE, S.R. Best quadratic unbiased estimation of variance components from unbalanced data in the 1-way classification. Biometrics, Washington, 27: 643-57, 1971.

VALENTE, J. Multiple trait variance-covariance component estimation procedures with missing information for some traits. A mes, 1988. 143p. (PhD - Iowa State University).

VALÉRIO FILHO, W.V. Comparação de métodos para estimação de componentes da variância através de simulação de dados. Piracicaba, 1991. 160p. (Doutorado Escola Superior de Agricultura Luiz de Queiróz/USP)

VanRADEN, P.M. Computational strategies for estimation of variance components. Ames, 1986. 112p. (PhD - Iowa State University)

VAN VLECK, L.D. The revolution in statistical computing: from least squares to DFREML. In : FORTY-FIRST ANNUAL NATIONAL BREEDERS ROUNDTABLE, St. Louis, 1992. p. 1-35.

VAN VLECK, L.D. Algorithms for simulation of animal models with multiple traits and non-additive genetic effects. Journal Series, Nebraska Agricultural Research Division, 1994. 15p. (No prelo).

VAN VLECK, L.D.\& GREGORY, K.E. Multiple-trait restricted maximum likelihood for simulated measures of ovulation rate with underlying multivariate normal distribuitions. Journal of Animal Science, Menasha, 70: 57-61, 1992.

VERNEQUE, R.S. Fatores genéticos e de meio em características produtivas e reprodutivas de um rebanho Gir leiteiro. Viçosa, 1982. 93p. (M.S. - Universidade Federal de Viçosa). 
WALTER, J.P. \& MAO, I,L. Multiple and single trait analyses for estimating genetic parameters in simulated populations under selection. Journal of Dairy Science, Champaign, 68: 91, 1985.

WEIGEL, K.A.; GIANOLA, D.; TEMPELMAN, C.A.; MATOS, C.A.; CHEN, I.H.C.; WANG, T. Improving estimates of fixed effects in a mixed linear model. Journal of Dairy Science, Champaign, 74 (9): 3174-82, 1991.

WESTLAKE, J.R. A handbook of numerical matrix inversion and solution of linear equations. New York, John Wiley, 1968. 171p. 


\section{APÊNDICE}

1. Programa em Linguagem Fortran para Simulação de Vetores com distribuição Multinormal (adaptado de VAN VLECK, 1994)

$\mathrm{C}-$

C PROGRAMA SIMULA.FOR. Adaptado de VAN Vleck (1994)

C Journal Ser., Nebraska Agric. Res. Div., Univ. of Nebraska, c Lincolns 68583-0908

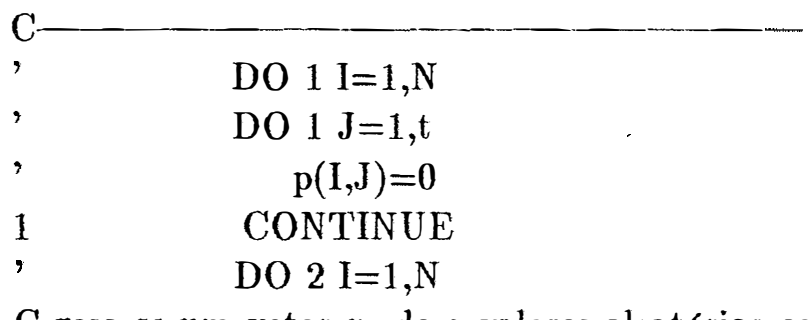

$\mathrm{C}$ gera-se um vetor $v_{g}$ de q valores aleatórios com distribuição normal

$\mathrm{C}$ gera-se um vetor $v_{e}$ de q valores aleatórios com distribuição normal C Calculo de $L_{G} v_{g} e L_{E} v_{e}$

,

,

C Acumula os produtos de $L_{G} v_{g}$ pelos elementos apropriados de $L_{A}$ ,

$\rightarrow$

C Se $\mathrm{i}=\mathrm{j}$ então adiciona os termos nas características

C Acrescenta-se também efeito aleatório adicional (não correlacionado)

$\mathrm{C}$ e que não envolve a matriz de parentesco $\mathrm{A}$, digamos $\mathrm{P}(\mathrm{K})$ para efeito 
C permanente de meio (apenas para os animais com registros)

,

6

IF (I.EQ.J $) p(J, K)=p(J, K)+E(K)$

CONTINUE

$\mathrm{C}$ Aqui podem ser acrescentados efeitos fixos para cada animal 5

2 CONTINUE CONTINUE

STOP

, END 